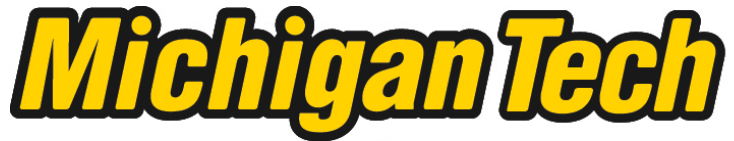 \\ Michigan Technological University Create the Future Digital Commons @ Michigan Tech
}

\section{Aerothermodynamic cycle analysis of a dual-spool, separate- exhaust turbofan engine with an interstage turbine burner}

Ka Heng Liew

Michigan Technological University

Follow this and additional works at: https://digitalcommons.mtu.edu/etds

Part of the Mechanical Engineering Commons

Copyright 2006 Ka Heng Liew

\section{Recommended Citation}

Liew, Ka Heng, "Aerothermodynamic cycle analysis of a dual-spool, separate-exhaust turbofan engine with an interstage turbine burner", Dissertation, Michigan Technological University, 2006.

https://doi.org/10.37099/mtu.dc.etds/382

Follow this and additional works at: https://digitalcommons.mtu.edu/etds

Part of the Mechanical Engineering Commons 


\title{
AEROTHERMODYNAMIC CYCLE ANALYSIS OF A DUAL-SPOOL, SEPARATE-EXHAUST TURBOFAN ENGINE WITH AN INTERSTAGE TURBINE BURNER
}

\author{
By \\ KA HENG LIEW \\ A DISSERTATION \\ Submitted in partial fulfillment of the requirements \\ for the degree of \\ DOCTOR OF PHILOSOPHY \\ (Mechanical Engineering-Engineering Mechanics) \\ MICHIGAN TECHNOLOGICAL UNIVERSITY \\ 2006
}


This dissertation, "Aerothermodynamic Cycle Analysis of a Dualspool, Separate-exhaust Turbofan Engine with an Interstage Turbine Burner", is hereby approved in partial fulfillment of the requirements for the degree of DOCTOR OF PHILOSOPHY in the field of Mechanical Engineering-Engineering Mechanics.

DEPARTMENT of Mechanical Engineering-Engineering Mechanics

Dissertation Advisor:

Dr. Song-Lin Yang

Department Chair:

Dr. William W. Predebon

Date: 


\title{
AEROTHERMODYNAMIC CYCLE ANALYSIS OF A DUAL-SPOOL, SEPARATE-EXHAUST TURBOFAN ENGINE WITH AN INTERSTAGE TURBINE BURNER
}

\author{
Ka Heng Liew \\ Department of Mechanical Engineering-Engineering Mechanics \\ Michigan Technological University
}

\begin{abstract}
This study focuses on a specific engine, i.e., a dual-spool, separate-flow turbofan engine with an Interstage Turbine Burner (ITB). This conventional turbofan engine has been modified to include a secondary isobaric burner, i.e., ITB, in a transition duct between the high-pressure turbine and the low-pressure turbine. The preliminary design phase for this modified engine starts with the aerothermodynamics cycle analysis is consisting of parametric (i.e., on-design) and performance (i.e., off-design) cycle analyses.

In parametric analysis, the modified engine performance parameters are evaluated and compared with baseline engine in terms of design limitation (maximum turbine inlet temperature), flight conditions (such as flight Mach condition, ambient temperature and pressure), and design choices (such as compressor pressure ratio, fan pressure ratio, fan bypass ratio etc.). A turbine cooling model is also included to account for the effect of cooling air on engine performance. The results from the on-design analysis confirmed the advantage of using ITB, i.e., higher specific thrust with small increases in thrust specific fuel consumption, less cooling air, and less NOx production, provided that the main burner exit temperature and ITB exit temperature are properly specified. It is also important to identify the critical ITB temperature, beyond which the ITB is turned off and has no advantage at all.
\end{abstract}

With the encouraging results from parametric cycle analysis, a detailed per- 
formance cycle analysis of the identical engine is also conducted for steady-state engine performance prediction. The results from off-design cycle analysis show that the ITB engine at full throttle setting has enhanced performance over baseline engine. Furthermore, ITB engine operating at partial throttle settings will exhibit higher thrust at lower specific fuel consumption and improved thermal efficiency over the baseline engine. A mission analysis is also presented to predict the fuel consumptions in certain mission phases.

Excel macrocode, Visual Basic for Application, and Excel neuron cells are combined to facilitate Excel software to perform these cycle analyses. These user-friendly programs compute and plot the data sequentially without forcing users to open other types of post-processing programs. 


\section{Acknowledgements}

First of all, I want to give all the praise and glory to my Almighty God and my Savior Jesus Christ in the heaven. Without knowing His will on my life, I would not have joined the $\mathrm{PhD}$ program in Michigan Tech, let alone completed the dissertation. I am greatly grateful for all the difficulties and testings He put upon me for my own sake in the future.

I would like to specially thank my academic advisor, Prof. Song-Lin Yang for his immeasurable support, technical instructions and shared experience. During the five years of my PhD work (2001-2006) at Michigan Tech, he gave me absolutely free reign but continuously advised me on the academic direction I should pursue.

I would also like to give my sincere thanks to Dr. Scott L. Post, Dr. Amitabh Narain, and Dr. Franz X. Tanner (Mathematical Science Department) for serving in my defense committee members. I am grateful for their comments and suggestions as I was finishing my dissertation.

This work was supported by NASA Glenn Research Center under grant no. NAG3-2759 monitored by Dr. Paul F. Penko, Dr. Cecil J. Marek, and then Dr. Dan L. Bulzan. I am grateful for the valuable suggestions and precious friendship from Dr. Marek during the collaboration with NASA Glenn from year 2002 to 2005. During this period, I also appreciate the technical data provided by Mr. Scott Jones, an aerospace engineer from NASA Glenn. In my fifth year of the PhD program, I was also awarded the MEEM Winnikow Fellowship to continue my research in this field.

Furthermore, I am greatly indebted to my 5-year researchmate and office- 
mate, Egel Urip, for his generosity and patience in instructing me how to develop a Visual Basic code with Microsoft Excel and how to gain better understanding in other fields like KIVA code (both fluid and solid phases), Gridgen, and Tecplot. Our sincere friendship will be forever remembered no matter where we go for our career development.

A sincere thank goes to Prof. Jack D. Mattingly from Mattingly Consultant, Bothell WA. Through the collaboration with NASA Glenn, I had a direct contact with Prof. Jack D. Mattingly who offered me a great deal of technical assistance on the development of the off-design Excel code. In addition, his publications on the aircraft engine design are my first reading materials (when I was new in this particular field) and also the foundation of my dissertation work. On the other hand, thank also goes to Mr. Jeffrey R. Hebron, a lead engineer of cycle analysis from Williams International who made assessment of the on-design Excel code that Egel and I developed, and raised several suggestions to improvement that could be done to enhance the functionalities of the code.

I've owed so much to Ken Heng See, who has been my best friend since $8^{\text {th }}$ grade (year 1993). After high school, we came to Michigan Tech for the $\mathrm{BS}$ degree (his major is Chemical Engineering) and later joined $\mathrm{PhD}$ program together. Throughout the years, I greatly appreciate the qualities of true friendship between us that God gave us. Another great friend of mine (since 1997) is Foo Chern Ting. Since we had the same advisor, we studied and did few research together in the same office for almost five years. Throughout the years, he never hesitated to point out my weaknesses, whether in critical thinking or my characters.

Let me also say 'thank you' to my pastor and brothers/sisters in Christ: 〈(1) Houghton Chinese Christ Disciple Church〉 Rev. Hsueh and his family (Minnetonka MN), Wenge Wei and his wife Qiong Zhang (with their children 
Paul and Esther), Ke Li and his wife Chunhua Zhao (left Houghton and currently in Arizona State U), Jiesheng Wang, Keong Aik Lau, Tian Wei Ng, Daw Don Cheam, Yifei Li, Yu Hong Wei and his wife Lin Pan (with their lovely daughter Yue), Cho Hui Lim, Shaochuan Liu, Tin Tin Yu etc; 〈(2) Others〉 Michelle Kuan (Los Angeles, CA). Through their testimonies in Christ, my faith in God is further strengthened and my life in Michigan Tech has been wonderful and meaningful!

Last, but certainly not least, I thank my family and a friend in Malaysia: (1) my parents, Ah Har Liew (currently in Taiwan) and Yoke Ying Ma, for bringing up me with their unconditional love and support, and giving me the best possible education in my youthhood so that I could be successful in my higher education in USA; (2) My brothers and sister, Ka Cheong, Ka Jun and Ka Yen, for patiently listening to the explanation of my research while I was home in Malaysia; (3) My best friend since $8^{\text {th }}$ grade, Mei Yee $\mathrm{Ng}$, for her support in my pursuit of $\mathrm{PhD}$ degree. 


\section{Table of Contents}

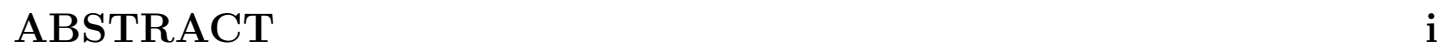

ACKNOWLEDGEMENTS iii

LIST OF FIGURES $\quad$ x

LIST OF TABLES $\quad$ Xv

NOMENCLATURE Xvi

\begin{tabular}{lll}
\hline & Introduction & $\mathbf{1}$
\end{tabular}

1.1 Background . . . . . . . . . . . . . . . . . . 1

1.1 .1 Jet Engine . . . . . . . . . . . . . . . . . . . . 2

1.1.2 Brayton Cycle . . . . . . . . . . . . . . . . . 3

1.1.3 Turbofan Engine . . . . . . . . . . . . . . . . . . . . . . . . . . . 4

1.2 Reheat Cycle . . . . . . . . . . . . . . . . . . 6

1.2.1 Interstage Turbine Burner (ITB) . . . . . . . . . . . . . 6

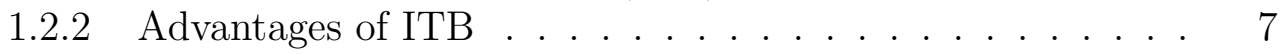

1.3 Aerothermodynamic Cycle Analysis . . . . . . . . . . . . . . . . . 9

1.4 Chapter Outline . . . . . . . . . . . . . . . . . . . . . 10

$\begin{array}{llr}2 & \text { Literature Review } & 12\end{array}$

2.1 Cycle Analyses on Aircraft Engines . . . . . . . . . . . . . . . . . 13

2.2 Cycle Analyses on Ground-based Engines . . . . . . . . . . . . . . 17

2.3 Experiments on ITB . . . . . . . . . . . . . . . . . . . . . . . 18

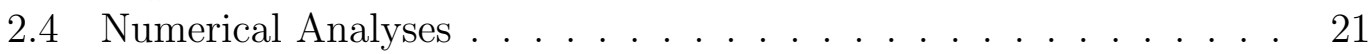

2.5 Turbine Burner and ITB Concepts . . . . . . . . . . . . . . . . . 22

2.6 Summary . . . . . . . . . . . . . . . . . . . . . . 23

2.7 Research Overview . . . . . . . . . . . . . . . . . . 23

\begin{tabular}{|l|l|}
3 & Analytical Tools
\end{tabular}

3.1 Aircraft Engine Performance Parameters . . . . . . . . . . . . . . 25

3.1.1 $\quad$ Thrust . . . . . . . . . . . . . . . . . . . . . . . . . 25

3.1 .2 Thrust Specific Fuel Consumptions . . . . . . . . . . . . . 26

3.1.3 Engine Efficiencies . . . . . . . . . . . . . . . . . . . 27

3.2 Engine Cycle and Station Numbering . . . . . . . . . . . . . . . . 28

3.3 Gas Model . . . . . . . . . . . . . . . . . . . . . . . . . . . 30 
3.4 Notations for Compressible Flow . . . . . . . . . . . . . . . . . . 30

3.5 Mass Flow Rates . . . . . . . . . . . . . . . . . . . . . . . . . . . 34

3.6 Component Efficiencies . . . . . . . . . . . . . . . . . . . . . . 36

3.6 .1 Rotating Machineries . . . . . . . . . . . . . . . . . . 36

3.6 .2 Combustion components . . . . . . . . . . . . . . . . . . 37

3.6 .3 Power transmission components . . . . . . . . . . . . . 37

4 Parametric Cycle Analysis of a Turbofan Engine with ITB 38

4.1 Introduction . . . . . . . . . . . . . . . . . . . . . . . . 38

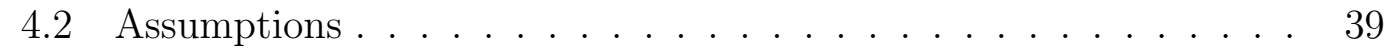

4.2 .1 Turbine Cooling . . . . . . . . . . . . . . . . . . . . . . . . . . . . . 40

4.3 Engine Component Performance . . . . . . . . . . . . . . . . . . . 41

4.3 .1 Free Stream . . . . . . . . . . . . . . . . . . . . . 41

4.3 .2 Inlet and Diffuser . . . . . . . . . . . . . . . . . . . . . . 42

4.3 .3 Low-pressure and High-pressure Compressors . . . . . . . 43

4.3 .4 Main burner and ITB. . . . . . . . . . . . . . . . . . . 44

4.3 .5 Turbine Coolant Mixer . . . . . . . . . . . . . . . . . . . 46

4.3 .6 High-pressure Turbine . . . . . . . . . . . . . . . . . . . . . . . . . . . . . 47

4.3 .7 Low-pressure Turbine . . . . . . . . . . . . . . . . . . . . . 48

$4.3 .8 \quad$ Exhaust Nozzles . . . . . . . . . . . . . . . . . . . . . . . . 50

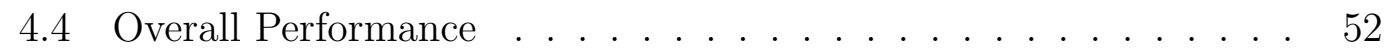

$4.4 .1 \quad$ Fan Bypass Stream . . . . . . . . . . . . . . . . . . . 52

$4.4 .2 \quad$ Engine Core Stream . . . . . . . . . . . . . . . . . . . . 53

4.5 Computer Code . . . . . . . . . . . . . . . . . . . . . . . . . . . . . . . . . . . . . . 55

4.5 .1 Code Validation . . . . . . . . . . . . . . 56

5 Performance Cycle Analysis of a Turbofan Engine with ITB 58

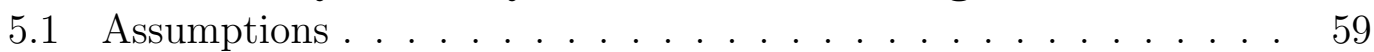

5.2 Modeling Approaches . . . . . . . . . . . . . . . . . . . 60

5.2 .1 Referencing . . . . . . . . . . . . . . . . 61

5.2 .2 Mass Flow Parameter. . . . . . . . . . . . . . . . . . . . 61

5.3 Modeling of Engine Components . . . . . . . . . . . . . . . . 62

5.3 .1 Turbine . . . . . . . . . . . . . . . . . . . 62

5.3 .2 Fan Bypass Ratio . . . . . . . . . . . . . . . . . . . 67

5.3 .3 Fan and Low-pressure Compressor . . . . . . . . . . . . . 68

5.3 .4 High-pressure Compressor . . . . . . . . . . . . . . . . . . 70

5.3 .5 Exhaust Nozzle . . . . . . . . . . . . . . . . . . . . 70

5.3 .6 Engine Mass Flow Rate . . . . . . . . . . . . . . . . . . . 71

5.3 .7 Combustor and ITB . . . . . . . . . . . . . . . . . . . . 71

5.4 Computer Code . . . . . . . . . . . . . . . . . . . . . . . . . . . . . . . . . . . 71

5.4 .1 Code Validation . . . . . . . . . . . . . . . . . . . 71

$\begin{array}{lll}6 & \text { Results and Discussions } & \mathbf{7 5}\end{array}$

6.1 Results from On-design Cycle analysis . . . . . . . . . . . . . . . 75

6.1 .1 Effect of Turbine Cooling. . . . . . . . . . . . . . 76 
$6.1 .2 \quad$ Effect of Flight Mach Number . . . . . . . . . . . . . . . . 76

6.1 .3 Effect of HPT Inlet Temperature . . . . . . . . . . . . . . 84

6.1 .4 Effect of Compressor Pressure Ratio . . . . . . . . . . . . 89

6.1 .5 Effect of Fan Pressure Ratio . . . . . . . . . . . . . . . . . . . . . . . . . . . 107

6.1 .6 Effect of Fan Bypass Ratio . . . . . . . . . . . . . . . . . . 103

6.1.7 Advantages of Operation of Main Burner and ITB Together 108

6.2 Results from Off-design Cycle Analysis . . . . . . . . . . . . . . . 112

6.2 .1 Full Throttle Performance . . . . . . . . . . . . . . . . . . 113

6.2 .2 Partial Throttle Performance . . . . . . . . . . . . . . . . 119

6.2 .3 Mission Analysis . . . . . . . . . . . . . . . . . 120

7 Conclusions and Recommendations 124

7.1 Conclusions . . . . . . . . . . . . . . . . . . . . . . . . . . 124

7.1 .1 Parametric Cycle Analysis . . . . . . . . . . . . . . . . . . 124

7.1 .2 Performance Cycle Analysis . . . . . . . . . . . . . . . . . 126

7.2 Recommendations . . . . . . . . . . . . . . . . . 127

$7.2 .1 \quad$ Increased Flexibility of the Program . . . . . . . . . . . . 128

$7.2 .2 \quad$ Multi-stage Turbine and Cooling Model. . . . . . . . . . . . 128

$7.2 .3 \quad$ Design Choice Optimization . . . . . . . . . . . . . . . . . 129

7.2.4 Separate Modeling of Fan Hub and Tip . . . . . . . . . . . 129

\begin{tabular}{ll}
\hline APPENDICES & 130
\end{tabular}

\begin{tabular}{|lr}
\hline A Summary of Papers & 131
\end{tabular}

B U.S. Standard Atmosphere 1976 134

B.1 Introduction . . . . . . . . . . . . . . . . . . . . 134

B.2 Temperature . . . . . . . . . . . . . . . . . . . . . . . 134

B.3 Pressure . . . . . . . . . . . . . . . . . . . . . . . . . 135

\begin{tabular}{ll}
\hline C Combustion Fuel Burn Model & 137
\end{tabular}

C.1 Combustion Fuel Burn Models . . . . . . . . . . . . . . . . . . 137

C.2 ENTHALPY Subroutine . . . . . . . . . . . . . . . . . 138

C.3 Products of Equilibrium Combustion . . . . . . . . . . . . . 140

C.3.1 Olikara and Borman model . . . . . . . . . . . . . . . 140

C.3.2 Adaptation to Gas Turbine Combustor and its limitations 141

C.4 NOx model . . . . . . . . . . . . . . . . . . . . . . . . . . 142

C.4.1 Oxides of Nitrogen mechanisms . . . . . . . . . . . . . 142

C.4.2 Extended Zeldovich Mechanism . . . . . . . . . . . . . . 142

C.4.3 Assumptions and Limitations . . . . . . . . . . . . . . . 143

C.4.4 Solution Procedure . . . . . . . . . . . . . . . . . . . . . 144

C.4.5 Comparison to Empirical NOx Correlations . . . . . . . . 145

\begin{tabular}{ll}
\hline D Summary of Equations for Parametric Cycle Analysis & 147
\end{tabular}

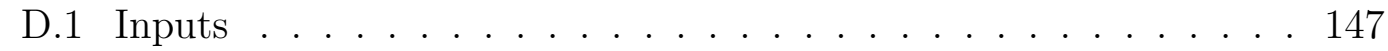

D.2 Outputs . . . . . . . . . . . . . . . . . . . . 148 
D.3 Equations . . . . . . . . . . . . . . . . . . . . . . . . . . . 148

E Summary of Equations for Performance Cycle Analysis 153

E.1 Inputs . . . . . . . . . . . . . . . . . . . . . 153

E.2 Outputs . . . . . . . . . . . . . . . . . . . . 154

E.3 Equations . . . . . . . . . . . . . . . . . . . . . . . . 154

\begin{tabular}{|lll}
\hline F Engine Control Limit System & 163
\end{tabular}

F.1 Introduction . . . . . . . . . . . . . . . . . 163

F.2 Variables of Engine Control System . . . . . . . . . . . . . . 164

F.2.1 Dimensionaless Freestream Total Temperature . . . . . . . 164

F.2.2 $\quad$ Theta Break . . . . . . . . . . . . . . . . . . . . . . . 165

F.3 Types of Control Limits _. . . . . . . . . . . . . . . . . . . . 165

F.3.1 Material Limitation . . . . . . . . . . . . . . . . . . 165

F.3.2 Compressor Pressure Ratio. . . . . . . . . . . . . . . . . 165

F.3.3 $\quad$ Engine Speed $\ldots \ldots \ldots \ldots$. . . . . . . . . . . . . . . 165

F.4 Relationship between $T_{t 4}, T_{t 4.5}$ and $\pi_{c} \ldots \ldots \ldots \ldots$

F.4.1 High-Pressure Compressor . . . . . . . . . . . . . . . 167

F.4.2 Low-Pressure Compressor . . . . . . . . . . . . . . . 168

F.5 Limiting Procedures $\ldots \ldots \ldots \ldots$

F.5.1 Reference Conditions . . . . . . . . . . . . . . 170

F.5.2 Limiting $T_{t 4}$ and $T_{t 4.5} \ldots \ldots \ldots \ldots \ldots \ldots$

F.5.3 Limiting $\pi_{f}$ and $\pi_{c H} \ldots \ldots \ldots \ldots \ldots \ldots \ldots$

F.5.4 Numerical Method for Reducing Temperature . . . . . . . 173

G User Guide to the Parametric Cycle Analysis Code 175

G.1 Definitions . . . . . . . . . . . . . . . . . . . . . 175

G.2 $\quad$ Program outline. . . . . . . . . . . . . . . . . . . . . . . . . 176

G.2.1 'CoverPage' sheet . . . . . . . . . . . . . . . . . 176

G.2.2 'Instructions' sheet . . . . . . . . . . . . . . . . . . 176

G.2.3 'Input'sheet . . . . . . . . . . . . . . . . . . . . 177

G.2.4 Plot sheets. . . . . . . . . . . . . . . . . . . . . . . . . . . . . 179

G.2.5 Data sheets . . . . . . . . . . . . . . . . . . . . . . . . . . 179

G.2.6 'Singlept' sheet . . . . . . . . . . . . . . . . . . . . . 179

G.2.7 'Station' sheet . . . . . . . . . . . . . . . . . . . . . . . . 179

G.3 Multiple-point calculations . . . . . . . . . . . . . . . . . . . . . . 179

G.3.1 "Vs Compressor Pressure Ratio" . . . . . . . . . . . . . 179

G.3.2 "Vs Flight Condition Mach Number"] . . . . . . . . . . . . 182

G.3.3 "Vs Fan Pressure Ratio" . . . . . . . . . . . . . . . . . . . . 183

G.3.4 "Vs ByPass Ratio" . . . . . . . . . . . . . . . . . . . . 183

G.3.5 "Vs Main Burner Exit Temperature" . . . . . . . . . . . . 184

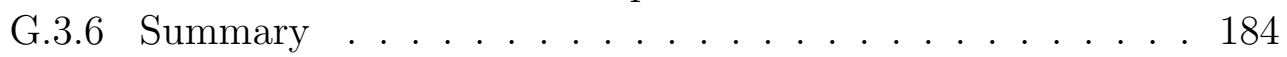

G.4 Single Point Calculation . . . . . . . . . . . . . . . . . . 186

G.4.1 Exporting Reference Engine Data . . . . . . . . . . . . 187

G.5 Engine Station Data . . . . . . . . . . . . . . . . . . . . . . . . . 189 
G.6 Discussions . . . . . . . . . . . . . . . . . . . . . . . . . . . . . 192

G.7 Troubleshooting . . . . . . . . . . . . . . . . . . . . . . . . . . . 192

G.7.1 Out of Range . . . . . . . . . . . . . . . . . . . . 192

G.7.2 $\quad$ Plotting errors . . . . . . . . . . . . . . . . . . 193

H User Guide to the Performance Cycle Analysis Code 195

H.1 Definitions . . . . . . . . . . . . . . . . . . . . . . . . . . . . . . 195

H.2 User Inputs . . . . . . . . . . . . . . . . . . . . . . . 196

H.2.1 'CoverPage' sheet . . . . . . . . . . . . . . . . . . . . . 196

H.2.2 'Instructions' sheet . . . . . . . . . . . . . . . . . . . . . 196

H.2.3 'Input' sheet . . . . . . . . . . . . . . . . . . . . . . . . 196

H.3 Full Throttle Performance over M0 and Altitude (FTP) . . . . . . 199

H.3.1 Multiple-point Calculations ('Control_FTP'sheet)] . . . . 199

H.3.2 Single-point Calculations ('SinglePt_FTP'sheet)] . . . . . 200

H.4 Partial Throttle Performance (PTP) $\ldots \ldots$. . . . . . . . . . 201

H.5 Discussions . . . . . . . . . . . . . . . . . . . . . . . . . . . . . . 203

H.5.1 Storing Data . . . . . . . . . . . . . . . . . . 203

H.5.2 Troubleshooting. . . . . . . . . . . . . . . . . 203

H.6 Summary . . . . . . . . . . . . . . . . . . . . . . . . . . . . . . 204

\begin{tabular}{ll}
\hline Flowchart of Code Structure & 206
\end{tabular}

I.1 Parametric Cycle Analysis . . . . . . . . . . . . . . . . 206

I.2 $\quad$ Performance Cycle Analysis . . . . . . . . . . . . . . . . . . 212

\begin{tabular}{|ll}
\hline$J$ & Summary of Input Data for Each Computation \\
\hline
\end{tabular}

K Other Contribution: Tracking a Single Evaporating Drop in a

Crossflow 221

K.1 Introduction . . . . . . . . . . . . . . . . . . . . . . . 221

K.2 Droplet Trajectories and Velocities . . . . . . . . . . . . . . 222

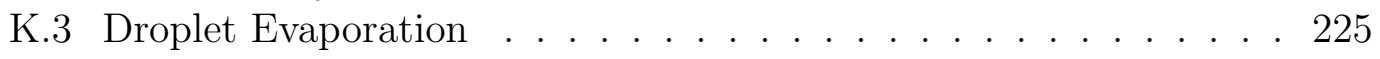

K.3.1 $\quad$ Steady-state Analysis . . . . . . . . . . . . . . . . . 225

K.3.2 $\quad$ Heat-up Process . . . . . . . . . . . . . . . . . . . . . . . . 232

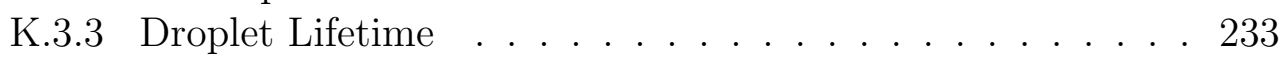

K.3.4 Convective Effect . . . . . . . . . . . . . . . . . . . . . . 234

K.4 Numerical Method . . . . . . . . . . . . . . . . . . . . . . . . . 234

\begin{tabular}{ll}
\hline BIBLIOGRAPHY & 239
\end{tabular}

VITA 244 


\section{List of Figures}

1.1 Schematic diagram of a gas generator $\ldots \ldots \ldots \ldots . \ldots . \ldots$

$1.2 \quad T$-s diagram of an ideal Brayton cycle . . . . . . . . . . . . . . 4

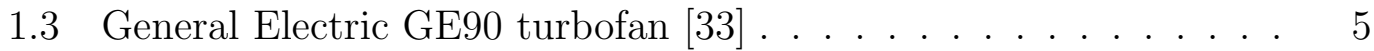

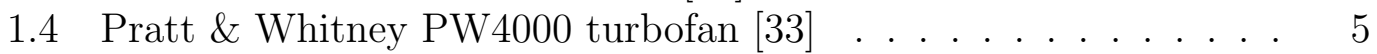

\begin{tabular}{lllll}
\hline 1.5 & $T-s$ diagram of an ideal Brayton cycle with reheat process & $\ldots$ & 6
\end{tabular}

$1.6 \quad$ (a) Increased thrust,and (b) $N O_{x}$ reduction $\ldots \ldots \ldots \ldots$

1.7 Typical gas turbine design procedure $\ldots \ldots \ldots \ldots . \ldots . \ldots 11$

2.1 T-s diagram of a jet engine with afterburning $\ldots \ldots \ldots \ldots$

$2.2 \quad T-s$ diagram of a jet engine with turbine burner . . . . . . . . . 14

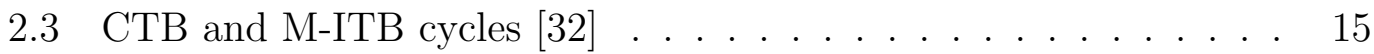

2.4 Comparison of SCC (GT26) and conventional (GT13E2) gas tur-

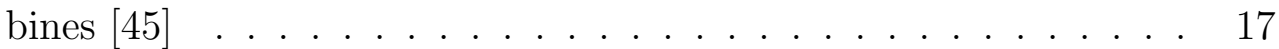

\begin{tabular}{lllllll}
2.5 & UCC showing circumferential cavity and turbine [56] & $\ldots$ & $\ldots$ & $\ldots$ & 19 \\
\hline
\end{tabular}

2.6 Cutaway comparison of a conventional gas turbine engine (bottom) and a gas turbine engine with UCC features $[56] \ldots \ldots \ldots$

3.1 Momentum fluxes and pressure forces difference on an aircraft

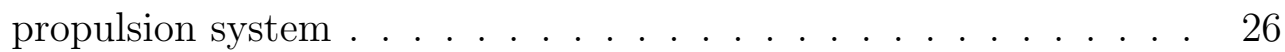

3.2 Schematic diagram of a turbofan engine with ITB $\ldots \ldots \ldots \ldots$

3.3 Engine layout with cooling air flow $\ldots \ldots \ldots \ldots$

4.1 Correlation curves of cooling air percentage (stator and rotor) vs. stator outlet temperature $\left(T_{t 4.1}\right)[54] \ldots \ldots \ldots \ldots . \ldots \ldots$

4.2 Comparison of the computed turbofan engine performance (without ITB) versus $M_{0}$ at an altitude of $10 \mathrm{~km}, \pi_{f}=2.0, \pi_{c}=28.48$, $\pi_{c L}=2.0, T_{t 4}=1500 \mathrm{~K}$, and $\alpha=4.0 . \ldots \ldots \ldots \ldots$

5.1 Variation in the HPT operating conditions with increasing $\sqrt{\tau_{i t b}} / A_{4.5} 65$

5.2 Variation in the LPT operating conditions with increasing

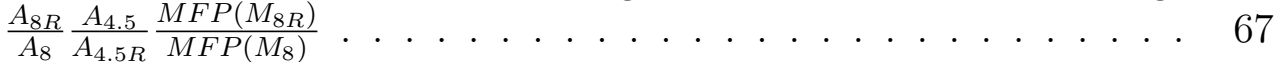

5.3 Comparison of the computed turbofan engine full throttle performance (without ITB) versus $M_{0}$ at an altitude of $10 \mathrm{~km}, M_{0 R}=$ $0.9, \pi_{f R}=2.0, \pi_{c R}=28.48, \pi_{c L R}=2.0, T_{t 4 R}=1500 \mathrm{~K}, \alpha_{R}=4.0$,

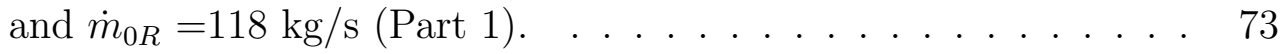


5.4 Comparison of the computed turbofan engine full throttle performance (without ITB) versus $M_{0}$ at an altitude of $10 \mathrm{~km}, M_{0 R}=$ $0.9, \pi_{f R}=2.0, \pi_{c R}=28.48, \pi_{c L R}=2.0, T_{t 4 R}=1500 \mathrm{~K}, \alpha_{R}=4.0$,

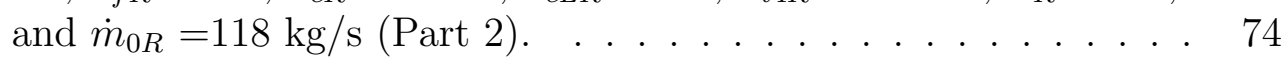

6.1 Performance comparison of turbofan engines versus $M_{0}$ at an altitude of $10 \mathrm{~km}, \pi_{f}=1.3, \pi_{c}=28.48, T_{t 4}=1800 \mathrm{~K}$, and $\alpha=4.0 .1 . \quad 78$

6.2 Performance comparison of turbofan engines versus $M_{0}$ at an altitude of $10 \mathrm{~km}, T_{t 4}=1600 \mathrm{~K}, \pi_{f}=1.3, \pi_{c}=28.48, \pi_{c L}=1.387$, and $\alpha=4.0 . \ldots \ldots \ldots$. . . . . . . . . . . . . . 81

6.3 Performance comparison of turbofan engines versus $M_{0}$ at an altitude of $10 \mathrm{~km}, T_{t 4}=1600 \mathrm{~K}, \pi_{f}=2.5, \pi_{c}=28.48, \pi_{c L}=1.387$, and $\alpha=0.5$. . . . . . . . . . . . . . . . . . . . . . . . . . . . 83

6.4 Performance comparison of turbofan engines versus $T_{t 4}$ at an altitude of $10 \mathrm{~km}, M_{0}=0.85, \pi_{f}=1.3, \pi_{c}=28.48, \pi_{c L}=1.387$, and $\alpha=4.0$. . . . . . . . . . . . . . . 86

6.5 Variation of critical ITB temperature $\left(T_{c i}\right)$ with $T_{t 4.5}$ at $M_{0}=0.85$,

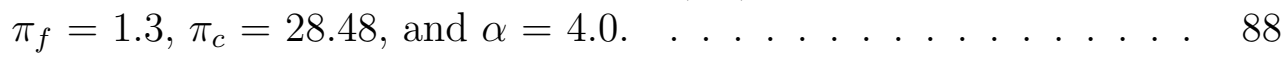

6.6 Performance comparison of turbofan engines versus $T_{t 4}$ at an altitude of $10 \mathrm{~km}, M_{0}$ of $1.2, \pi_{f}=3.0, \pi_{c}=28.48, \pi_{c L}=1.387$, and $\alpha$

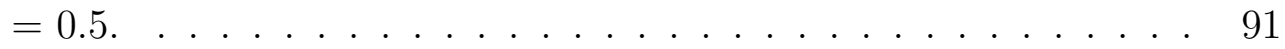

6.7 Performance comparison of turbofan engines versus compressor pressure ratio at an altitude of $10 \mathrm{~km}, M_{0}=0.85, \pi_{f}=1.3, \pi_{c L}=$ 1.387, $\alpha=0.5$, and $T_{t 4}=1600 \mathrm{~K}$. . . . . . . . . . . . . . . . . 94

6.8 Performance comparison of turbofan engines versus compressor pressure ratio at an altitude of $10 \mathrm{~km}, M_{0}=1.2, \pi_{f}=3.0, \pi_{c L}$ $=1.387, \alpha=0.5$, and $T_{t 4}=1600 \mathrm{~K}$. . . . . . . . . . . . 96

6.9 Performance comparison of turbofan engines versus fan pressure ratio at an altitude of $10 \mathrm{~km}, M_{0}=0.85, \pi_{c}=28.48, \pi_{c L}=1.387$, $\alpha=4.0$, and $T_{t 4}=1600 \mathrm{~K}$. . . . . . . . . . . . . . . . . . 100

6.10 Performance comparison of turbofan engines versus fan pressure ratio at an altitude of $10 \mathrm{~km}, M_{0}=1.2, \pi_{c}=28.48, \pi_{c L}=1.387$, $\alpha=0.5$, and $T_{t 4}=1600 \mathrm{~K}$. . . . . . . . . . . . . . . 102

6.11 Performance comparison of turbofan engines versus fan bypass ratio at an altitude of $10 \mathrm{~km}, M_{0}=0.85, \pi_{c}=28.48, \pi_{c L}=1.387, \pi_{f}$ $=1.3$, and $T_{t 4}=1600 \mathrm{~K}$. . . . . . . . . . . . . 105

6.12 Performance comparison of turbofan engines versus fan bypass ratio at an altitude of $10 \mathrm{~km}, M_{0}=1.2, \pi_{c}=28.48, \pi_{c L}=1.387, \pi_{f}$

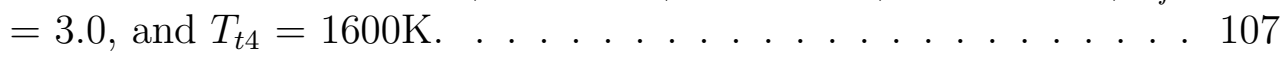

6.13 Performances of three turbofan engines versus $M_{0}, \pi_{f}=1.3, \pi_{c}=$ 28.48, and $\alpha=4.0$. . . . . . . . . . . . . . . . . . . . . . . . . 111

6.14 NOx emission of three turbofan engines versus $M_{0}, \pi_{f}=1.3, \pi_{c}=$ 28.48, and $\alpha=4.0 . \ldots \ldots \ldots 112$ 
6.15 Full-throttle performance comparison of turbofan engines (case A) versus $M_{0}$ at an altitude of $10 \mathrm{~km}, \pi_{f R}=2.43, \pi_{c R}=25, T_{t 4 R}=$ $1500 \mathrm{~K}, T_{t 4.5 R}=1300 \mathrm{~K}, \dot{m}_{0 R}=118 \mathrm{~kg} / \mathrm{s}$, and $\alpha=0.73$. . . . . . . 116

6.16 Full-throttle performance comparison of turbofan engines (case B) versus $M_{0}$ at an altitude of $10 \mathrm{~km}, \pi_{f R}=2, \pi_{c R}=28.48, T_{t 4 R}=$ $1500 \mathrm{~K}, T_{t 4.5 R}=1300 \mathrm{~K}, \dot{m}_{0 R}=550 \mathrm{~kg} / \mathrm{s}$, and $\alpha=4.0 . \quad \ldots . . .118$

6.17 Partial-throttle performance of turbofan engine (case $\mathbf{A}$ ) at an altitude of $10 \mathrm{~km}, \pi_{f R}=2.43, \pi_{c R}=25, T_{t 4 R}=1500 \mathrm{~K}, T_{t 4.5 R}=$ $1300 \mathrm{~K}, \dot{m}_{0 R}=118 \mathrm{~kg} / \mathrm{s}$, and $\alpha=0.73$. . . . . . . . . . . . . 121

6.18 Partial-throttle performance of turbofan engine (case $\mathbf{B}$ ) at altitude of $10 \mathrm{~km}, \pi_{f R}=2, \pi_{c R}=28.48, T_{t 4 R}=1500 \mathrm{~K}, T_{t 4.5 R}=1300 \mathrm{~K}$, $\dot{m}_{0 R}=550 \mathrm{~kg} / \mathrm{s}$, and $\alpha=4.0$. . . . . . . . . . . . . . . 122

C.1 Comparison of Predicted NOx Data between the simple Zeldovich model and Lefebvre's empirical model . . . . . . . . . . . . . . . . 146

F.1 CPR as a function of $\delta_{0}$ and $T_{t 4}$ for a compressor with a reference point of $\pi_{c}=\pi_{c \max }=20, T_{t 4}=T_{t 4 \max }=3300 \circ R(1833 \mathrm{~K})$, and

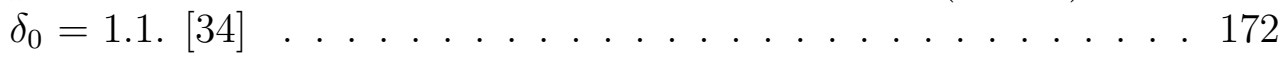

G.1 A snapshot of 'Input' sheet in on-design cycle analysis code . . . 177

G.2 A snapshot of "Vs Compressor Pressure Ratio" plot sheet in ondesign cycle analysis code . . . . . . . . . . . . . . . 180

G.3 Input parameters in "Vs Compressor Pressure Ratio" plot sheet in on-design cycle analysis code . . . . . . . . . . . . . . . 181

G.4 Tabular data for compressor pressure ratio . . . . . . . . . . . . . 181

G.5 Pull-down menu for specifying fan bypass ratios $(\alpha)$. . . . . . . . 181

G.6 On-design single point calculation sheet . . . . . . . . . . . . . . . 187

G.7 $\quad$ List of variables in the reference engine data file for use in off-design code. . . . . . . . . . . . . . . . . 188

G.8 On-design engine station data calculation sheet . . . . . . . . . . 190

G.9 Examples of temperature and pressure versus engine stations . . . 191

G.10 Pop-up windows showing warning or error messages: (a) Equations where the error occurs; (b) The current values of design variables while error is encountered. . . . . . . . . . . . . . . . 193

H.1 A view output of 'Input' sheet in off-design cycle analysis code . 198 H.2 Screenshot of Full-Throttle Performance: Multi-point calculations 200 H.3 Screenshot of Full-Throttle Performance: Single-point calculations 201 H.4 Screenshot of Partial-Throttle Performance . . . . . . . . . . . . . 202

I.1 Flow-chart of the main structure for ITB on-design Excel code. . 206

I.2 $\quad$ Flow-chart of the iterative solution scheme for on-design multiplepoint calculation. ................. . 207

I.3 Flow-chart of the iterative solution scheme for on-design singlepoint calculation. . . . . . . . . . . . . 208 
I.4 Flow-chart of the solution scheme for ITB on-design single point calculation. . . . . . . . . . . . . . . 209

I.5 Flow-chart of the solution scheme for the central ITB computation (Part 1) in on-design code. . . . . . . . . . . . . . . . . . 210

I.6 Flow-chart of the solution scheme for the central ITB computation (Part 2) in on-design code. . . . . . . . . . . . . . 211

I.7 Flow-chart of the main structure for ITB off-design Excel code. . 212

I.8 Flow-chart of the iterative solution scheme for FTP in off-design code. . . . . . . . . . . . . . . . . . . 213

I.9 Flow-chart of the iterative solution scheme for PTP in off-design

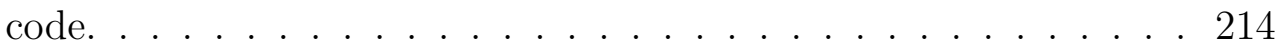

I.10 Flow-chart of the iterative solution scheme for the subroutine itbperf2 (i.e., central ITB computation) in off-design code. . . . . 215

I.11 Flow-chart of the engine control limits in off-design code. . . . . . 216

\begin{tabular}{|ll|l}
\hline K.1 & Airblast-Atomized Fuel Spray Injected Into a Crossflow of Air [23] & 222 \\
\hline
\end{tabular}

K.2 The Free-body Diagram of a Single Droplet in Crossflow [23] . . . 224

K.3 Control Surface Surronding a Single Evaporating Droplet . . . . . 227 


\section{List of Tables}

3.1 Definition for each station number . . . . . . . . . . . . . . . . . . . . . 29

3.2 Subscript definition for total property ratios (referring to station numbers in Figure 3.3$]$. . . . . . . . . . . . 33

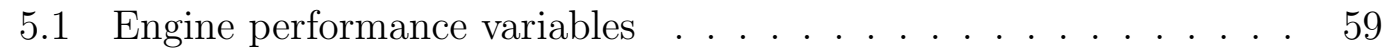

$6.1 \quad$ Design-point engine reference data . . . . . . . . . . . . . . . . . 113

6.2 Summary of results for mission analysis (24,000 lbf of take-off weight) 123

B.1 Values of several parameters at each defined levels of the earth's

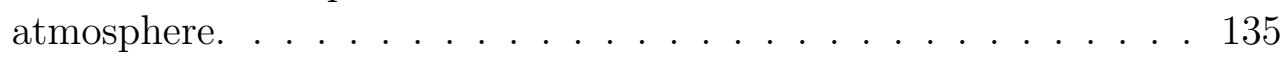

C.1 Constants for air and combustion products used in subroutine ENTHALPY $\left(\right.$ option $\left., T, f, h, C_{p}, \gamma\right) . \ldots \ldots \ldots 139$

F.1 The Logic sequence of reducing temperature (reducing fuel flow to

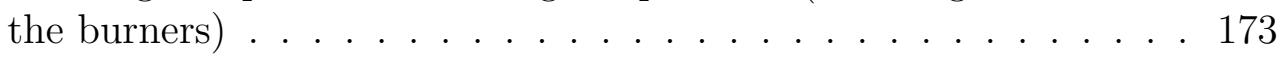

F.2 Summary of the engine control variables $Q$ and its location in the

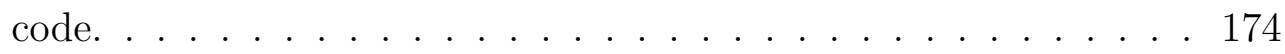

G.1 Different kinds of tools and indicators in cycle analysis codes . . . 176

H.1 List of Excel spreadsheets in off-design cycle code . . . . . . . . . 196

J.1 Turbofan engine configuration in the parametric cycle analysis. . . 217

J.2 Input data for the result comparison between the Excel codes and AEDsys suite in Chapter 4 and 5 [ . . . . . . . . . . . . . . . 218

\begin{tabular}{|ll|l|l|}
\hline J.3 Input data for the parametric cycle analysis in Chapter & (Part 1). 219 \\
\hline
\end{tabular}

\begin{tabular}{|llll}
\hline J.4 & Input data for the parametric cycle analysis in Chapter 6 (Part 2). 219 \\
\hline
\end{tabular}

\begin{tabular}{llll}
\hline J.5 Input data for the parametric cycle analysis in Chapter 6 (Part 3). 220 \\
\hline
\end{tabular}

J.6 Hidden data on 'Input' sheet of the off-design cycle analysis Excel code. . . . . . . . . . . . . . . . . 220 


\section{Nomenclature}

Note: (1) Only SI units are shown here.

(2) The asterisk $(*)$ indicates notations for the spray code.

\section{LATIN LETTERS}

\begin{tabular}{|c|c|c|}
\hline$A$ & Flow area & $m^{2}$ \\
\hline$A_{d}$ & Projected area of the droplet & $m^{2}$ \\
\hline$a$ & Sound speed & $\mathrm{m} / \mathrm{s}$ \\
\hline$C$ & Arbitrary constants & \\
\hline$c_{p}$ & Specific heat at constant pressure & $J / k g K$ \\
\hline$D$ & *Droplet diameter & $m$ \\
\hline$D_{0}$ & *Initial droplet diameter & $m$ \\
\hline$D_{F A}$ & $*$ Diffusion coefficient of fuel in air & $m$ \\
\hline 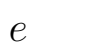 & Polytropic efficiency & \\
\hline$F$ & Uninstalled thrust or $*$ forces & $N$ \\
\hline$\frac{F}{\dot{m}}$ & Uninstalled specific thrust & $\frac{N}{k g / s}$ \\
\hline$f$ & Fuel-air ratio & \\
\hline$g_{c}$ & Newton's constant & $\mathrm{kg} \cdot \mathrm{m} / \mathrm{s}^{2}$ \\
\hline$g_{0}$ & Acceleration of gravity & $\mathrm{m} / \mathrm{s}^{2}$ \\
\hline$h$ & Altitude or enthalpy & $k m, J / k g$ \\
\hline$h_{P R}$ & Low heating value of fuel & $J / k g$ \\
\hline$h_{t}$ & Total enthalpy & $J / k g$ \\
\hline$k$ & *Thermal conductivity & $W /(m \cdot K)$ \\
\hline$k_{b}$ & Rate coefficient for backward reaction & $m^{3} / k m o l-s$ \\
\hline$k_{f}$ & Rate coefficient for forward reaction & $m^{3} / k m o l-s$ \\
\hline$L$ & *Latent heat of vaporization & $J / k g$ \\
\hline$L_{T b n}$ & *Latent heat at normal boiling point & $J / k g$ \\
\hline$M$ & *Molecular weight & $\mathrm{kg} / \mathrm{kg} \cdot \mathrm{mol}$ \\
\hline$m$ & Exponential constant & \\
\hline$\dot{m}$ & Mass flow rate or $*$ evaporation rate & $\mathrm{kg} / \mathrm{s}$ \\
\hline$\dot{m}^{\prime \prime}$ & *Evaporation rate per unit surface area & $\mathrm{kg} /\left(\mathrm{s} \cdot \mathrm{m}^{2}\right)$ \\
\hline$m_{\text {drop }}$ & *Mass of droplet & $k g$ \\
\hline$N$ & Engine speed & rpm \\
\hline 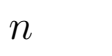 & Exponential constant & \\
\hline$n_{p}$ & Total number of mole of combustion products & kmol \\
\hline$P$ & Static pressure & $P a$ \\
\hline$P_{t}$ & Total pressure & $P a$ \\
\hline$P_{F}$ & *Partial pressure of fuel & $P a$ \\
\hline$P_{\text {vap }}$ & *Vapor pressure & $P a$ \\
\hline$\dot{Q}$ & $*$ Rate of heat release & $W$ \\
\hline$Q_{a c t}$ & *Total heat transfer to the droplet & $J$ \\
\hline$Q_{i n}$ & Rate of thermal energy available in fuel & $J$ \\
\hline
\end{tabular}




$\begin{array}{lll}Q_{s} & \text { *Steaty-state heat transfer to the droplet } & \mathrm{J} \\ R & \text { Universal gas constant } & \mathrm{J} /(\mathrm{kg} \cdot \mathrm{K}) \\ r & \text { *Radius } & \mathrm{m} \\ r_{32} & \text { *Sauter mean radius } & \mathrm{m} \\ S & \text { Uninstalled thrust specific fuel consumption } & \frac{\mathrm{mg} / \mathrm{s}}{\mathrm{N}} \\ T & \text { Static temperature or installed thrust } & \mathrm{K}, \mathrm{N} \\ T_{b n} & \text { *Normal boiling point } & \mathrm{K} \\ T_{c i} & \text { Critical ITB temperature } & \mathrm{K} \\ T_{\text {crit }} & \text { *Critical temperature } & \mathrm{K} \\ T_{d r o p} & \text { *Droplet temperature } & \mathrm{K} \\ T_{r} & \text { Reference temperature } & \mathrm{K} \\ T_{s} & \text { *Droplet surface temperature } & \mathrm{K} \\ T_{t} & \text { Total temperature } & \mathrm{K} \\ T_{\text {infty }} & \text { *Droplet ambient temperature } & \mathrm{K} \\ t_{e} & \text { *Evaporation time } & \mathrm{s} \\ T S F C & \text { Installed thrust specific fuel consumption } & \frac{\mathrm{mg} / \mathrm{s}}{\mathrm{N}} \\ U_{R} & \text { *Relative velocity between droplet and gas stream } & \mathrm{m} / \mathrm{s} \\ u & \text { *Velocity in x-direction (spray) } & \mathrm{m} / \mathrm{s} \\ V & \text { *Absolute velocity or volume } & \mathrm{m} / \mathrm{s} \text { or } \mathrm{m}^{3} \\ V_{c} & \text { Total combustion volume } & \mathrm{m} \\ w & * \text { Velocity in z-direction (spray) } & \mathrm{m} / \mathrm{s} \\ W_{\text {out }} & \text { Power } & \mathrm{W} \\ x & \text { *Horizontal direction (+ equals to the right) } & \mathrm{m} \\ Y & * \text { Mass fraction } & \\ z & \text { Geo-potential altitude } & \mathrm{km} \\ z & * \text { Vertical direction (+ equals up) } & \mathrm{m}\end{array}$

\section{GREEK SYMBOLS}

$\alpha \quad$ Fan bypass ratio

$\alpha \quad *$ Thermal diffusivity

$m^{2} / s$

$\beta \quad$ Bleed air mass fraction

$\Delta t \quad *$ time step size

$S$

$\gamma \quad$ Specific heat ratio

$\epsilon \quad$ Cooling air mass fraction

$\eta_{m H} \quad$ Mechanical efficiency for high-pressure spool

$\eta_{m L} \quad$ Mechanical efficiency for low-pressure spool

$\eta_{o} \quad$ Overall efficiency

$\eta_{p} \quad$ Propulsive efficiency

$\eta_{t h} \quad$ Thermal efficiency

$\lambda \quad *$ Evaporation constant $\mathrm{m}^{2} / \mathrm{s}$

$\mu \quad *$ Viscosity $\mathrm{m}^{2} / \mathrm{s}$

$\pi \quad$ Total pressure ratio 


$\begin{array}{lll}\rho & * \text { Density } & \mathrm{kg} / \mathrm{m}^{3} \\ \rho_{F, 288.6 K} & * \text { Density of fuel droplet at } 288.6 \mathbf{K} & \mathrm{kg} / \mathrm{m}^{3} \\ \tau & \text { Total temperature ratio } & \\ \tau_{\lambda} & \text { Total enthalpy ratio } & \\ \theta_{0} & \text { Ratio of free-stream total temperature to SLS } & \\ & \text { temperature (standard day) } & \end{array}$

\section{SUBSCRIPTS}

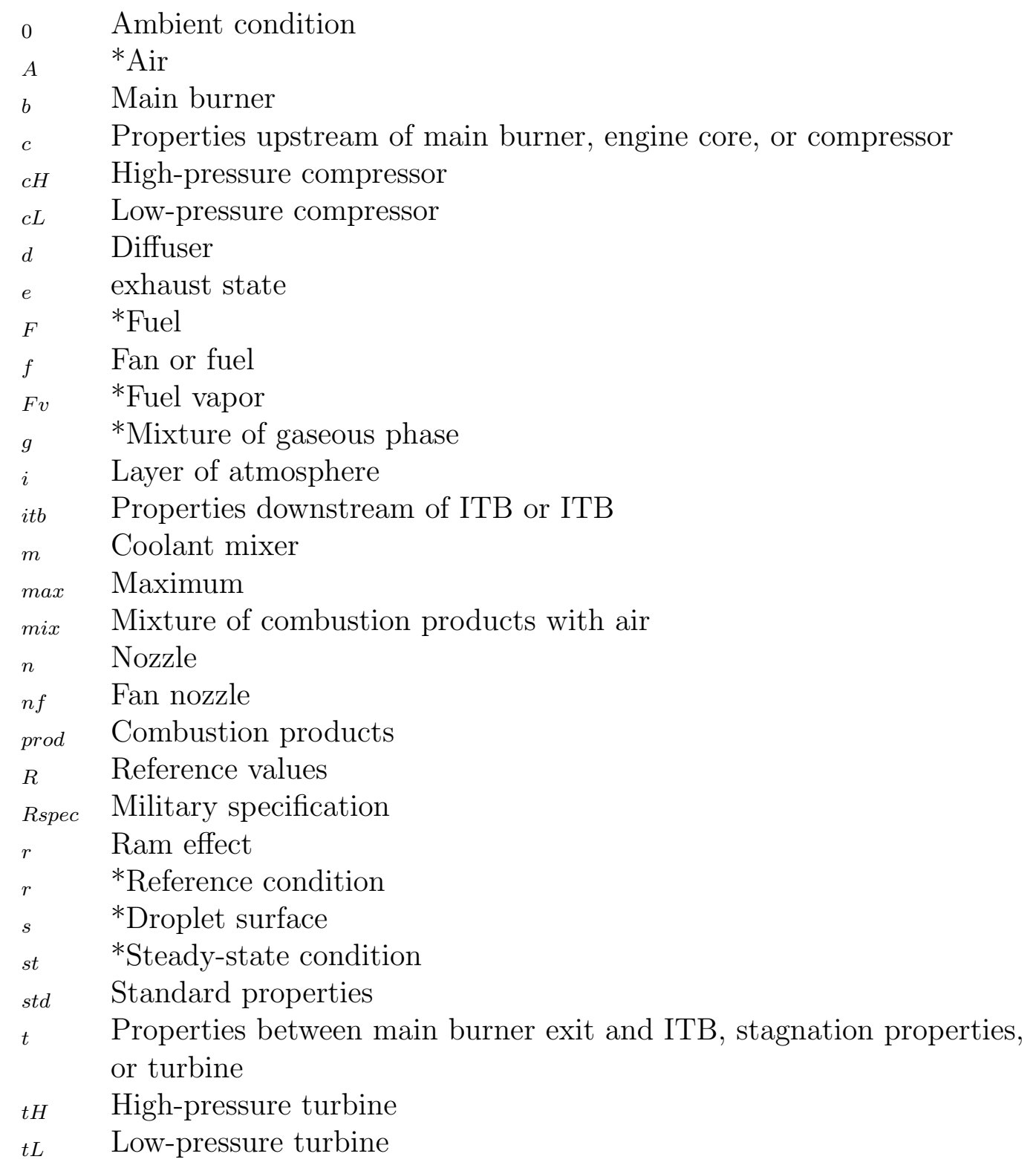




\section{DIMENSIONLESS NUMBERS}

$B_{M}$ *Mass transfer indicator of evaporation rate due to mass transfer

$B_{T} \quad *$ Heat transfer indicator of evaporation rate due to heat transfer to droplet from surrounding gas

$C_{D} \quad *$ Drag coefficient drag / dynamic force ratio

Le *Lewis drag / dynamic force ratio

$M \quad$ Mach flow / sound speed ratio

$\mathrm{Nu}$ Nusselt total / conductive heat transfer ratio

$\operatorname{Pr} \quad *$ Prandtl momentum / thermal diffusion ratio

Re *Reynolds inertia / viscous force ratio

\section{ABBREVIATIONS}

AFRL Air Force Research Laboratory

APR Aerospace Recommended Practice

CD Convergent-Divergent (nozzle)

CFD Computational Fluid Dynamics

CPR Compressor Pressure Ratio

CSH Constant Specific Heat

CTB Continuous Turbine Burner

CTT Constant Temperature Turbine

FBR Fan Bypass Ratio

FPR Fan Pressure Ratio

FTP Full Throttle Performance

HP High-pressure

HPC High-pressure Compressor

HPCPR High-pressure Compressor Pressure Ratio

HPT High-pressure Turbine

IHPTET Integrated High Performance Turbine Engine Technology

ITB High-pressure Compressor

LP Low-pressure

LPC Low-pressure Compressor

LPT High-pressure Turbine

MFP Mass Flow Parameter

MIB Multiple Interstage Burners

MSH Modified Specific Heat

NAE U.S. National Academy of Engineering

NCT Near Constant Temperature

PTP Partial Throttle Performance 
SCC Sequential Combustion Cycle

SI International System of Units

SLS Sea Level Static

ST Specific Thrust

TB Turbine Burner

TSFC Installed Thrust Specific Fuel Consumption

UCC Ultra Compact Combustor 


\section{Chapter 1}

\section{Introduction}

\subsection{Background}

Human's dream of flying over the sky was achieved with the first successful flight of the Wright brothers in December 1903. The success was then followed by a magnificent development of human-controlled, powered heavier-than-air aircraft throughout the twentieth century.

In the period from 1909 to 1940 , the aircraft was powered by the propeller/reciprocating engine propulsion system. However, this engine system reached their performance limit at the end of World War II and gradually became obsolete after the near-simultaneous invention of jet engines by Whittle in England and von Ohain in Germany in early 1940 [15].

Initially, the jet engine was developed solely for military use. Following a great deal of advancement in gas turbine technology, the first civil airplane appeared in the early 1950s. Throughout the remaining half of the $20^{t h}$ century, without any doubt, the jet engine has truly revolutionized both military and civil aviations. Until today, the gas turbine form of the jet engine is still the engine of choice for airplanes. 
In fact, U.S. National Academy of Engineering (NAE) selected Whittle and von Ohain, the independent inventors of the jet engine, as the recipients of the prestigious 1991 Charles Stark Draper Prize. The NAE citation to them read: "Their work, the turbojet engine, was completed too late to affect the outcome of the war, but it arrived in time to revolutionize the postwar world of transportation, medicine, and defense. Their work has revolutionized the world's transportation system, thus boosting the world's economy and improving the relationship between nations." [37]

In the sector of civil aviation, the worldwide boom in air travel since the past decade created an increasing market need for jumbo aircrafts, which can occupy more passengers and cargos. An engine with high propulsion power but low fuel consumption and maintenance cost is therefore highly desirable. On the other hand, the U.S. government strove to enhance military propulsion capability by initiating a national program in 1987, e.g., IHPTET (Integrated High Performance Turbine Engine Technology), which formed a coordinated effort between government, industry, and academia. These teams are producing revolutionary advancements in turbine engine technologies due to the synergistic effect of combining improved aerothermodynamics, innovative structural designs, and advanced material developments [17].

\subsubsection{Jet Engine}

The jet engine belongs to one type of gas turbines and is used to generate a high-speed jet for propulsive purposes. It accelerates and discharges a high-speed moving jet of fluid to generate thrust in accordance with Newton's Third Law of Motion. The examples of a gas turbine being used for aircraft propulsion applications include turboprops, turbojets, turbofans, and ramjets.

Generally, a gas turbine consists of an upstream compressor coupled to a downstream turbine, and a burner (also called combustor) in-between, as shown 
in Figure 1.1. These three components comprise the 'heart' of a gas turbine, namely, gas generator, in most common air-breathing propulsion engines.

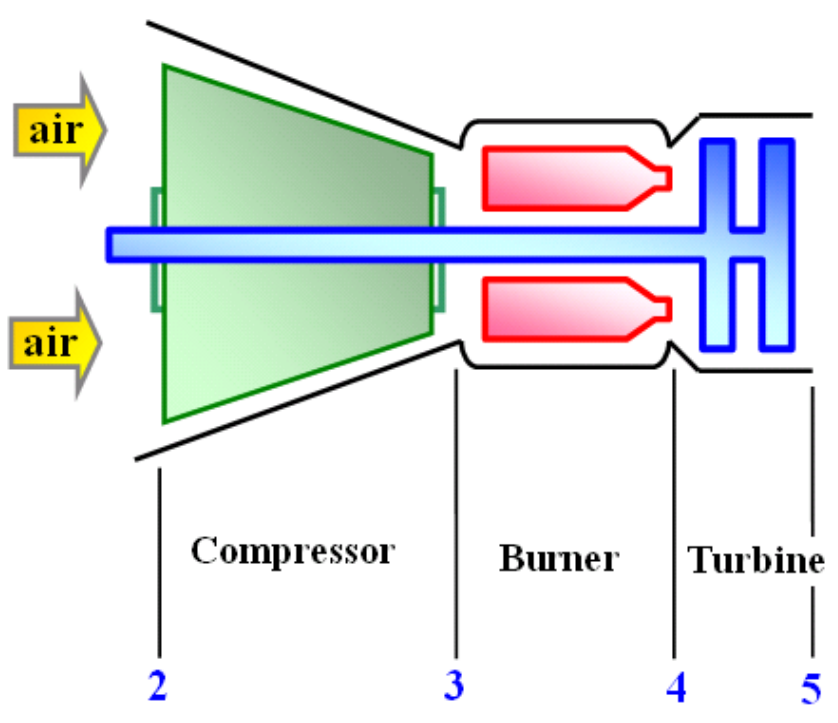

Figure 1.1: Schematic diagram of a gas generator

\subsubsection{Brayton Cycle}

The idea behind the gas generator is to convert the intake air, mixed with the fuel, into a high pressure and high temperature gas. The operation of a gas generator can be described thermodynamically by the Brayton cycle, in which the intake air is compressed isentropically (process 2-3), burned at constant pressure inside the burner (process 3-4), expanded isentropically over the turbine (process 4-5), and finally exhausted back to the starting pressure, as shown in Figure 1.2 .

Depending on the applications of the gas turbine, the energy provided is extracted and used for different applications. A turbojet engine can be constructed by adding an inlet and an exhaust nozzle to a gas generator. The exhaust nozzle converts the internal energy of the hot gas into kinetic energy or thrust. The work extracted by the turbine is to drive the compressor, or to provide auxiliary power. In addition, part of the work extracted by the turbine is also used to drive a fan for a turbofan, or a propeller for a turboprop. 


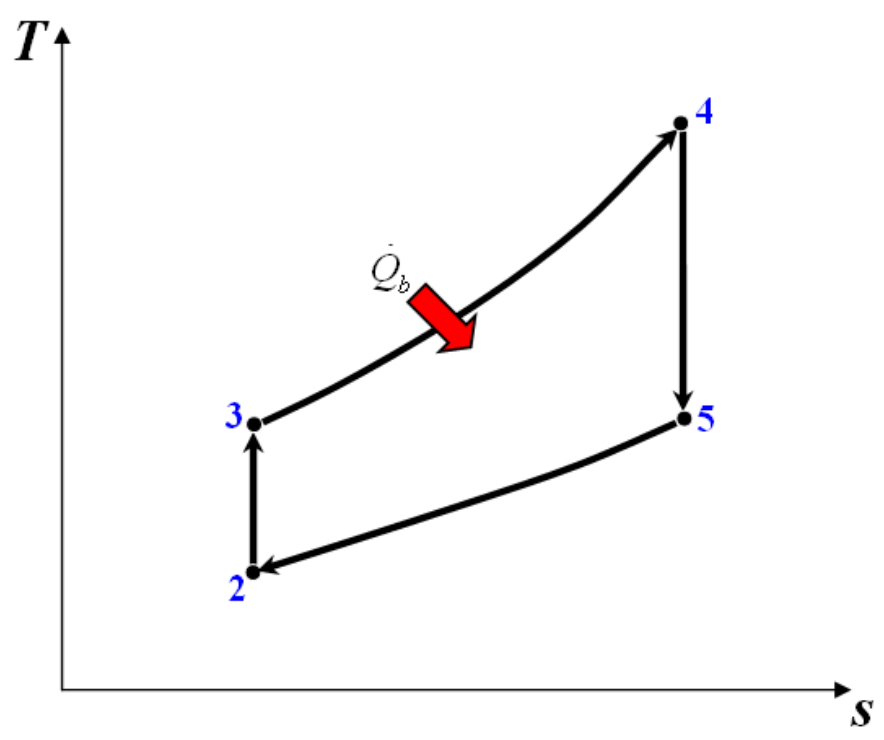

Figure 1.2: T-s diagram of an ideal Brayton cycle

\subsubsection{Turbofan Engine}

The turbofan engine, a modern variation of the basic gas turbine engine, has gained popularity in most new jet-powered aircrafts, including military and civilian types. For instances, civil-typed turbofans include General Electric GE90 (Figure 1.3), Rolls Royce Trent 500, Pratt \& Whitney PW4000 (Figure 1.4), and CFM International CFM-56 series engines. Meanwhile, military-typed turbofans include Rolls-Royce/Turbomeca Adour engines, General Electric GE F110, Pratt \& Whitney F119-PW-100, and Eurojet EJ200.

Basically, the turbofan is a turbojet engine with an addition of a fan. The fan causes more air to bypass the engine core and exit at higher speeds, resulting in greater thrust, lower specific fuel consumption and reduced noise level. Usually, the fan and low-pressure compressor are connected on the same shaft to a lowpressure turbine. A turbofan with this type of arrangement is called a two-spool turbofan engine. Since the bypass air does not mix with the engine core stream at the nozzle, the turbofan engine in this study is of the separate-exhaust type. 


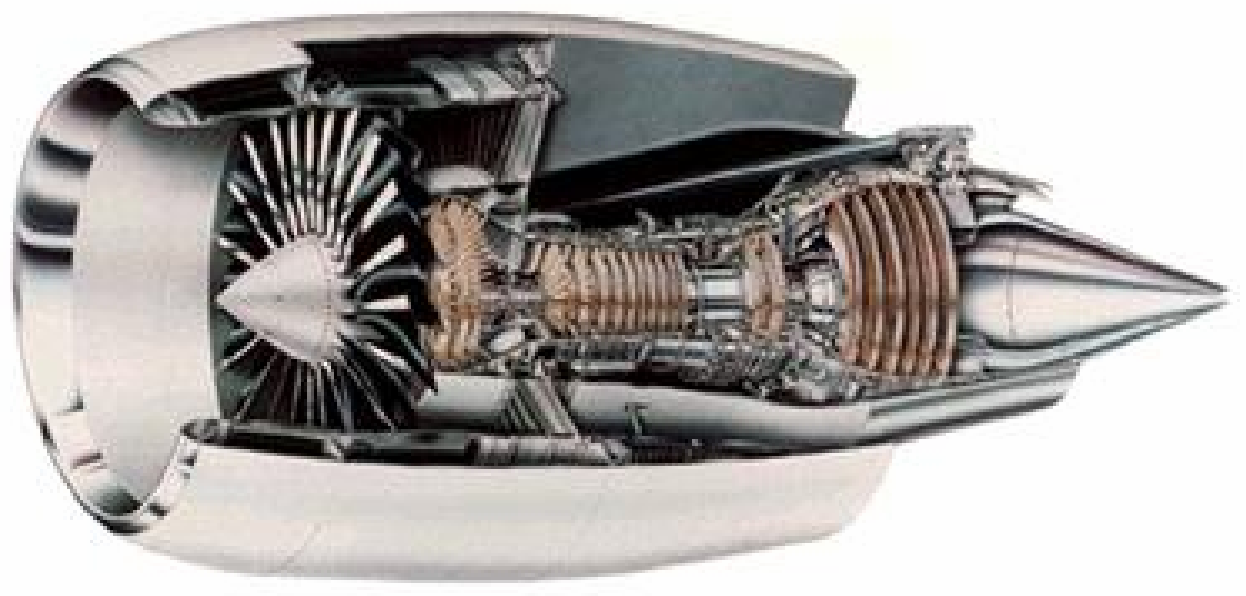

Figure 1.3: General Electric GE90 turbofan 33]

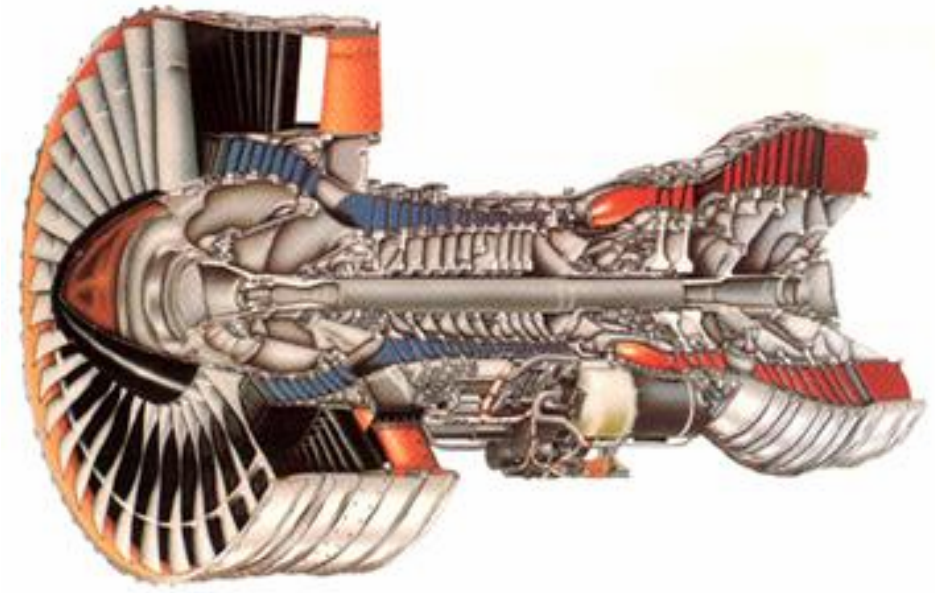

Figure 1.4: Pratt \& Whitney PW4000 turbofan [33] 


\section{$1.2 \quad$ Reheat Cycle}

The reheat cycle is an effective and widely-used method of increasing thrust quickly. It is only employed by military supersonic aircrafts, with the exception of the Concorde supersonic airliner [15].

The specific output of a Brayton cycle can be increased through a reheating process, where the expanded gas from each expansion process is reheated before the next expansion process in a turbine. The individual expansions may occur either in separate turbine machines or in different stages of a multistage machine [57]. Theoretically, one can employ an infinite number of reheat stages, leading to an isothermal expansion. Figure 1.5 illustrates an ideal Brayton cycle with a reheat process. Some research on reheat cycle that have recently been done are found in some literature and will be presented in Chapter 2

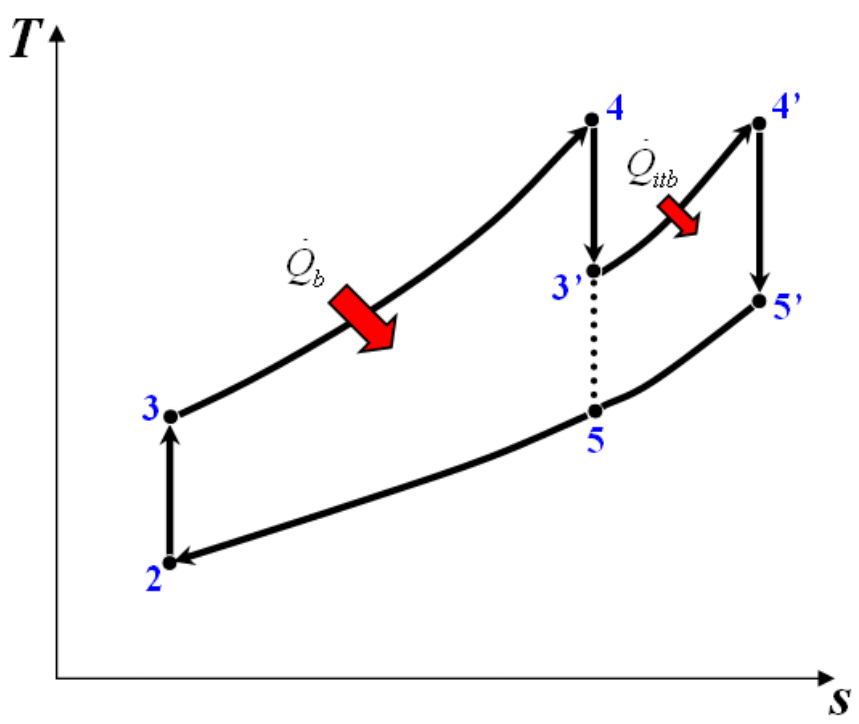

Figure 1.5: T-s diagram of an ideal Brayton cycle with reheat process

\subsubsection{Interstage Turbine Burner (ITB)}

Throughout aero-vehicle evolution, scientists and engineers have attempted to improve engine efficiency, to make it smaller, lighter, and yet more powerful. 
One of the proposed solutions to achieve these goals is the introduction of the Interstage Turbine Burner (ITB), one of the reheat cycle applications, into the engines.

Most commercial turbofan engines have a transition duct between the high-pressure turbine (HPT) and the low-pressure turbine (LPT). The ITB considered in this study is the placement of flame-holders inside the transition duct, thus becoming a secondary combustor. By doing so, only slight modification needs to be done to the existing system, without adding much size, weight, and complexity to the current engine systems. Possible modifications include a separate fuel injection system for the ITB, tougher materials for the LPT inlet guide vanes, and the associated control systems.

Referring to $T$-s diagram in Figure 1.5, the high temperature and high pressure mixture, as a result of the first heat addition process in the main burner (process 3-4), will undergo the first expansion process in the HPT (process 4-3'). It is then followed by secondary combustion in the ITB (process 3'-4'). Similar to the main burner, combustion inside ITB is ideally an isobaric or constant pressure heat addition process. The pressure loss across the ITB is estimated to have a value of between 2 to 4 percent of total pressure at ITB inlet. Lastly, the reheated high-energy mixture will undergo second expansion process across the LPT (process 4'-5').

\subsubsection{Advantages of ITB}

The major advantages associated with the use of the ITB are an increase in thrust and potential reduction in $N O_{x}$ emission, as illustrated in Figure 1.6. In Figure 1.63 , the inlet temperature of the HPT remains unchanged. As the fluid undergoes secondary combustion, a higher specific thrust is produced, as depicted in the shaded area. Figure $1.6 \mathrm{~b}$ shows the case in which the peak temperature inside the main combustor is decreased; therefore, potentially reducing the amount 
of thermal $N O_{x}$ production. Furthermore, by lowering the temperature of the main combustor, less cooling air is required for cooling the HPT blades. The loss in thrust due to a lower peak engine temperature (area A) can be compensated by secondary combustion in the ITB (area B).
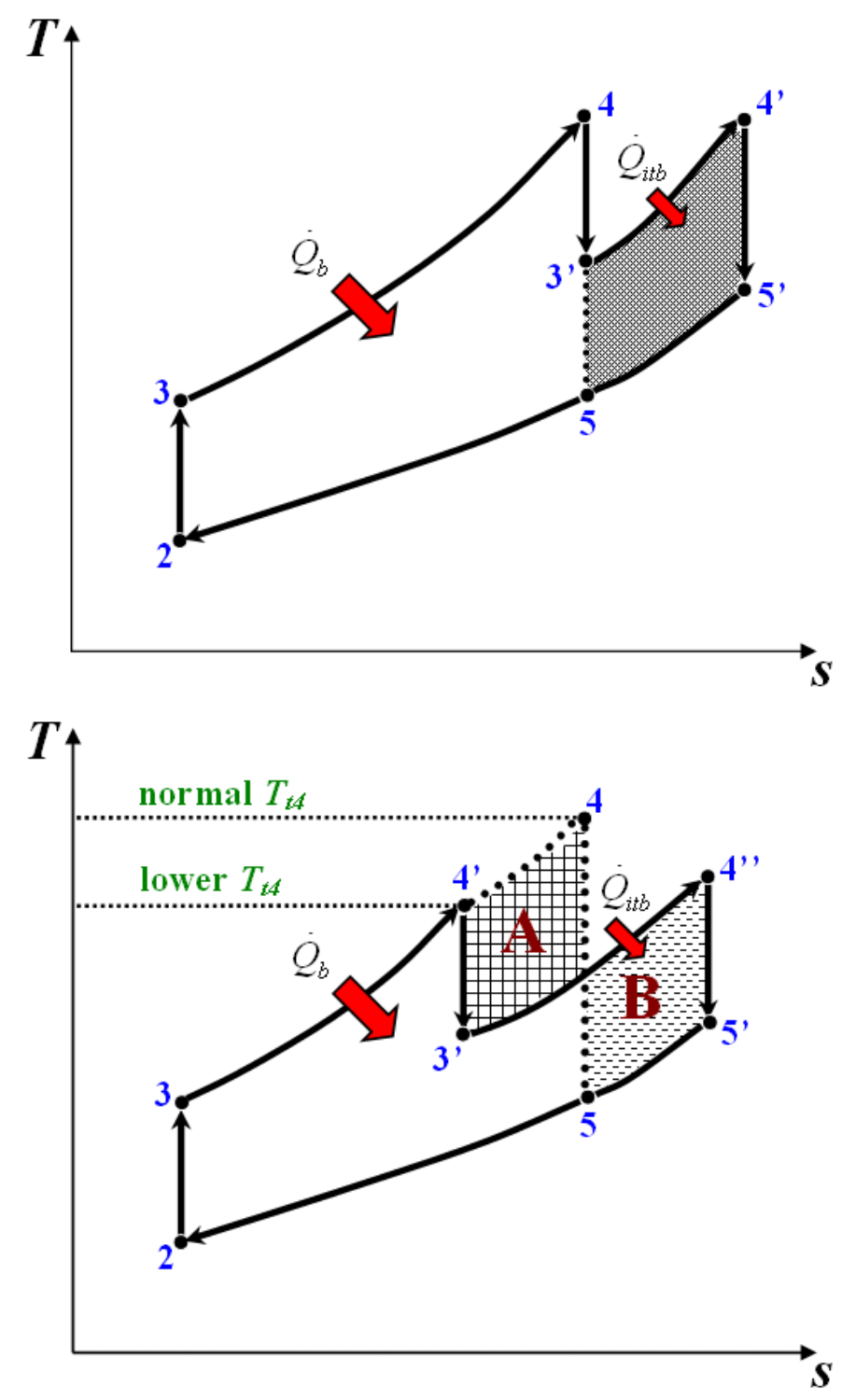

Figure 1.6: (a) Increased thrust,and (b) $N O_{x}$ reduction 


\subsection{Aerothermodynamic Cycle Analysis}

Since the introduction of the turbofan in the 1960s, much research effort is put into the development of state-of-art engine components based on the traditional turbofan cycles. However, it is a great necessity to start focusing on innovative cycles, which promise better performance and efficiency. Although the developmental cost may be higher, the improvement in performance should pay off in the long run. As a result, the investigation of the ITB cycle should start from the very beginning of a gas turbine design cycle, as shown in Figure 1.7.

Typically, the design procedure for a gas turbine always starts with the aerothermodynamic cycle analysis. It is then followed by aerodynamics study and mechanical design of turbomachinery. These three steps are often iterative until the requirements for the engine specification in mind are fulfilled. In general, aerothermodynamic cycle analysis consists of two continuous, but distinct steps, i.e., parametric and performance cycle analyses.

In order to identify the performance gain of a turbofan with an ITB, it is essential to start with parametric design cycle analysis (commonly known as on-design analysis). Then, a performance cycle analysis (also known as off-design analysis) is performed to understand how it performs at conditions other than those for which it was designed.

There are some tools available that can perform such analysis, such as Mattingly's AEDsys software suite and some cycle analysis codes developed by engine manufacturers. However, many programs with such capabilities are difficult to obtain the right to use because they are usually proprietary products, which are developed and used in-house only. A program, like the AEDsys developed by Mattingly, Heiser, and Pratt [34], is robust, user-friendly, and free to use with examples presented in their textbook ; but, this pro- 
gram is designed with specified engine configurations in mind. In addition, modifying the program will be impossible because the source code is not available.

For that reason, one of the objectives of this study is to develop two programs to perform parametric and performance cycle analyses, respectively, for the ITB engine cycle.

\subsection{Chapter Outline}

In next chapter, literature regarding the works that have been done on utilizing reheat cycle and closely-related fields is presented. The details of the parametric and performance cycle analyses are then presented in Chapter 4 and 5 , respectively. All the results associated with cycle analyses are presented and discussed in Chapter 6. The summary of the findings and recommendations for future work are presented in Chapter 77. In addition, several appendices are provided for conciseness where needed and other contribution in the gas turbine research community. 


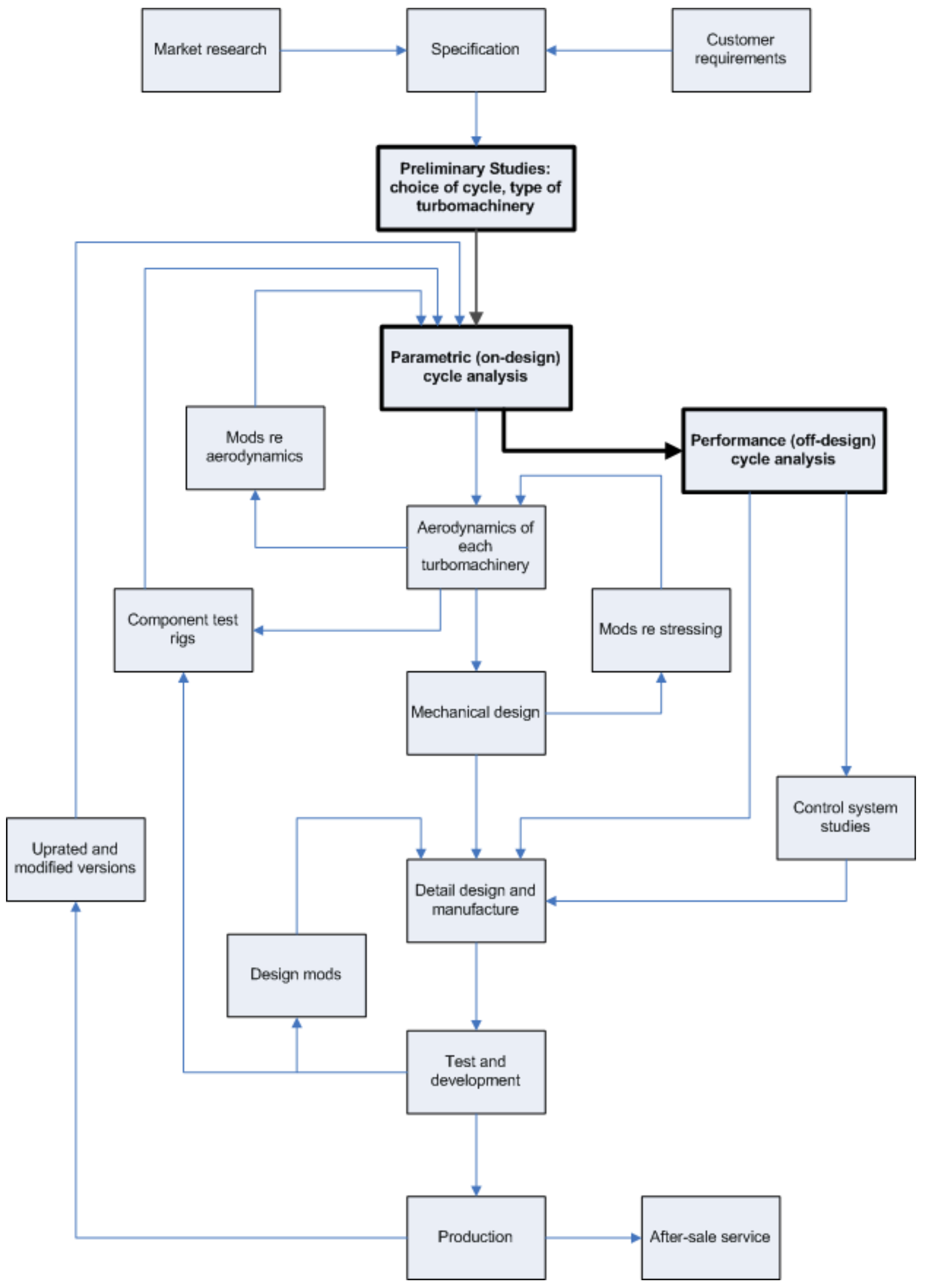

Figure 1.7: Typical gas turbine design procedure 


\section{Chapter 2}

\section{Literature Review}

In fact, the idea of reheating an expanded gas within a turbine is not new. It is now popular in power generation industry, where they utilize the reheaters (within the turbine stages) along with the intercoolers (within the compressor stages) for boosting the specific power output of the industrial gas turbines. A lot of research efforts have been put into the application of the reheat process in the ground-based power generation systems. To name a few, they are Glassman A.J. 11], Rice I.G. 42], Tekeya et al. [50, El-Masri [9], and Crane R. I. 8]. However, the application of reheaters within a turbine in aircraft gas turbine engine is rare because of its limitation of the size and weight.

Generally, the type of reheater used in an aircraft engine is called the afterburner. Afterburning, as the name implies, involves burning additional fuel and further increases the kinetic energy of the gases leaving the turbine. Typically, military jet engines utilize an afterburner for augmenting thrust. Figure 2.1 shows the $T$-s diagram for a simple jet engine with afterburning process up to $1900 \mathrm{~K}$. Due to the penalty of very high fuel consumption, an afterburner usually operates in short duration and is used only when large amount of thrust is needed for some critical mission phases. 


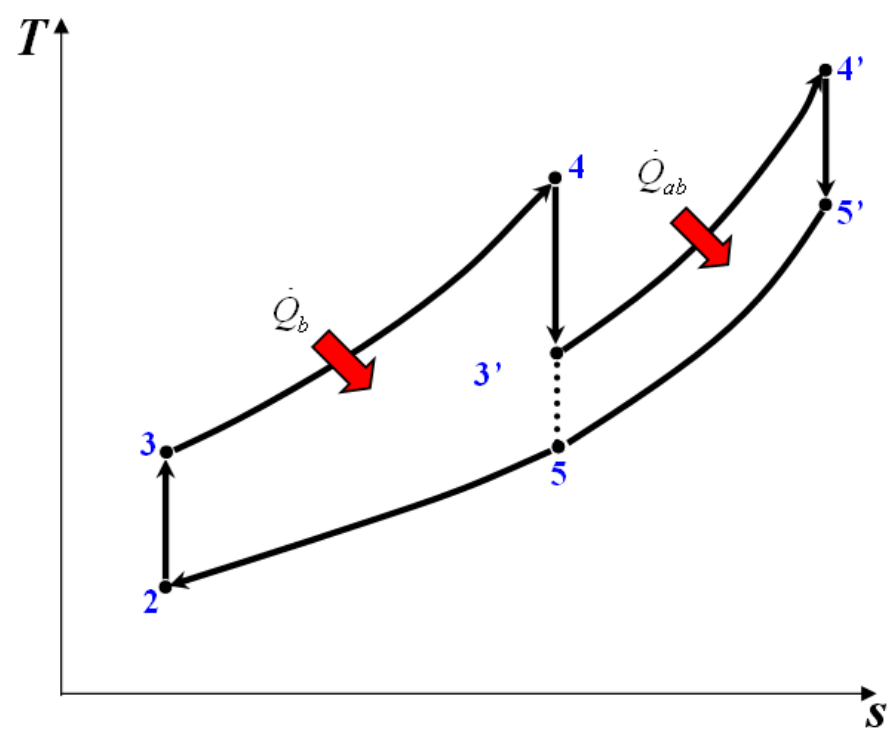

Figure 2.1: T-s diagram of a jet engine with afterburning

\subsection{Cycle Analyses on Aircraft Engines}

In an attempt to increase thrust-to-weight ratio and to widen the range of aircraft engine operation, Sirignano et al. conceived the concept of Turbine Burner (TB) in 1997 [47]. This concept was conceived to remedy the efficiency decrease due to the use of afterburner. Additional fuel was now burned in the turbine at a higher gas pressure, where the flow absorbs heat while doing work to the rotor at the same time. Ideally, it maintains constant stagnation temperature in the turbine expansion process, as shown in Figure 2.2. By doing so, the isothermal heat addition resembles those of a Carnot cycle, which is known as the most efficient cycle.

For the purpose of proof of concept, they used rather simplified assumptions, such as calorically perfect gas, constant gas properties, ideal component efficiencies, and no turbine cooling. Assuming a single-spool turbojet engine, their initial findings showed that the benefits of using TB increase with higher flight Mach number, i.e., about 20\% increase in specific thrust with only about $10 \%$ increase of thrust specific fuel consumption over a non-afterburning Brayton 


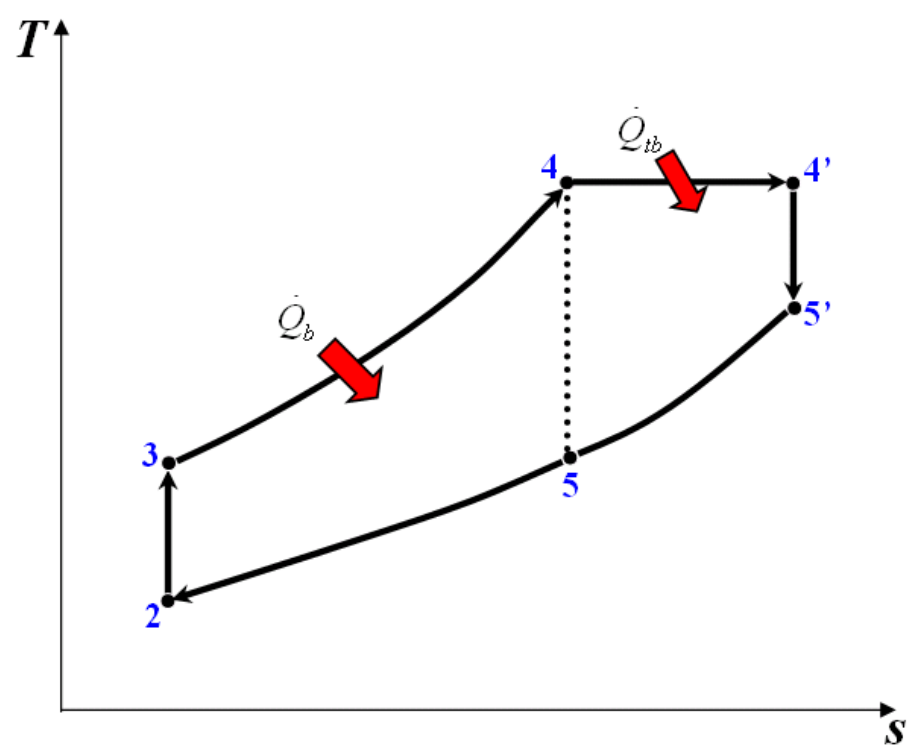

Figure 2.2: T-s diagram of a jet engine with turbine burner

cycle. Later, they found that the TB gave even greater performance gain with increasing compression ratios rather than increasing flight Mach number. At the high compression ratios, the TB engine configuration produces almost equal specific thrust as those produced by conventional afterburner configuration, without the penalty of high fuel consumption rate associated with typical afterburner design[48].

In 2001, Liu and Sirignano extended the study by introducing a variant of turbine-burner, i.e., ITB [32]. The details of component efficiencies and the method of analysis were identical to those listed in [48], except that they included a single-shaft, separate-exhaust turbofan engine.

In the ITB, the turbine stators or nozzles were converted into the combustors. Obviously, the ITB was less technically challenging compared with the $\mathrm{TB}$, in which combustion took place in the turbine rotor. To achieve isothermal heat addition, an 'infinite' number of discrete ITBs (denoted by M-ITB), each with an isentropic expansion followed by an isobaric heat addition, were used to approach a continuous turbine burner (CTB) cycle, as shown in Figure 2.3 . 
Their results showed that the engine performance increased as the number of ITBs increased.

Retaining the benefits found in their early work, they continued to present the distinct advantage of the TB engine over the conventional engine. A TB engine benefited from a larger bypass fan and a higher turbine inlet temperature. Furthermore, they emphasized the importance of finding an optimized power distribution of turbine segments in a multiple-ITB design for a better thermal efficiency.
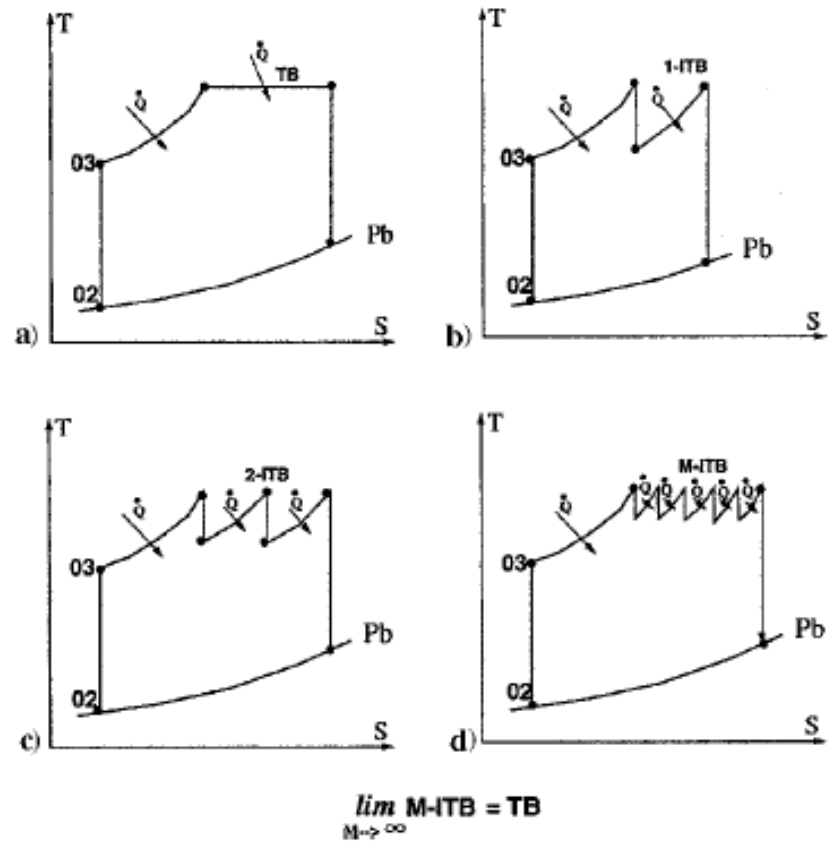

Figure 2.3: CTB and M-ITB cycles [32

Another concept similar to the ITB worth mentioning is the Sequential Combustion Cycle (SCC). Basically, SCC means that a second combustion after the HPT is used to reheat the gas before the final expansion at the LPT. In fact, ABB Power Generation has successfully designed a new family of industrial gas turbine (e.g., ABB GT26) based on the SCC concept for the power generation [45] (Figure 2.4) and has been marketing the products for some times. With this 
success, Vogeler proposed to apply the SCC concept to the high bypass ratio jet engine [53]. Vogeler asserted that the proposed SCC would allow increased thrust without pushing technology on the materials and turbine cooling system. In his analysis, the variable specific heat due to the compression, heat addition and expansion processes were taken care of by locally correcting these values from a gas table. Vogeler performed few analyses on two types of turbofan engine configurations, i.e., single-spool and twin-spool. A constant level of technology is applied in all the case studies.

According to his findings, the author emphasized the advantage of SCC on the single-spool engine over the twin-spool engine. The reason given was that the expansion of most of the high pressure and high temperature gas already took place in the HPT. The low pressure ratio across LPT did not allow an effective use of this energy in the fan. As a result, it requires that the overall engine pressure ratio must be considerably higher than the conventional engine with the same level of heat addition. It was necessary to offer enough pressure drop in the second combustor to utilize a high fuel input and transfer this energy with a good efficiency to the spool.

Andriana et al. 22 also investigated the performance gain of a turbojet engine with a so-called Constant Temperature Turbine (CTT) cycle. The CTT cycle was exactly the same as the Near Constant Temperature (NCT) cycle proposed by Liu and Sirignano [32]. Their analysis was somewhat less thorough compared with Sirignano et al.[48] and they did not compare the performance gain of CTT engine with any base engine. Nevertheless, they outlined the off-design analysis of their cycle by deriving a simple model. The model was derived by assuming that the flows at the turbine inlet and at the exhaust nozzle are choked. It yielded an expression for the compressor pressure ratio as a function of the engine inlet conditions, turbine inlet temperature, and the area ratio between the exhaust nozzle and the turbine inlet. 


\subsection{Cycle Analyses on Ground-based Engines}

With the success of the SCC concept by $\mathrm{ABB}$, it is still worthwhile to know other researchers' works of applying the ITB cycle to the land-based gas turbines. Among them include Sirignano and Liu [48] and Chen et al. [4].

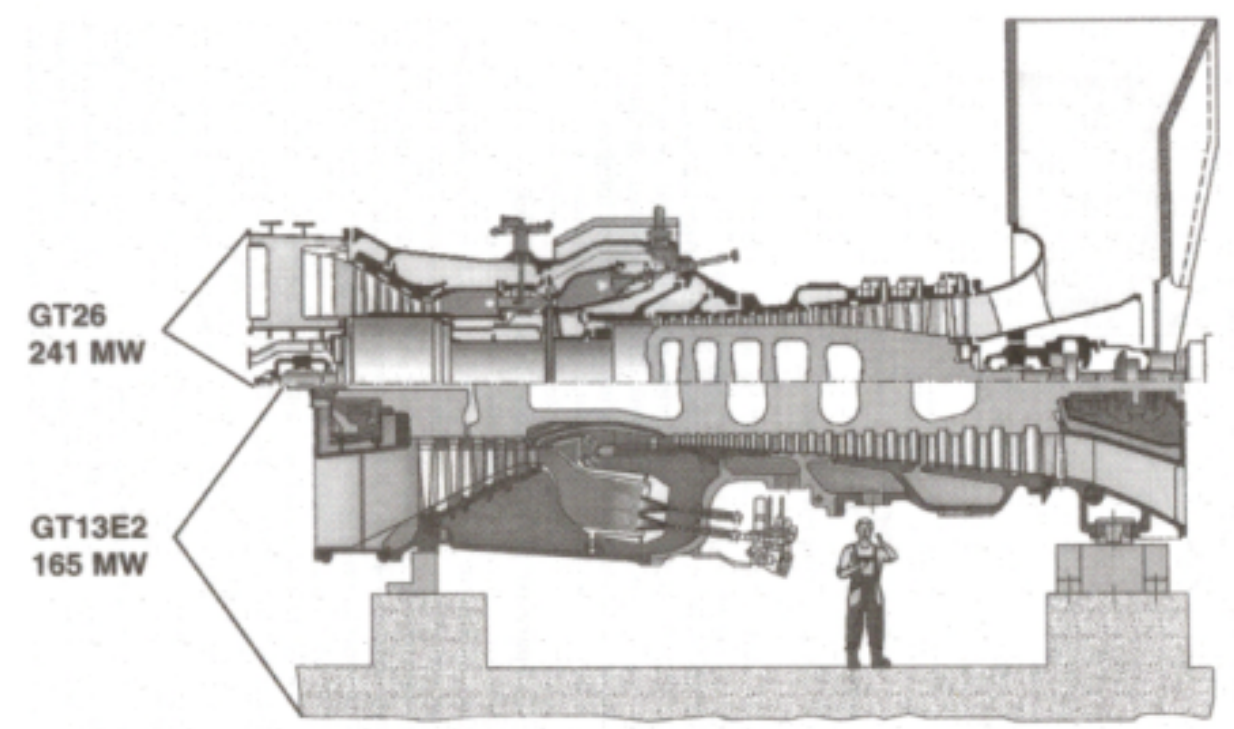

Figure 2.4: Comparison of SCC (GT26) and conventional (GT13E2) gas turbines [45]

Chen et al. investigated the engine performance with multiple interstage burners (MIB), similar to M-ITB cycle as first proposed in [32]. The authors included various turbine cooling-flow schemes and the variable specific heats into the analysis for more accurate prediction of the gas turbine engine performance. Their findings confirmed the significant improvement through the use of MIB for the land-based power production systems. Interestingly, it was found that two MIBs yield the near maximum thermal efficiency, while only one or more than two MIBs would start to deteriorate the thermal efficiency. 


\subsection{Experiments on ITB}

From the preceding reviews, much analytical effort has been put into studying the potential gain of the TB or its similar concepts. Meanwhile, some researchers at Air Force Research Laboratory (AFRL) are pilot-testing an innovative combustor, called Ultra-Compact Combustor (UCC) experimentally [56; 49]. They claimed that UCC has the potential to be used as a main burner or as an ITB that does not impact engine thrust-to-weight, pollutant emissions, or overall system performance. The UCC design integrates the compressor and turbine features, which will enable a shorter and potentially less complex gas turbine engine.

In the UCC concept, a cavity runs around the outer circumference of the extended turbine inlet guide vanes, as shown in Figure 2.5. Aligned with this cavity, within each vane, will be a radial cavity. This design allows burning rich in the circumferential cavity, and allowing much of the required combustion residence time to take place in the circumferential direction of engine, rather than the axial as is done in the conventional burners. They estimated the UCC concept would eliminate many existing engine components, leading to a $66 \%$ shorter in length than that of a conventional combustion system, as shown in Figure 2.6.

The experimental results demonstrated its advantage of having higher heat release rate by a factor of two and a shorter flame length by $50 \%$ compared to the conventional combustor designs. The success of this technology could translate to a significantly reduced engine length and weight, resulting in a higher power density machine.

The UCC design is based on the earlier experiments on combustion with high $g$-loading by Lewis [24]. In his experiments, Lewis observed that the role of centrifugal forces on enhancing the flame spreading speed to a value beyond that 
of a turbulent flame. The centrifugal forces were established up to $10^{4} \mathrm{~g}$ and the observed flame speeds were about four times that of a conventional turbulent flame speed. To explain this phenomenon, he proposed the bubble-transport theory, which stated that the "bubbles" or eddies moving ahead of the flame front due to the centripetal acceleration helped enhance turbulence, and subsequently spread the flames.

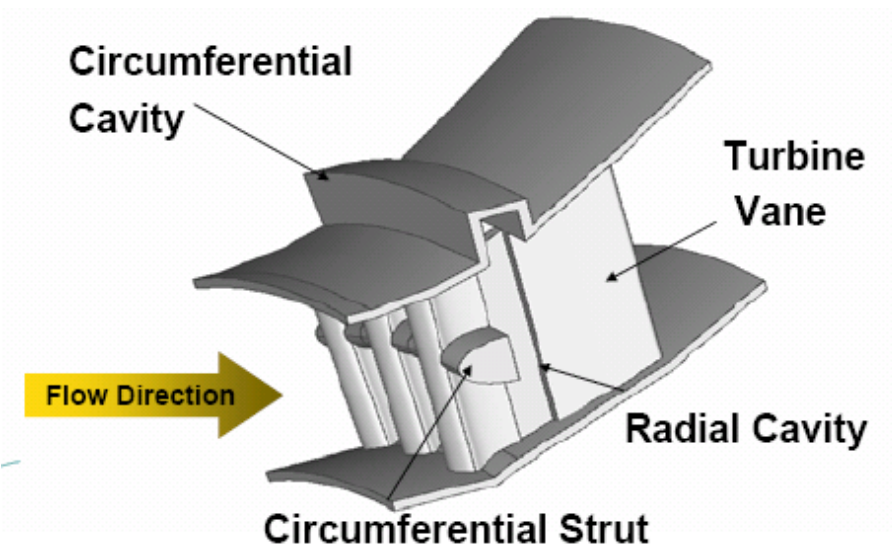

Figure 2.5: UCC showing circumferential cavity and turbine [56] 


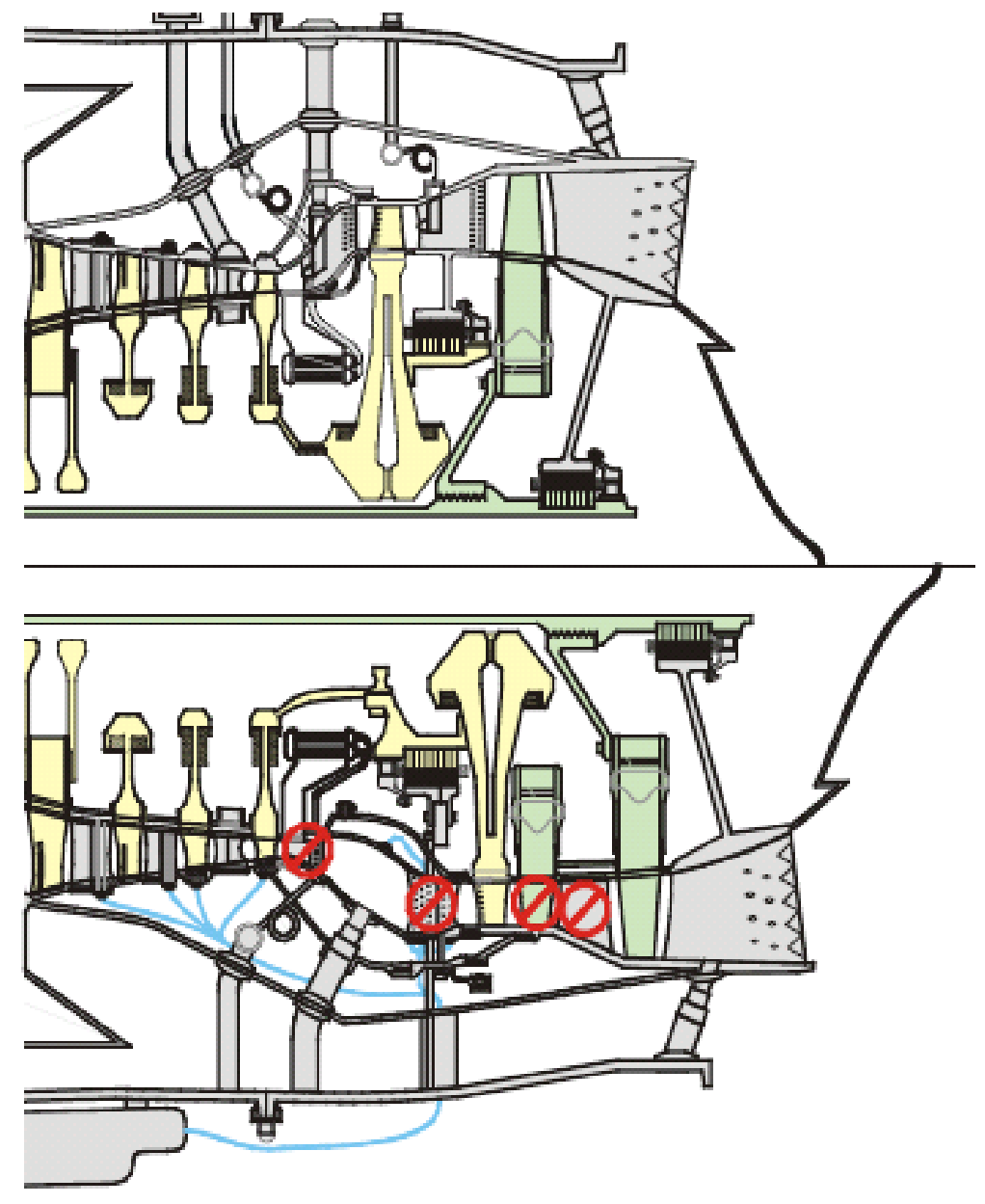

Figure 2.6: Cutaway comparison of a conventional gas turbine engine (bottom) and a gas turbine engine with UCC features [56] 


\subsection{Numerical Analyses}

For a better understanding of the phenomena of burning fuel inside a harsh environment, for instance, the turbine rotor, several researchers also performed numerical analysis on the ITB. They aimed to determine whether such combustion scheme is feasible.

Siow and Yang [46] presented the computational result of the combusting flow field inside a simplified ITB. ITB, located between LPT and HPT, was subject to different inflow velocities, flame-holder sizes and shapes. The results demonstrated that the flow and combustion stability depended strongly on Reynolds number. The author asserted that acoustic waves must be taken into consideration when designing an ITB.

Concurrent with the experimental effort at AFRL by Zelina's group [56], Mawid [35] carried out the three-dimensional computational fluid dynamics (CFD) computations to guide the ITB experiments. They claimed that it was very important to identify the key design parameters for the best performance and to optimize the ITB design configurations. The results presented that the intense combustion occurs inside the circumferential cavity (Figure 2.5). It is also noted that the radial vane cavity was very useful in transporting the hot combustion products into the circumferential cavity to mix with the main airflow.

Recently, Hendricks et al. [13] performed CFD study on a sequential twostage power generation combustion system, which was similar to GT24/GT26 industrial gas turbines. In their study, the two-stage combustor was termed as an ITB. The simulation was performed within a single computational domain, with a full coupling between the two combustors and the rotating HPT.

Computational results showed the interaction of the combustor flow and the 
HPT stages. They observed the non-uniformity of the flow as it entered the HPT flow field as well as the downstream as it entered the mixing and fueling zone of the second stage combustor. In addition, the simulation demonstrated the importance of including the rotating HPT blades in the computation, because this resulted in a direct computation of the combustion within the first turbine stage, and an accurate simulation of the flow in the second combustion stage. The direct computation of hot streaks through the rotating HPT stage led to an improved understanding of the aerodynamic relationship between the primary and secondary combustors, and the turbomachinery.

This two-stage combustor was designed to provide a stable, lean burning, and low temperature alternative to the single-stage combustors. A lower HPT inlet temperature translated directly to an improvement in the blade life durability and the reduction in pollutant emissions.

\subsection{Turbine Burner and ITB Concepts}

As one can notice above, the concept of the reheat process appears in different names in literature, such as the CTB, CTT cycle, NCT cycle, SCC, and ITB, which is quite confusing sometimes. CTB, CTT and NCT cycles are somewhat similar to TB concept, which is to maintain an isothermal combustion inside the turbine passages for a maximum thermal efficiency. On the contrary, the SCC and ITB concepts employ a separate secondary combustor after the first expansion process at HPT stage or machine. In fact, the SCC and ITB concepts mentioned here are equivalent to 1-ITB as presented in Liu and Sirignano [32] and Chen et al. [4].

Obviously, an ITB is a somewhat different concept from a TB. As stated in the preceding chapter, ITB is an isobaric or constant pressure combustor between 
two turbine stages, not inside turbine stages. Unlike the TB, the combustion in an ITB does not occur in the turbine rotors, so no work is extracted from the combusting flow. In other words, the combustion and the work extraction are decoupled in the analysis. Nevertheless, both concepts belong to the category of the reheat process and are expected to give similar trends of engine performance gain, with ITB having a less thermal efficiency and complexity.

\subsection{Summary}

In this chapter, the results from these researchers presented the worthiness of designing ITB. Nevertheless, there are several questions arising from these results. First of foremost, the turbine cooling flow is not modeled in the cycle analysis on an aircraft engine. It is found that the turbine inlet temperature used in their analysis (i.e., 1600K) is higher than the current turbine material

limit (i.e., 1370K [55]). Therefore, it is reasonable to model the turbine cooling flow in the cycle analysis. Without accounting for the cooling flow effect, any performance gain associated with ITB may be overestimated.

Most studies on the ITB are only limited to the parametric cycle analysis. Few researchers did conduct the performance analysis, but in a relatively simple and generic manners. In order to address the potential advantages and disadvantages of ITB, it is definitely a great necessity to develop an accurate performance analysis code, which is one of the important parts of this work.

\subsection{Research Overview}

As clearly seen in Figure 1.7, the present doctoral work is part of a broader effort in designing innovative gas turbine engine with a modified power cycle. 
More specifically, the present study is aimed at performing aerothermodynamic cycle analysis of a conventional turbofan engine with an ITB. Through this one-dimensional modeling and analysis, the emphasis is on accessing and revealing both its potentials and drawbacks of adding an ITB on the engine performance.

The research that have been carried out can be outlined as follows:

1. Review of the past and current research effort on the ITB or closely-related concepts to fully understand their feasibilities and limitations applicable to a typical turbofan engine.

2. A detailed approach for modeling each engine component in parametric cycle analysis, including the approximate turbine cooling model.

3. Formulation of equations for a steady-state performance cycle analysis of an ITB engine and its solution procedures.

4. Development of programs that perform a complete aerothermodynamic cycle analysis. These programs should be easy to use and widely accessible. 


\section{Chapter 3}

\section{Analytical Tools}

\subsection{Aircraft Engine Performance Parameters}

In describing the performance of a turbofan with ITB, it is helpful to define three important air-breathing engine performance parameters that are useful in aircraft propulsion. They are thrust, fuel consumption, and engine efficiencies. Each of these parameters is briefly discussed here and the detailed derivations of each term will be shown in both cycle analyses.

\subsubsection{Thrust}

Thrust is the force used to sustain a flight (thrust $=d r a g$ ), to accelerate a flight (thrust $>d$ rag), or to decelerate a flight (thrust $<$ drag). Referring to Figure 3.1, we can apply momentum balance to the control surface. Uninstalled thrust (F) of a jet engine (single inlet and single exhaust) is given by

$$
F=\frac{\left(\dot{m}_{0}+\dot{m}_{f u e l}\right) V_{e}-\dot{m}_{0} V_{0}}{g_{c}}+\left(P_{e}-P_{0}\right) A_{e}
$$

where $\dot{m}_{0}, \dot{m}_{\text {fuel }}$ are mass flow rates of air and fuel, respectively,

$V_{0}, V_{e}$ are velocities at the inlet and exit, respectively,

$P_{0}, P_{e}$ are pressures at the inlet and exit, respectively.

$g_{c}$ is the Newton's constant ( $=32.3 \mathrm{lb} / f t^{2}$ for English unit, or $=1$ for SI unit). 
$A_{e}$ is the cross-sectional area at the exit.

Ideally, the hot gas is expanded to the ambient pressure, which gives $P_{e}=P_{0}$. Equation 3.1 then becomes

$$
F=\frac{\left(\dot{m}_{0}+\dot{m}_{f u e l}\right) V_{e}-\dot{m}_{0} V_{0}}{g_{c}}
$$

The uninstalled thrust, as described above, depends on the engine alone and is independent of the nacelle. Including nacelle will give the installed thrust $(T)$, as given by

$$
T=F-D_{\text {inlet }}-D_{n o z}
$$

where $D_{\text {inlet }}$ and $D_{\text {по }}$ are the drag forces from the inlet and the nozzle, respectively.

Obviously, installed thrust $T$ is less than the uninstalled thrust $F$ because some useful forces have to be used to overcome the nacelle drags.

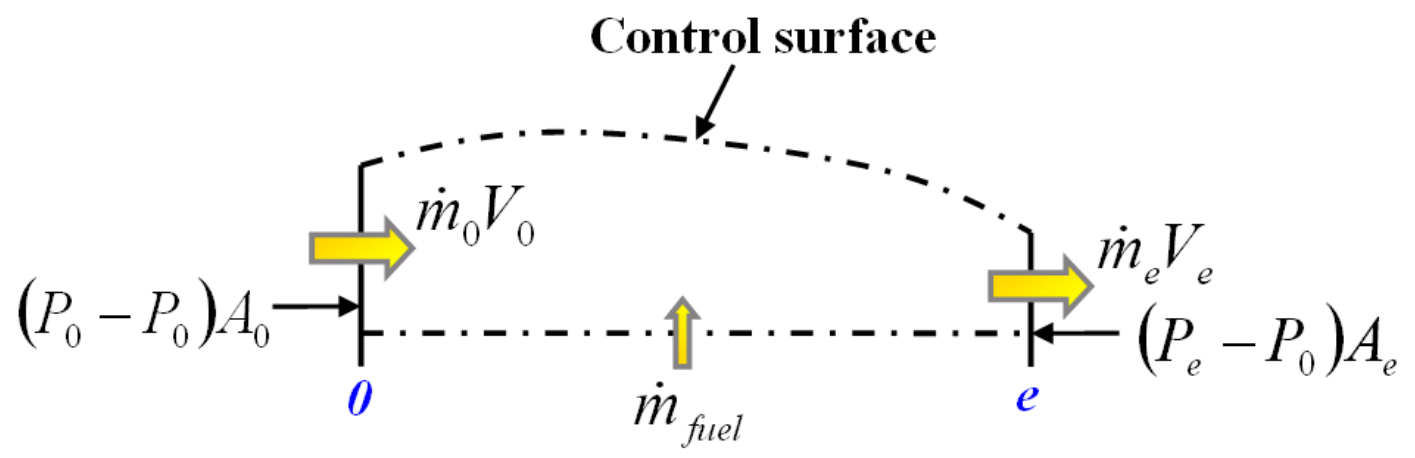

Figure 3.1: Momentum fluxes and pressure forces difference on an aircraft propulsion system

\subsubsection{Thrust Specific Fuel Consumptions}

Other than thrust, we would also like to know how significant the thrust increases compared to the amount of fuel injected. Then, thrust specific fuel 
consumptions is the rate of fuel use by the propulsion system per unit of thrust produced. The uninstalled thrust specific fuel consumption (S) and the installed thrust specific fuel consumption (TSFC) are given by

$$
\begin{gathered}
S=\frac{\dot{m}_{f u e l}}{F} \\
T S F C=\frac{\dot{m}_{f u e l}}{T}
\end{gathered}
$$

\subsubsection{Engine Efficiencies}

The following performance parameters will also be useful, namely, thermal efficiency, propulsive efficiency, and overall efficiency.

\section{Thermal Efficiency $\left(\eta_{t h}\right)$}

The thermal efficiency is defined as the net rate of the kinetic energy gain out of the engine $\dot{W}_{\text {out }}$ divided by the rate of thermal energy available from the fuel $\dot{Q}_{i n}$.

$$
\eta_{t h}=\frac{\dot{W}_{\text {out }}}{\dot{Q}_{\text {in }}}
$$

\section{Propulsive Efficiency $\left(\eta_{p}\right)$}

The propulsive efficiency defines the ratio of the aircraft power (thrust $T \times$ flight velocity $V_{0}$ ) to the power out of the engine $\dot{W}_{\text {out }}$. In other words, it measures how effectively the engine power $\dot{W}_{\text {out }}$ is used to propel the aircraft.

$$
\eta_{p}=\frac{T V_{0}}{\dot{W}_{\text {out }}}
$$

\section{Overall Efficiency $\left(\eta_{o}\right)$}

An overall performance of a propulsion system is given by the product of thermal and propulsive efficiencies. 


$$
\eta_{o}=\eta_{t h} \eta_{p}
$$

\subsection{Engine Cycle and Station Numbering}

The goal of this research is aimed at examining the potential of using an ITB in a turbofan engine at both subsonic and supersonic flight regimes. Currently, a conventional dual-spool, separate-exhaust turbofan engine is chosen as the baseline engine. The complete schematic diagram of this selected engine cycle is shown in Figure 3.2 .

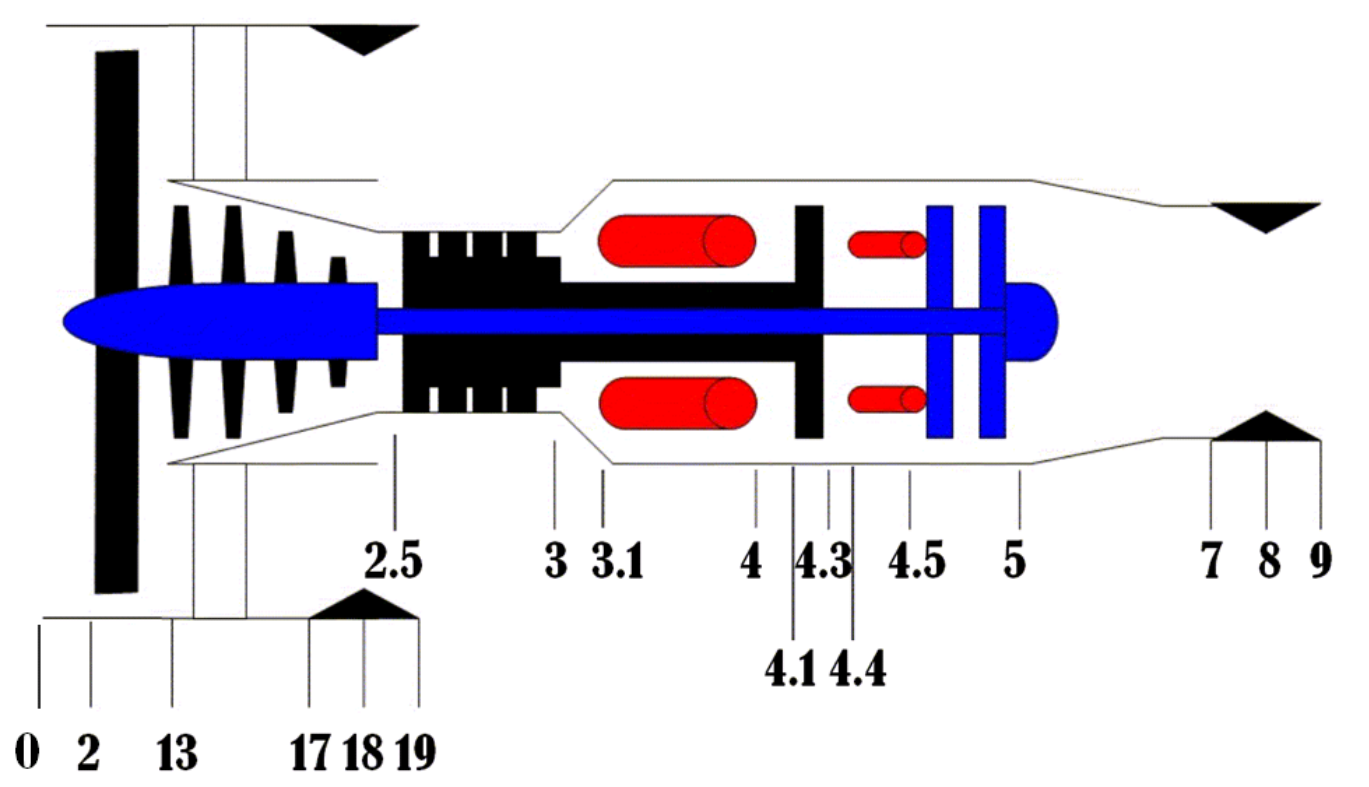

Figure 3.2: Schematic diagram of a turbofan engine with ITB

Figure 3.2 generally illustrate the configuration of a turbofan engine and the station numbering for each component. The station numbers of each component location is in accordance with Aerospace Recommended Practice (APR) 755A [10] and will be used throughout the analysis in this research, including the performance cycle analysis. 
Table 3.1: Definition for each station number

\begin{tabular}{ll}
\hline \hline Station & \multicolumn{1}{c}{ Location } \\
\hline 0 & Far upstream or freestream condition \\
1 & Inlet or diffuser entry \\
2 & Inlet or diffuser exit, fan entry, and low-pressure compressor entry \\
2.5 & Low-pressure compressor exit \\
& High-pressure compressor entry \\
3 & High-pressure compressor exit \\
3.1 & Main burner entry \\
4 & Main burner exit \\
& High-pressure turbine first nozzle guide vane entry \\
4.1 & Coolant mixer entry \\
4.4 & High-pressure turbine first nozzle guide vane exit \\
& ITB entry \\
4.5 & ITB exit \\
5 & Low-pressure turbine entry \\
7 & Low-pressure turbine exit \\
8 & Core exhaust nozzle entry \\
9 & Core exhaust nozzle throat \\
13 & Core exhaust nozzle exit \\
18 & Fan exit \\
\hline \hline
\end{tabular}




\subsection{Gas Model}

In this analysis, it is assumed that the working fluids, i.e., the air and combustion products, are modeled as perfect gases in their thermodynamic equilibrium.

Generally, specific heat at constant pressure $\left(c_{p}\right)$ for air is function of temperature. Also, $c_{p}$ and specific heat ratio $(\gamma)$ for most typical hydrocarbons and air combustion products are functions of temperature and the fuel-air ratios [33]. Therefore, it is necessary to model the variation of $c_{p}$ and $\gamma$ across engine components where the changes are significant, for instance, downstream of burner.

Throughout the analysis, the variation of gas properties with temperature is approximated by assuming constant gas properties, such as $c_{p}, \gamma$, and gas constant $(R)$, at three different sections across the engine core:

- Section 1: components upstream of main burner (i.e., before station 4)

- Section 2: components between station 4 and 4.4

- Section 3: components downstream of ITB (i.e., station 4.5)

The computations of these properties are included into subroutine ENTHALPY. All equations used to calculate these properties are presented in Appendix C.

\subsection{Notations for Compressible Flow}

In the compressible flow system like in the gas turbine engine system, for example, three independent intensive properties are required to fix the thermodynamic state of the gas when it is in motion. At any given point in the compressible flow field, it is achieved by specifying the speed of the gas and 
any two independent properties such as pressure and temperature. However, specifying speed in one-dimensional flow is not always convenient or useful [33].

Consequently, most flow properties used in compressible flow analysis are dependant upon the speed of gas. Any two of these properties, namely, stagnation temperature, stagnation pressure, or Mach number, are adequate to fix a state of a moving gas.

In order to better manipulate the cycle analysis results, it is very important to thoroughly comprehend the physical meanings of some useful quantity notations for compressible flow that are about to be defined below.

First of all, stagnation enthalpy or total enthalpy $\left(h_{t}\right)$ is defined as the enthalpy obtained when a steady flowing fluid is brought to rest in the absence of any heat or work interactions. Applying First Law of Thermodynamics to a steady flowing gas gives total enthalpy:

$$
h_{t}=h+\frac{V^{2}}{2}
$$

where $V$ is the flow velocity.

For calorically perfect gas (i.e., constant $c_{p}$ ), the above equation can be written for stagnation temperature or total temperature $\left(T_{t}\right)$ as

$$
T_{t}=T+\frac{V^{2}}{2 C_{p}}
$$

where $T$ is the static temperature.

Stagnation pressure or total pressure $\left(P_{t}\right)$ is defined as the pressure reached when a steady flowing gas is brought to rest adiabatically and irreversibly. Using the isentropic relation, total pressure is given by 


$$
P_{t}=P\left(\frac{T_{t}}{T}\right)^{\gamma /(\gamma-1)}
$$

Assuming that the gas is a perfect gas, note that the speed of sound $(a)$ is a function of the thermodynamic properties of the gas [3]:

$$
a=\sqrt{\gamma g_{c} R T}
$$

Using this relation for the speed of sound, it yields another important parameter, i.e., Mach number:

$$
M^{2}=\frac{V}{\gamma g_{c} R T}
$$

Note that the Mach number depends on the state of the flowing gas.

With this expression, one can obtain two useful relations that relate total temperature and total pressure to Mach number, such as

$$
\begin{gathered}
P_{t}=P\left(1+\frac{\gamma-1}{2} M^{2}\right)^{\frac{\gamma}{\gamma-1}} \\
T_{t}=T\left(1+\frac{\gamma-1}{2} M^{2}\right)
\end{gathered}
$$

The ratio of total pressures $(\pi)$ and temperatures $(\tau)$ across each component are used extensively in cycle analyses. They are identified by a subscript, as shown in Table 3.2

For instance, compressor total pressure ratio and total temperature ratio are denoted $\pi_{c}$ and $\tau_{c}$, respectively. 
Table 3.2: Subscript definition for total property ratios (referring to station numbers in Figure 3.3)

\begin{tabular}{lll}
\hline \hline Subscript & Component & Station \\
\hline$b$ & Main burner & $3.1 \rightarrow 4$ \\
$c H$ & High-pressure compressor & $2.5 \rightarrow 3$ \\
$c L$ & Low-pressure compressor & $2 \rightarrow 2.5$ \\
$d$ & Inlet or diffuser & $0 \rightarrow 2$ \\
$f$ & Fan & $2 \rightarrow 13$ \\
$i t b$ & ITB & $4.4 \rightarrow 4.5$ \\
$m 1$ & Coolant mixer 1 & $4.1 \rightarrow 4.2$ \\
$m 2$ & Coolant mixer 2 & $4.3 \rightarrow 4.4$ \\
$n$ & Core exhaust nozzle & $7 \rightarrow 9$ \\
$n f$ & Fan exhaust nozzle & $17 \rightarrow 19$ \\
$t H$ & High-pressure turbine & $4 \rightarrow 4.4$ \\
$t L$ & Low-pressure turbine & $4.5 \rightarrow 5$ \\
\hline \hline
\end{tabular}

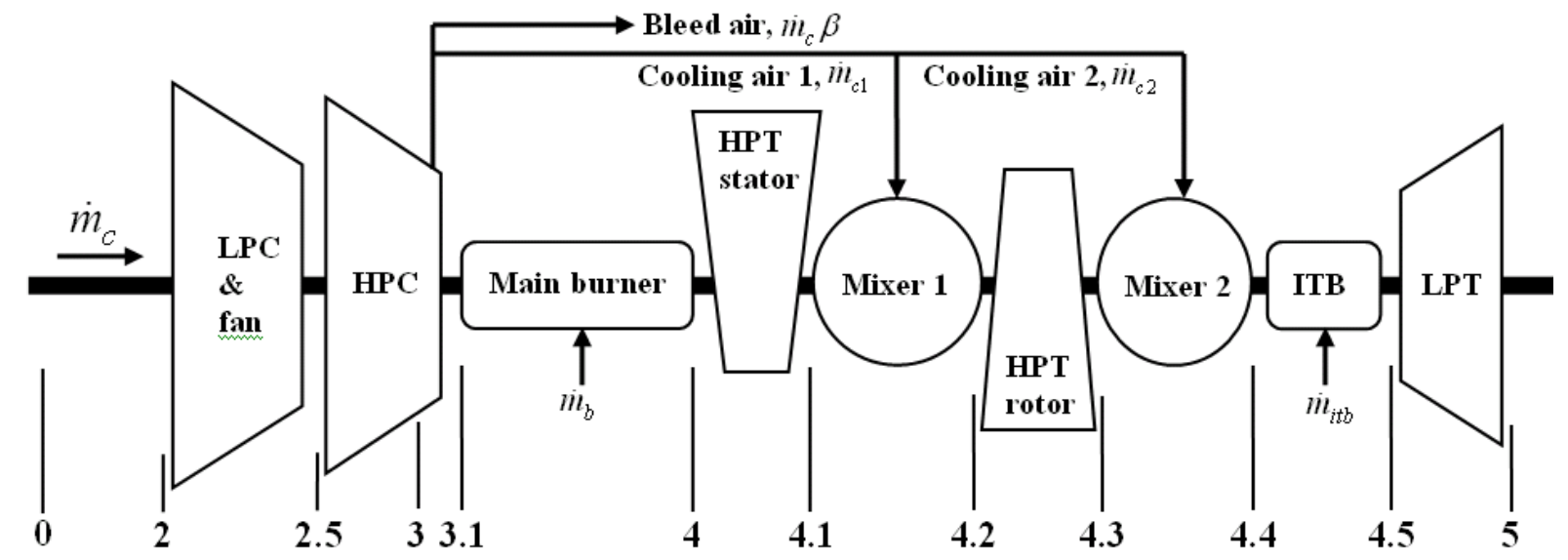

Figure 3.3: Engine layout with cooling air flow 


\subsection{Mass Flow Rates}

Figure 3.3 presents an engine layout with the cooling airflow paths. Readers are able to visualize several air and fuel flow paths within the engine such as (1) the extraction of bleed air and cooling air from the compressor exit, (2) the mixing of cooling air into main stream, and (3) the mixing of injected fuel with air. Since those mass flow rates have major importance in the modeling of engine cycle analysis, it has to be very careful in defining each term. The symbol $\dot{m}$ is used for the mass flow rate with a subscript to denote the type as follows:

\section{Mass ratios:}

Fan bypass ratio:

$$
\alpha=\frac{\dot{m}_{f}}{\dot{m}_{c}}
$$

Bleed air mass fraction:

$$
\beta=\frac{\dot{m}_{b a}}{\dot{m}_{c}}
$$

Cooling air mass fraction:

$$
\begin{aligned}
\epsilon_{1} & =\frac{\dot{m}_{c 1}}{\dot{m}_{c}} \\
\epsilon_{2} & =\frac{\dot{m}_{c 2}}{\dot{m}_{c}}
\end{aligned}
$$

Burner fuel-air ratio:

$$
f_{b}=\frac{\dot{m}_{b}}{\dot{m}_{3.1}}
$$

ITB fuel-air ratio:

$$
f_{i t b}=\frac{\dot{m}_{i t b}}{\dot{m}_{4.4}}
$$


Mass flow rate at each station:

$$
\begin{aligned}
\dot{m}_{0} & =\dot{m}_{c}+\dot{m}_{f} \\
\dot{m}_{3.1} & =\dot{m}_{c}\left(1-\beta-\epsilon_{1}-\epsilon_{2}\right) \\
\dot{m}_{4} & =\dot{m}_{3.1}+\dot{m}_{b} \\
& =\dot{m}_{c}\left(1-\beta-\epsilon_{1}-\epsilon_{2}\right)\left(1+f_{b}\right) \\
\dot{m}_{4.1} & =\dot{m}_{4} \\
\dot{m}_{4.2} & =\dot{m}_{4}+\dot{m}_{c 1} \\
& =\dot{m}_{c}\left[\left(1-\beta-\epsilon_{1}-\epsilon_{2}\right)\left(1+f_{b}\right)+\epsilon_{1}\right] \\
\dot{m}_{4.5} & =\dot{m}_{4.4}+\dot{m}_{i t b} \\
\dot{m}_{4.4} & =\dot{m}_{c .3}\left[\left(1-\beta-\dot{m}_{c 2}\right.\right. \\
& =\dot{m}_{c}\left[\left(1-\beta-\epsilon_{1}-\epsilon_{2}\right)\left(1+f_{b}\right)+\epsilon_{1}+\epsilon_{2}\right] \\
\dot{m}_{4.3} & =\dot{m}_{4.2}
\end{aligned}
$$




\subsection{Component Efficiencies}

\subsubsection{Rotating Machineries}

For rotating machinery components such as compressor and turbine, the losses or real effects are usually denoted by means of efficiencies. The polytropic efficiency $(e)$ is used to relate the stage total pressure ratio $(\pi)$ to total temperature ratio $(\tau)$ because it represents a level of technology rather than the behavior of a given component [34]. For instance, polytropic efficiency for a compressor is defined as [33]:

$$
\eta_{c}=\frac{i d e a l \text { work for compression for a differential pressure change }}{\text { actual work for compression for a dif ferential pressure change }}
$$

With the assumption of constant $e_{c}$, we can obtain the relation between $\tau_{c}$ and $\pi_{c}$ as follow:

$$
\tau_{c}=\pi_{c}^{(\gamma-1) / \gamma e_{c}}
$$

On the other hand, the behavior of a component is represented by isentropic efficiency $(\eta)$, which is used in performance cycle analysis. The compressor isentropic efficiency is defined as follows [33]:

$$
\begin{aligned}
\eta_{c} & =\frac{\text { ideal work of compression for given } \pi_{c}}{\text { actual work of compression for given } \pi_{c}} \\
& =\frac{\pi_{c}^{(\gamma-1) / \gamma}-1}{\pi_{c}^{(\gamma-1) / \gamma e_{c}}-1}
\end{aligned}
$$

Going through similar procedure as the compressor, we obtain turbine isentropic efficiency $\left(\eta_{t}\right)$, turbine polytropic efficiency $\left(e_{t}\right)$, and the relation between $\tau_{t}$ and $\pi_{t}$ as follows: 


$$
\begin{gathered}
\eta_{t}=\frac{\text { actual work of compression for given } \pi_{t}}{\text { ideal work of compression for given } \pi_{t}} \\
\eta_{t}=\frac{1-\pi_{t}^{(\gamma-1) / \gamma e_{t}}}{1-\pi_{t}^{(\gamma-1) / \gamma}} \\
\tau_{t}=\pi_{t}^{(\gamma-1) e_{t} / \gamma}
\end{gathered}
$$

\subsubsection{Combustion components}

Combustion efficiency is defined as the ratio of the actual thermal energy rise to the maximum possible thermal energy rise [34]. In this study, combustion efficiencies for main burner $\left(\eta_{b}\right)$ and ITB $\left(\eta_{i t b}\right)$ are set as input parameters.

\subsubsection{Power transmission components}

Mechanical efficiency is defined as the ratio of mechanical energy output to the mechanical energy input. It is usually used to account for any losses due to bearing friction, seal drag, windage etc while transmitting mechanical power by means of shafts. These terms, i.e., $\eta_{m L}$ and $\eta_{m H}$, represent the mechanical efficiencies for low-pressure and high-pressure spool, respectively. 


\section{Chapter 4}

\section{Parametric Cycle Analysis of a Turbofan Engine with ITB}

\subsection{Introduction}

In the previous chapter, the need for an analytical tool to accurately estimate the engine performance of a dual-spool turbofan with ITB has been identified. In this chapter, the design parameters, assumptions, and modeling approaches incorporated in the parametric cycle analysis program are presented and discussed.

As clearly shown in Figure 1.7, engine design starts with the parametric cycle analysis, followed by the performance cycle analysis, which is presented in the Chapter 5. These two analyses are done by treating each stream entering engine as the one-dimensional flow of a perfect gas. As the working fluids (such as air and products of combustion) flow across the engine, their thermodynamic properties change, which also characterize the behaviors of each engine component.

The objective of parametric cycle analysis is to estimate the performance parameters (primarily specific thrust and thrust specific fuel consumption) 
in terms of design limitation (maximum turbine inlet temperature), flight conditions (such as flight Mach condition, ambient temperature and pressure), and design choices [such as compressor pressure ratio (CPR), fan pressure ratio (FPR), fan bypass ratio (FBR) etc.] [34]. In other words, it determines how the engine performance varies with changes in design limits, flight conditions, and design choices.

Since geometry is not included in the parametric study, one can easily specify engine component characteristics over a desired operating range for a particular application. Performance results are then used to search for possible operating range for each engine component for an optimum design choice. By doing so, however, the resulting plots of specific thrust (one of primary performance parameters) versus, say, fan bypass ratio are not representing the behavior of a specific engine. Each point on such plots denotes a different engine configuration or a so-called design-point engine. Therefore, parametric analysis is sometimes called design-point analysis, or on-design analysis.

At the end of the parametric analysis, one should be able to identify the possible range and the combinations of each design choice that promise to give the best performance at each flight condition.

\subsection{Assumptions}

The following assumptions are summarized:

1. The working fluid is air and products of combustion, which behaves as perfect gases.

2. Perfect gas upstream of main burner (station 4) with constant properties $\gamma_{c}, R_{c}, c_{p c}$

3. Perfect gas between station 4 and 4.5 with constant properties $\gamma_{t}, R_{t}, c_{p t}$. 
4. Perfect gas downstream of inter-stage burner (station 4.5) with constant properties $\gamma_{i t b}, R_{i t b}, c_{p i t b}$.

5. All components are adiabatic.

6. The efficiencies of the high-pressure compressor (HPC), low-pressure compressor (LPC), fan, HPT, and LPT are described through the use of polytropic efficiencies, i.e., $e_{c H}, e_{c L}, e_{f}, e_{t H}$, and $e_{t L}$, respectively.

\subsubsection{Turbine Cooling}

For better performance prediction, a turbine cooling model is incorporated into the engine analysis. The approximate turbine cooling model was first presented in Oates [39]. A portion of the cooling air drawn off at the HPC exit (station 3) is used to cool the HPT stator $\left(\epsilon_{1}\right)$ and the remainder $\left(\epsilon_{2}\right)$ to cool HPT rotor. It is assumed that the mixing of two cooling air with the mainstream occurs after the first stator and the last rotor. The cooling airflows are modeled as being fully mixed in coolant mixer 1 and coolant mixer 2, respectively, and there is no total pressure losses. In order to reduce the further performance loss due to cooling air, it is assumed that there is no cooling air required for LPT.

Figure 4.1 shows the correlation curves for the amount of cooling air required for each stator and rotor, as given in Walsh and Fletcher [54]. All curves are based upon the HPT stator outlet temperature $\left(T_{t 4.1}\right)$. For an aircraft engine, the stateof-the-art maximum allowable turbine blade temperature (by year 2000) is 1370K [55]. Therefore, it is reasonable to use the high-tech cooling curve and to assume that the cooling is only required when the turbine inlet temperature exceeds 1300K. Accordingly, the equations for the high-tech cooling curves of the stator and rotor, respectively, are shown below:

$$
\epsilon_{1}=0.0167 T_{t 4.1}-19.97(\%)
$$




$$
\epsilon_{2}=0.0343 T_{t 4.1}-46.362(\%)
$$

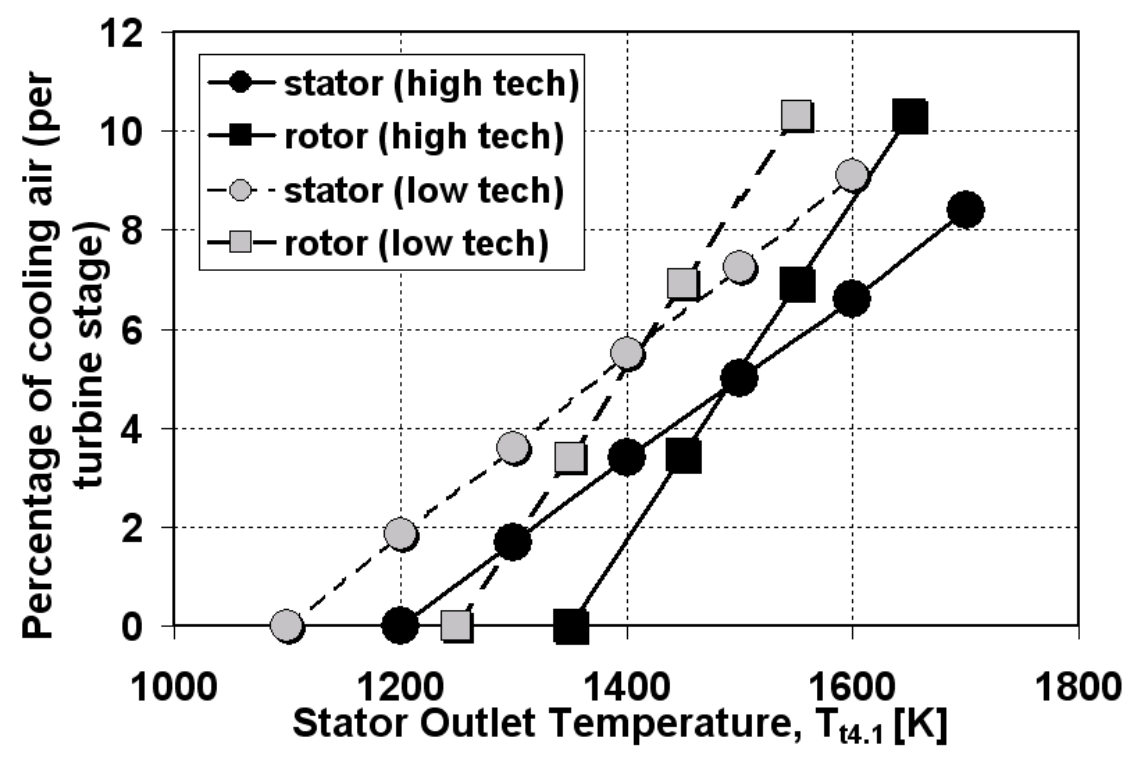

Figure 4.1: Correlation curves of cooling air percentage (stator and rotor) vs. stator outlet temperature $\left(T_{t 4.1}\right)$ [54]

\subsection{Engine Component Performance}

\subsubsection{Free Stream}

In order to employ total (or stagnation) property ratios, it is necessary to define the total/static temperature $\left(\tau_{r}\right)$ and pressure ratios $\left(\pi_{r}\right)$ of the free stream according to section 3.4 as follows:

$$
\begin{gathered}
\tau_{r}=\frac{T_{t 0}}{T_{0}}=1+\frac{\gamma_{c}-1}{2} M_{0}^{2} \\
\pi_{r}=\frac{P_{t 0}}{P_{0}}=\left(1+\frac{\gamma_{c}-1}{2} M_{0}^{2}\right)^{\gamma_{c} /\left(\gamma_{c}-1\right)}
\end{gathered}
$$

The ambient condition is first specified by users in term of altitude $(h)$. 
Then, the ambient temperature $T_{0}$ and pressure $P_{0}$ are determined from the US Standard Atmosphere 1976 data [34] according to the calculation procedures as shown in Appendix B.

\subsubsection{Inlet and Diffuser}

The primary function of the inlet or diffuser is to bring the incoming air required by the engine from freestream conditions to the conditions required at the entrance of fan and compressor with minimum total losses. The high-speed (subsonic or supersonic) incoming flow needs to undergo diffusion process in a divergent duct, leading to a reduction in flow velocity and a rise in static pressure. Typically, uniform flow of air at Mach number of 0.5 is the best possible entry condition for fan and compressor [33].

The inlet total pressure is defined as the product of the ram pressure ratio $\left(\pi_{r}\right)$ and the diffuser pressure ratio $\left(\pi_{d}\right)$. Pressure losses occur due to the friction within the inlet wall. Consequently, $\pi_{d}$ is always less than one. In the cycle analysis, $\pi_{d}$ is normally assumed to be constant in subsonic inlets, which is equal to $\pi_{d \max }$ (i.e., the total pressure ratio due to friction). In supersonic flight, the pressure losses cause shock waves which produce greater pressure losses. Therefore, the following relationship is employed to account for the variation of $\pi_{d}$ with flight Mach number:

$$
\pi_{d}=\pi_{d \max } \eta_{R s p e c}
$$

According to the Military Specification 5008B [36], the following relations is obtained for $\eta_{\text {Rspec }}$ : 


$$
\eta_{\text {Rspec }}=\left\{\begin{array}{lll}
1 & \text { for } & M_{0} \leq 1 \\
1-0.075\left(M_{0}-1\right)^{1.35} & \text { for } & 1<M_{0}<5 \\
\frac{800}{M_{0}^{4}+935} & \text { for } & M_{0}>5
\end{array}\right.
$$

After passing through the inlet and diffuser, the air stream entering the turbofan engine will flow through the engine core and the fan separately. The engine core components in sequence are LPC, HPC, main burner, HPT, ITB, LPT, and core exhaust nozzle. Meanwhile, surrounding the core engine is the fan assembly, which include fan, fan duct and fan exhaust nozzle.

\subsubsection{Low-pressure and High-pressure Compressors}

Commonly, axial-flow compressor is the type of compressor used in today's turbofan engine. It consists of a series of stages, each stage comprising a row of rotor blades followed by a row of stator blades. The working fluid is initially accelerated by the rotor blades, and then decelerated in the stator blade passages. Consequently, the primary function of this arrangement is to convert the kinetic energy transferred from the rotor to an increase in pressure of the gas stream while absorbing minimum shaft power possible.

In parametric cycle analysis, the terms $\pi_{c L}$ and $\pi_{c H}$ denote the total pressure ratios across the LPC and HPC, respectively. In fact, they are the design parameters and their values are specified by users. However, the value of LPC pressure ratio is not always equal to the user-defined value $\pi_{c L}$. In a turbofan engine, LPC is located immediately downstream of the fan. Therefore, whenever the specified $\pi_{c L}$ is smaller than $\pi_{f}$, the following condition is applied instead: $\pi_{c L}=\pi_{f}$.

The component efficiencies for these compressors have been discussed and presented in Section 3.6. The total temperature ratios across LPC and HPC are 
related to their total pressure ratios through constant polytropic efficiencies $\left(e_{c H}\right.$ and $e_{c L}$ ), as given by

$$
\begin{gathered}
\tau_{c H}=\pi_{c H}^{\left(\gamma_{c}-1\right) /\left(\gamma_{c} e_{c H}\right)} \\
\tau_{c L}=\pi_{c L}^{\left(\gamma_{c}-1\right) /\left(\gamma_{c} e_{c L}\right)}
\end{gathered}
$$

\subsubsection{Main burner and ITB}

Ideally, both main burner and ITB go through an isobaric (constant pressure) combustion process. Consequently, both components are described by a similar set of equations in the program.

The term $\tau_{\lambda}$ is defined and used in terms of design limitations such as maximum allowable turbine inlet total temperature. It denotes a ratio of total enthalpy of the burner exit to the ambient enthalpy:

Main burner

$$
\tau_{\lambda-b}=\frac{\left(c_{p} T_{t}\right)_{\text {burner exit }}}{\left(c_{p} T\right)_{0, \text { ambient }}}
$$

ITB

$$
\tau_{\lambda-i t b}=\frac{\left(c_{p} T_{t}\right)_{\text {ITB exit }}}{\left(c_{p} T\right)_{0, \text { ambient }}}
$$

The combustion efficiencies for main burner $\left(\eta_{b}\right)$ and ITB are introduced early in Section 3.6. As far as the chemistry model is concerned, all combustions are assumed to be almost complete. And fortunately, most current burners possess combustion efficiency in excess of $99 \%$. Even the new-concept UltraCompact Combustor (UCC) at AFRL operates at 95-99\% combustion efficiency over a wide range of operating conditions [56]. So, this approximation should 
not affect the accuracy of results too much.

The following procedures are shown to determine the fuel-air ratio for main burner $\left(f_{b}\right)$ and $\operatorname{ITB}\left(f_{i t b}\right)$ :

\section{Main burner:}

Apply First Law of Thermodynamics and ideal gas relation to the main burner:

$$
\dot{m}_{3.1} c_{p c} T_{t 3.1}+\dot{m}_{b} \eta_{b} h_{P R-b}=\dot{m}_{4} c_{p t} T_{t 4}
$$

Rearranging gives:

$$
c_{p c} T_{t 3.1}+f_{b} \eta_{b} h_{P R-b}=\left(1+f_{b}\right) c_{p t} T_{t 4}
$$

Multiplying the above equation with $\frac{1}{C_{p c} T_{0}}$, and solving for $f_{b}$ :

$$
f_{b}=\frac{\tau_{\lambda-b}-\tau_{r} \tau_{d} \tau_{c L} \tau_{c H}}{\eta_{b} h_{P R} /\left(c_{p c} T_{0}\right)-\tau_{\lambda-b}}
$$

\section{ITB:}

Similarly, apply First Law of Thermodynamics and ideal gas relation to the ITB:

$$
\dot{m}_{4.4} c_{p t} T_{t 4.4}+\dot{m}_{i t b} \eta_{i t b} h_{P R-i t b}=\dot{m}_{4.5} c_{p i t b} T_{t 4.5}
$$

Rearranging the above equation gives:

$$
c_{p t} T_{t 4.4}+f_{i t b} \eta_{i t b} h_{P R-i t b}=\left(1+f_{i t b}\right) c_{p i t b} T_{t 4.5}
$$


Multiplying the above equation with $\frac{1}{c_{p c} T_{0}}$, and solving for $f_{i t b}$ gives:

$$
f_{i t b}=\frac{\tau_{\lambda-i t b}-\tau_{\lambda-b} \tau_{m 1} \tau_{m 1} \tau_{t H}}{\eta_{i t b} h_{P R} /\left(c_{p c} T_{0}\right)-\tau_{\lambda-i t b}}
$$

For details on how these two expressions for fuel-air ratio are integrated into the program, please refer to Appendix C.

\subsubsection{Turbine Coolant Mixer}

It is assumed that the total pressure loss due to the mixing process in the first coolant mixer (station 4.1 to 4.2 ) is negligible. Then, applying first law energy balance to first coolant mixer yields:

$$
\dot{m}_{4} c_{p t} T_{t 4}+\dot{m}_{c 1} c_{p c} T_{t 3}=\dot{m}_{4.1} c_{p t} T_{t 4.1}
$$

Rearranging above equation gives

$$
\tau_{m 1}=\frac{\left(1-\beta-\epsilon_{1}-\epsilon_{2}\right)\left(1+f_{b}\right)+\epsilon_{1} \tau_{r} \tau_{d} \tau_{c} / \tau_{\lambda-b}}{\left(1-\beta-\epsilon_{1}-\epsilon_{2}\right)\left(1+f_{b}\right)+\epsilon_{1}}
$$

Likewise, applying first law energy balance to second coolant mixer (station 4.3 to 4.4 ) yields:

$$
\dot{m}_{4.3} c_{p t} T_{t 4.3}+\dot{m}_{c 2} c_{p c} T_{t 3}=\dot{m}_{4.4} c_{p t} T_{t 4.4}
$$

Rearranging above equation gives

$$
\tau_{m 2}=\frac{\left(1-\beta-\epsilon_{1}-\epsilon_{2}\right)\left(1+f_{b}\right)+\epsilon_{1}+\epsilon_{2} \tau_{r} \tau_{d} \tau_{c} /\left(\tau_{\lambda-b} \tau_{m 1} \tau_{t H}\right)}{\left(1-\beta-\epsilon_{1}-\epsilon_{2}\right)\left(1+f_{b}\right)+\epsilon_{1}+\epsilon_{2}}
$$




\subsubsection{High-pressure Turbine}

A turbine comprises a row of nozzle guide vanes (or stators) followed by a row of rotor blades mounted on rotating discs. Turbine has most likely fewer stages compared to the compressor. In contrast to compressor, turbine extracts power from the high pressure and high temperature gas stream. The extracted power is used to drive compressors and fan in this dual-spool turbofan engine through two shafts.

In high-pressure spool, HPT and HPC are connected by a single shaft and the power extracted by the HPT will be completely consumed by the HPC:

$$
\dot{W}_{c H}=\dot{W}_{t H}
$$

Applying First Law of Thermodynamics and ideal gas relation to the above relation yields:

$$
\dot{m}_{c} c_{p t}\left(T_{t 3}-T_{t 2.5}\right)=\dot{m}_{4.1} c_{p t} \eta_{m H}\left(T_{t 4.1}-T_{t 4.3}\right)
$$

Multiplying the above equation with $\frac{1}{c_{p c} T_{0}}$ gives

$$
\left(\tau_{c H}-1\right)=\left[\left(1-\beta-\epsilon_{1}-\epsilon_{2}\right)\left(1+f_{b}\right)+\epsilon_{1}\right] \frac{c_{p t} T_{t 4}}{c_{p c} T_{t 3.1}} \tau_{m 1} \tau_{c H} \eta_{m H}\left(1-\tau_{t H}\right)
$$

Solving for $\tau_{t H}$ gives

$$
\tau_{t H}=1-\frac{\left(\tau_{c H}-1\right) \tau_{c L} \tau_{r}}{\left[\left(1-\beta-\epsilon_{1}-\epsilon_{2}\right)\left(1+f_{b}\right)+\epsilon_{1}\right] \tau_{\lambda-b} \tau_{m 1} \eta_{m H}}
$$


If there is no bleed air and no turbine cooling, the above equation becomes:

$$
\tau_{t H}=1-\frac{\left(\tau_{c H}-1\right) \tau_{c L} \tau_{r}}{\left(1+f_{b}\right) \tau_{\lambda-b} \eta_{m H}}
$$

The component efficiency for a turbine has been discussed and presented in Section 3.6. The total pressure ratio across the HPT $\left(\pi_{t H}\right)$ is related to its total temperature ratio $\left(\tau_{t H}\right)$ through the constant polytropic efficiency $\left(e_{t H}\right)$, as given by

$$
\pi_{t H}=\tau_{t H}^{\gamma_{t} /\left[\left(\gamma_{t}-1\right) e_{t H}\right]}
$$

According to the relationships derived in Section 3.6, the high-pressure turbine isentropic efficiency can be written as

$$
\eta_{t H}=\frac{1-\tau_{t H}}{1-\tau_{t H}^{1 / e_{t H}}}
$$

\subsubsection{Low-pressure Turbine}

Similarly, LPC, fan and LPT are connected through another shaft in a lowpressure spool. It is assumed that all power extracted by the LPT will be used to drive both LPC and fan.

$$
\dot{W}_{c L}+\dot{W}_{f}=\dot{W}_{t L}
$$

Again, applying First Law of Thermodynamics and ideal gas relation to the above relation yields

$$
\dot{m}_{c} c_{p c}\left(T_{t 2.5}-T_{t 2}\right)+\dot{m}_{f} c_{p c}\left(T_{t 13}-T_{t 2}\right)=\dot{m}_{4.5} c_{p i t b} \eta_{m L}\left(T_{t 4.5}-T_{t 5}\right)
$$


Multiplying the above equation with $\frac{1}{c_{p c} T_{t 2}}$ gives

$$
\begin{aligned}
\left(\tau_{c L}-1\right)+\alpha\left(\tau_{f}-1\right)= & {\left[\left(1-\beta-\epsilon_{1}-\epsilon_{2}\right)\left(1+f_{b}\right)+\epsilon_{1}+\epsilon_{2}\right] \frac{c_{p t}}{c_{p c}} \frac{T_{t 4.5}}{T_{t 4.4}} } \\
& \tau_{m 1} \tau_{t H} \tau_{m 2} \frac{T_{t 4}}{T_{t 3.1}} \tau_{c H} \tau_{c L}\left(1-\tau_{t L}\right) \eta_{m L}
\end{aligned}
$$

Solving for $\tau_{t L}$ gives

$$
\tau_{t L}=1-\frac{\left[\left(\tau_{c L}-1\right)+\alpha\left(\tau_{f}-1\right)\right] \tau_{r}}{\left[\left(1-\beta-\epsilon_{1}-\epsilon_{2}\right)\left(1+f_{b}\right)+\epsilon_{1}+\epsilon_{2}\right]\left(1+f_{i t b}\right) \tau_{\lambda-i t b} \eta_{m L}}
$$

If there is no bleed air and no turbine cooling, the above equation becomes:

$$
\tau_{t L}=1-\frac{\left[\left(\tau_{c L}-1\right)+\alpha\left(\tau_{f}-1\right)\right] \tau_{r}}{\left(1+f_{b}\right)\left(1+f_{i t b}\right) \tau_{\lambda-i t b} \eta_{m L}}
$$

The total pressure ratio across the LPT $\left(\pi_{t L}\right)$ is related to its total temperature ratio $\left(\tau_{t L}\right)$ through the constant polytropic efficiency $\left(e_{t L}\right)$, as given by

$$
\pi_{t L}=\tau_{t L}^{\gamma_{i t b} /\left[\left(\gamma_{i t b}-1\right) e_{t L}\right]}
$$

The LPT isentropic efficiency is written as

$$
\eta_{t L}=\frac{1-\tau_{t L}}{1-\tau_{t L}^{1 / e_{t L}}}
$$




\subsubsection{Exhaust Nozzles}

Exhaust nozzle is used to increase the velocity of the exhaust gas stream through an expansion process before discharging air to the ambient. The most important factor that affects the expansion process is the pressure ratio across the nozzle. When the exit pressure $\left(P_{e}\right)$ is equal to the ambient pressure $\left(P_{0}\right)$, a complete expansion occurs, and thus maximum thrust is obtained. There are two types of nozzles in this analysis, i.e., convergent-divergent nozzle and convergent only nozzle.

For the convergent-divergent nozzle, the flow is always assumed to be choked at the throat, where the cross-sectional area of the nozzle duct is at minimum.

\section{Convergent-divergent nozzle}

\section{Core Exhaust Nozzle}

The exit Mach number $M_{9}$ is the most important parameter in describing the core exhaust nozzle's behavior. One can determine $M_{9}$ from the total pressure and Mach number relation as shown earlier in section 3.4 ;

$$
P_{t 9}=P_{9}\left(1+\frac{\gamma_{i t b}-1}{2} M_{9}^{2}\right)^{\frac{\gamma_{i t b}}{\gamma_{i t b}-1}}
$$

Rearranging the above equation gives an expression for $M_{9}$ :

$$
M_{9}=\sqrt{\frac{2}{\gamma_{i t b}-1}\left[\left(\frac{P_{t 9}}{P_{9}}\right)^{\left(\gamma_{i t b}-1\right) / \gamma_{i t b}}-1\right]}
$$

where

$$
\begin{gathered}
\frac{P_{t 9}}{P_{9}}=\frac{P_{0}}{P_{9}} \pi_{r} \pi_{d} \pi_{c L} \pi_{c H} \pi_{b} \pi_{t H} \pi_{i t b} \pi_{t L} \pi_{n} \\
\text { if } M_{9}>1, \text { then } M_{8}=1 \text {, else } M_{8}=M_{9} .
\end{gathered}
$$


The loss due to the expansion process is reflected in the value of total pressure ratio across core nozzle $\pi_{n}$. Assuming the core nozzle is adiabatic, the total temperature ratio $\tau_{n}$ is therefore equal to unity.

\section{Fan Exhaust Nozzle}

Similar to core exhaust nozzle, the exit Mach number $M_{19}$ is the most important parameter of the fan exhaust nozzle. Again, one can determine $M_{19}$ from the total pressure and Mach number relation:

$$
P_{t 19}=P_{19}\left(1+\frac{\gamma_{c}-1}{2} M_{19}^{2}\right)^{\frac{\gamma_{c}}{\gamma_{c}-1}}
$$

Rearranging the above equation gives an expression for $M_{19}$ :

$$
M_{19}=\sqrt{\frac{2}{\gamma_{c}-1}\left[\left(\frac{P_{t 19}}{P_{19}}\right)^{\left(\gamma_{c}-1\right) / \gamma_{c}}-1\right]}
$$

where

$$
\begin{gathered}
\frac{P_{t 19}}{P_{19}}=\frac{P_{0}}{P_{19}} \pi_{r} \pi_{d} \pi_{f} \pi_{n f} \\
\text { if } M_{19}>1 \text {, then } M_{18}=1 \text {, else } M_{18}=M_{19} .
\end{gathered}
$$

The total pressure ratio across fan exhaust nozzle $\pi_{n f}$ is used to account for any loss due to the expansion process through the nozzle. Assuming the fan nozzle is adiabatic, the total temperature ratio $\tau_{n f}$ is always equal to unity.

\section{Convergent nozzle}

As for convergent only nozzle, it has a fixed throat area. The flow across the throat area will choke when the exhaust total pressure/ambient 
static pressure ratio $\left(P_{t e} / P_{0}\right)$ is equal to or larger than $\left(\frac{\gamma+1}{2}\right)^{\gamma /(\gamma-1)}$. Then, the exit Mach number is equal to one (i.e., the sonic speed). On the other hand, when the flow is unchoked, i.e., $\frac{P_{t e}}{P_{0}}<\left(\frac{\gamma+1}{2}\right)^{\gamma /(\gamma-1)}$, the exit pressure is equal to the ambient pressure $\left(P_{e}=P_{0}\right)$, and the exit Mach number is subsonic.

\subsection{Overall Performance}

Cycle analysis is applied to both the engine core stream and fan bypass stream separately as listed below.

\subsubsection{Fan Bypass Stream}

Fan is usually attached to the first stage of low-pressure compressor in a turbofan engine. It has a lower pressure ratio compared to core compressors. At the downstream of the fan, the flow is immediately split into the cold (or bypass), and the hot (or core) stream.

As the name cold implies, there is no combustion taking place in the fan duct. Therefore, the fan bypass stream has the same gas properties $\left(c_{p c}, \gamma_{c}, R_{c}\right)$ as the engine core stream up to the main burner.

Uninstalled thrust of fan stream, $F_{f}$, is given by

$$
F_{f}=\frac{\dot{m}_{f}}{g_{c}}\left(V_{19}-V_{0}\right)-\left(P_{19}-P_{0}\right) A_{19}
$$

Expanding and rearranging the above equation gives

$$
\frac{F_{f}}{\dot{m}_{f}}=\frac{a_{0}}{g_{c}}\left[\frac{V_{19}}{a_{0}}-M_{0}+\frac{T_{19} / T_{0}}{V_{19} / a_{0}} \frac{1}{\gamma_{c}}\left(1-\frac{P_{0}}{P_{19}}\right)\right]
$$


where

$$
\begin{gathered}
\left(\frac{V_{19}}{a_{0}}\right)^{2}=\frac{a_{19}^{2} M_{19}^{2}}{a_{0}^{2}}=\frac{T_{19}}{T_{0}} M_{19}^{2} \\
\frac{T_{19}}{T_{0}}=\frac{T_{t 19} / T_{0}}{T_{t 19} / T_{19}}=\frac{T_{t 19} / T_{0}}{\left(P_{t 19} / P_{19}\right)^{\left(\gamma_{c}-1\right) / \gamma_{c}}} \\
\frac{T_{t 19}}{T_{0}}=\tau_{r} \tau_{f} \tau_{n f}
\end{gathered}
$$

\subsubsection{Engine Core Stream}

The uninstalled thrust of core stream, $F_{c}$, is given by

$$
F_{c}=\frac{1}{g_{c}}\left(\dot{m}_{9} V_{9}-\dot{m}_{0} V_{0}\right)+\left(P_{9}-P_{0}\right) A_{9}
$$

Expanding and rearranging the above equation gives:

$$
\begin{aligned}
\frac{F_{c}}{\dot{m}_{c}}= & \frac{a_{0}}{g_{c}}\left\{\left[\left(1-\beta-\epsilon_{1}-\epsilon_{2}\right)\left(1+f_{b}\right)+\epsilon_{1}+\epsilon_{2}\right]\left(1+f_{i t b}\right) \frac{V_{9}}{a_{0}}-M_{0}\right. \\
& \left.+\left[\left(1-\beta-\epsilon_{1}-\epsilon_{2}\right)\left(1+f_{b}\right)+\epsilon_{1}+\epsilon_{2}\right]\left(1+f_{i t b}\right) \frac{R_{i t b}}{R_{c}} \frac{T_{9} / T_{0}}{V_{9} / a_{0}} \frac{1}{\gamma_{c}}\left(1-\frac{P_{0}}{P_{9}}\right)\right\}
\end{aligned}
$$

where

$$
\begin{gathered}
\left(\frac{V_{9}}{a_{0}}\right)^{2}=M_{9}^{2} \frac{\gamma_{i t b}}{\gamma_{c}} \frac{R_{i t b}}{R_{c}} \frac{T_{9}}{T_{0}} \\
\frac{T_{9}}{T_{0}}=\frac{T_{t 9} / T_{0}}{T_{t 9} / T_{9}}=\frac{T_{t 9} / T_{0}}{\left(P_{t 9} / P_{9}\right)^{\left(\gamma_{i t b}-1\right) / \gamma_{i t b}}} \\
\frac{T_{t 9}}{T_{0}}=\frac{c_{p c}}{c_{p i t b}} \tau_{\lambda-b} \tau_{t L} \tau_{n}
\end{gathered}
$$


If there is no bleed air and no turbine cooling, equation 4.49 then becomes

$$
\frac{F_{c}}{\dot{m}_{c}}=\frac{a_{0}}{g_{c}}\left\{\left[\left(1+f_{b}\right)\left(1+f_{i t b}\right) \frac{V_{9}}{a_{0}}-M_{0}\right]+\left(1+f_{b}\right)\left(1+f_{i t b}\right) \frac{R_{t}}{R_{c}} \frac{T_{9} / T_{0}}{V_{9} / a_{0}} \frac{1}{\gamma_{c}}\left(1-\frac{P_{0}}{P_{9}}\right)\right\}
$$

\section{Aircraft Performance Parameters}

The specific thrust $(S T)$ or total uninstalled thrust per unit mass flow rate intake is given by:

$$
\frac{F}{\dot{m}_{0}}=\frac{F_{c}+F_{f}}{\dot{m}_{c}+\dot{m}_{f}}=\frac{\frac{F_{c}}{\dot{m}_{c}}+\alpha \frac{F_{f}}{\dot{m}_{f}}}{1+\alpha}
$$

The thrust specific fuel consumption $(S)$ is given by

$$
S=\frac{\dot{m}_{b}+\dot{m}_{i t b}}{F}
$$

Expanding equation 4.55 yields

$$
S=\frac{\left(1-\beta-\epsilon_{1}-\epsilon_{2}\right) f_{b}+\left[\left(1-\beta-\epsilon_{1}-\epsilon_{2}\right)\left(1+f_{b}\right)+\epsilon_{1}+\epsilon_{2}\right] f_{i t b}}{\frac{F_{c}}{\dot{m}_{c}}+\alpha \frac{F_{f}}{\dot{m}_{f}}}
$$

The power out of a turbofan engine with an ITB is given by

$$
\dot{W}_{\text {out }}=\frac{1}{2 g_{c}}\left[\left(\dot{m}_{9} V_{9}^{2}-\dot{m}_{c} V_{0}^{2}\right)+\dot{m}_{f}\left(V_{19}^{2}-V_{0}^{2}\right)\right]
$$

Rearranging the above equation gives:

$$
\frac{\dot{W}_{\text {out }}}{\dot{m}_{c}}=\frac{1}{2 g_{c}}\left\{\left\{\left[\left(1-\beta-\epsilon_{1}-\epsilon_{2}\right)\left(1+f_{b}\right)+\epsilon_{1}+\epsilon_{2}\right] V_{9}^{2}-V_{0}^{2}\right\}+\alpha\left(V_{19}^{2}-V_{0}^{2}\right)\right\}
$$

The rate of thermal energy released from the main burner and ITB can be 
written as

$$
\dot{Q}_{i n}=\dot{m}_{b} h_{P R-b} \eta_{b}+\dot{m}_{i t b} h_{P R-i t b} \eta_{i t b}
$$

Rearranging equation 4.59 gives:

$\frac{\dot{Q}_{i n}}{\dot{m}_{c}}=\left(1-\beta-\epsilon_{1}-\epsilon_{2}\right) f_{b} h_{P R-b} \eta_{b}+\left[\left(1-\beta-\epsilon_{1}-\epsilon_{2}\right)\left(1+f_{b}\right)+\epsilon_{1}+\epsilon_{2}\right] f_{i t b} h_{P R-i t b} \eta_{i t b}$

The thermal efficiency can be written as:

$$
\eta_{t h}=\frac{\frac{\dot{W}_{\text {out }}}{\dot{m}_{c}}}{\frac{\dot{Q}_{\text {in }}}{\dot{m}_{c}}}
$$

Meanwhile, propulsive efficiency can be written as

$$
\eta_{p}=\frac{\left(\frac{F_{c}}{\dot{m}_{c}}+\alpha \frac{F_{f}}{\dot{m}_{f}}\right) V_{0}}{\frac{\dot{W}_{\text {out }}}{\dot{m}_{c}}}
$$

Unlike the ground-based gas turbine, the aircraft gas turbine is mounted on a moving vehicle. Therefore, the overall efficiency is a product of the thermal and propulsive efficiencies.

$$
\eta_{O}=\eta_{t h} \times \eta_{p}
$$

\subsection{Computer Code}

The Visual Basic Application program, Excel macrocode, and Excel neuron cells are used to facilitate a widely-used Excel software to plot the engine performance versus several engine design parameters and limitation. This on-design cycle analysis program computes and plots the data sequentially without forcing

users to open other types of plotting programs. An user's manual on how to use the program and its troubleshooting are included in the Appendix G. 


\subsubsection{Code Validation}

This on-design cycle analysis code is developed for evaluating the effect of adding an ITB as a secondary combustor on a conventional turbofan engine. The development of this kind of new engine is still in its preliminary design

phase. Consequently, the experimental data is not available for the purpose of code validation.

Despite the lack of real field data, the predicted result of a conventional turbofan engine using this Excel code was compared to the ONX program (i.e., a parametric cycle analysis program), which is an integrated part of the AEDsys suite co-developed by Mattingly, Heiser and Pratt [34]. The AEDsys software has been developed and refined over a period of more than 20 years, and has become a formidable capability.

The performance of a conventional two-spool, separate-exhaust turbofan engine versus flight Mach number $\left(M_{0}\right)$ is computed using both ONX program and Excel code. As clearly shown in Figures 4.2 through 4.2 , the comparison of the engine performance was found to be consistent.

AEDsys suite requires user to input constant values for $c_{p}$ while the Excel code calculates $c_{p}$ as a function of temperature at each particular section. It is believed that this difference causes a slightly downward shift on the performance curves as shown in Figures 4.2 . 

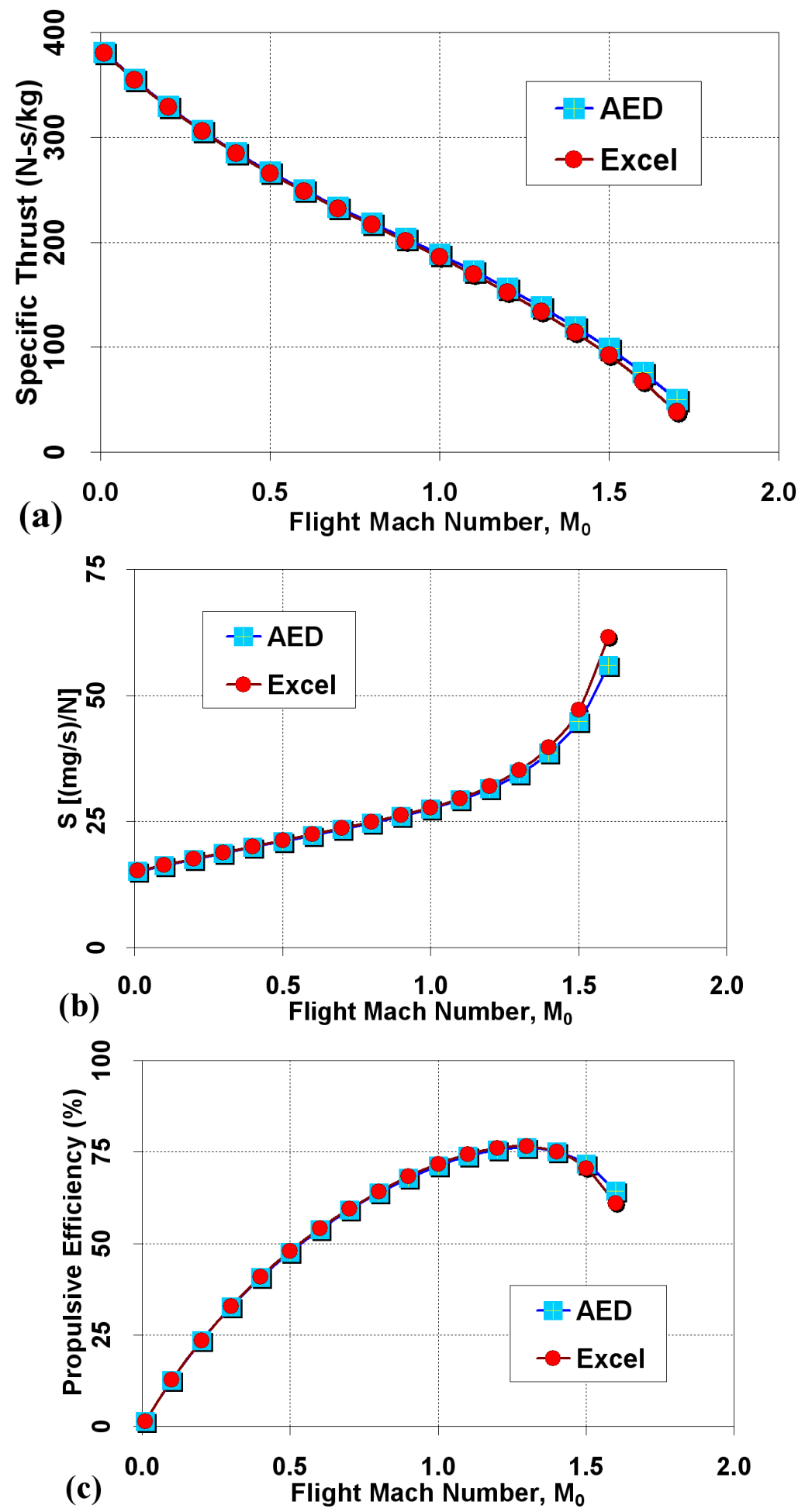

Figure 4.2: Comparison of the computed turbofan engine performance (without ITB) versus $M_{0}$ at an altitude of $10 \mathrm{~km}, \pi_{f}=2.0, \pi_{c}=28.48, \pi_{c L}=2.0, T_{t 4}=$ $1500 \mathrm{~K}$, and $\alpha=4.0$. 


\section{Chapter 5}

\section{Performance Cycle Analysis of a Turbofan Engine with ITB}

The performance of a selected reference point of a turbofan engine is desired at any flight conditions, throttle settings, and nozzle settings. A parametric cycle analysis has been performed for the reference-point engine using the method presented in previous chapter to give the so-called reference conditions (subscript R) for the engine $\left(F / \dot{m}_{0 R}, S_{R}\right.$ etc), for each engine component $\left(\pi_{f R}\right.$, $\tau_{f R}$, etc), and for the flight conditions $\left(M_{0 R}, P_{0 R}\right.$ and $\left.T_{0 R}\right)$.

To better understand the methodology of studying the performance cycle analysis problem, it is instructive to review how the parametric analysis proceeds again. For example, in the parametric cycle analysis problem as provided in Chapter 4, there are 11 independent equations for solving the values of the 11 dependent component parametric variables $\left(e . g ., \tau_{f}, \tau_{c L}, \tau_{c H}, f_{b}, \tau_{t H}, \pi_{t H}, \tau_{t L}\right.$, $\pi_{t L}, f_{i t b}, M_{9}$, and $\left.M_{19}\right)$. These 11 equations are solved for the given values of the independent quantities consisting of flight conditions (i.e., $M_{0}$ and $T_{0}$ ) and design choices (i.e., $\pi_{f}, \pi_{c L}, \pi_{c}$, and $\alpha$ ). With values of these 11 component variables in hand, the engine reference point performance in terms of $F / \dot{m}_{0}, S$, $\eta_{p}$, and $\eta_{t h}$ are readily found. 
Similarly, to find the off-design engine performance, the operational performance values of the 18 dependent variables listed in Table 5.1 must be determined and then, in turn, related by 18 independent equations to obtain a solution for each component performance variable. Since performance analysis is an indirect problem as opposed to the direct problem of parametric analysis, the solution of the 18 performance equations is not as straightforward as in the parametric case. Regardless of the difficulty in solving the equations, once the values of the 18 dependent variables in Table 5.1 are known, the engine performance in terms of $F / \dot{m}_{0}, S, \eta_{p}$, and $\eta_{t h}$ follows immediately. In the "Constant or Known" column of Table 5.1, $\pi_{b}, \pi_{i t b}, \pi_{n f}$, and $\pi_{n}$ are assumed to remain constant, and $\tau_{d}, \tau_{n}$, and $\tau_{n f}$ are assumed equal to one.

Table 5.1: Engine performance variables

\begin{tabular}{lccc}
\hline \hline Component & $\begin{array}{c}\text { Independent } \\
\text { Variable }\end{array}$ & $\begin{array}{c}\text { Constant } \\
\text { or Known }\end{array}$ & $\begin{array}{c}\text { Dependant } \\
\text { Variable }\end{array}$ \\
\hline Engine & $M_{0}, T_{0}, P_{0}$ & $\pi_{f}=f\left(M_{0}\right)$ & $\dot{m}_{0}, \alpha$ \\
Diffuser & & $\eta_{f}$ & $\pi_{f}, \tau_{f}$ \\
Fan & & $\eta_{c L}$ & $\pi_{c L}, \tau_{c L}$ \\
LPC & $T_{t 4}$ & $\eta_{c H}$ & $\pi_{c H}, \tau_{c H}$ \\
HPC & $T_{t 4.5}$ & $\pi_{b}$ & $f_{b}$ \\
Main Burner & $n$ & $\eta_{t H}, M_{4}$ & $\pi_{t H}, \tau_{t H}$ \\
HPT & & $\pi_{t .5}, M_{4.5}$ & $f_{i t b}$ \\
ITB & & $\pi_{f n}\left(\tau_{i t b}, n\right)$ & $\pi_{t L}, \tau_{t L}$ \\
LPT & $m$ & $\pi_{n}$ & $M_{18}, M_{19}$ \\
& & $A_{8}=f\left(\tau_{i t b}, m\right)$ & $M_{8}, M_{9}$ \\
Fan exhaust nozzle & 7 & & 18 \\
Core exhaust nozzle & & & \\
\hline Total number & & & \\
\hline \hline
\end{tabular}

\subsection{Assumptions}

In addition to the assumptions summarized in the parametric analysis in Chapter 4, the following assumptions are employed in the performance analysis: 
1. The flow area is constant at station 4, i.e., main burner exit or HPT entrance.

2. The flow is choked at the HPT entrance nozzles (station 4), at the LPT entrance nozzles (station 4.5), and at the throat of the exhaust nozzles (stations 8 and 18). Since the exhaust nozzles may unchoke at low throttle settings and influence the fan operating line, the cases of the unchoked exhaust nozzles (stations 8 and 18) are also included in this analysis.

3. The component efficiencies $\left(\eta_{f}, \eta_{c L}, \eta_{c H}, \eta_{t H}, \eta_{t L}, \eta_{b}, \eta_{i t b}, \eta_{m H}\right.$, and $\left.\eta_{m L}\right)$ are constant.

4. The total pressure ratios $\left(\pi_{d \max }, \pi_{b}, \pi_{i t b}, \pi_{n}\right.$, and $\left.\pi_{n f}\right)$ remains the same as the values in the parametric analysis.

5. The variation in fuel-air ratios $\left(f_{b}\right.$ and $\left.f_{i t b}\right)$ are ignored when compared to unity.

6. The exit areas $\left(A_{9}\right.$ and $\left.A_{19}\right)$ of the exhaust nozzles are adjustable so that the corresponding pressure ratios $\left(P_{9} / P_{0}\right.$ and $\left.P_{19} / P_{0}\right)$ can be set to predetermined values.

7. The area at each engine station is constant. However the areas of stations 4.5 and 8 change with the ITB setting to follow a specified function of $\tau_{i t b}$.

8. The diffuser total pressure ratio is given by $\pi_{d}=\pi_{d \max } \eta_{R s p e c}$ where $\pi_{d \max }$ is the total pressure ratio due to friction and $\eta_{R s p e c}$ is the total pressure recovery for the shocking system given by MIL-E-5008B [36].

\subsection{Modeling Approaches}

The station numbers and notations for the compressible flow remain the same as in the parametric analysis. Two techniques are frequently used in the 
analysis to follow. The first is called the referencing [34], and the second is the Mass Flow Parameter.

\subsubsection{Referencing}

The functional relations for the engine cycle analysis are based on the application of mass, energy, momentum, and entropy considerations to the onedimensional steady flow of a perfect gas at an engine reference or off-design steady state operating point. Thus, if, at any off-design point,

$$
f(\tau, \pi)=\text { constant }
$$

represents a relationship between the two performance variables $\tau$ and $\pi$ at a steady state operating point, then the constant can be evaluated at the reference point, so that

$$
f(\tau, \pi)=f\left(\tau_{R}, \pi_{R}\right)=\text { constant }
$$

since $f(\tau, \pi)$ applies to both on-design and off-design points. Basically, this technique replaces the constants with reference point values and prove to be very efficient in the performance analysis. For example, please refer to Eq.(5.10).

\subsubsection{Mass Flow Parameter}

The Mass Flow Parameter (MFP) is mainly based on the law of conservation of mass. It combines mass flow per unit area with the perfect gas law, Mach number, sound speed, and equations for total temperature and pressure. The resulting expression is

$$
M F P(M)=\frac{\dot{m} \sqrt{T_{t}}}{P_{t} A}=M \sqrt{\frac{\gamma}{R}\left(1+\frac{\gamma-1}{2} M^{2}\right)}
$$


When the other four quantities and the calorically perfect gas constants $(\gamma$ and $R$ ) are known at that station, the MFP may be used to find any single flow quantity. The MFP is often used, for example, to determine the flow area required to choke a given flow (i.e., at $M=1$ ). When the mass flow is conserved between them, the MFP becomes very important such that it develops valuable relationships between the flow properties at two different stations.

\subsection{Modeling of Engine Components}

In off-design analysis, it is necessary to predict the performance of individual component. There are two classes of predicting individual component performance [39]. First, the actual component characteristics can be obtained from the component hardware performance data, which give a better estimate. However, the actual component hardware is not yet available in this preliminary engine design phase. Instead, simple models of component behaviors are used in terms of their operating conditions.

\subsubsection{Turbine}

\section{High-pressure Turbine}

For our engine model, the mass flow entering the HPT equals that entering the main burner plus the fuel added in the main burner. Applying mass balance yields

$$
\dot{m}_{4}=\dot{m}_{3.1}+\dot{m}_{i t b}=\dot{m}_{c}\left(1-\beta-\epsilon_{1}-\epsilon_{2}\right)\left(1+f_{b}\right)
$$

Likewise, the mass flow entering the LPT equals that entering the HPT plus the fuel added in the ITB. Thus 


$$
\dot{m}_{4.5}=\dot{m}_{4.4}+\dot{m}_{i t b}=\dot{m}_{c}\left[\left(1-\beta-\epsilon_{1}-\epsilon_{2}\right)\left(1+f_{b}\right)+\epsilon_{1}+\epsilon_{2}\right]\left(1+f_{i t b}\right)
$$

Writing these using the MFP and the flow properties at stations 4 and 4.5 gives

$$
\dot{m}_{4}=\frac{P_{t 4}}{\sqrt{T_{t 4}}} A_{4} M F P\left(M_{4}\right)=\dot{m}_{c}\left(1-\beta-\epsilon_{1}-\epsilon_{2}\right)\left(1+f_{b}\right)
$$

and

$$
\dot{m}_{4.5}=\frac{P_{t 4.5}}{\sqrt{T_{t 4.5}}} A_{4.5} M F P\left(M_{4.5}\right)=\dot{m}_{c}\left[\left(1-\beta-\epsilon_{1}-\epsilon_{2}\right)\left(1+f_{b}\right)+\epsilon_{1}+\epsilon_{2}\right]\left(1+f_{i t b}\right.
$$

Solving each of these two expressions for $\dot{m}_{3}$ and equating them yields

$\frac{P_{t 4}}{\sqrt{T_{t 4}}} \frac{A_{4} M F P\left(M_{4}\right)}{\left(1-\beta-\epsilon_{1}-\epsilon_{2}\right)\left(1+f_{b}\right)}=\frac{P_{t 4.5}}{\sqrt{T_{t 4.5}}} \frac{A_{4.5} M F P\left(M_{4.5}\right)}{\left[\left(1-\beta-\epsilon_{1}-\epsilon_{2}\right)\left(1+f_{b}\right)+\epsilon_{1}+\epsilon_{2}\right]\left(1+f_{i t b}\right)}$

which can be rewritten in terms of ratios as follows

$\frac{P_{t 4.4}}{P_{t 4}} \frac{\sqrt{T_{t 4}}}{\sqrt{T_{t 4.5}}} A_{4.5}=A_{4} \frac{M F P\left(M_{4}\right)}{M F P\left(M_{4.5}\right)} \frac{\left[\left(1-\beta-\epsilon_{1}-\epsilon_{2}\right)\left(1+f_{b}\right)+\epsilon_{1}+\epsilon_{2}\right]\left(1+f_{i t b}\right)}{\left(1-\beta-\epsilon_{1}-\epsilon_{2}\right)\left(1+f_{b}\right)} \frac{P_{t 4.4}}{P_{t 4.5}}$

The right-hand side of the above equation is considered a constant since because of the following assumption: the flow is choked at stations 4 and 4.5, the flow areas at station 4 is constant, variations in fuel-air ratios $\left(f_{b}\right.$ and $\left.f_{i t b}\right)$ 
are ignored when compared to unity, and that the total pressure ratio of the ITB does not change. Using the referencing technique, we have

$$
\frac{P_{t 4.4}}{P_{t 4}} \frac{\sqrt{T_{t 4}}}{\sqrt{T_{t 4.5}}} A_{4.5}=\left(\frac{P_{t 4.4}}{P_{t 4}} \frac{\sqrt{T_{t 4}}}{\sqrt{T_{t 4.5}}} A_{4.5}\right)_{R}
$$

which can be rewritten in terms of component total pressure and total temperature ratios as

$$
\pi_{t H}=\frac{\sqrt{\tau_{t H} \tau_{i t b}}}{\sqrt{\tau_{t H R} \tau_{i t b R}}} \frac{A_{4.5 R}}{A_{4.5}} \pi_{t H R}
$$

This gives one relationship between the HPT's total pressure and total temperature ratios to meet mass conservation as a function of the ITB's total temperature ratio. A second equation between $\pi_{t H}$ and $\tau_{t H}$ comes from the HPT efficiency equation or

$$
\tau_{t H}=1-\eta_{t H}\left\{1-\pi_{t H}^{\left(\gamma_{t}-1\right) / \gamma_{t}}\right\}
$$

For reference values of $\pi_{t H}, \tau_{t H}, A_{4.5}$, and $\tau_{i t b}$, and the corresponding value of $\eta_{t H}$, Eqs. (5.11) and (5.12) yield the high-pressure turbine's operating point $\left(\tau_{t H}, \pi_{t H}\right)$ for a given value of $\tau_{i t b}$ and $A_{4.5}$ as sketched in Figure 5.1. The operating point is the intersection of Eqs. (5.11) and (5.12).

One relationship for $A_{4.5} / A_{4.5 R}$ is to assume that it is related to the total temperature ratio of the ITB $\left(\tau_{i t b}\right)$ raised to the power $n$ or

$$
\frac{A_{4.5}}{A_{4.5 R}}=\left(\frac{\tau_{i t b}}{\tau_{i t b R}}\right)^{n}
$$

For the case where $\frac{A_{4.5}}{A_{4.5 R}}=\sqrt{\frac{\tau_{i t b}}{\tau_{i t b R}}}$, then the solution to Eqs. 5.11) and 
(5.12) is $\pi_{t H}=\pi_{t H R}$ and $\tau_{t H}=\tau_{t H R}$.

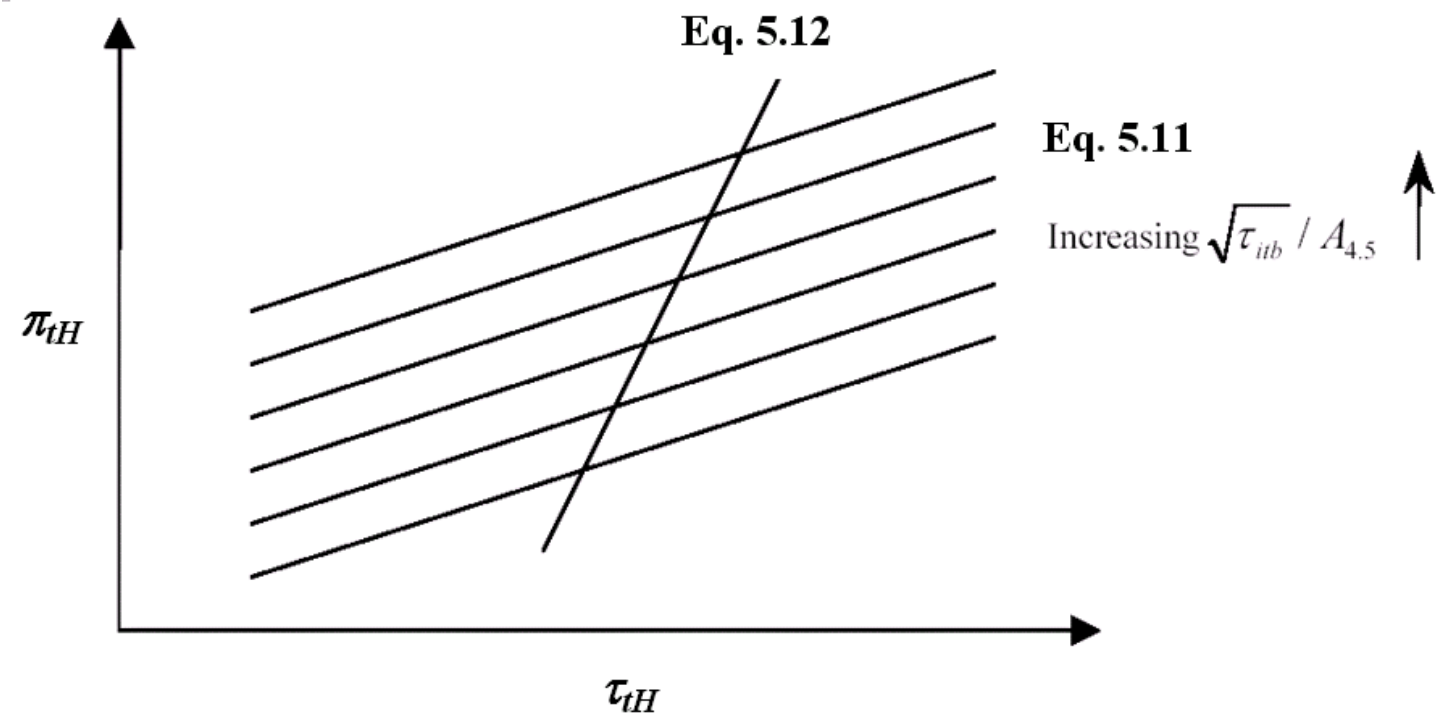

Figure 5.1: Variation in the HPT operating conditions with increasing $\sqrt{\tau_{i t b}} / A_{4.5}$

\section{Low-Pressure Turbine}

The mass flow rates at station 4.5 equals that at station 8 . Writing the mass conservation at stations 4.5 and 8 using the MFP and gives

$$
\frac{P_{t 4.5}}{\sqrt{T_{t 4.5}}} A_{4.5} M F P\left(M_{4.5}\right)=\frac{P_{t 8}}{\sqrt{T_{t 8}}} A_{8} \operatorname{MFP}\left(M_{8}\right)
$$

This can be rewritten as

$$
\frac{\pi_{t L}}{\sqrt{\tau_{t L}}}=\frac{A_{4.5}}{\pi_{n} A_{8}} \frac{\operatorname{MFP}\left(M_{4.5}\right)}{\operatorname{MFP}\left(M_{8}\right)}
$$

Noting that $\pi_{n}$ and $M_{4.5}(=1)$ are assumed constant, then the above equation can be rewritten using referencing technique as 


$$
\pi_{t L}=\pi_{t L R} \sqrt{\frac{\tau_{t L}}{\tau_{t L R}}} \frac{A_{8 R}}{A_{8}} \frac{A_{4.5}}{A_{4.5 R}} \frac{\operatorname{MFP}\left(M_{8 R}\right)}{\operatorname{MFP}\left(M_{8}\right)}
$$

This gives one relationship between the LPT's total pressure and total temperature ratios to meet mass conservation as a function of the core exhaust nozzle throat area $\left(A_{8} / A_{8 R}\right)$. A second equation between $\pi_{t L}$ and $\tau_{t L}$ comes from the LPT efficiency equation or

$$
\tau_{t L}=1-\eta_{t L}\left\{1-\pi_{t L}^{\left(\gamma_{i t b}-1\right) / \gamma_{i t b}}\right\}
$$

The operating condition $\left(\tau_{t L}, \pi_{t L}\right)$ of the LPT can be determined using Eqs. (5.16) and (5.17) along with the exhaust Mach number $M_{8}$ and specified functional relationships for the low pressure turbine nozzle throat area $\left(A_{4.5} / A_{4.5 R}\right)$ and the core exhaust nozzle throat area $\left(A_{8} / A_{8 R}\right)$. One relationship for $\left(A_{8} / A_{8 R}\right)$ is to assume that it is related to the total temperature ratio of the ITB $\tau_{i t b}$ raised to the power $m$ or

$$
\frac{A_{8}}{A_{8 R}}=\left(\frac{\tau_{i t b}}{\tau_{i t b R}}\right)^{m}
$$

With this functional relationship, the engine's LPT performance will vary the same as the turbofan without the ITB, when the ITB is turned off. For reference values of $\pi_{t L}, \tau_{t L}, \tau_{i t b}$, and the corresponding value of $\eta_{t L}$, Eq. (5.16) and (5.17) yield the LPT's operating point $\left(\tau_{t L}, \pi_{t L}\right)$ for a given value of $\frac{A_{8 R}}{A_{8}} \frac{A_{4.5}}{A_{4.5 R}} \frac{M F P\left(M_{8 R}\right)}{M F P\left(M_{8}\right)}$ as sketched in Figure 5.2. The operating point is the intersection of Eqs. 5.16) and (5.17). For the case where $\frac{A_{8}}{A_{8 R}}=\frac{A_{4.5}}{A_{4.5 R}}$ and $M_{8}=M_{8 R}$, then the solution to Eqs.5.16) and (5.17) is $\pi_{t L}=\pi_{t L R}$ and $\tau_{t L}=\tau_{t L R}$ 


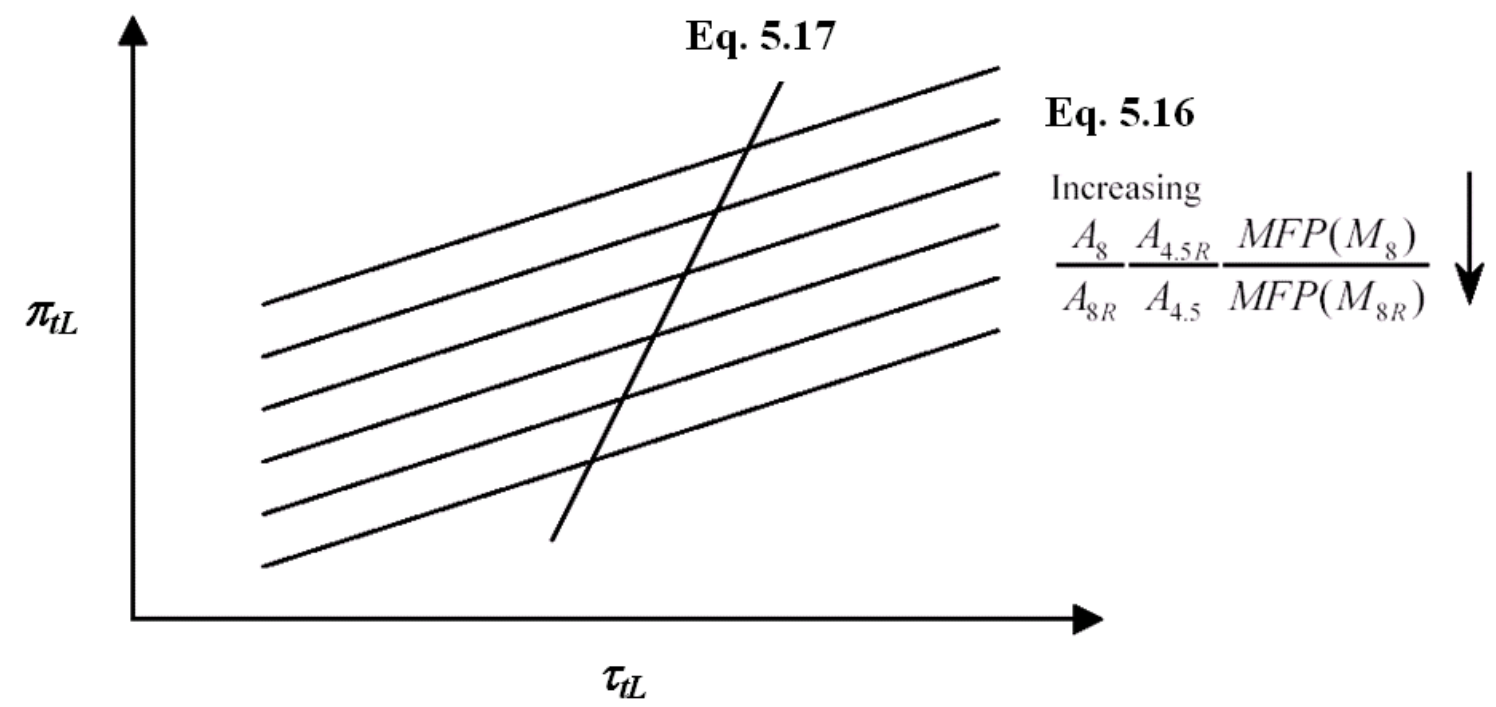

Figure 5.2: Variation in the LPT operating conditions with increasing $\frac{A_{8 R}}{A_{8}} \frac{A_{4.5}}{A_{4.5 R}} \frac{M F P\left(M_{8 R}\right)}{M F P\left(M_{8}\right)}$

\subsubsection{Fan Bypass Ratio}

An expression for the engine bypass ratio $(\alpha)$ follows directly from its definition written in terms of the properties at stations 4 and 18 .

$$
\alpha=\frac{\dot{m}_{f}}{\dot{m}_{c}}
$$

where

$$
\dot{m}_{f}=\frac{P_{t 18} A_{18}}{\sqrt{T_{t 18}}} \operatorname{MFP}\left(M_{18}\right)=\frac{P_{0} \pi_{r} \pi_{d} \pi_{f} \pi_{n f} A_{18}}{\sqrt{T_{t 13}}} \operatorname{MFP}\left(M_{18}\right)
$$

and

$$
\dot{m}_{4}=\dot{m}_{c}\left(1+f_{b}\right)=\frac{P_{t 4} A_{4}}{\sqrt{T_{t 4}}} M F P\left(M_{4}\right)=\frac{P_{0} \pi_{r} \pi_{d} \pi_{c L} \pi_{c H} \pi_{b} A_{4}}{\sqrt{T_{t 4}}} \operatorname{MFP}\left(M_{4}\right)
$$


Thus, the engine bypass ratio can be written as

$$
\alpha=\frac{\left(1+f_{b}\right)}{\pi_{c L} \pi_{c H} \pi_{b} /\left(\pi_{f} \pi_{n f}\right)} \sqrt{\frac{T_{t 4}}{T_{t 13}}} \frac{\operatorname{MFP}\left(M_{18}\right)}{\operatorname{MFP}\left(M_{4}\right)} \frac{A_{18}}{A_{4}}
$$

Since $\left(1+f_{b}\right), \pi_{b}, \pi_{n f}, A_{18}, A_{4}$, and $M_{4}(=1)$ are assumed constant and equal to their reference values, the bypass ratio can be rewritten using referencing technique as

$$
\alpha=\alpha_{R} \frac{\pi_{c L R} \pi_{c H R} / \pi_{f R}}{\pi_{c L} \pi_{c H} / \pi_{f}} \sqrt{\frac{T_{t 4} / T_{t 4 R}}{\tau_{r} \tau_{f} /\left(\tau_{r R} \tau_{f R}\right)}} \frac{M F P\left(M_{18}\right)}{\operatorname{MFP}\left(M_{18 R}\right)}
$$

\subsubsection{Fan and Low-pressure Compressor}

The performance equation for the total temperature ratio of the fan follows directly from the power balance of the low-pressure spool. Equating the power received by the fan and LPC to that delivered by the LPT gives

$$
\dot{m}_{f} c_{p c}\left(T_{t 13}-T_{t 2}\right)+\dot{m}_{c} c_{p c}\left(T_{t 2.5}-T_{t 2}\right)=\eta_{m L} \dot{m}_{4.5} c_{p i t b}\left(T_{t 4.5}-T_{t 5}\right)
$$

or

$$
\begin{aligned}
\dot{m}_{c} c_{p c} T_{t 2}\left[\alpha\left(\tau_{f}-1\right)+\left(\tau_{c L}-1\right)\right]= & \eta_{m L} \dot{m}_{c}\left[\left(1-\beta-\epsilon_{1}-\epsilon_{2}\right)\left(1+f_{b}\right)+\epsilon_{1}+\epsilon_{2}\right] \\
& \left(1+f_{i t b}\right) c_{p i t b} T_{t 4.5}\left(1-\tau_{t L}\right)
\end{aligned}
$$

Then dividing by $\dot{m}_{c} c_{p c} T_{t 2}$, gives

$$
\alpha\left(\tau_{f}-1\right)+\left(\tau_{c L}-1\right)=\eta_{m L}\left[\left(1-\beta-\epsilon_{1}-\epsilon_{2}\right)\left(1+f_{b}\right)+\epsilon_{1}+\epsilon_{2}\right]\left(1+f_{i t b}\right) \frac{\tau_{\lambda-i t b}}{\tau_{r}}\left(1-\tau_{t L}\right)
$$

Because the LPC and the fan are on the same shaft, it is reasonable to 
approximate that the total enthalpy rise of LPC is proportional to that of the fan. In other words, for a calorically perfect gas, the temperature rise of the LPC is therefore proportional to that of the fan [33; 34] and thus

$$
\frac{h_{t 2.5}-h_{t 2}}{h_{t 13}-h_{t 2}}=\frac{\tau_{c L}-1}{\tau_{f}-1}
$$

The use of referencing thus gives

$$
\frac{\tau_{c L}-1}{\tau_{f}-1}=\left(\frac{\tau_{c L}-1}{\tau_{f}-1}\right)_{R}
$$

Solving Eq.(5.27) for $\left(\tau_{c L}-1\right)$, substituting it into Eq.(5.26), and then solving for the fan total temperature ratio $\left(\tau_{f}\right)$, gives

$\tau_{f}=1+\left(\tau_{f R}-1\right) \eta_{m L} \frac{\tau_{\lambda-i t b}}{\tau_{r}}\left\{\frac{\left(1-\tau_{t L}\right)\left[\left(1-\beta-\epsilon_{1}-\epsilon_{2}\right)\left(1+f_{b}\right)+\epsilon_{1}+\epsilon_{2}\right]\left(1+f_{i t b}\right)}{\tau_{c L R}-1+\alpha\left(\tau_{f R}-1\right)}\right\}$

Using the fan efficiency, the fan total pressure ratio is given by

$$
\pi_{f}=\left\{1+\eta_{f}\left(\tau_{f}-1\right)\right\}^{\gamma_{c} /\left(\gamma_{c}-1\right)}
$$

The LPC total temperature ratio follows from Eq. (5.27) and thus

$$
\tau_{c L}=1+\left(\tau_{f}-1\right) \frac{\tau_{c L R}-1}{\tau_{f R}-1}
$$

Using the LPC efficiency, the LPC total pressure ratio is expressed as

$$
\pi_{c L}=\left\{1+\eta_{c L}\left(\tau_{c L}-1\right)\right\}^{\gamma_{c} /\left(\gamma_{c}-1\right)}
$$




\subsubsection{High-pressure Compressor}

The performance equation for the total temperature ratio of the HPC follows directly from the power balance of the high-pressure spool. Solving Eq. (4.24) for the total temperature ratio of the $\mathrm{HPC}\left(\tau_{c H}\right)$ gives

$$
\tau_{c H}=1+\eta_{m H}\left[\left(1-\beta-\epsilon_{1}-\epsilon_{2}\right)\left(1+f_{b}\right)+\epsilon_{1}\right] \frac{\tau_{\lambda-b}\left(1-\tau_{t H}\right)}{\tau_{r} \tau_{c L}}
$$

Using the HPC efficiency, the HPC total pressure ratio is given by

$$
\pi_{c H}=\left\{1+\eta_{c H}\left(\tau_{c H}-1\right)\right\}^{\gamma_{c} /\left(\gamma_{c}-1\right)}
$$

\subsubsection{Exhaust Nozzle}

The definitions for exhaust nozzles in the performance cycle analysis are similar to those in parametric cycle analysis. Thus, they share the same equations as clearly shown in Section 4.3 .8 .

The equation for the area ratio of the core exhaust nozzle follows from the mass conservation between stations 8 and 9, and the use of the MFP, giving

$$
\frac{A_{9}}{A_{8}}=\frac{1}{\pi_{n}} \frac{M F P\left(M_{8}\right)}{M F P\left(M_{9}\right)}
$$

On the other hand, the equation for the area ratio of the fan exhaust nozzle follows from the mass conservation between stations 18 and 19, and the use of the mass flow parameter, giving

$$
\frac{A_{19}}{A_{18}}=\frac{1}{\pi_{n f}} \frac{\operatorname{MFP}\left(M_{18}\right)}{\operatorname{MFP}\left(M_{19}\right)}
$$




\subsubsection{Engine Mass Flow Rate}

An expression for the overall engine mass flow rate $\left(\dot{m}_{0}\right)$ follows by the use of the mass flow parameter at station 4, giving

$$
\dot{m}_{0}=\dot{m}_{0 R} \frac{1+\alpha}{1+\alpha_{R}} \frac{P_{0} \pi_{r} \pi_{d} \pi_{c L} \pi_{c H}}{P_{0 R} \pi_{r R} \pi_{d R} \pi_{c L R} \pi_{c H R}} \sqrt{\frac{T_{t 4 R}}{T_{t 4}}}
$$

\subsubsection{Combustor and ITB}

The fuel-to-air ratios for the main burner $\left(f_{b}\right)$ and the ITB $\left(f_{i t b}\right)$ are determined by the user's choice. There are two models of the gas properties available in this analysis, i.e., Constant Specific Heat (CSH) model and the Modified Specific Heat $(M S H)$ model. Both models include the products of combustion in the estimation of heat capacity $\left(c_{p}\right)$. For the details, please refer to Appendix C.

\subsection{Computer Code}

Based on a similar code architecture with the parametric cycle code, the performance cycle code is written in combination between spreadsheet neuron cells, visual basic application, and Excel macro code. Again, this off-design cycle analysis program possesses all the features found in the on-design cycle code, one of which is that the code computes and plots the data sequentially without forcing users to open other types of plotting programs. An user's manual on how to use the program and its troubleshooting are included in the Appendix $\mathrm{H}$.

\subsubsection{Code Validation}

Due to the lack of the real engine data, it is useful to compare this result by the Excel code to those by the AEDsys program, also an integrated part of the AEDsys suite, which includes constraint analysis, aircraft system performance, mission analysis of aircraft system, and engine performance. [34] 
The performance of a conventional two-spool, separate-exhaust turbofan engine versus flight Mach number $\left(M_{0}\right)$ is computed using both AEDsys program and Excel code. As clearly shown in Figures 5.3 through 5.4 , the comparison of the full throttle performance was again found to be consistent. 

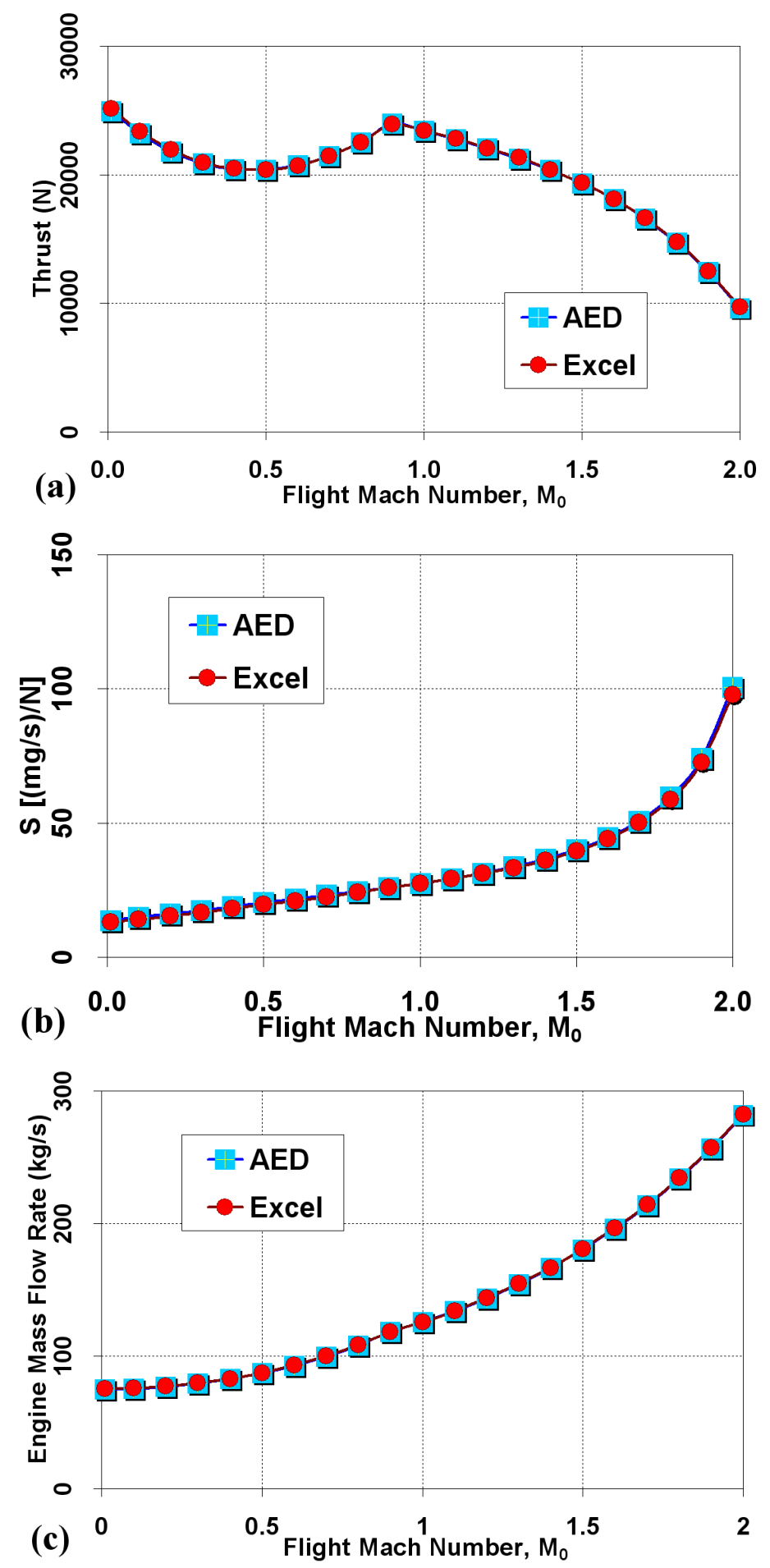

Figure 5.3: Comparison of the computed turbofan engine full throttle performance (without ITB) versus $M_{0}$ at an altitude of $10 \mathrm{~km}, M_{0 R}=0.9, \pi_{f R}=2.0$, $\pi_{c R}=28.48, \pi_{c L R}=2.0, T_{t 4 R}=1500 \mathrm{~K}, \alpha_{R}=4.0$, and $\dot{m}_{0 R}=118 \mathrm{~kg} / \mathrm{s}($ Part 1$)$. 

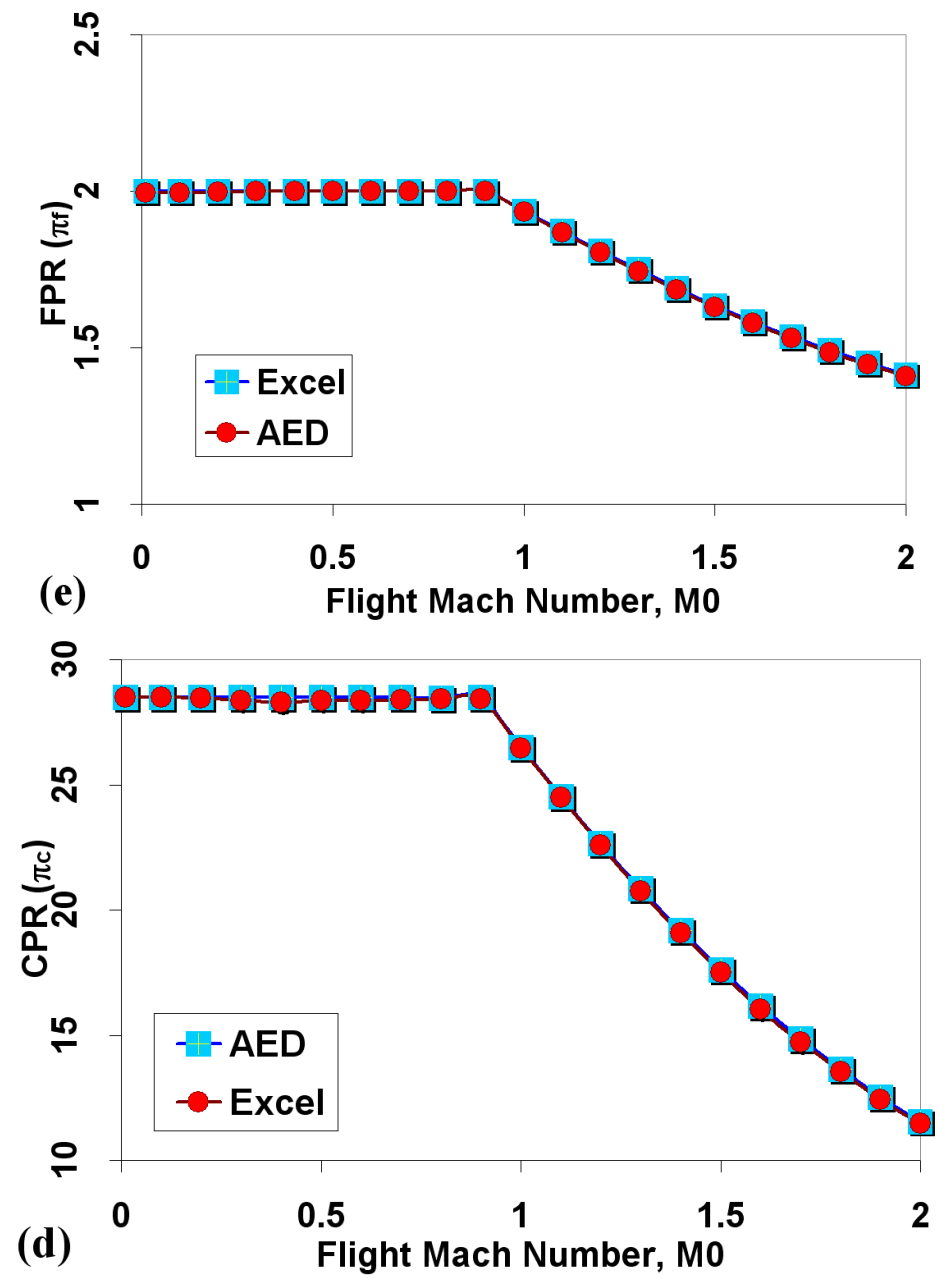

Figure 5.4: Comparison of the computed turbofan engine full throttle performance (without ITB) versus $M_{0}$ at an altitude of $10 \mathrm{~km}, M_{0 R}=0.9, \pi_{f R}=2.0$, $\pi_{c R}=28.48, \pi_{c L R}=2.0, T_{t 4 R}=1500 \mathrm{~K}, \alpha_{R}=4.0$, and $\dot{m}_{0 R}=118 \mathrm{~kg} / \mathrm{s}($ Part 2). 


\section{Chapter 6}

\section{Results and Discussions}

\subsection{Results from On-design Cycle analysis}

This section presents the results computed using the on-design Excel code. The validation of this code can be found in Section 4.5.1 while the user guideline is given in Appendix G.

First of all, the effect of turbine cooling on the engine performance will be investigated first followed by the variation of flight Mach number. Then, one design limitation (HPT inlet temperature) and three engine design choices, namely (1) CPR, (2) FPR, and (3) FBR, are studied in their subsonic $\left(M_{0}>1\right)$ and supersonic $\left(M_{0}>1\right)$ flight regimes, respectively. All these design parameters are then used to obtain the system performance parameters of ST, S, $\eta_{t h}, \eta_{p}$ and $\eta_{\circ}$.

\section{Engine Configuration}

Engine configurations used in these computations include a baseline engine (i.e., a conventional turbofan engine without an ITB) and ITB engines with different values of LPT inlet temperature (i.e., $T_{t 4.5}$ or ITB exit temperature). The 
maximum value of $T_{t 4.5}$ is $1300 \mathrm{~K}$ so that no cooling air is required for the LPT. Meanwhile, the engine specifications for the simulation of each design choices are summarized in Appendix J.

Other design parameters, such as component efficiencies, combustor efficiencies, mechanical efficiencies, and pressure drop/increase across various components, are user-defined input parameters. These design parameters are identical for each computation in this section and is listed in Table J.1.

\subsubsection{Effect of Turbine Cooling}

Figure 6.1 shows the performance comparison for the base turbofan engine with and without turbine cooling at flight Mach number of 0.0 to 2.0. Specific thrust (ST) of the uncooled base engine is overestimated by around 16 percent at subsonic flight $\left(M_{0}=0.9\right)$ and 28 percent at supersonic flight $\left(M_{0}=1.5\right)$. Therefore, a turbine-cooling model is required to account for the effect of turbine cooling on the engine performance.

\subsubsection{Effect of Flight Mach Number}

\section{High FBR}

Figures 6.2 a through 6.2 demonstrate the performance comparisons between a baseline turbofan engine and three different ITB engines of high FBR at flight Mach number of 0.0 to 2.0. For each engine, the compressor pressure ratio $(\mathrm{CPR})$, fan pressure ratio $(\mathrm{FPR})$, and $\mathrm{FBR}$ are fixed at 28.48, 1.3, and 4.0 , respectively, with a maximum allowable $T_{t 4}$ of $1600 \mathrm{~K}$. All types of engines exhibit a decrease in ST and an increase in S as flight Mach number increases.

Clearly, the ITB engine with $T_{t 4.5}$ of $1300 \mathrm{~K}$ (the highest $T_{t 4.5}$ among all other ITB engines) performs better than the baseline engine at both subsonic and supersonic flights. There is a rise in both ST and thermal efficiency without any increase in S. Nevertheless, the overall efficiency is not improved much due 

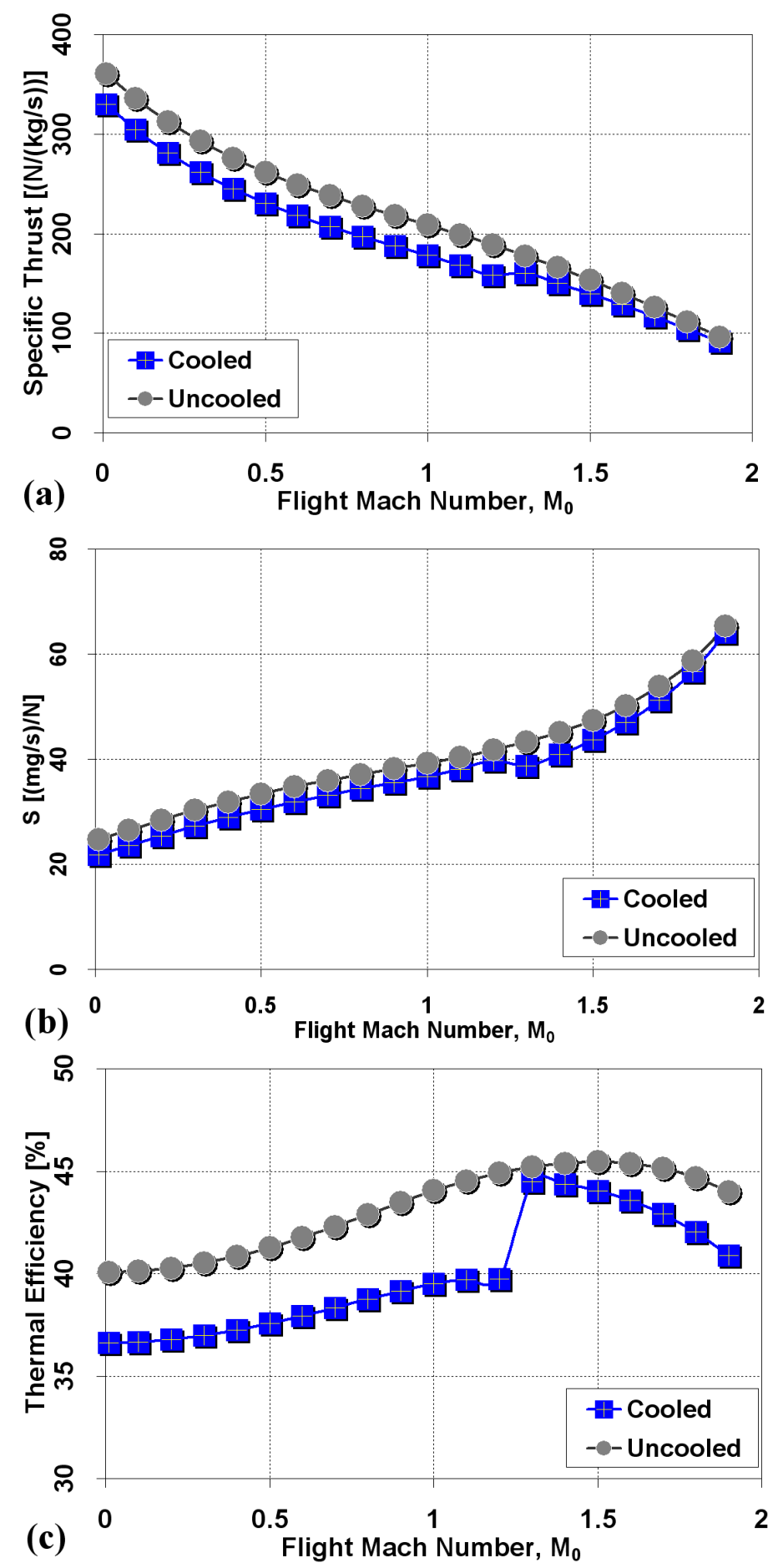


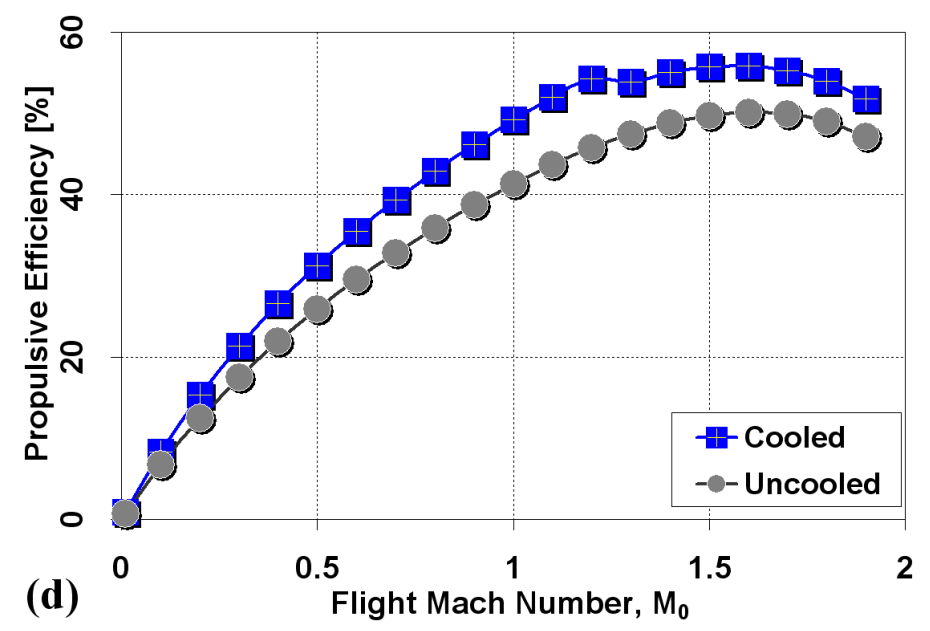

Figure 6.1: Performance comparison of turbofan engines versus $M_{0}$ at an altitude of $10 \mathrm{~km}, \pi_{f}=1.3, \pi_{c}=28.48, T_{t 4}=1800 \mathrm{~K}$, and $\alpha=4.0$.

to the decrease in the propulsive efficiency.

ITB engines with lower values of $T_{t 4.5}$ (i.e., $1150 \mathrm{~K}$ ) are beneficial at supersonic flight only (e.g., $M_{0}>1.3$ in this case). On the contrary, an ITB engine with the lowest value of $T_{t 4.5}$ (i.e., $1000 \mathrm{~K}$ ) gains no improvement at all. One reason is that this ITB engine is operating at $T_{t 4}$ (i.e., $1600 \mathrm{~K}$ ) greater than the $T_{c i}$ (i.e., $1319 \mathrm{~K}$ at $M_{0}$ of 0.85 ). (Note: Please refer to section 6.1.3 for the definition of $T_{c i}$.) The effect of flight Mach number on $T_{c i}$ is considered negligible because $T_{c i}$ only increases slightly with increasing flight Mach number (data not shown here).

One may argue to use a higher value for $T_{t 4.5}$. However, at higher $T_{t 4.5}$, especially when it is greater than the blade material limit, turbine cooling is required for LPT. This is unfavorable because more air would be extracted from LPC to cool down LPT, which may offset any advantage of having higher $T_{t 4.5}$. Therefore, in order to avoid cooling LPT, the maximum $T_{t 4.5}$ will be the turbine blade material limit (i.e., $1300 \mathrm{~K}$ in this study).

While having a better thermal efficiency than the baseline engine, ITB engine seems to suffer a decrease in propulsive efficiency. One can explain this by referring to equation 4.57) for the power out of the engine and equation 4.62 
for the propulsive efficiency. For each ITB engine, data showed that the rate of increase of the propulsive power (i.e., the numerator of the $\eta_{P}$ equation) is lower than the rate of increase of the kinetic energy gain out of the engine (i.e., the denominator of the $\eta_{P}$ equation), leading to a decreasing trend of propulsive efficiency. Further investigation shows that the cause of this phenomenon is due to the very high core exhaust velocity, which is not efficiently transferred into useful propulsive force in the first place. Most of the energy is wasted as jet kinetic energy. Meanwhile, according to Vogeler [53], he found that most of the gas expansion already took place in the HPT, thus the low pressure ratio across LPT did not allow an effective use of the additional energy from ITB in the fan.

\section{Low FBR}

The performance comparisons between the baseline turbofan engine and three different ITB engines of low FBR are shown in figures 6.3 through 6.3 f. For each engine, the CPR, FPR, and FBR are fixed at 28.48, 2.5, and 0.5, respectively.

Clearly, all three low-FBR ITB engines yields similar performance trends as the high-FBR ITB engines. As for the ITB engine with $T_{4.5}$ of $1300 \mathrm{~K}$, it has a better specific fuel consumption (e.g., average 3\%) than the baseline engine as well as the high-FBR ITB engine (e.g., average 1\%). This gives ITB better performance at supersonic flight than at subsonic flight. 

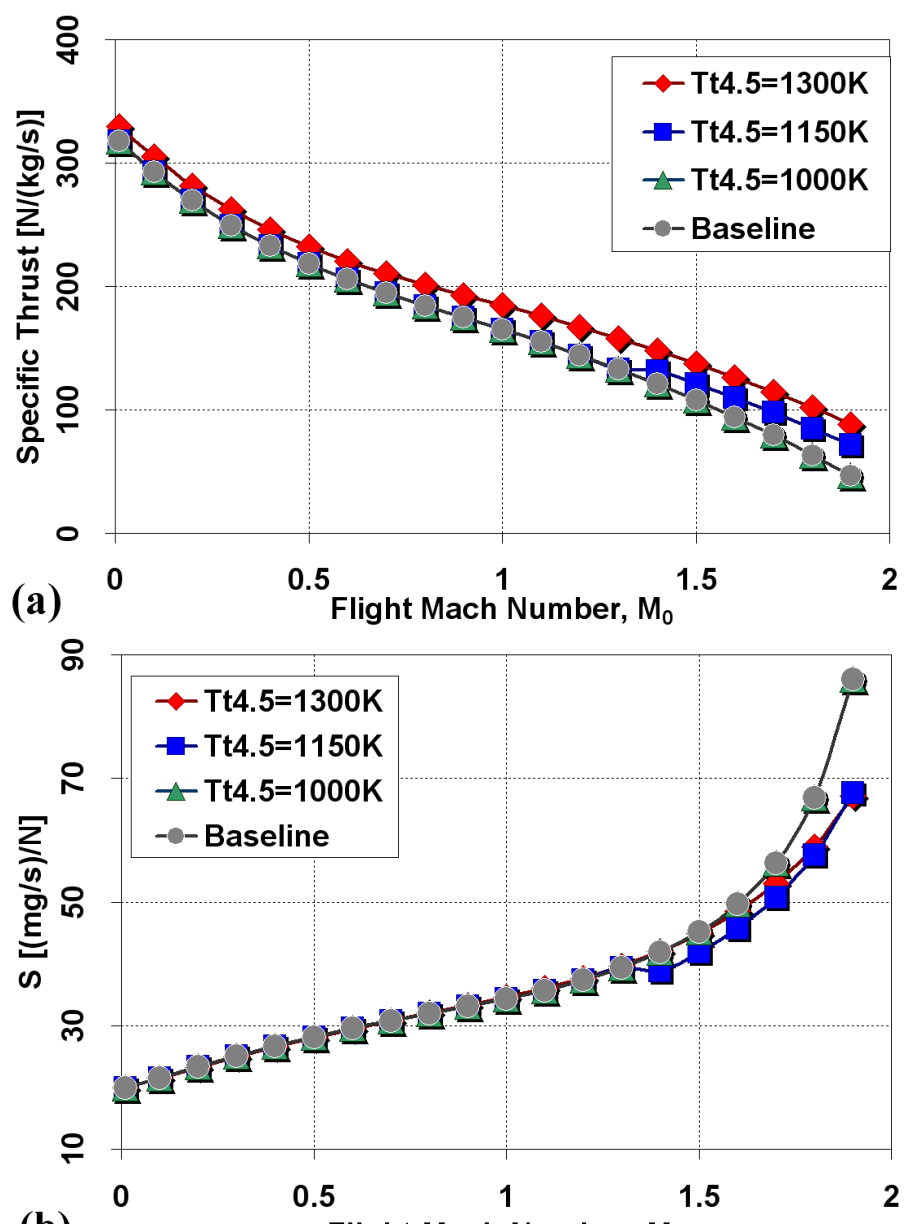

(b)

Flight Mach Number, $M_{0}$

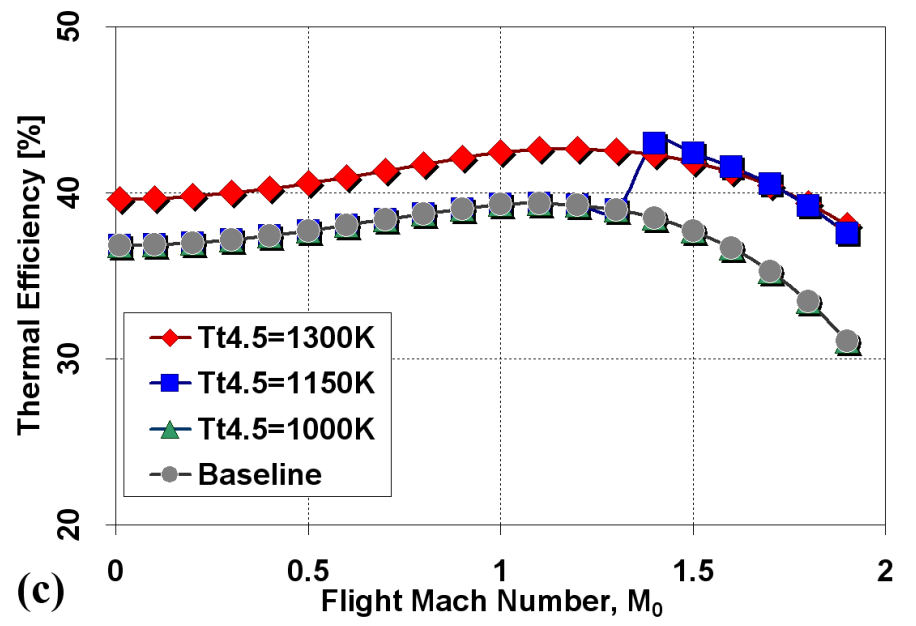



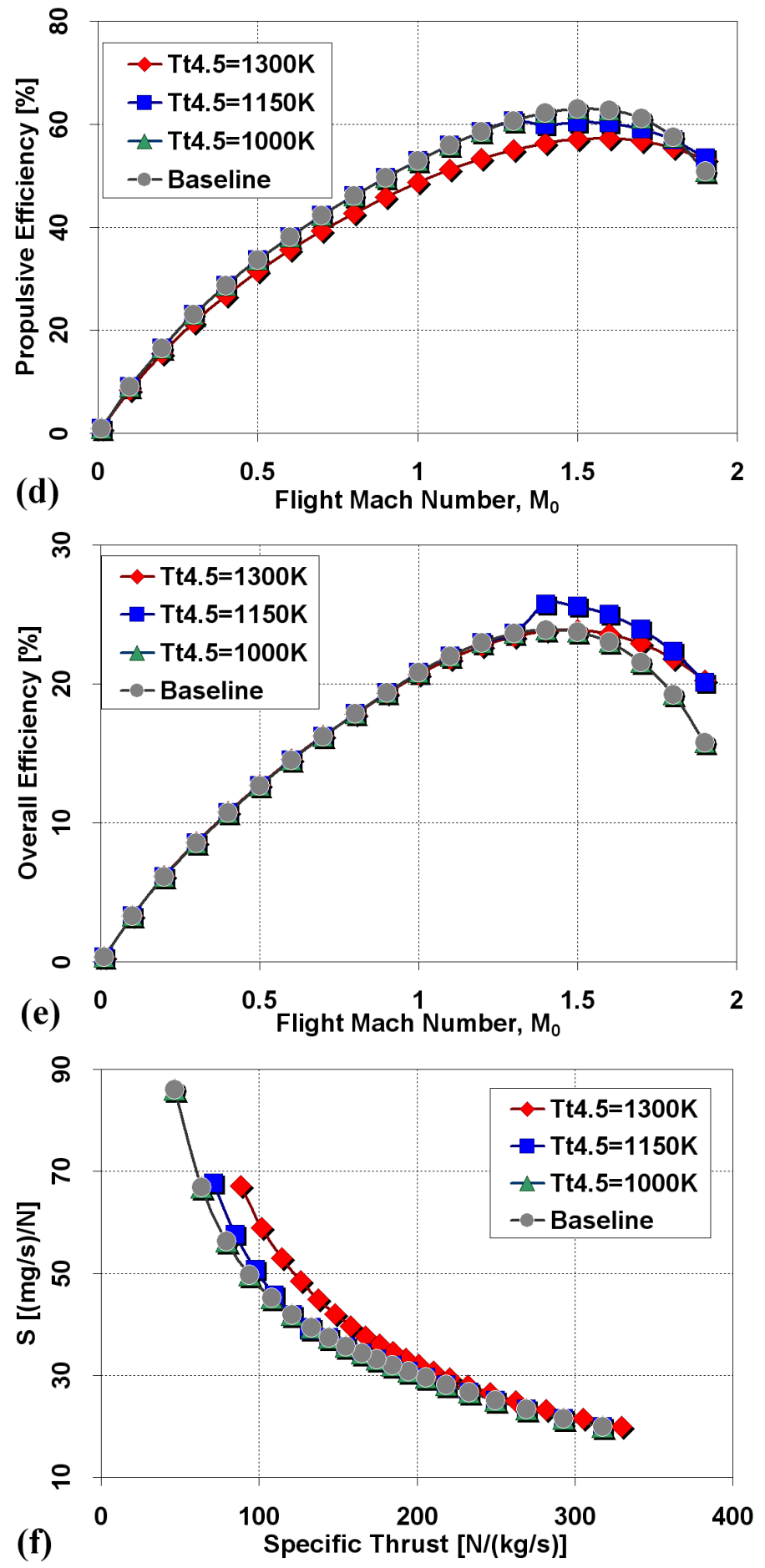

Figure 6.2: Performance comparison of turbofan engines versus $M_{0}$ at an altitude of $10 \mathrm{~km}, T_{t 4}=1600 \mathrm{~K}, \pi_{f}=1.3, \pi_{c}=28.48, \pi_{c L}=1.387$, and $\alpha=4.0$. 


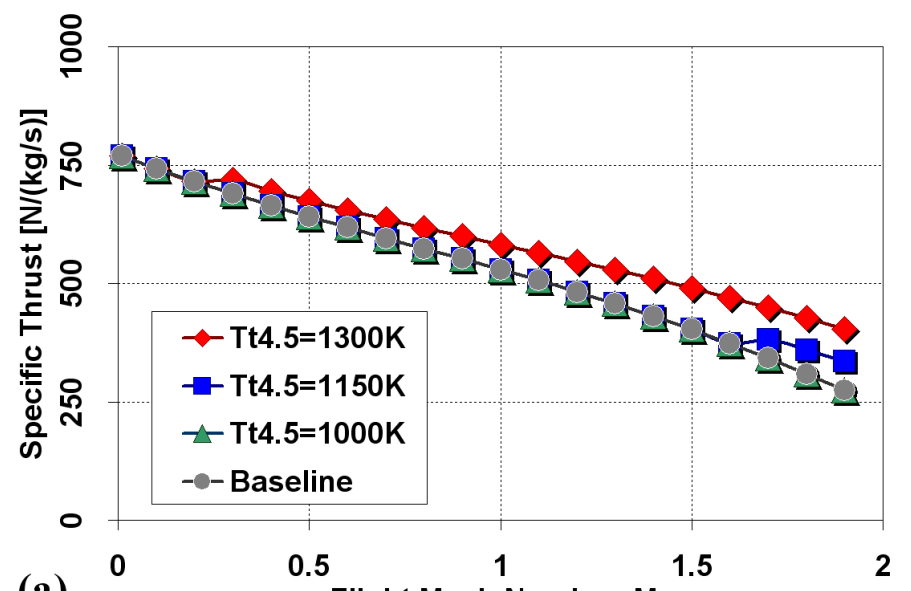

(a)

Flight Mach Number, $M_{0}$

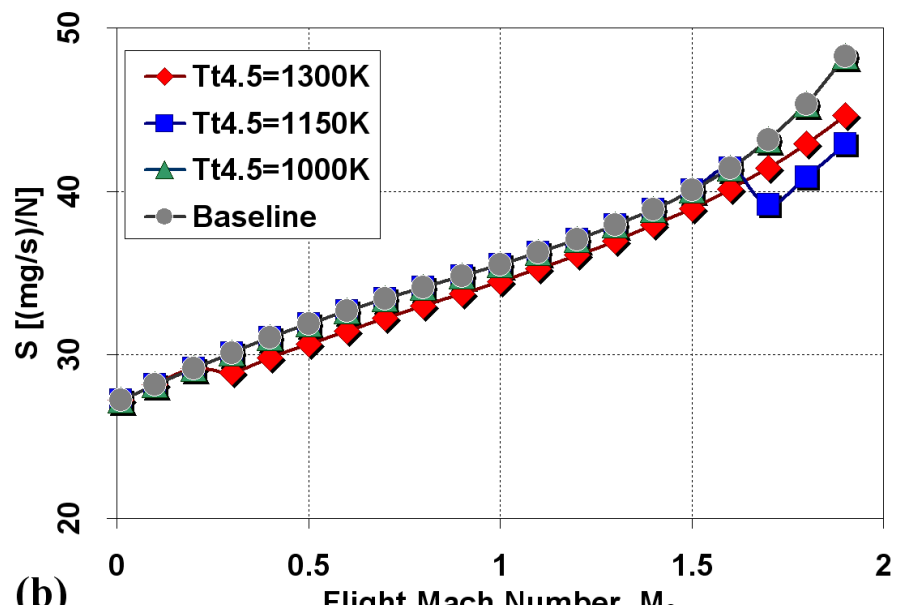

(b) Flight Mach Number, $M_{0}$

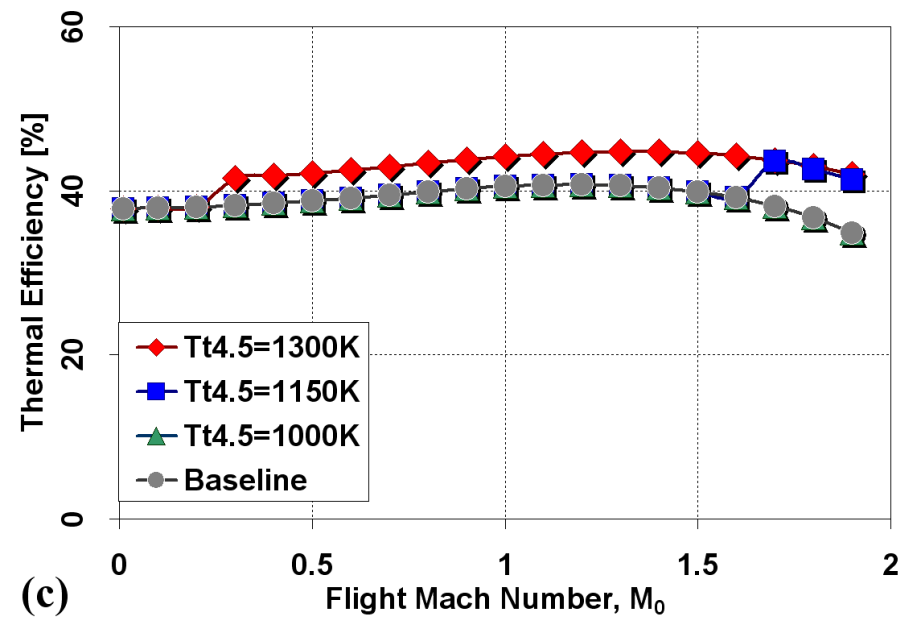



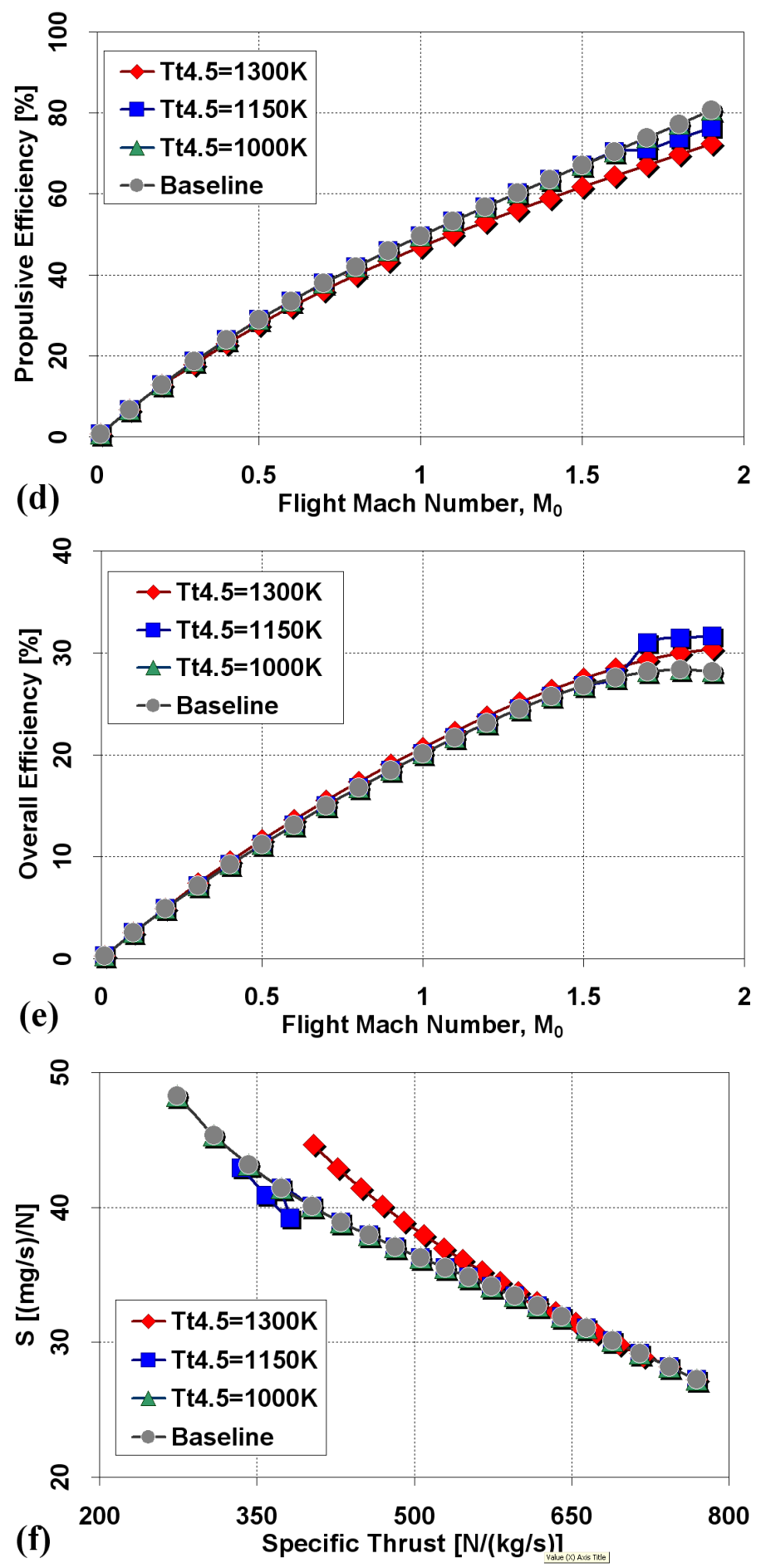

Figure 6.3: Performance comparison of turbofan engines versus $M_{0}$ at an altitude of $10 \mathrm{~km}, T_{t 4}=1600 \mathrm{~K}, \pi_{f}=2.5, \pi_{c}=28.48, \pi_{c L}=1.387$, and $\alpha=0.5$. 


\subsubsection{Effect of HPT Inlet Temperature}

\section{Subsonic Flight}

Figures 6.4 through 6.4 compare the engine performances of the baseline engine with three different ITB engines for varying HPT inlet temperatures $\left(T_{t 4}\right)$ from $1000 \mathrm{~K}$ to $2000 \mathrm{~K}$ at $M_{0}$ of 0.85 (i.e., subsonic flight). For each engine, CPR, FPR, and FBR are fixed at 28.48, 1.3, and 4.0, respectively. In Liu and Sirignano [32, it is clearly shown that the TB engines benefits from higher $T_{t 4}$ at both subsonic and supersonic flights. Similarly, in this case, as the value of $T_{t 4}$ increases, all ITB engines exhibit an increase in both ST and thermal efficiency, accompanied by a decrease in $\mathrm{S}$.

Data shows that whenever the gain in ST is greater than the loss due to extra fuel consumption in ITB, it leads to a better thermal efficiency. Clearly, ITB engines always generate higher ST and gain better thermal efficiency than the baseline engine. Nevertheless, this is not the case when $T_{t 4}$ is lower than $T_{t 4.5}$, in which a large portion of fuel is now burned in the ITB (i.e., a relatively lower pressure environment compared to the main burner); therefore, thermal efficiency will drop and be equivalent to that of the baseline engine. The thermal efficiency curves in the region of lower HPT inlet temperature (i.e., $T_{t 4}$ lower than $1300 \mathrm{~K}$ ) clearly reflect this phenomenon.

\section{Critical ITB Temperature $\left(T_{c i}\right)$}

However, it is also noticed that for all ITB engines, each ST and thermal efficiency curve will drop and merge with the base engine curve after reaching a maximum point at some values of $T_{t 4}$. These maximum points in the $\mathrm{ST}$ curves and thermal efficiency curves correspond to the minimum points in $\mathrm{S}$ curves. We refer to these values of $T_{t 4}$ as "critical ITB temperatures" $\left(T_{c i}\right)$, beyond which the ITB will be turned off, resulting in a discontinuity on each performance curve. 

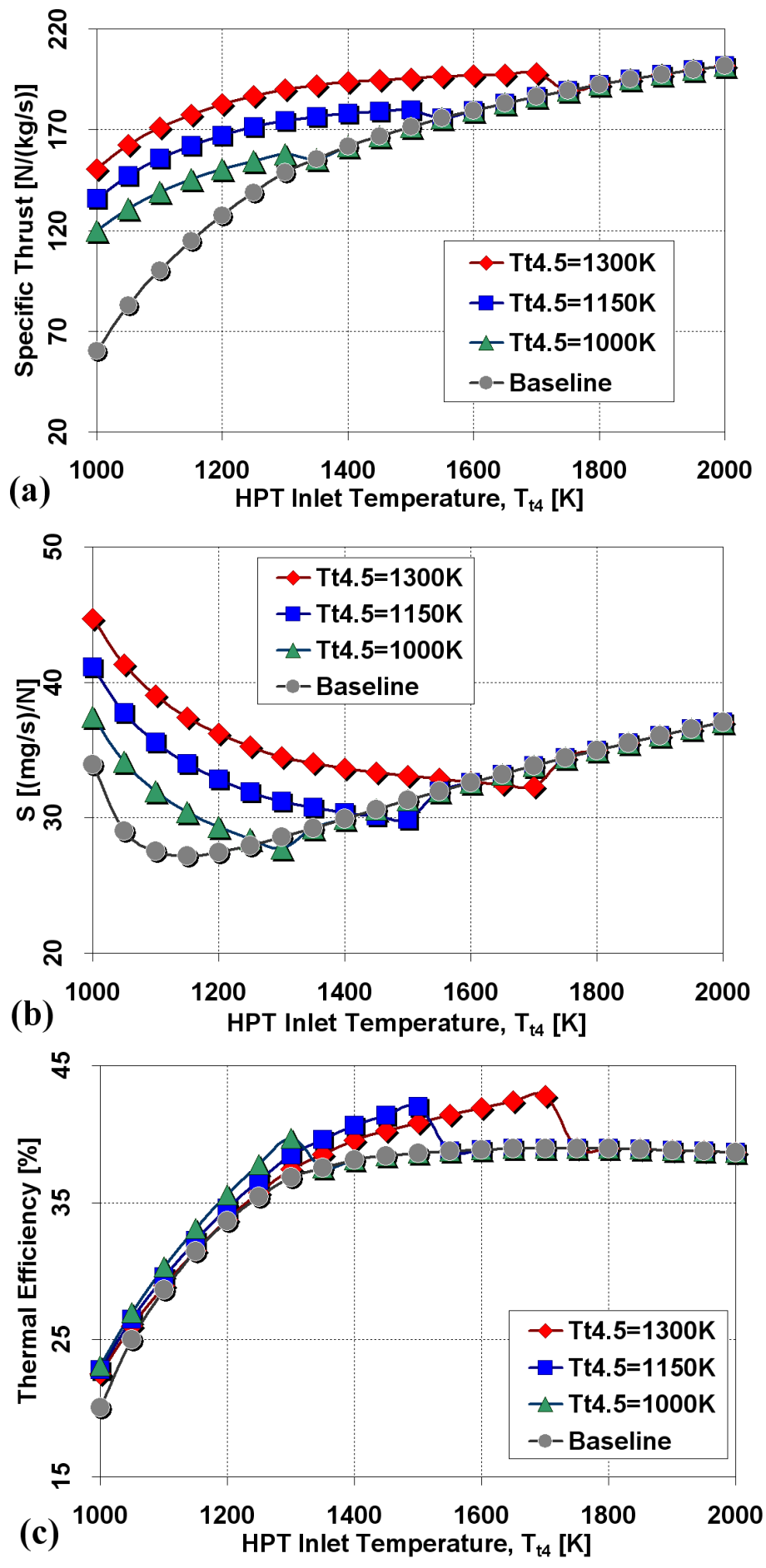

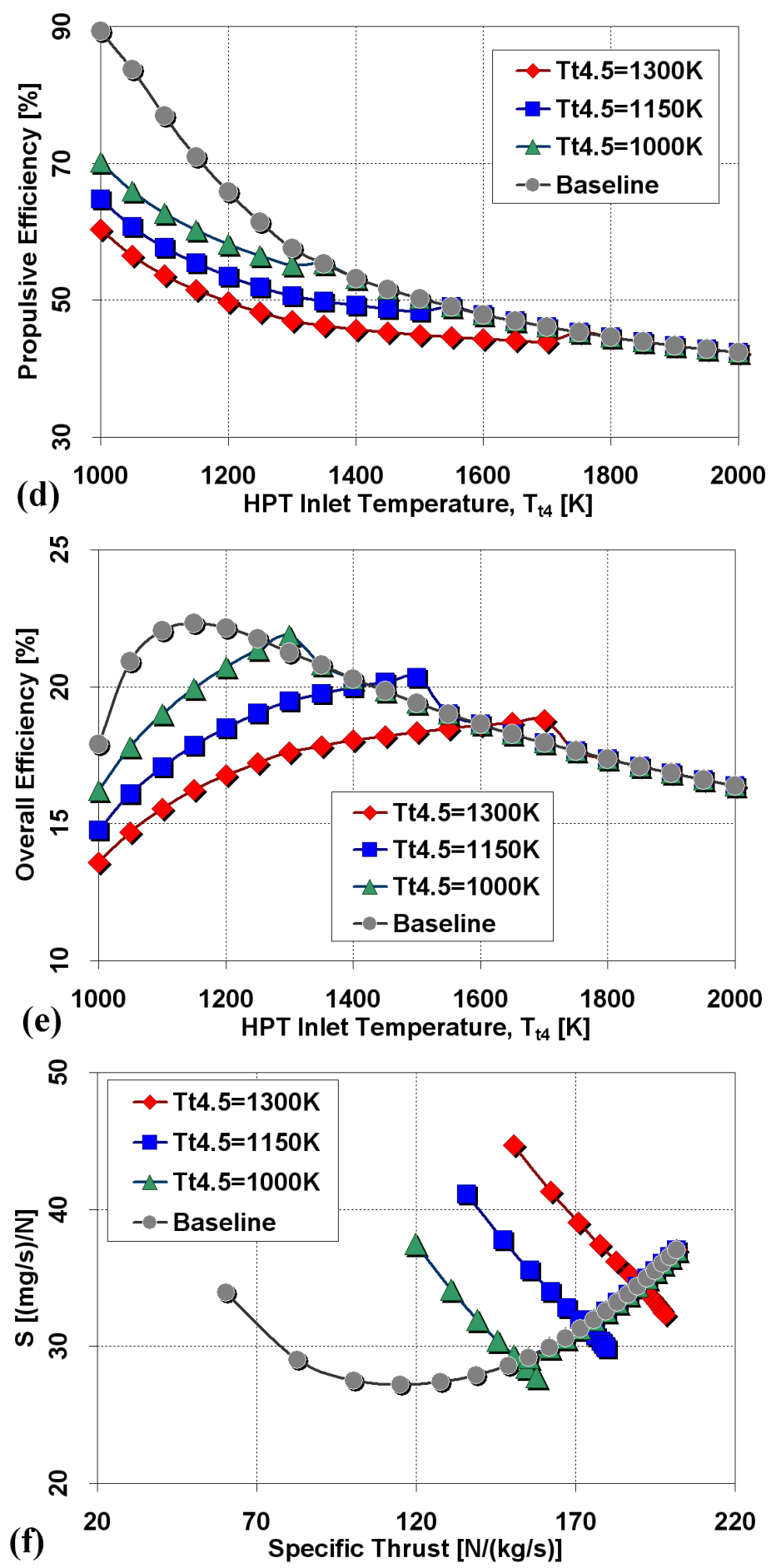

Figure 6.4: Performance comparison of turbofan engines versus $T_{t 4}$ at an altitude of $10 \mathrm{~km}, M_{0}=0.85, \pi_{f}=1.3, \pi_{c}=28.48, \pi_{c L}=1.387$, and $\alpha=4.0$. 
One can explain this by looking at Eq. 4.16 for the computation of the ITB fuel-air ratio. It is possible to yield a negative value of $f_{i t b}$ when the total enthalpy ratio after the expansion across $\operatorname{HPT}\left(\tau_{\lambda-b} \tau_{m 1} \tau_{m 2} \tau_{t H}\right)$ is greater than the ITB exit total enthalpy ratio $\left(\tau_{\lambda-i t b}\right)$ as shown below.

$$
\tau_{\lambda-i t b}-\tau_{\lambda-b} \tau_{m 1} \tau_{m 2} \tau_{t H}<0
$$

At such a condition, no further energy addition is allowed in ITB. Even worse, a negative value of $f_{i t b}$ means that energy needs to be extracted from ITB, which is considered a counteraction of adding ITB, and it is not desirable. Unfortunately, this also tells us that the ITB engines at $T_{t 4}$ higher than $T_{c i}$ will not benefit from the advantages of ITB, unless $T_{t 4.5}$ is further increased. For instance, a base engine with $T_{t 4}$ of $1600 \mathrm{~K}$ will only benefit from adding ITB if $T_{t 4.5}$ is set to $1300 \mathrm{~K}$ (see Figure 6.4 because its corresponding value of $T_{c i}$ (i.e., $1713 \mathrm{~K}$ as shown in Figure 6.5) is greater than $T_{t 4}$ of $1600 \mathrm{~K}$. Therefore, it is always desirable to seek a higher $T_{c i}$ in the engine design process in order to utilize the advantage of ITB. An example is in Liu and Sirignano's study [48], where $T_{06}$ (equivalent to $T_{t 4.5}$ in this paper) is specified at $1900 \mathrm{~K}$, much higher than $T_{04}$ (equivalent to $T_{t 4}$ ) of $1500 \mathrm{~K}$. This explains why $T_{c i}$ is never seen in their analysis. As mentioned previously, one concern of turbine cooling is the reduction in engine performance. Having a high value of $T_{t 4.5}$ (i.e., $1900 \mathrm{~K}$ ), turbine cooling is also required for the LPT, which may offset the advantage of using ITB.

To predict $T_{c i}$, the Eq. 6.1) is rewritten as shown in Eq. 6.2 below.

$$
\tau_{\lambda-b} \tau_{m 1} \tau_{m 2} \tau_{t H}=\tau_{\lambda-i t b}
$$

where $\tau_{m 1}$ and $\tau_{m 2}$ are mainly functions of $\tau_{\lambda-b}$.

The value of $T_{t 4}$ that satisfies Eq. 6.2) is referred as $T_{c i}$. Eq. (6.2) is solved by an iterative procedure and the result is shown in Figure 6.5. It is found that 
the higher the value of $T_{t 4.5}$, the higher the value of $T_{c i}$. This is because a higher $T_{t 4.5}$ allows more heat addition in ITB.

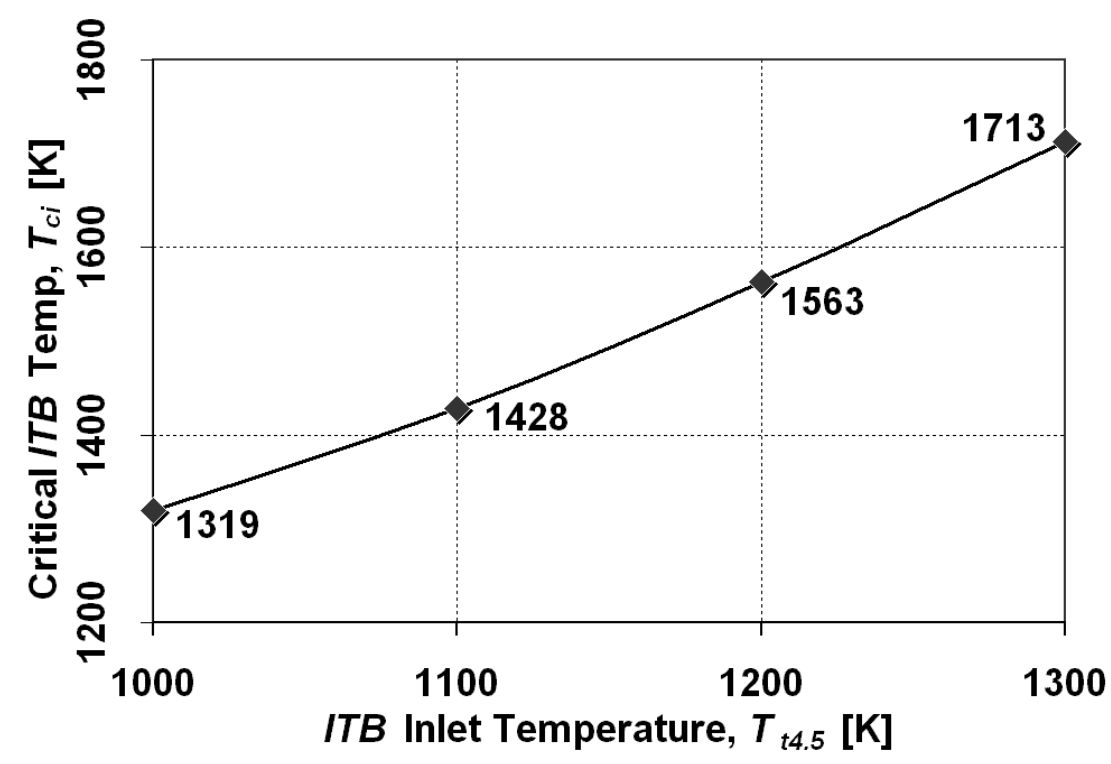

Figure 6.5: Variation of critical ITB temperature $\left(T_{c i}\right)$ with $T_{t 4.5}$ at $M_{0}=0.85$, $\pi_{f}=1.3, \pi_{c}=28.48$, and $\alpha=4.0$.

\section{Supersonic Flight}

At the supersonic flight, the CPR, FPR, and FBR of each engine are fixed at $28.48,3.0$, and 0.5 , respectively. The performance comparisons between the baseline turbofan engine and three different ITB engines at $M_{0}$ of 1.2 are shown in figures 6.6 a through 6.6.

Comparing Figure 6.4 to figure 6.6, it is clear that ITB engines at both subsonic and supersonic flight regimes have a very similar performance trends. Despite the similarity, ITB engine at supersonic flight has a better thermal efficiency (e.g, about $10 \%$ gain in each case) than at subsonic flight (e.g., up to $7 \%$ gain) because the fuel is burned in a higher pressure environment due to the ram effect at the engine inlet.

Unfortunately, at both subsonic and supersonic flights, the ITB engine is 
only better only when the engine operates at a range of $T_{t 4}$ close to the value of $T_{c i}$. According to figures 6.4 and 6.6 , the overall efficiencies are better at the $T_{t 4}$ range close to $T_{c i}$.

\subsubsection{Effect of Compressor Pressure Ratio}

\section{Subsonic Flight}

Figures 6.7 through 6.7f compare the engine performances of the baseline engine with three different ITB engines for varying CPR at a subsonic speed $\left(M_{0}=0.85\right)$ with $T_{t 4}$ of $1600 \mathrm{~K}$. The FPR and FBR are fixed at 1.3 and 4.0, respectively. As CPR increases, the baseline engine exhibits a decrease in ST

and $\mathrm{S}$ with an almost constant level of thermal efficiency. The ITB engine with $T_{t 4.5}$ of $1000 \mathrm{~K}$ behaves just like a baseline engine because $T_{t 4}$ exceeds $T_{c i}$, where ITB is turned off.

One would expect to see that ITB engine with $T_{4.5}$ of $1300 \mathrm{~K}$ gains an improvement in ST and thermal efficiency. However, it does not perform as well as one may thought, especially at the CPR higher than 30. As the CPR goes beyond 30, the decreasing propulsive efficiency has counteracted the overall performance of the ITB engine. On the other hand, ITB engine with a moderate value of $T_{t 4.5}$, i.e., $1150 \mathrm{~K}$ in this case, performs surprisingly well at CPR higher than 40 and above. These facts tell us that a higher value of $T_{t 4.5}$ does not necessarily mean that the engine will perform better.

\section{Supersonic Flight}

Holding all parameters constant, now consider the military turbofan engines with a higher FPR of 3.0 (multi-stage fan) and a low FBR of 0.5. Figure 6.8 a through 6.8 shows the performance comparison for these types of engines at $M_{0}$ $=1.2$. Because of the similar trends of performance, most of what have been observed and discussed in the subsonic flight conditions can be equally applied 

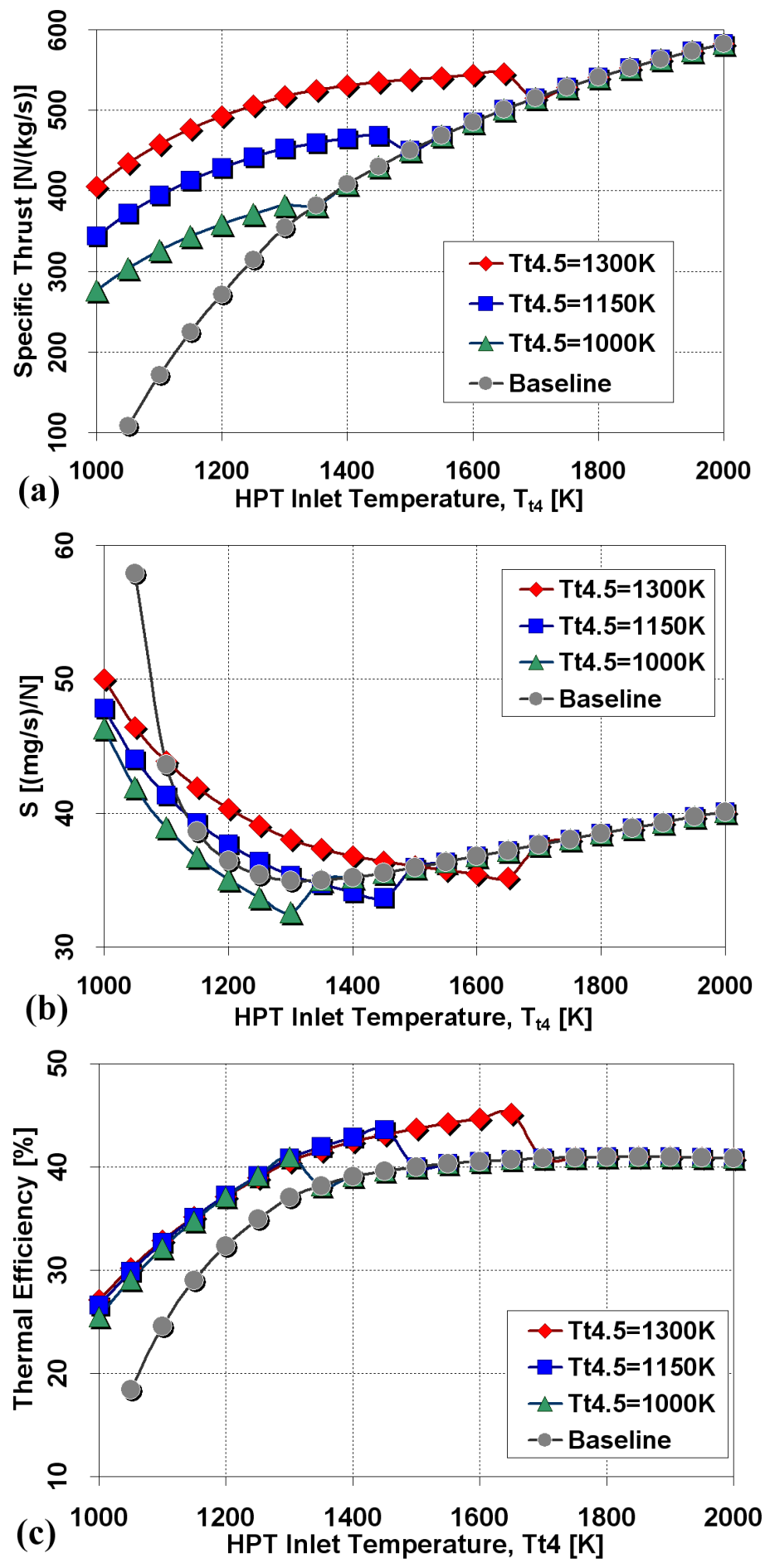

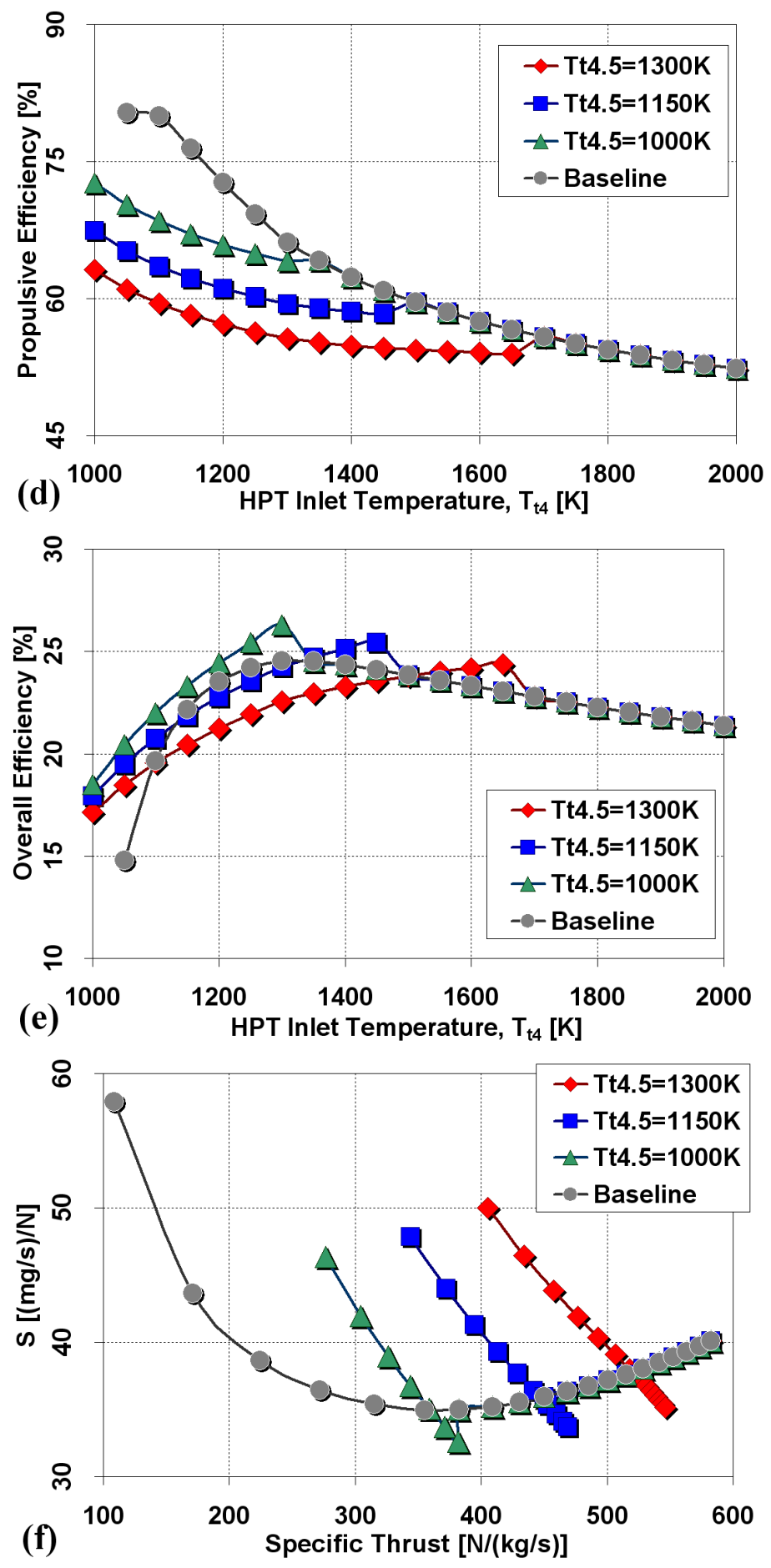

Figure 6.6: Performance comparison of turbofan engines versus $T_{t 4}$ at an altitude of $10 \mathrm{~km}, M_{0}$ of $1.2, \pi_{f}=3.0, \pi_{c}=28.48, \pi_{c L}=1.387$, and $\alpha=0.5$. 
to the supersonic flight conditions.

For a baseline engine, higher CPR limits the heat addition in the main burner due to the higher inlet temperature of the compressed air. The situation is even worse at the supersonic flight when the ram effect introduces at least a pressure rise of 2 times higher than the ambient pressure, which raises further the inlet temperature of the main burner. The consequence is the decreasing trend of thermal efficiency as shown in Figure 6.8c. The good news is that ITB remedies this problem by allowing a secondary heat addition at a pressure relatively higher than the pressure of an afterburner at some military engines. Also, ITB engines produce more ST at the same amount of fuel consumption as shown in Figure 6.8f. In fact, unlike in the subsonic flight, the ITB engine with $T_{t 4.5}$ of $1300 \mathrm{~K}$ not only possesses a much better thermal efficiency, but also yields an improved overall efficiency. In other words, ITB can be a potential improvement to the military supersonic turbofan engine performance. 

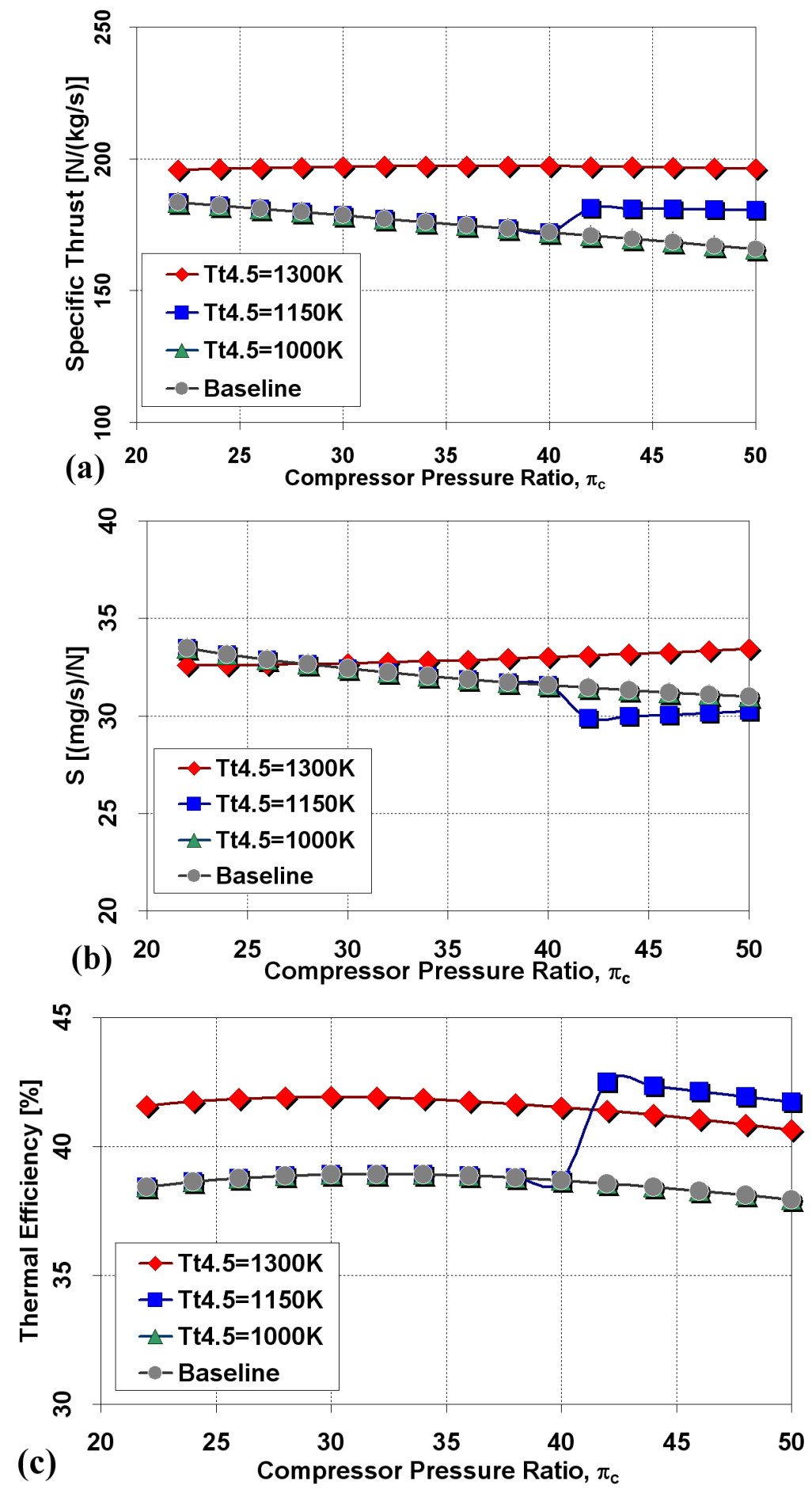

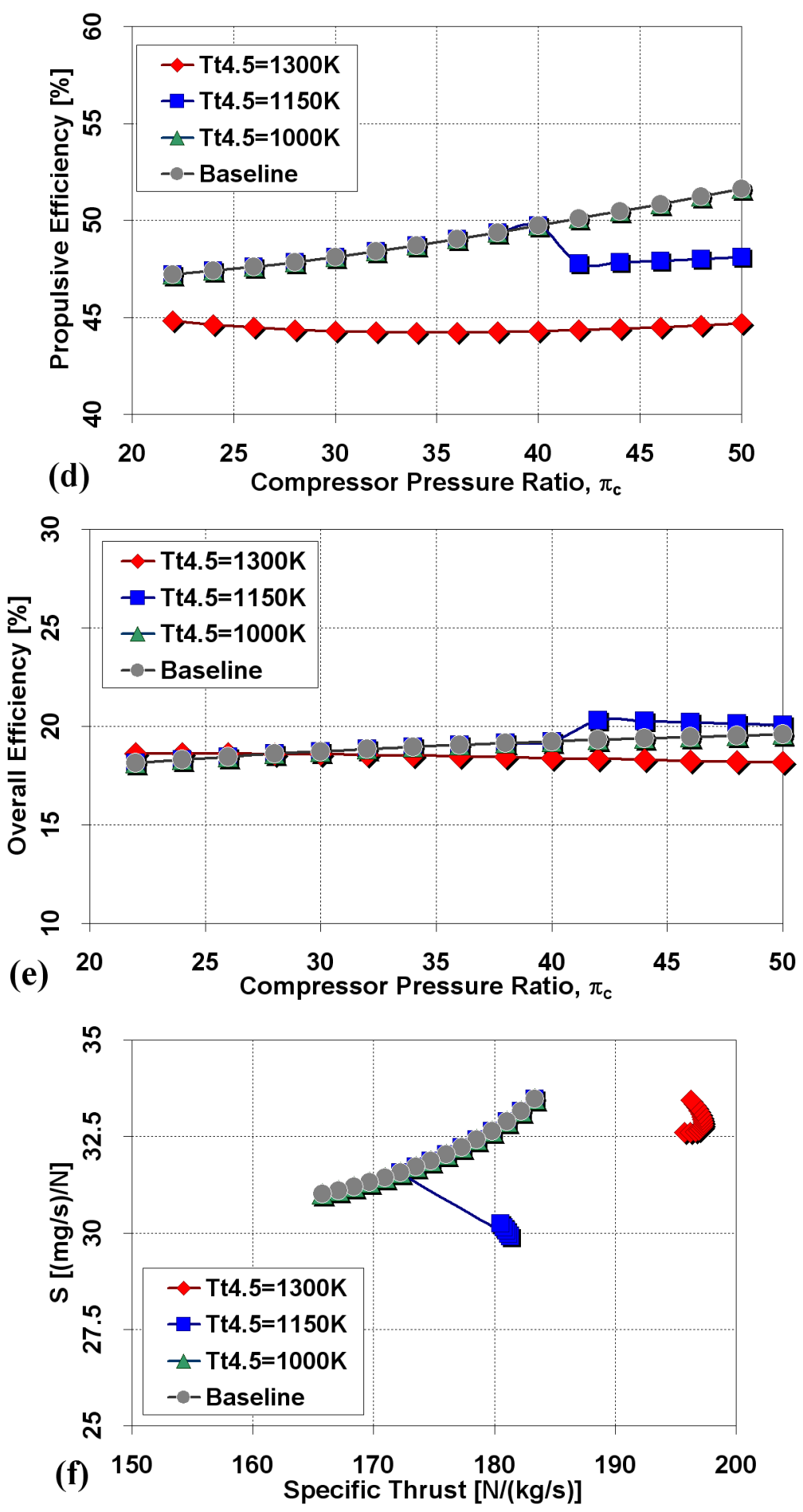

Figure 6.7: Performance comparison of turbofan engines versus compressor pressure ratio at an altitude of $10 \mathrm{~km}, M_{0}=0.85, \pi_{f}=1.3, \pi_{c L}=1.387, \alpha=0.5$, and $T_{t 4}=1600 \mathrm{~K}$. 

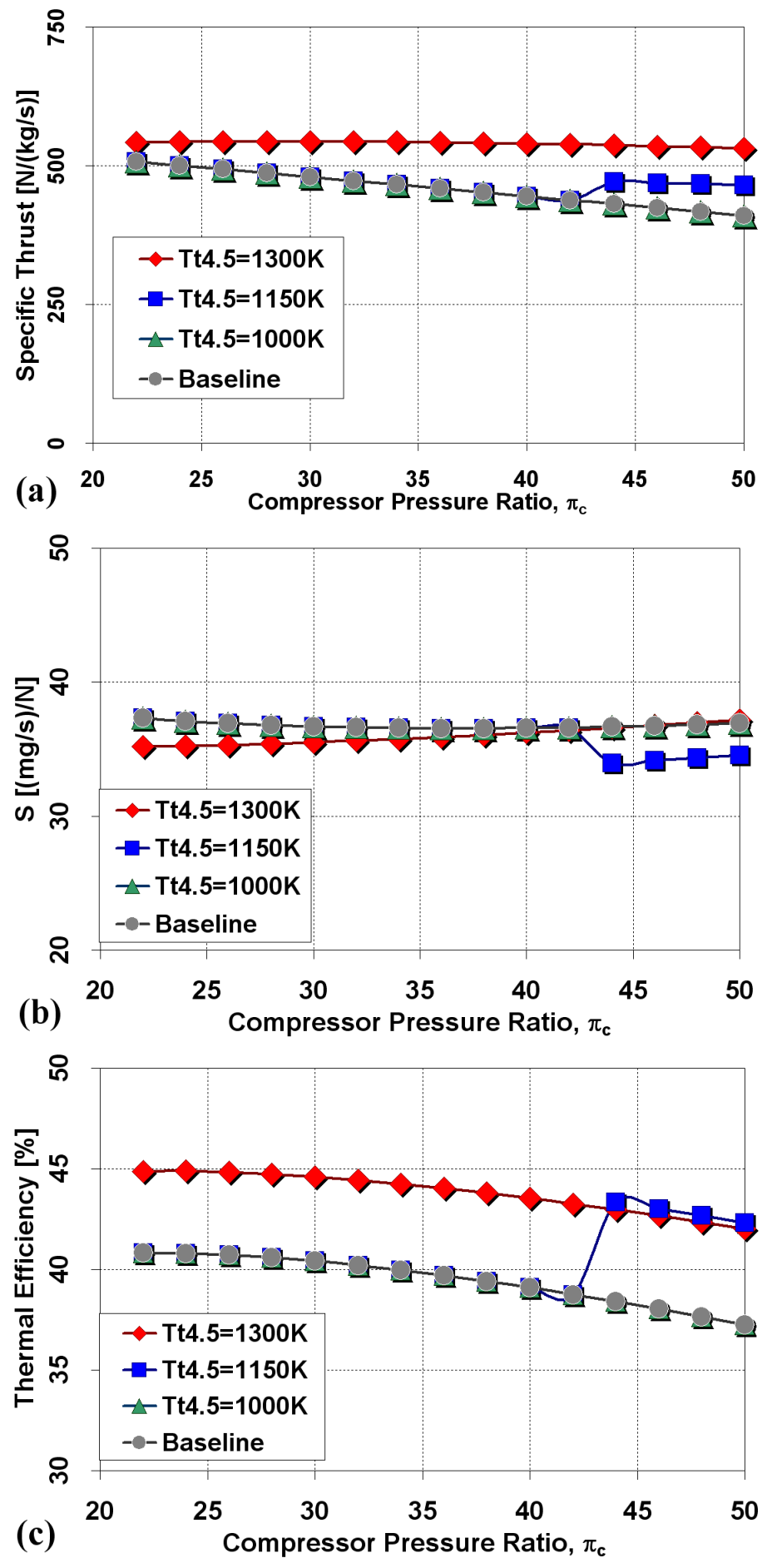

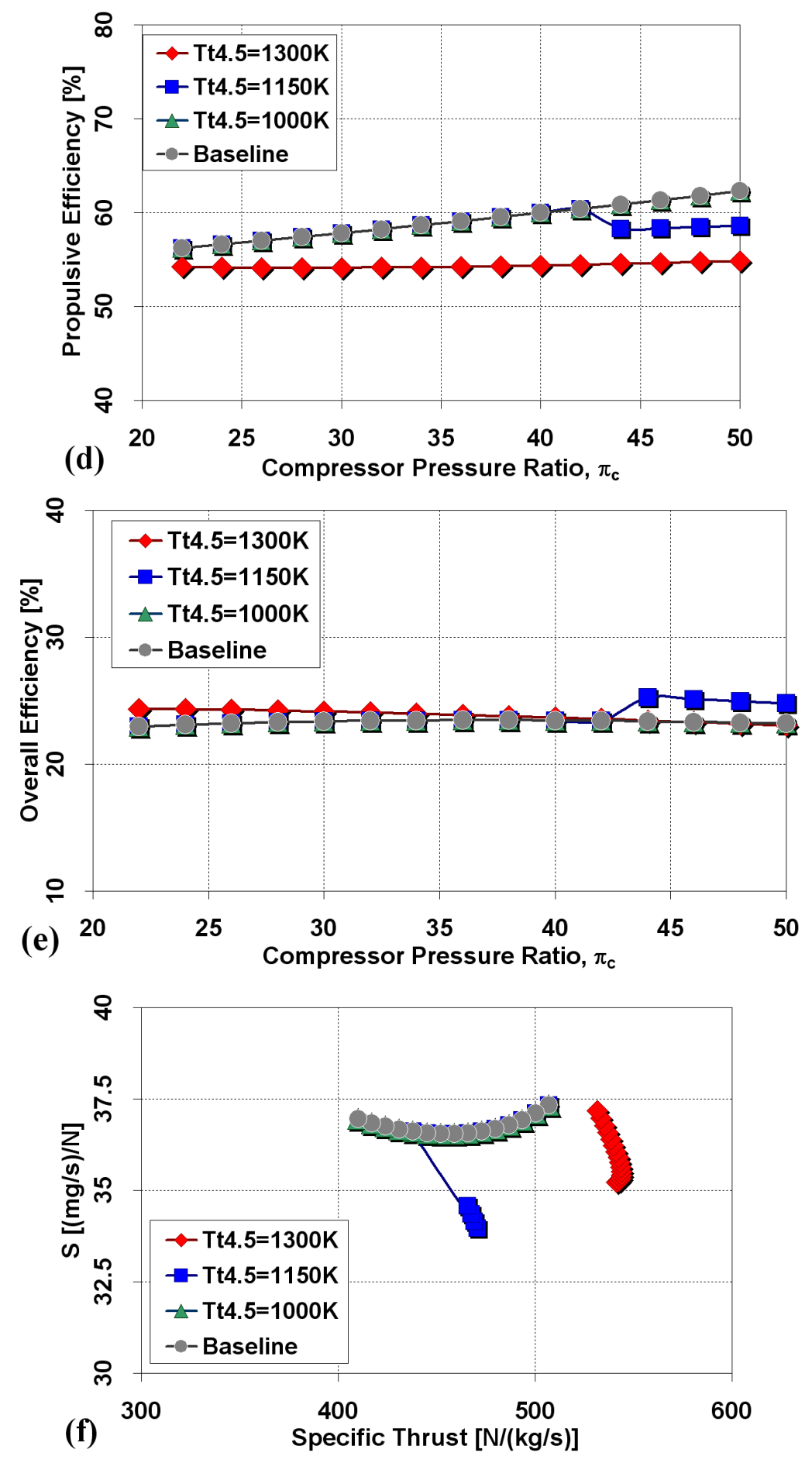

Figure 6.8: Performance comparison of turbofan engines versus compressor pressure ratio at an altitude of $10 \mathrm{~km}, M_{0}=1.2, \pi_{f}=3.0, \pi_{c L}=1.387, \alpha=0.5$, and $T_{t 4}=1600 \mathrm{~K}$. 


\subsubsection{Effect of Fan Pressure Ratio}

\section{Subsonic Flight}

Increasing FPR is a way to supply more energy to the bypass flow. In Saravanamuttoo et al. [45], military aircrafts may have two- or three-stages fan with FPR as high as 4.0 whereas civil aircrafts will always use a single-stage fan with FPR of about 1.5 to 1.8 .

Figures 6.9 a through 6.9 compare the engine performances of the baseline engine with three different ITB engines for varying FPR at a subsonic speed $\left(M_{0}=0.85\right)$ with $T_{t 4}$ of $1600 \mathrm{~K}$. The CPR and FBR are fixed at 28.48 and 4.0, respectively.

As shown in Figure 6.9 a, the value of ST of the baseline engine increases initially and starts decreasing at FPR equal to 2.5. It is because more work is extracted from LPT to fan in order to attain the higher FPR. The lower energy at the LPT exhaust stream also leads to a decrease in the average exit velocity of the engine core stream. Beyond that point, the decrease in engine core exit velocity is more than the increase in the fan exit velocity, thus lowering the total thrust level. This also explains the decreasing trend of the thermal efficiency.

Nevertheless, the secondary heat addition in ITB supplies enough energy to LPT in order to drive the larger fan. Furthermore, the overall efficiency of ITB engine (with $T_{t 4.5}$ of $1300 \mathrm{~K}$ ) at higher FPR is clearly seen when the value of $\mathrm{S}$ is lower than that of the baseline engine at FPR beyond 1.7. In addition, unlike the case with the increasing Mach number and CPR, Figures 6.9 and 6.9p also show us that there is an optimum value of FPR for the case being studied, which will maximize ST and minimize S. 


\section{Supersonic Flight}

For supersonic flight, the performance trend is qualitatively similar to those of the subsonic flight mentioned above, i.e., a better overall performance of the ITB engine over the baseline engine, as shown in Figure 6.10. 

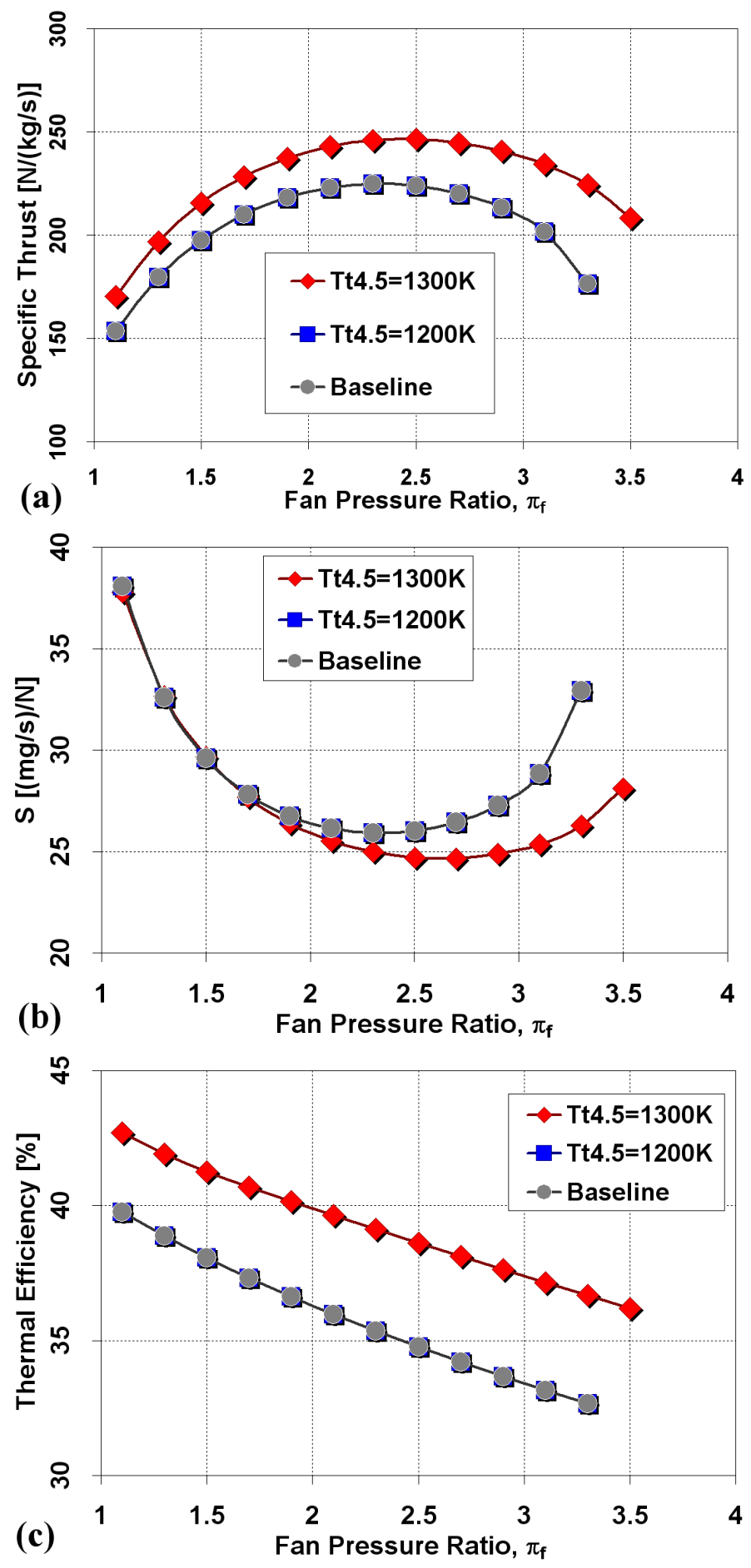

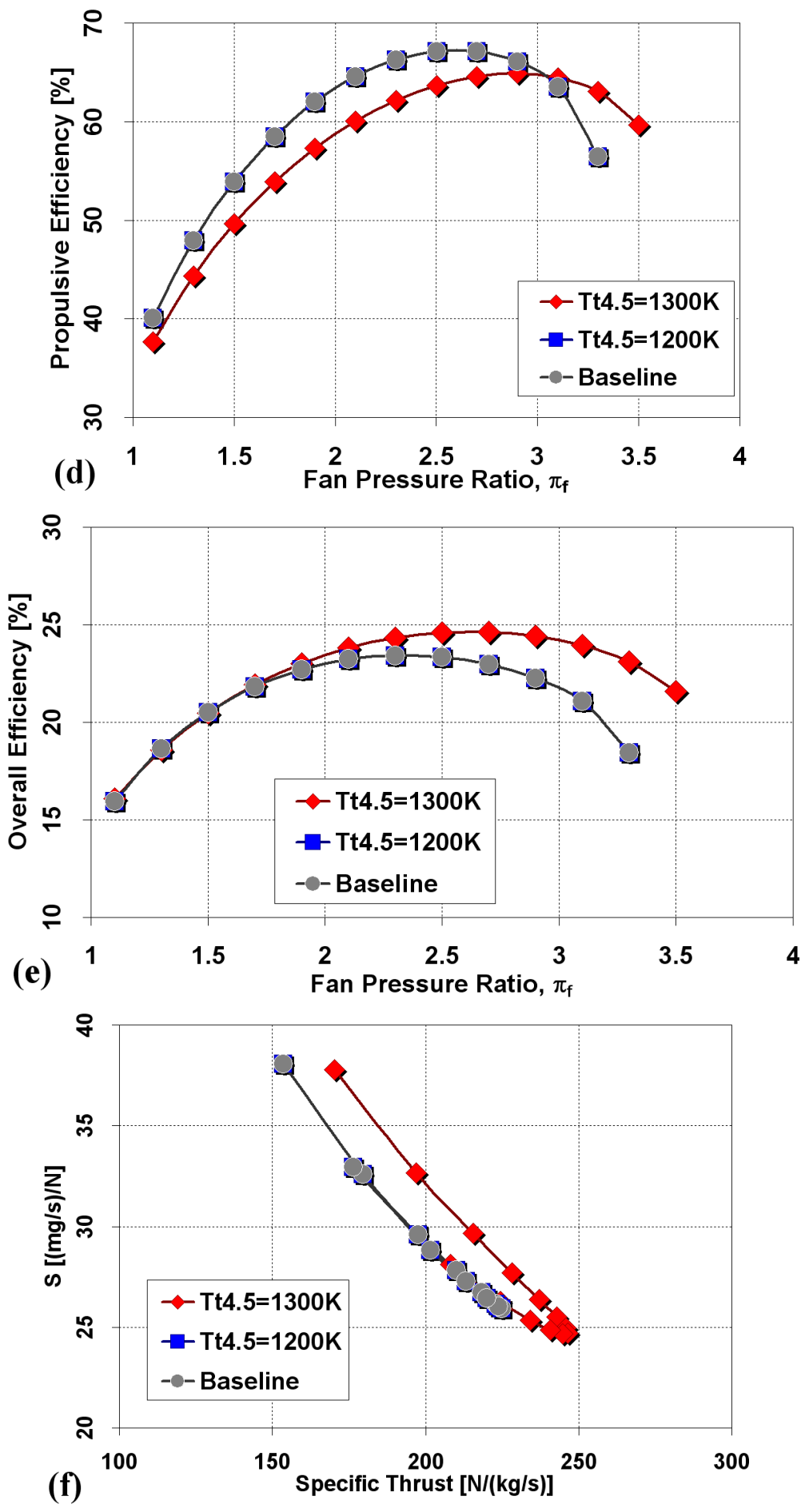

Figure 6.9: Performance comparison of turbofan engines versus fan pressure ratio at an altitude of $10 \mathrm{~km}, M_{0}=0.85, \pi_{c}=28.48, \pi_{c L}=1.387, \alpha=4.0$, and $T_{t 4}=$ $1600 \mathrm{~K}$. 

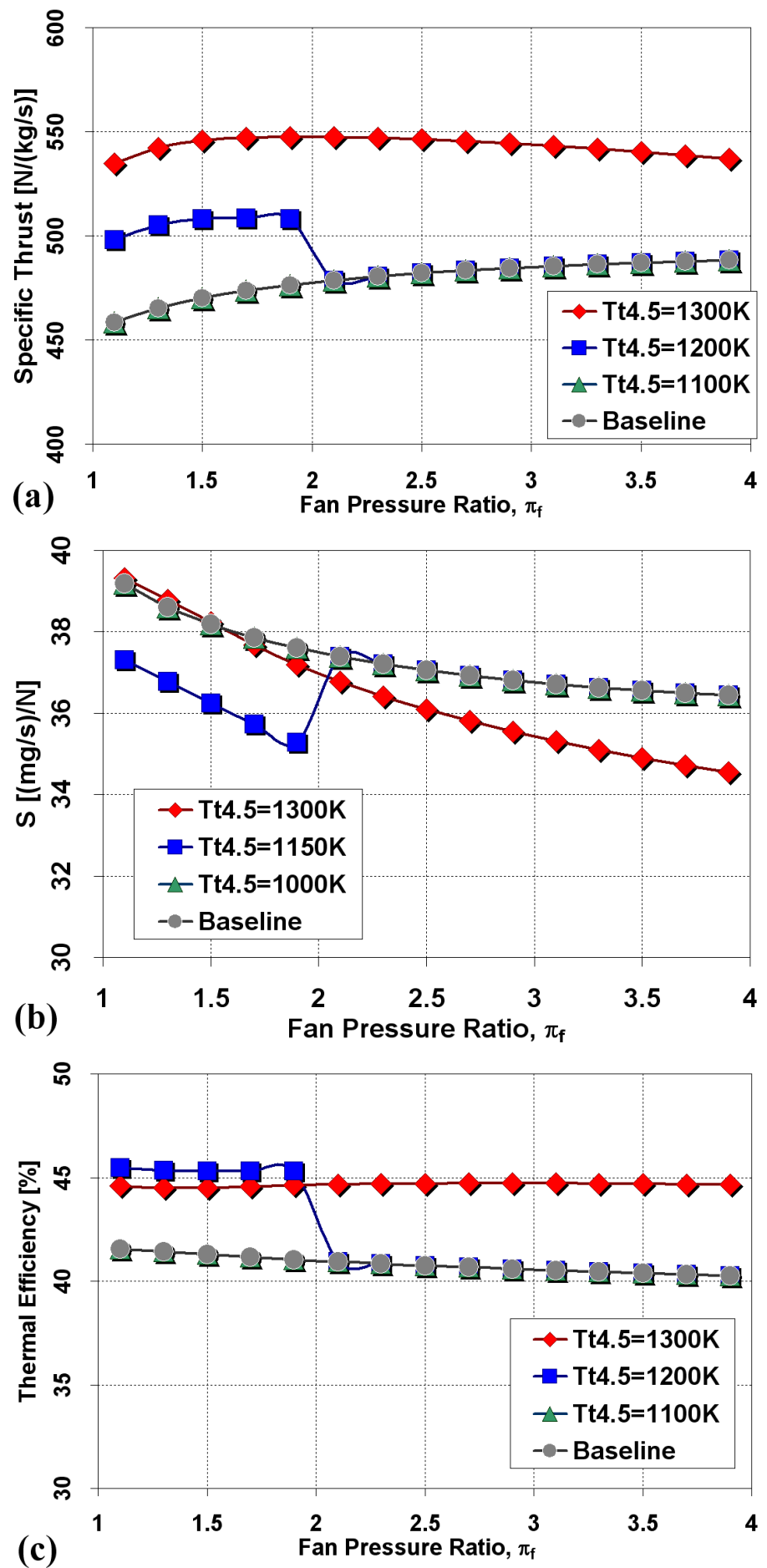

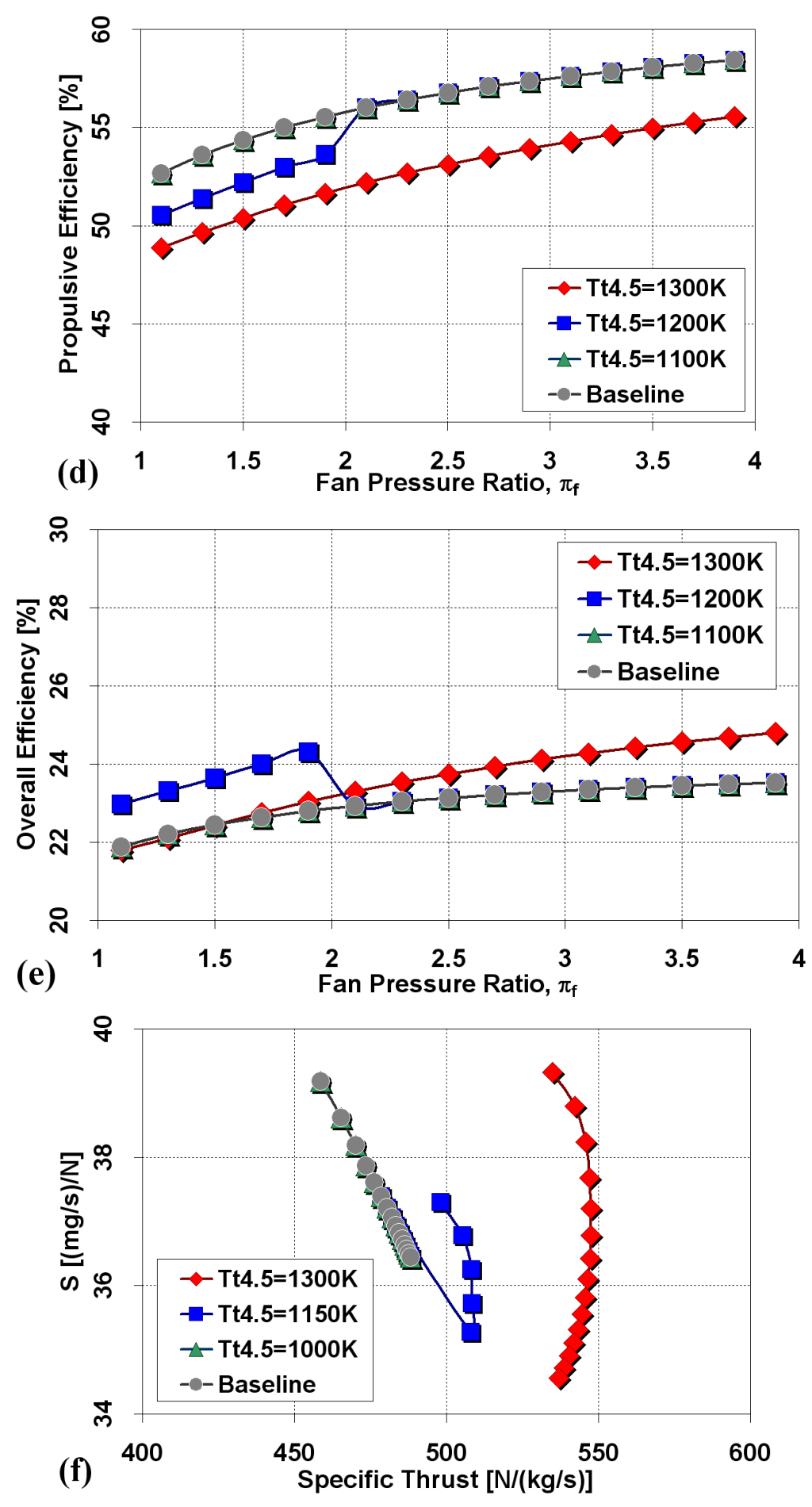

Figure 6.10: Performance comparison of turbofan engines versus fan pressure ratio at an altitude of $10 \mathrm{~km}, M_{0}=1.2, \pi_{c}=28.48, \pi_{c L}=1.387, \alpha=0.5$, and $T_{t 4}=1600 \mathrm{~K}$. 


\subsubsection{Effect of Fan Bypass Ratio}

\section{Subsonic Flight}

Figure 6.11a through 6.11 show the performance comparisons for varying FBR at $M_{0}$ of 0.85 . Clearly, as the FBR increases, the characteristics of the baseline engine are the decreasing trends of ST, S and thermal efficiency. The $\mathrm{ST}$ is decreasing because the total air flow rate is increasing as a result of the increasing BPR. Despite the decreasing trend, it is widely known that the higher FBR tends to improves the propulsive efficiency, which makes up for the reduction in thermal efficiency [49], and thus the better overall efficiency. With an addition of ITB, the engine characteristics remains the same with an improvement in ST and thermal efficiency.

\section{Supersonic Flight}

The performance comparisons for varying FBR at supersonic speed $\left(M_{0}\right.$ of 1.2) are shown in Figure 6.12 through 6.12 . The baseline engine ceases to produce thrust at FBR beyond 3.8 (moderate FBR). However, adding ITB to the engine will not only widen its operation range up to high FBR (close to 5.0), but also gain more than $12 \%$ increase in ST accompanied by a more than $4 \%$ decrease in $\mathrm{S}$ as $\mathrm{FBR}$ increases. It may be unfeasible to operate a supersonic engine at moderate or high FBR. Nevertheless, the advance in turbine

technology (increased $T_{t 4}$ ) will soon allow using a fan with FBR larger than those traditionally used in supersonic turbofan engines ( $\mathrm{FBR}=0.5$ or less) while maintaining a reasonably small frontal area [41]. 

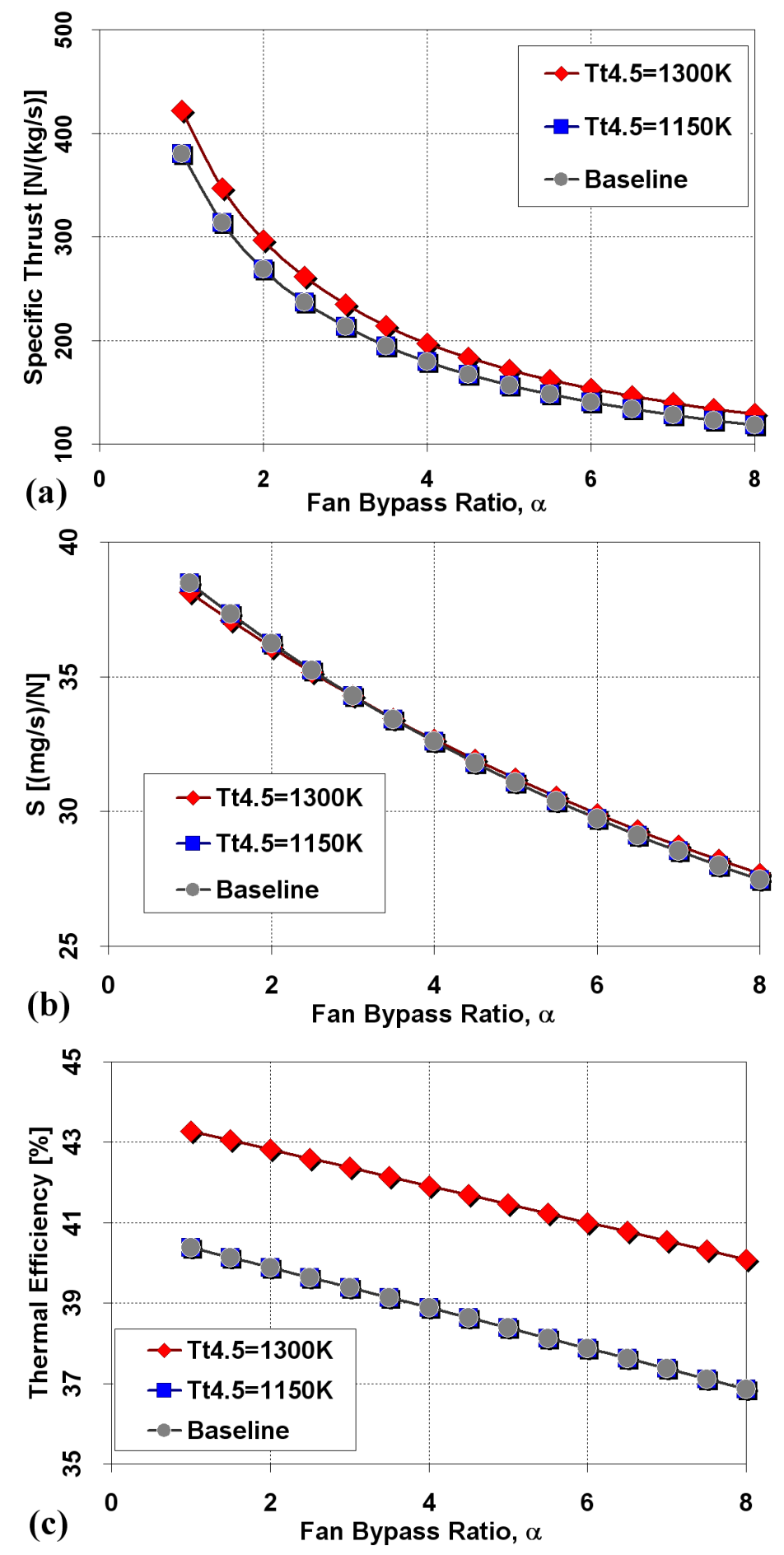

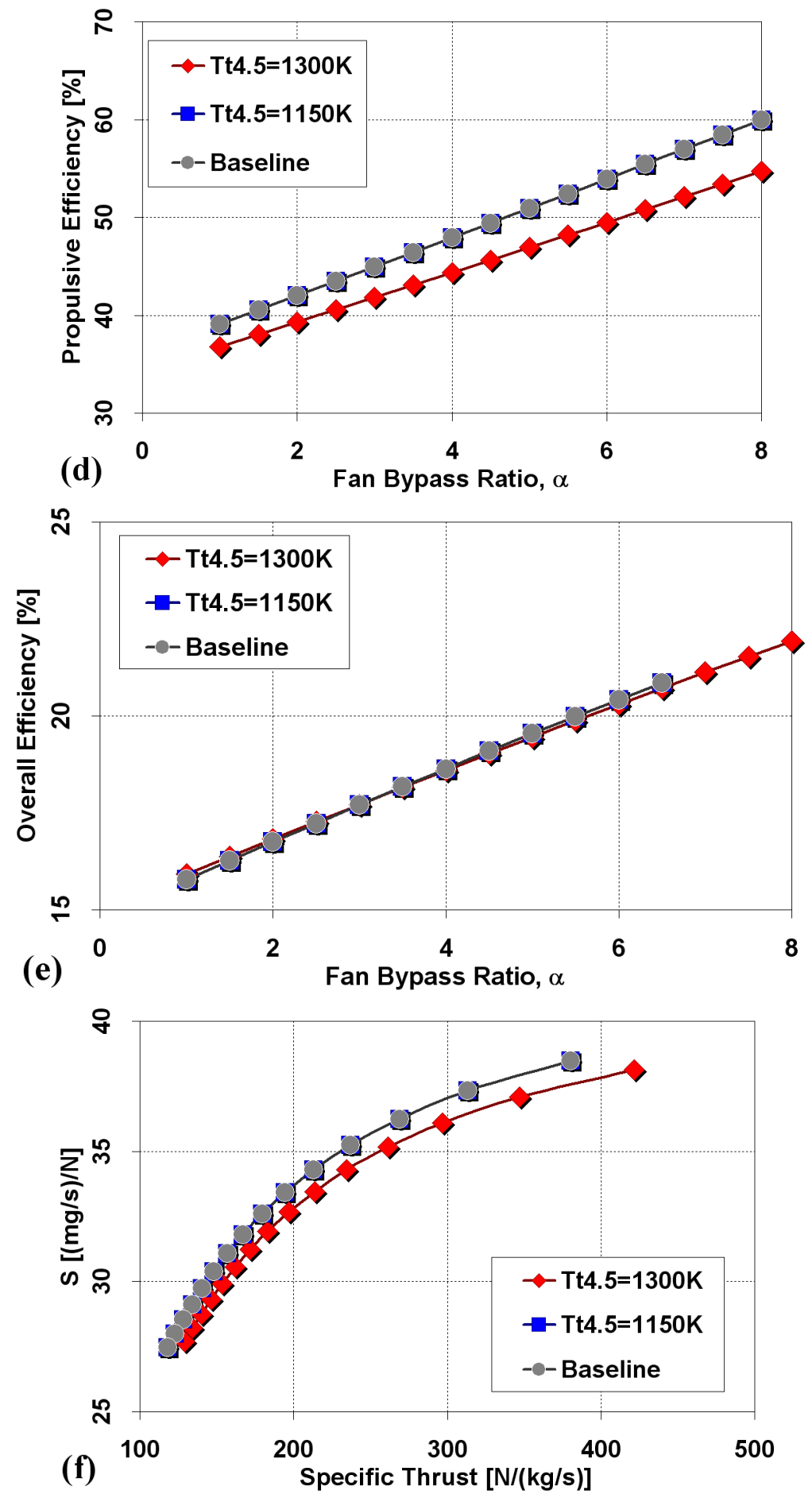

Figure 6.11: Performance comparison of turbofan engines versus fan bypass ratio at an altitude of $10 \mathrm{~km}, M_{0}=0.85, \pi_{c}=28.48, \pi_{c L}=1.387, \pi_{f}=1.3$, and $T_{t 4}=$ $1600 \mathrm{~K}$. 

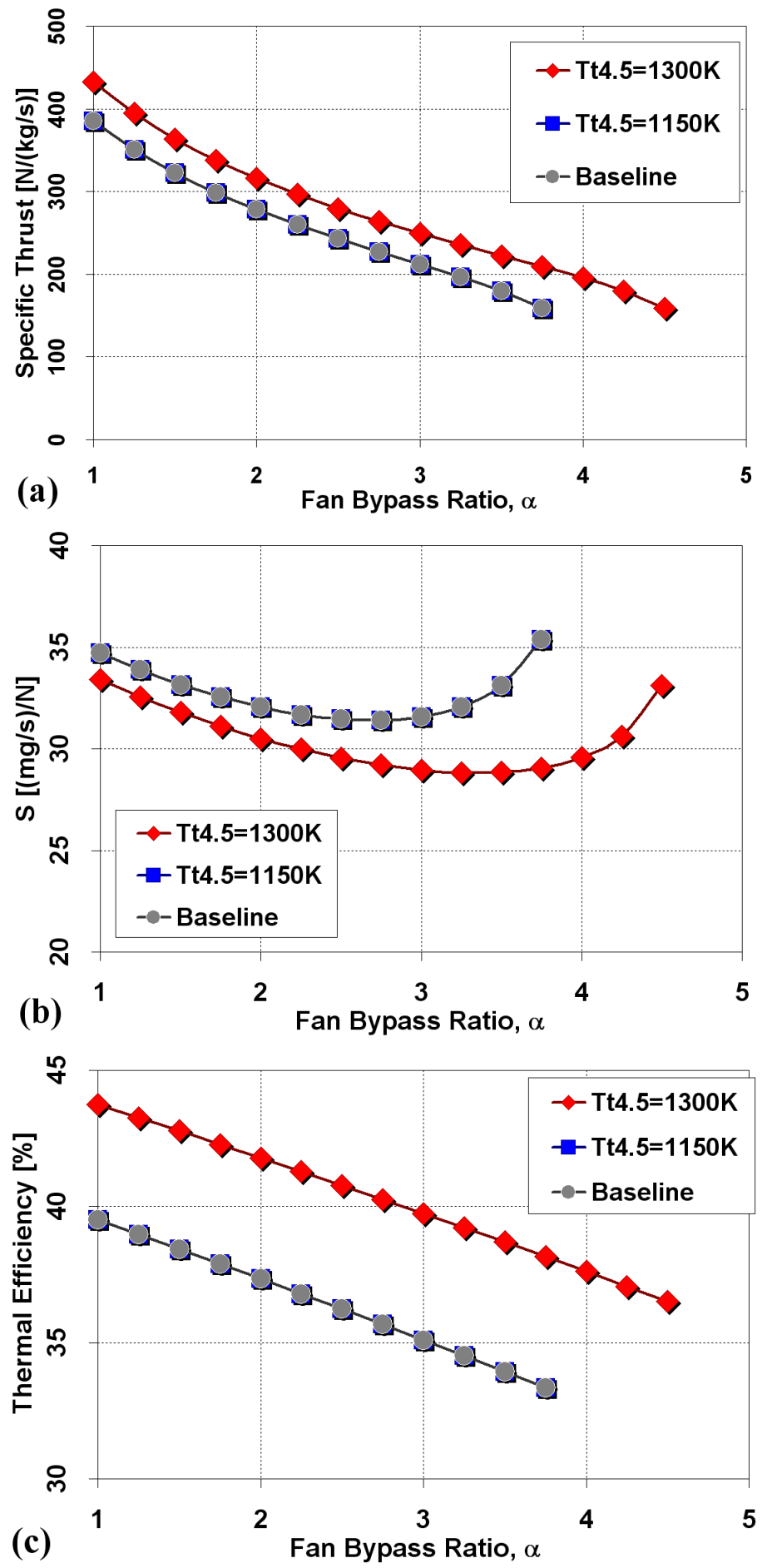

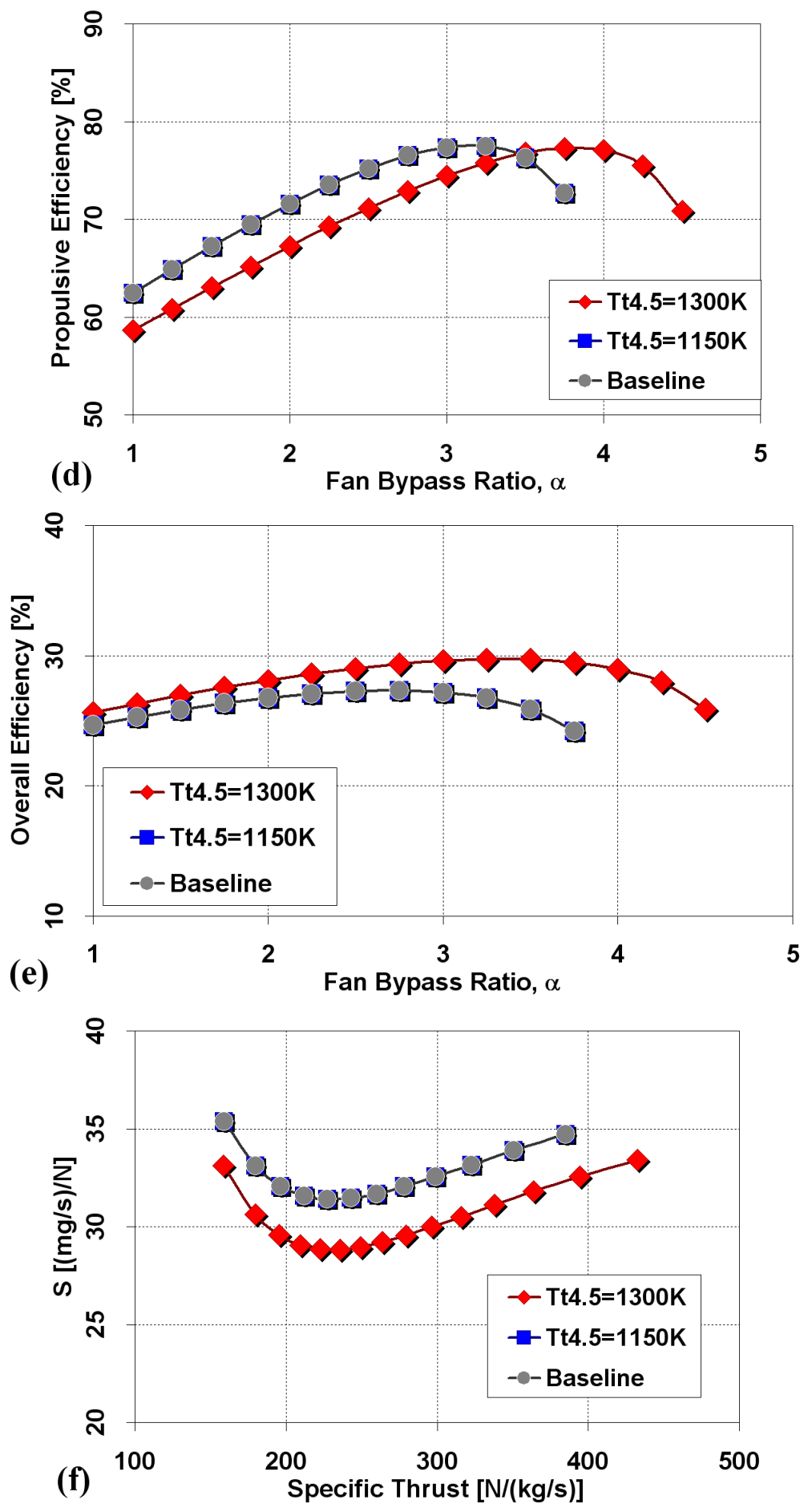

Figure 6.12: Performance comparison of turbofan engines versus fan bypass ratio at an altitude of $10 \mathrm{~km}, M_{0}=1.2, \pi_{c}=28.48, \pi_{c L}=1.387, \pi_{f}=3.0$, and $T_{t 4}=$ $1600 \mathrm{~K}$. 


\subsubsection{Advantages of Operation of Main Burner and ITB Together}

An important target in the engine design with ITB is the reduced amount of thermal $N O_{x}$. One way to achieve this is to lower the peak temperature inside the burner. We first consider three engines in the following discussion: engine $\mathrm{A}$ (i.e., a baseline engine with $T_{t 4}$ set to $1300 \mathrm{~K}$ ), engine $\mathrm{B}$ (i.e., a baseline engine with $T_{t 4}$ set to $1600 \mathrm{~K}$ ), and engine $\mathrm{C}$ (i.e., basically engine $\mathrm{A}$ with an addition of ITB, where $T_{t 4.5}$ is set to $1300 \mathrm{~K}$ ). The engine of target among these three is the engine $\mathrm{C}$, which is compared to its baseline engine (i.e., engine $\mathrm{A}$ ) and another baseline engine with a higher peak engine temperature (i.e., engine $\mathrm{B}$ ). Figures 6.13 through 6.13. demonstrate the performance comparisons of these three engines for flight Mach number of 0 to 2.

In the previous discussions, it becomes clear that as the fluid undergoes a secondary combustion, a higher ST results with an improvement in thermal efficiency. Therefore, it is expected to see engine $\mathrm{C}$ having better ST and improved thermal efficiency than its baseline engine (engine A). On the other hand, despite having a lower efficiency than engine $\mathrm{B}$ due to a lower peak temperature (i.e., 1300K) inside the burners, engine $\mathrm{C}$ has advantages over engine B. Its advantages include higher ST and no turbine cooling required in HPT. Small amounts of cooling air may be required if a low-technology blade material is used, or if higher values of $T_{t 4}$ and $T_{t 4.5}$ are specified.

In addition to the advantages mentioned above, engine $\mathrm{C}$ is also said to produce a lesser amount of thermal $N O_{x}$ due to the lower peak temperatures inside the burners. To verify this, a simple $N O_{x}$ model is derived and implemented into the existing code. All details of the $N O_{x}$ modeling can be found in Appendix $\mathrm{C}$. The comparison of $N O_{x}$ emission from each engine is depicted in Figure 6.14. For comparison, each EINOx is normalized by dividing EINOx by its corresponding 
$\mathrm{ST}$. One can clearly see that engine $\mathrm{C}$ produce very slightly $N O_{x}$ more than its baseline engine (engine A) due to the additional burning inside the ITB. Nevertheless, engine $\mathrm{C}$ produce much lesser $N O_{x}$ than engine $\mathrm{B}$ while producing higher ST. This finding addresses the feasibility of reducing the peak engine temperature (i.e., from $1600 \mathrm{~K}$ to $1300 \mathrm{~K}$ ) without sacrificing the power output, while definitely reducing the $N O_{x}$ emission. 

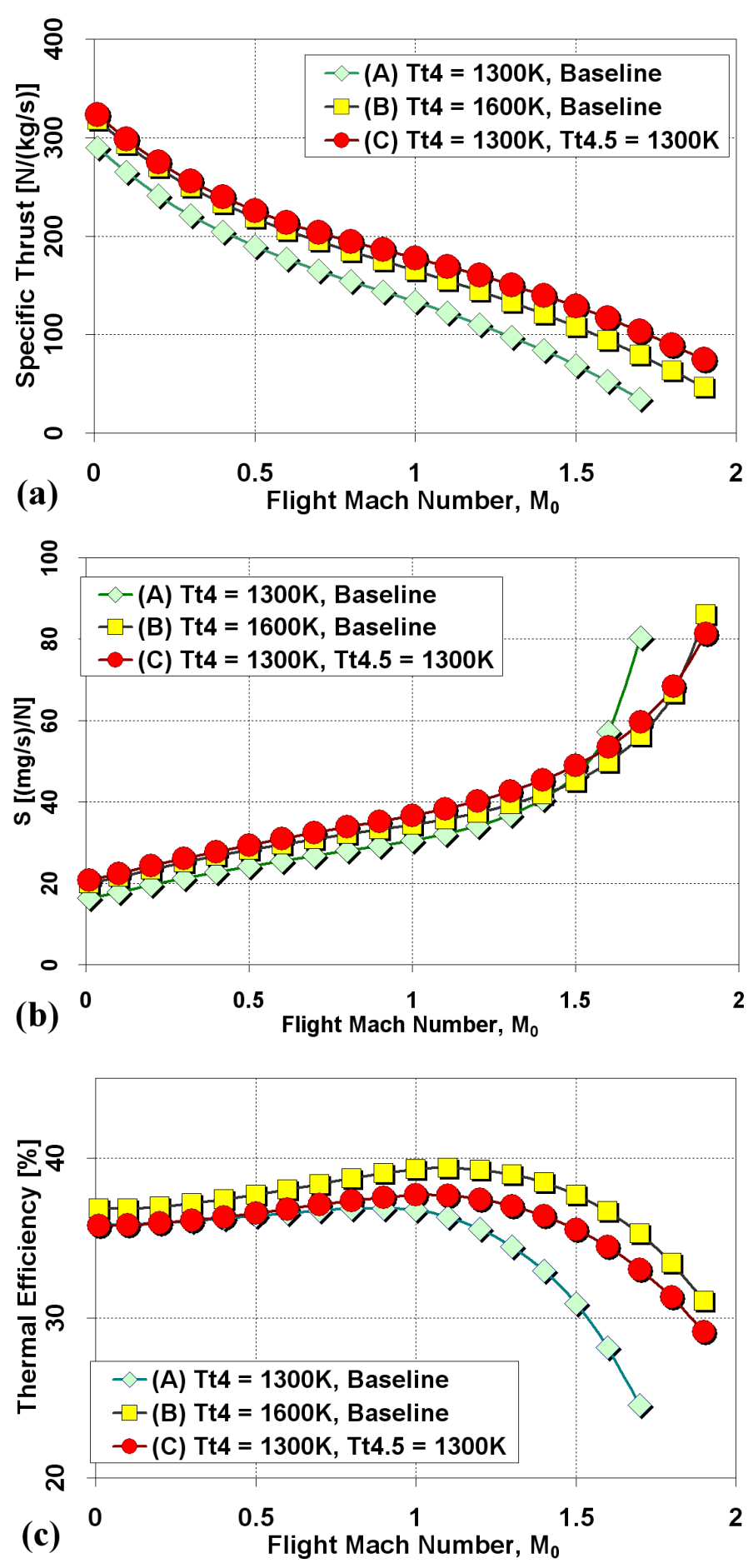

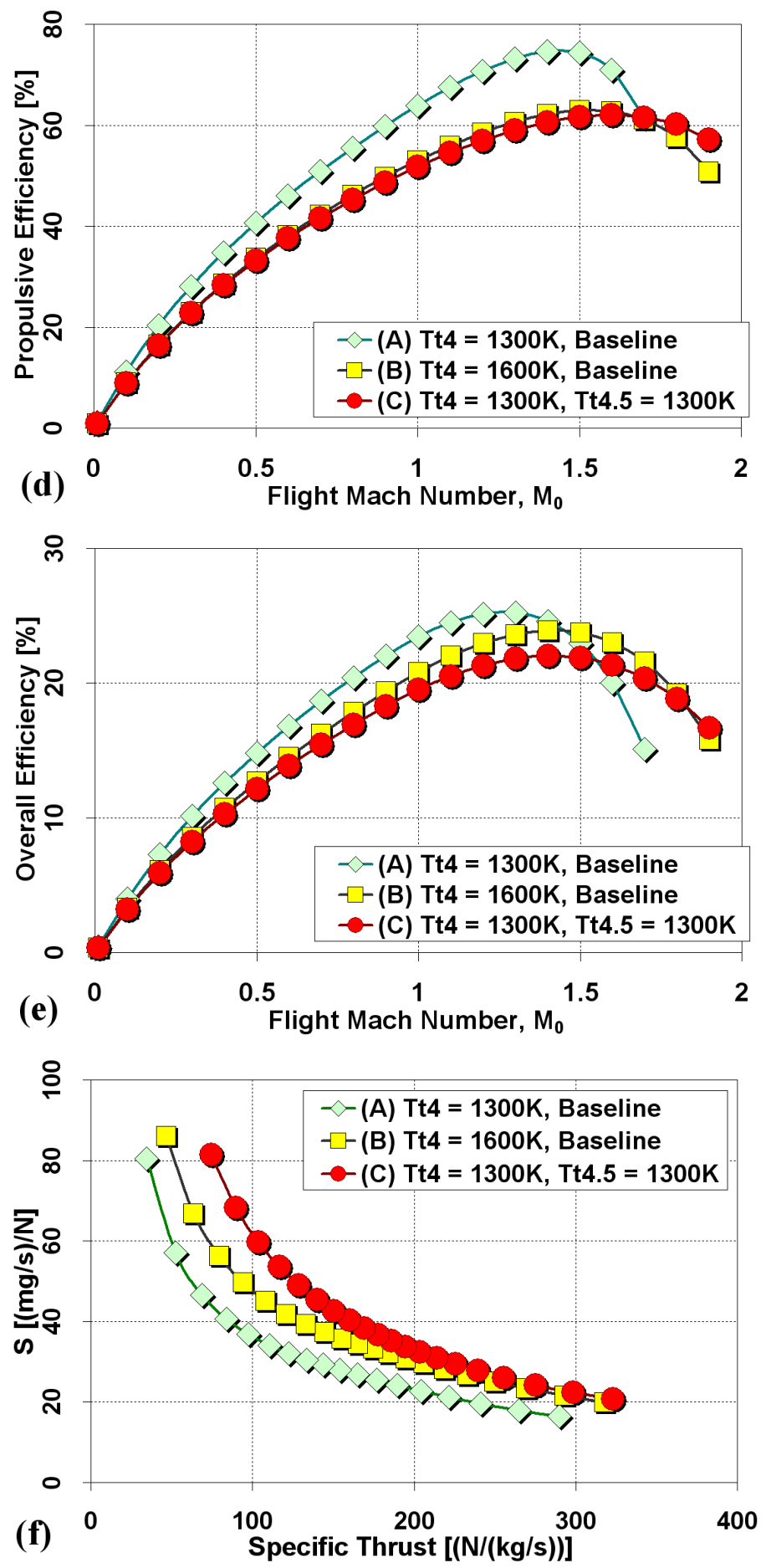

Figure 6.13: Performances of three turbofan engines versus $M_{0}, \pi_{f}=1.3, \pi_{c}=$ 28.48 , and $\alpha=4.0$. 


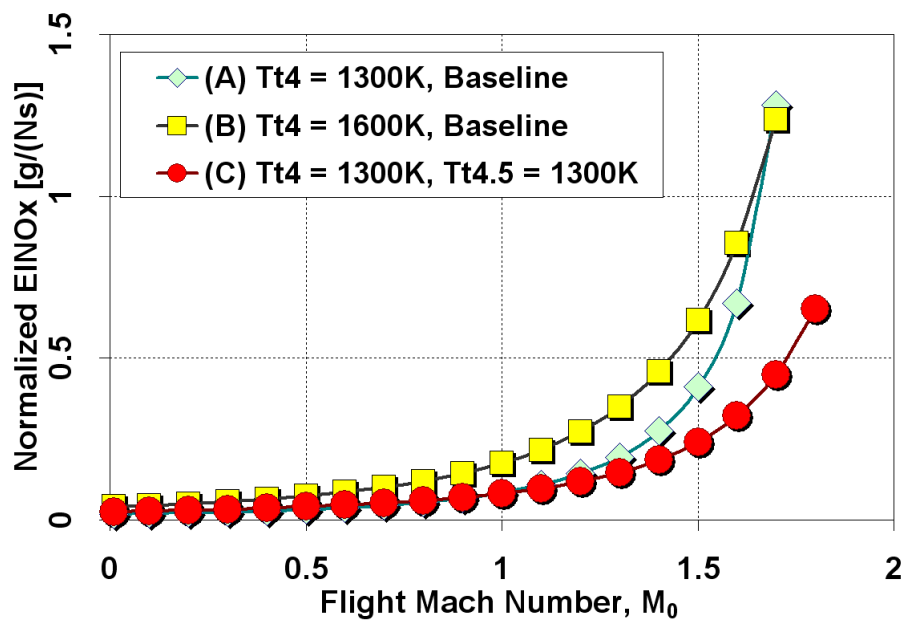

Figure 6.14: NOx emission of three turbofan engines versus $M_{0}, \pi_{f}=1.3, \pi_{c}=$ 28.48 , and $\alpha=4.0$.

\subsection{Results from Off-design Cycle Analysis}

\section{Engine Configuration}

Two sets of reference-point engine data at sea level static (SLS) condition are selected, i.e., case $\mathrm{A}$ and $\mathrm{B}$, as provided in Table 6.1. For each case, a conventional engine is considered as a baseline engine while a similar engine operating with an addition of ITB is termed as ITB engine. In addition, the component performance parameters, listed in Table J.1, are kept the same for both cases.

For full throttle operation, the maximum inlet HPT total temperature $\left(T_{t 4}\right.$ or main burner exit total temperature) and the LPT inlet total temperature ( $T_{t 4.5}$ or ITB exit total temperature) are set to the values as listed in Table 6.1. For partial throttle operation, the minimum thrust is set to 20 percent of the maximum thrust. 
Table 6.1: Design-point engine reference data

\begin{tabular}{lcc}
\hline \hline Description & \multicolumn{2}{c}{ Input value } \\
\hline \hline Reference Conditions & Case A & Case B \\
\hline Flight Mach number $\left(M_{0 R}\right)$ & 1.2 & 0.9 \\
Altitude $\left(h_{R}\right)$ & SLS & SLS \\
Main burner exit total temperature $\left(T_{t 4 R}, \mathbf{K}\right)$ & 1500 & 1500 \\
ITB exit temperature $\left(T_{t 4.5 R}, \mathbf{K}\right)$ & 1300 & 1300 \\
Compressor pressure ratio $\left(\pi_{c R}\right)$ & 25 & 28.48 \\
Fan pressure ratio $\left(\pi_{f R}\right)$ & 2.43 & 2.0 \\
Fan bypass ratio $\left(\alpha_{R}\right)$ & 0.73 & 4.0 \\
Mass flow rate $\left(\dot{m}_{0 R}, \mathbf{k g} / \mathbf{s}\right)$ & 118 & 550 \\
\hline \hline
\end{tabular}

\subsubsection{Full Throttle Performance}

\section{Low FBR}

Figures 6.15 a through 6.15 present the uninstalled performance of the turbofan engines with a low FBR of 0.73 operating at full throttle settings for case A. These figures show the variations of thrust $(F)$, S, and efficiencies with flight Mach number $\left(M_{0}\right)$. The solid lines represent ITB engine performance while the dashed lines represent baseline engine performance.

In Figure 6.15a, the ITB engine exhibits an increase in thrust over the baseline engine as $M_{0}$ increases. Because of more fuel injected into ITB in addition to the main burner, ITB engines do have slightly higher fuel consumption than the baseline engine. Nevertheless, adding ITB is still beneficial because the improvement in thermal efficiency (Fig. 6.15c) reflects that the gain in thrust offsets the slight increase in $\mathrm{S}$.

In Figures. 6.15 and 6.15; , both thrust and thermal efficiency curves exhibits a slope change at $M_{0}$ of about 1.2. The engine control system takes place at that operating point in order to limit the main burner exit temperature from exceeding the maximum inlet turbine temperature limit. 


\section{High FBR}

Figures 6.16a through 6.16 present the uninstalled performance of the turbofan engines with a high FBR of 4.0 operating at full throttle settings for case B. It is found that both engines have similar performance trends over the flight spectrum as in case A, except that the ITB engine has almost the same level of specific fuel consumption compared with the baseline engine. Another thing to notice is that the overall efficiencies of both engines start to deteriorate at very high Mach number. 

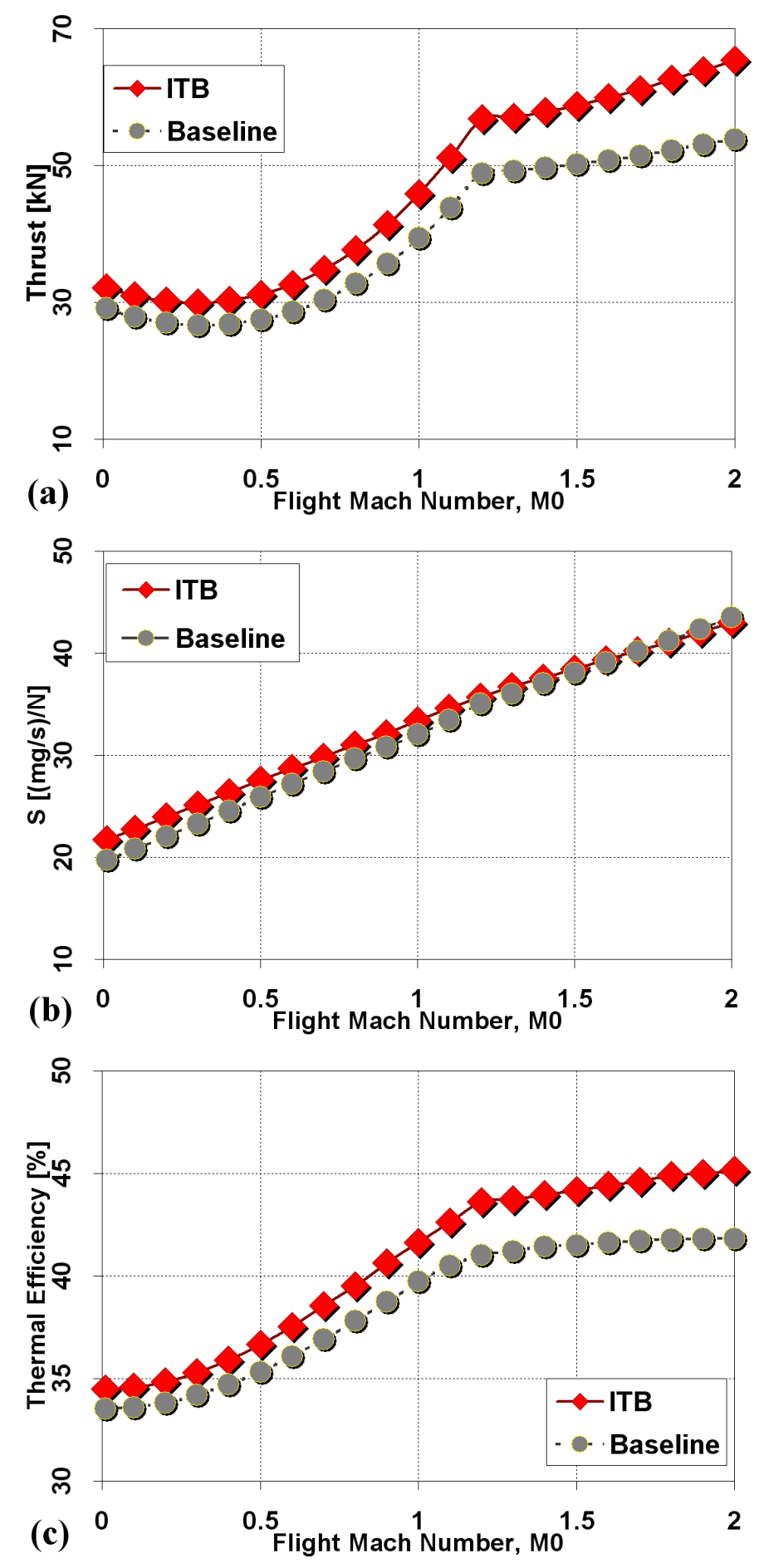

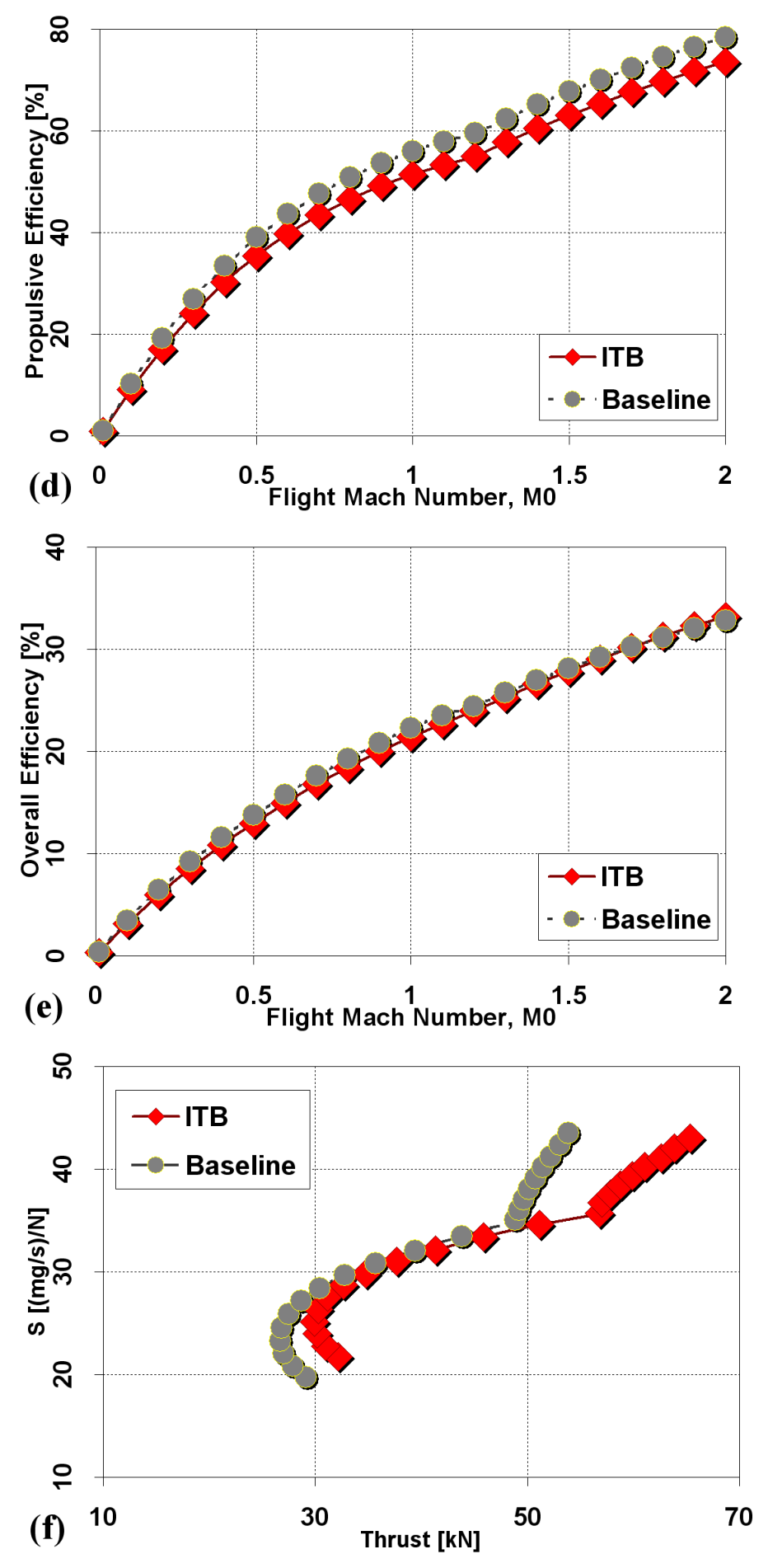

Figure 6.15: Full-throttle performance comparison of turbofan engines (case A) versus $M_{0}$ at an altitude of $10 \mathrm{~km}, \pi_{f R}=2.43, \pi_{c R}=25, T_{t 4 R}=1500 \mathrm{~K}, T_{t 4.5 R}=$ $1300 \mathrm{~K}, \dot{m}_{0 R}=118 \mathrm{~kg} / \mathrm{s}$, and $\alpha=0.73$. 

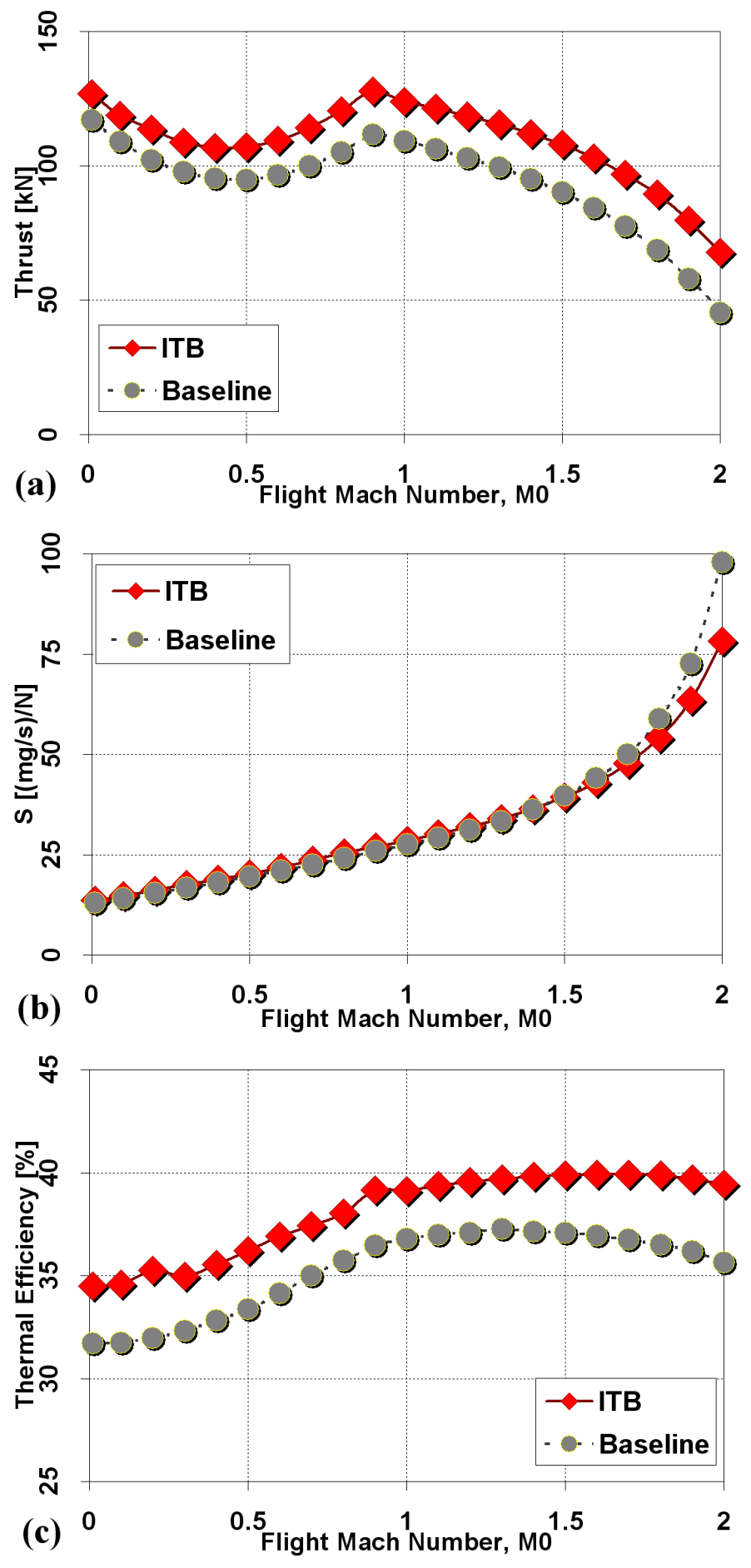

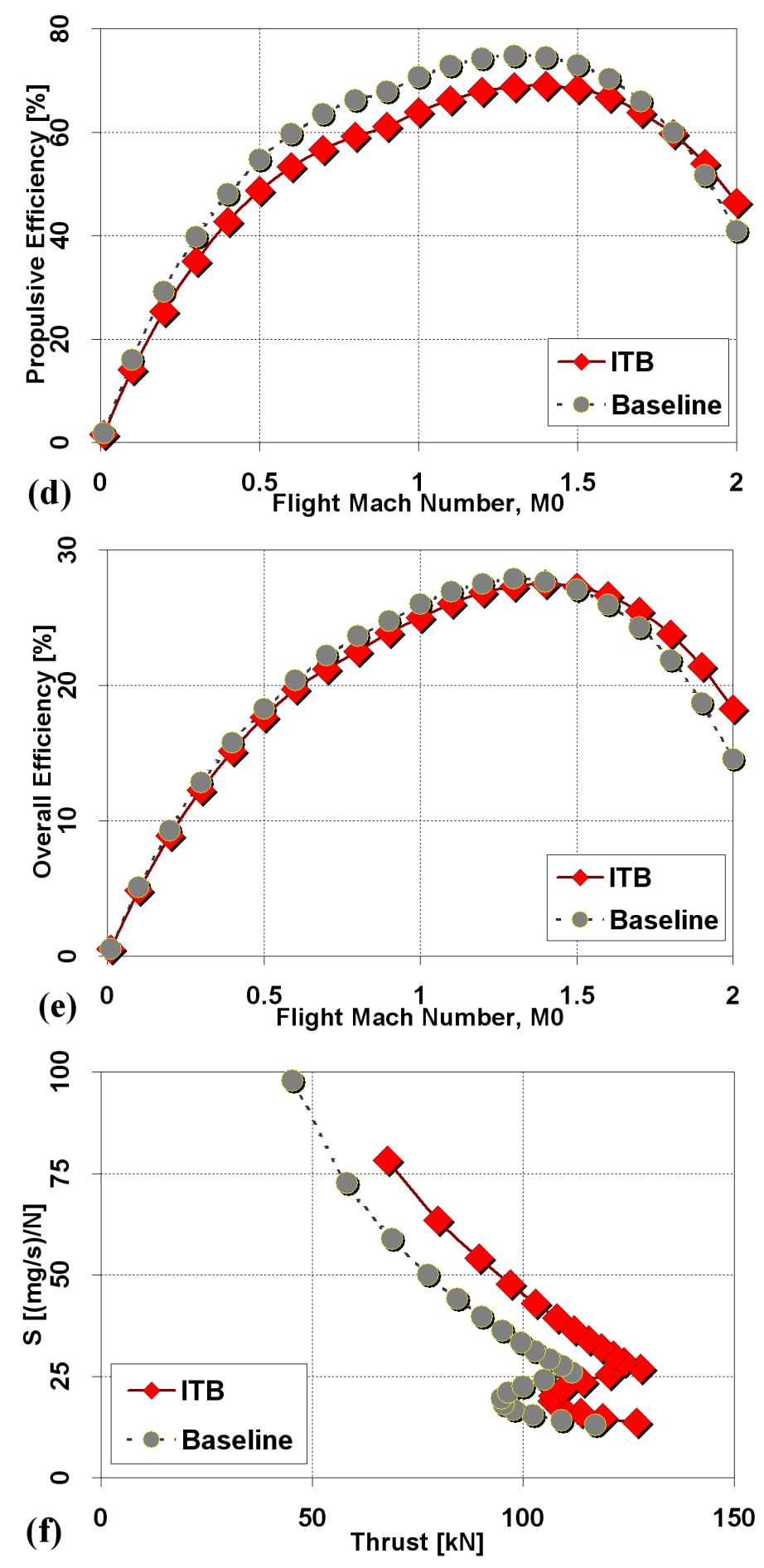

Figure 6.16: Full-throttle performance comparison of turbofan engines (case B) versus $M_{0}$ at an altitude of $10 \mathrm{~km}, \pi_{f R}=2, \pi_{c R}=28.48, T_{t 4 R}=1500 \mathrm{~K}, T_{t 4.5 R}=$ $1300 \mathrm{~K}, \dot{m}_{0 R}=550 \mathrm{~kg} / \mathrm{s}$, and $\alpha=4.0$. 


\subsubsection{Partial Throttle Performance}

Figures 6.17 (case $\mathrm{A}$ ) and 6.18 (case B) show the 'S versus $\mathrm{F}$ ' and ' $\eta_{t h}$ versus $F^{\prime}$ curves at partial throttle settings for two different values of $M_{0}$ at an altitude of $10 \mathrm{~km}$. Case $\mathrm{A}$ represents the engines with a low FBR whereas case B represents the engines with a high FBR. As seen clearly in Figs. 6.17a and 6.18 a, the partial throttle performance curves for ITB engines preserve the classical hook shape that is known as throttle hook in the propulsion community. As the throttle is reduced (i.e., the thrust is decreased) until the ITB is turned off (which appears as a discontinuity in each curve), it results in a change in slope from a linear curve to a spline. This change is accompanied by an abrupt increase in $\mathrm{S}$ and a drop in thrust.

According to Figs. 6.17a and 6.18a, it is clearly noticed that adding ITB further extends the engine operational range by producing higher thrust levels than that of the baseline engine. Within these higher thrust curves, the fuel consumption increases linearly with increasing thrust until it reaches a local maximum point, which represents the full throttle operation. Depending on the engine configuration and flight conditions, this maximum point may or may not have a $\mathrm{S}$ value higher than the $\mathrm{S}$ value of a baseline engine at its full throttle operation.

For example, the local maximum points for the case-A ITB engine with $M_{0}$ of 0.9 and 1.2 (Fig $\left.6.17 \mathrm{a}\right)$ have always higher $\mathrm{S}$ levels than that of baseline engine. This is shown in Figure 6.15a, where the case-A ITB engine's full throttle operations at both levels of $M_{0}$ yield a very high fuel consumption.

Nevertheless, Figure $6.18 \mathrm{a}$ shows that the case-B ITB engine operating at full throttle condition exhibits relatively lower $\mathrm{S}$ values at two different $M_{0}$ 's compared to case A. Therefore, for some applications, for instance, the engine with a low FBR like in case A, it might be better to operate the ITB engine at 
partial throttle settings (i.e., lower $\left.T_{t 4.5}\right)$ to avoid burning extra fuel while still achieving modest thrust augmentation. This will certainly provide fuel saving to many aircraft engines, which normally run at partial throttle settings during cruise operations at high altitude.

As shown in Figures. 6.17b-c and 6.18b-c, the thermal efficiency of ITB engine is greatly improved over the baseline engine when ITB is on. However, its variation within the extended operational range is relatively small. This finding confirms the advantage of running the engine at partial load condition rather than at full load condition.

\subsubsection{Mission Analysis}

A systematic mission study of the fuel consumption is performed to reveal the advantage of saving fuel by adding ITB. However, at this preliminary design phase, the engine manufacturer's published data is often unavailable; therefore, the off-design engine model like this one can be used to give a preliminary estimate of fuel consumption in each mission phase [34]. A 5\% installation loss is accounted to give the mission analysis fuel consumption.

For the following mission study, only case A is considered. For simplicity, only critical mission phases and segments are selected. Each selected mission leg is judged to be critical because it has a high fuel consumption and is an extreme operating condition [34]. In each mission leg, the ITB engine is operating at partial throttle settings to avoid burning extra fuel as previously discussed.

Table 6.2 contains a summary of the mission performance of ITB engine (case A) as compared to baseline engine in term of fuel consumption. Each aircraft has an initial take-off weight of 24,000 lbf. It is found that ITB engine uses less fuel in all phases. Particularly, the fuel consumption in the Warm-up (1-2) phase is significantly less. This calculation also shows that ITB engine 

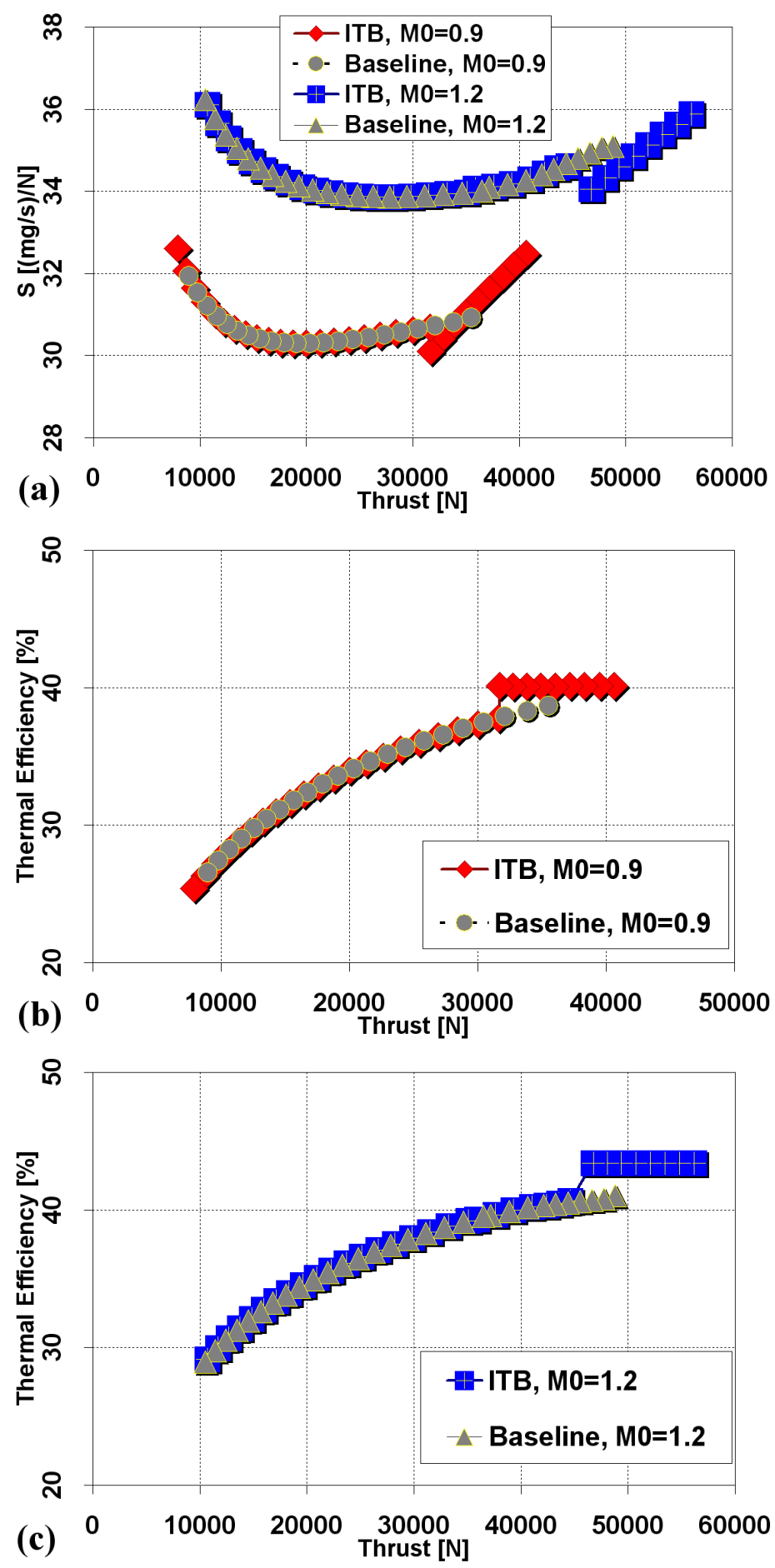

Figure 6.17: Partial-throttle performance of turbofan engine (case $\mathbf{A}$ ) at an altitude of $10 \mathrm{~km}, \pi_{f R}=2.43, \pi_{c R}=25, T_{t 4 R}=1500 \mathrm{~K}, T_{t 4.5 R}=1300 \mathrm{~K}, \dot{m}_{0 R}=118$ $\mathrm{kg} / \mathrm{s}$, and $\alpha=0.73$. 

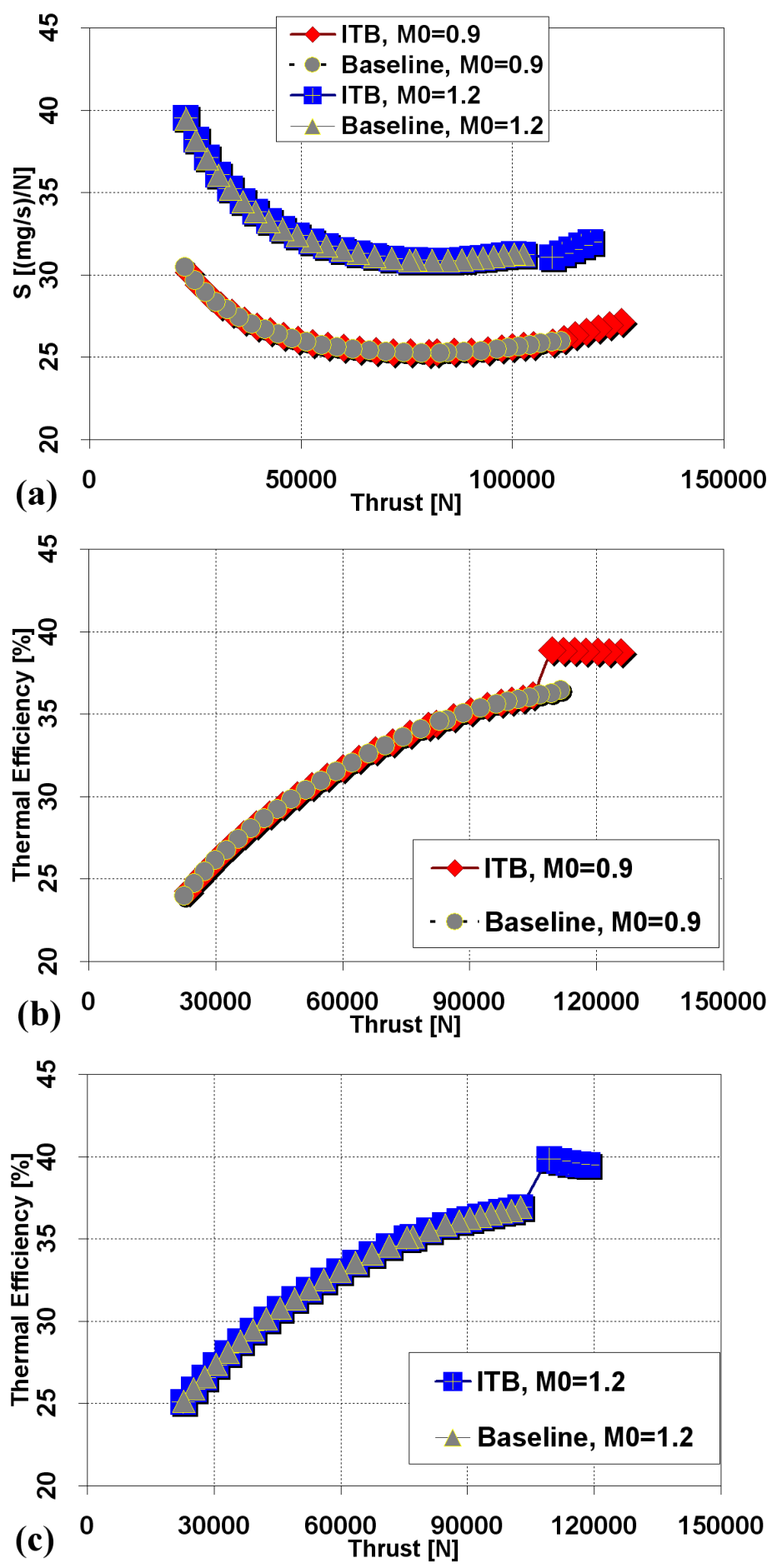

Figure 6.18: Partial-throttle performance of turbofan engine (case $\mathbf{B}$ ) at altitude of $10 \mathrm{~km}, \pi_{f R}=2, \pi_{c R}=28.48, T_{t 4 R}=1500 \mathrm{~K}, T_{t 4.5 R}=1300 \mathrm{~K}, \dot{m}_{0 R}=550 \mathrm{~kg} / \mathrm{s}$, and $\alpha=4.0$. 
consumes about $2.3 \%$ less fuel for all those selected critical mission legs, which assure the fuel efficiency of an ITB engine over the baseline engine. According to an article published in "Aerospace Engineering" magazine [1], it stated that the Continental Airlines currently flies 18 Boeing 777-200 aircrafts. If the airline could reduce the fuel consumption on that relatively small fleet of aircraft by as little as $2 \%$ per year, it would save $\$ 27$ million dollar annually. So, $2.3 \%$ fuel saved translates to a great savings in term of the operating cost.

To get an even better fuel consumption, one may want to return to the on-design cycle analysis and choose other reference-point engines for further investigation.

Table 6.2: Summary of results for mission analysis (24,000 lbf of take-off weight)

\begin{tabular}{|c|c|c|c|c|c|c|c|}
\hline & & & & Baseline & $I T B$ & & \\
\hline Mis & ion phases and segments & $M_{0}$ & Alt & Fuel used & Fuel used & Fuel saved & Fuel \\
\hline $1-2$ & A - Warm up & 0.0 & 2 & 578 & 491 & 88 & 15.1 \\
\hline $2-3$ & E - Climb/acceleration & 0.875 & 23 & 480 & 473 & 7 & 1.4 \\
\hline $3-4$ & Subsonic cruise climb & 0.8 & 42 & 505 & 501 & 9 & 1.7 \\
\hline $5-6$ & Combat air patrol & 0.697 & 30 & 705 & 700 & 5 & 0.7 \\
\hline $6-7$ & F - Acceleration & 1.09 & 30 & 221 & 223 & -2 & -0.8 \\
\hline $6-7$ & G - Supersonic penetration & 1.5 & 30 & 1819 & 1787 & 31 & 1.7 \\
\hline $7-8$ & $\mathrm{I}-1.6 \mathrm{M} / 5 \mathrm{~g}$ turn & 1.6 & 30 & 426 & 416 & 9 & 2.2 \\
\hline $7-8$ & $\mathrm{~J}-0.9 \mathrm{M} / 5 \mathrm{~g}$ turn & 0.9 & 30 & 294 & 290 & 4 & 1.2 \\
\hline $7-8$ & K - Acceleration & 1.2 & 30 & 211 & 209 & 3 & 1.3 \\
\hline $8-9$ & Escape dash & 1.5 & 30 & 531 & 523 & 8 & 1.5 \\
\hline $10-11$ & Subsonic cruise climb & 0.9 & 48 & 457 & 452 & 5 & 1.1 \\
\hline $12-13$ & Loiter & 0.378 & 10 & 608 & 612 & -3 & -0.6 \\
\hline & & & Total & 6836 & 6677 & 158 & 2.3 \\
\hline
\end{tabular}




\section{Chapter 7}

\section{Conclusions and}

\section{Recommendations}

\subsection{Conclusions}

This work presented in this dissertation is consisted of two main parts, each concerning the development of a detailed mathematical modeling to perform the parametric and performance cycle analyses, respectively. The method of analysis and the findings are first presented on each section, followed by the recommendations for future development.

\subsubsection{Parametric Cycle Analysis}

The initial task consisted of reviewing the past and current research effort on an innovative concept of introducing a secondary isobaric burner into a conventional turbofan engine, and performing a parametric cycle analysis for this modified engine. In the parametric cycle analysis, the primary purpose is to examine the variations of specific engine performance at a flight condition with changes in design parameters, including design variables for engine components. Then, it is possible to narrow down the desirable range for each de- 
sign parameter, such as the CPR, FPR, FBR, and main burner exit temperature.

The findings from the past research effort indicate the worthiness of developing ITB. However, the simplified assumptions and the absence of turbine cooling model in past researches could be over-predicting the performance gain due to the addition of ITB. Therefore, a much more detailed modeling of each engine component, including the turbine cooling model, were developed and presented in chapter 4 .

Preliminary results show that the significance of including a turbine cooling model into each computation, especially those with very high HPT inlet temperature. Consequently, turbine cooling is essential for predicting the engine performance. During the analysis of the results, a constraint in the ITB design leads to a discovery of an important quantity, namely "critical ITB temperature" $\left(T_{c i}\right)$. By definition, for a specified value of $T_{t 4.5}$, the value of $T_{c i}$ is, in fact, the maximum allowable $T_{t 4}$, beyond which the ITB has no advantage at all. In other words, whenever designing a turbofan engine with an ITB, it must always satisfy this condition: $T_{t 4}<T_{c i}$. Therefore, it is always desirable to seek for a higher $T_{c i}$ in order to utilize the advantage of ITB.

As $M_{0}$ increases, ITB engine performs better than the beseline engine at both subsonic and supersonic flights. Both ST and thermal efficiency are improved without any increase in S. At supersonic speed, the ITB engine performs even better with lesser fuel consumption. A similar conclusion can be drawn as the value of $T_{t 4}$ increases, i.e., ITB engine at supersonic flight has much improvement in thermal efficiency. Unfortunately, the overall performance of the ITB engine is only better than the baseline engine when operating at a range of $T_{t 4}$ close to $T_{c i}$.

Throughout the analysis, it is generally accepted that a high value of $T_{t 4.5}$ up 
to the turbine blade limit will give the best overall performance. However, this is not the case when the engine compression is very high (about 40 and above) at the subsonic flight. On the contrary, at the supersonic flight, ITB can be a potential improvement to the military low-FBR turbofan engine. In addition, adding ITB also widen the supersonic operation range from moderate FBR to high FBR.

Preliminary calculations also confirmed the advantage of ITB in producing lesser amount of NOx emission due to the lower peak engine temperature while maintaining same level of ST. In general, the performance comparison of the ITB engine versus the baseline engine confirmed the advantage of using ITB, i.e., higher ST, improved thermal efficiency, less cooling air, and less NOx production, provided that the values of $T_{t 4}$ and $T_{t 4.5}$ are properly specified.

Despite many advantages of using ITB for better engine performance, there are also challenges needed to be resolved. Specific hardware design challenges are the design and integration of a second combustor, including all associated cooling and control requirements, which need to be overcome.

\subsubsection{Performance Cycle Analysis}

Typically, the performance cycle analysis differs significantly from the parametric cycle analysis. Once the design choice is made in the parametric cycle analysis, it gives a design-point or reference engine for a particular application. The performance cycle analysis is then performed to estimate how this specific design-point engine will behave at conditions other than those for which it was designed. Furthermore, the performance of several design-point engines can be compared to find the most promising engine that has the best balanced performance over the entire flight envelope.

In chapter 5, a performance cycle analysis of a separate-flow and two-spool turbofan with ITB has been presented. The mathematical modeling of each 
engine component (e.g., compressors, burners, turbines and exhaust nozzles), in terms of its operating condition has been systematically described.

As expected, the results showed that the ITB engine at full throttle settings has an enhanced performance over baseline engine. Utilizing a high FBR would further reduce its fuel-burn penalty because ITB engine at high FBR has almost the same level of specific fuel consumption compared with the baseline engine.

At the partial throttle settings, adding ITB further extends the engine operational range by producing higher thrust levels than that of the baseline engine. Within these higher thrust curves, the fuel consumption increases linearly with increasing thrust until it reaches their full throttle settings. On the other hand, ITB engine at partial throttle settings achieves a modest thrust augmentation without any fuel-burn penalty as observed in most full throttle settings. This advantage greatly provide fuel saving to many aircraft engines, which normally run at partial load during the cruise operations at high altitude.

A systematic mission study of the fuel consumption is performed to reveal the advantage of saving fuel by adding ITB. ITB engine uses less fuel in each critical mission phase. On an average, the calculation shows that ITB engine consumes about $2.6 \%$ less fuel for all those selected critical mission legs, which assures the ITB engine's advantage of saving fuel over the baseline engine.

\subsection{Recommendations}

Although the program developed in this work is considered a fairly complete and fully functional design tool for the research on ITB, it is possible to identify four main directions for future work. All these suggestions are recommended so that the program could be enhanced for increased usefulness. 


\subsubsection{Increased Flexibility of the Program}

The two Excel programs, i.e., for parametric and performance cycle analysis, respectively, have been written with a specific engine configuration in mind, i.e., namely an unmixed two-spool turbofan with separate fan and core stream nozzles. Applications of an ITB may not be limited to a given configuration. In fact, the addition of an ITB may foster unique engine configurations that can better leverage the increased specific thrust. As a result, an increased flexibility of engine configuration would be a desirable feature.

One example would be the mixed turbofan that in most cases employ a forced mixer at the mixing plane, where the fan and the core streams merge together. To accomplish this capability, some branching of the source code can be done to allow for a different set of equations, like adding another independent fan bypass ratio along with a dependent for the mixing plane static pressure balance and some mixed nozzle performance calculations. Parameters such as thermal mixing and pressure losses in the mixer can also be included to characterize the mixer performance.

In the findings of Liu and Sirignano [32] and Chen et al. [4], they both found out the superior advantage of operating two ITBs, instead of one ITB. To further investigate the potential of the 2-ITB engine, it may require a three-spool arrangement type of turbofan, such as the family of high-bypass and triple-spool Rolls-Royce Trent engines. Therefore, it will be an enhanced feature if this kind of three-spool engine configuration is incorporated into the program.

\subsubsection{Multi-stage Turbine and Cooling Model}

In this research, the HPT and LPT are both modeled as single-stage turbine for simplicity. Accordingly, the turbine cooling scheme is modeled as two injections at the entrances of the first stator and rotor separately. While this method 
provides certain level of accuracy for most engines, the simple assumption may not be valid when designing a multi-stage turbine. In a multi-stage turbine, the increased power could be used to drive more engine compressions.

In order to accurately predict the behavior of the multi-stage turbine in future work, the requirement for a more detailed modeling of cooling model at each turbine stage is also a must. This would likely be a branch in the code that is tied to the modeling of alternate multi-stage turbine. For example, Kesser [19] has successfully adopted a perfect mixer cooling flow model in his work of analyzing a basic chemically recuperated gas turbine power plant.

\subsubsection{Design Choice Optimization}

As reader observed the curve of 'S versus FPR' at a subsonic flight (Figure 6.9), there is an optimum value of the FPR that leads to minimum thrust specific

fuel consumption. For a given set of prescribed variables $\left(e . g ., \pi_{c}, \pi_{f}, T_{t 4}, M_{0}\right)$, one can locate the minimum $\mathrm{S}$ by taking the partial derivative of $\mathrm{S}$ with respect to the FPR. Similar techniques can be applied to other design choices, for instance, the FBR at supersonic flight (Figure 6.12). Although this kind of task looks simple, however, it is in fact a tedious mathematical formulation problem, which requires a sound mathematical background in the field of design optimization. A good example can be found in chapter 7 of Mattingly's textbook [33].

\subsubsection{Separate Modeling of Fan Hub and Tip}

In this research, the fan was treated on an overall basis for both bypass and core stream flows. On a close coupled fan configuration, like most of the fighter aircraft engines, fan performance is broken down so that the hub and tip performance can be characterized independently. Therefore, having this capability to treat the fan hub and tip separately in the future code development would be a useful feature too. 
APPENDICES 


\section{Appendix A}

\section{Summary of Papers}

\section{A Complete Parametric Cycle Analysis of a Turbofan with Interstage Turbine Burner (AIAA-2003-0685) [25]}

Today's modern aircraft is based on air-breathing jet propulsion systems, which use moving fluids as substances to transform energy carried by the fluids into power. Throughout aero-vehicle evolution, improvements have been made to the engine efficiency and pollutants reduction. This study focuses on a parametric cycle analysis of a dual-spool, separate-flow turbofan engine with an ITB. The ITB considered in this paper is a relatively new concept in modern jet engine propulsion. The ITB serves as a secondary combustor and is located between the high- and the low-pressure turbine, i.e., the transition duct. The objective of this study is to use design parameters, such as flight Mach number, compressor pressure ratio, fan pressure ratio, fan bypass ratio, linear relation between highand low-pressure turbines, and high-pressure turbine inlet temperature to obtain engine performance parameters, such as specific thrust and thrust specific fuel consumption. Results of this study can provide guidance in identifying the performance characteristics of various engine components, which can then be used to develop, analyze, integrate, and optimize the system performance of turbofan engines with an ITB.

\section{Performance Cycle Analysis of a Two-spool Separate-exhaust Turbofan with Interstage Turbine Burner (AIAA-2004-3311) [26]}

This paper presents the performance cycle analysis of a dual-spool, separateexhaust turbofan engine, with an Interstage Turbine Burner serving as a secondary combustor. A performance analysis of this engine has been conducted for steady-state engine performance prediction. A code is written and is capable of predicting engine performances (i.e. thrust and thrust specific fuel consumption) at varying flight conditions and throttle settings. Two design point engines were studied to reveal trends in performance at full and partial throttle operations. 


\section{An Interactive Microsoft Excel program for Tracking A Single Evapo- rating Droplet in Crossflow (NASA/TM-2004-212910) [27]}

Droplet interaction with a high temperature gaseous crossflow is important because of its wide application in systems involving two phase mixing such as in combustion requiring quick mixing of fuel and air. The focus of this work is to investigate dispersion of a two-dimensional evaporating spray into a crossflow.

An interactive Microsoft Excel program for tracking a single droplet in crossflow that has previously been developed was modified to include droplet evaporation computation. In addition to the high velocity airflow, the injected droplets are also subjected to increased combustor temperature and pressure that affect their motion in the flow field. Six ordinary differential equations (namely the time rate of change of $x, z, u_{d}, w_{d}, D$, and $T_{s}$ ) are then solved by $4^{\text {th }}$-order Runge-Kutta method using Microsoft Excel software.

Visual Basic programming and Excel macrocode are used to calculate the data and plot the droplet's motion in the flow field. This program computes and plots the data sequentially without forcing the user to open other types of plotting programs. A user's manual on how to use the program is included.

\section{Parametric Cycle Analysis of a Turbofan with Interstage Turbine Burner 28]}

The objective of this study is to use engine design parameters, such as highpressure and low-pressure turbine inlet temperatures to obtain engine performance parameters, e.g., specific thrust and thrust specific fuel consumption. A turbine cooling model is also included. Results confirm the advantages of ITB, i.e., higher ST, less cooling air, and possibly less NOx production, provided that the main burner exit temperature and ITB exit temperature are properly specified.

\section{A Parametric (On-Design) Cycle Analysis for a Separate-Exhaust Turbofan Engine with Interstage Turbine Burner (NASA/TM-2005- 213658) 29.}

The objective of this study is to use design parameters, such as flight Mach number, compressor pressure ratio, fan pressure ratio, fan bypass ratio, and high-pressure turbine inlet temperature to obtain engine performance parameters, such as specific thrust and thrust specific fuel consumption. Results of this study can provide guidance in identifying the performance characteristics of various engine components, which can then be used to develop, analyze, integrate, and optimize the system performance of turbofan engines with an ITB.

Visual Basic program, Excel macrocode, and Excel neuron code are used to facilitate Excel software to plot engine performance versus engine design param- 
eters. This program computes and plots the data sequentially without forcing users to open other types of plotting programs. A user's manual on how to use the program is also included in this report. Furthermore, this stand-alone program is written in conjunction with an off-design program which is an extension of this study. The computed result of a selected design-point engine will be exported to an engine reference data file that is required in off-design calculation.

\section{Performance (Off-Design) Cycle Analysis for a Turbofan Engine with Interstage Turbine Burner (NASA/TM-2005-213659) [30]}

A detailed off-design performance analysis of ITB engines is written in Excel macrocode with Visual Basic Application to calculate engine performances over the entire operating envelope. Several design-point engine cases are pre-selected using a parametric cycle analysis code developed previously in Excel, for offdesign analysis. The off-design code calculates engine performances (i.e. thrust and thrust-specific-fuel-consumption) at various flight conditions and throttle settings.

\section{Performance Cycle Analysis of Turbofan Engine with Interstage Tur- bine Burner [31]}

This paper presents the performance cycle analysis of a dual-spool, separateexhaust turbofan engine, with an Interstage Turbine Burner serving as a secondary combustor. The ITB, which is located at the transition duct between the high- and the low-pressure turbines, is a relatively new concept for increasing specific thrust and lowering pollutant emissions in modern jet engine propulsion. A performance analysis of this engine has been conducted for steady-state engine performance prediction. A code is written and is capable of predicting engine performances (i.e., thrust and thrust specific fuel consumption) at varying flight conditions and throttle settings. Two design-point engines were studied to reveal trends in performance at both full and partial throttle operations. A mission analysis is also presented to assure the advantage of saving fuel by adding ITB. 


\section{Appendix B}

\section{U.S. Standard Atmosphere 1976}

\section{B.1 Introduction}

The US Standard Atmosphere 1976 is an average, piece-wise continuous function for density, pressure and temperature profiles of the earth's standard atmosphere. The standard atmosphere is mathematically defined in seven layers from sea level to $84.852 \mathrm{~km}$. It is a function of geo-potential altitude, which is represented by

$$
z=\frac{h \cdot R_{\text {earth }}}{h+R_{\text {earth }}}
$$

where $h$ is the geometric altitude (unit:km) and $R_{\text {earth }}$ is the radius of the earth $(=6356.66 \mathrm{~km})$.

The table B.1 summarizes the values of several parameters at each of the defined levels.

\section{B.2 Temperature}

The variation of temperature, $T(z)$, with geo-potential altitude is expressed by a continuous and piecewise linear relation. If Lapse rate is non-zero $\left(L R_{s t d, i} \neq\right.$ 
$0)$,

$$
T(z)=T_{s t d, i}+L R_{s t d, i} \cdot\left(z-z_{s t d, i}\right)
$$

Table B.1: Values of several parameters at each defined levels of the earth's atmosphere.

\begin{tabular}{|l|l|l|l|l|l|l|}
\hline $\begin{array}{l}\text { Layer } \\
(i)\end{array}$ & Name & $\begin{array}{l}\text { Lower } \\
\text { Geopotential } \\
\text { Altitude }\left(z_{s t d, i}\right)\end{array}$ & $\begin{array}{l}\text { Upper } \\
\text { Geopotential } \\
\text { Altitude }\end{array}$ & $\begin{array}{l}\text { Lapse } \\
\text { rate } \\
\left(L R_{s t d, i}\right)\end{array}$ & $\begin{array}{l}\text { Standard } \\
\text { Pressure, } \\
\left(P_{\text {std }, i}\right)\end{array}$ & $\begin{array}{l}\text { Standard } \\
\text { Temperature, } \\
\left(T_{s t d, i}\right)\end{array}$ \\
\hline & Unit & $\mathbf{k m}$ & $\mathbf{k m}$ & $\mathbf{K} / \mathbf{k m}$ & $\mathbf{P a}$ & $\mathbf{K}$ \\
\hline 1 & Troposphere & 0 & 11 & -6.5 & 101325 & 288.15 \\
\hline 2 & Stratosphere & 11 & 20 & 0.0 & 22632.06 & 216.65 \\
\hline 3 & - & 20 & 32 & +1.0 & 5474.89 & 216.65 \\
\hline 4 & - & 32 & 47 & +2.8 & 868.02 & 228.65 \\
\hline 5 & - & 47 & 51 & 0.0 & 110.91 & 270.65 \\
\hline 6 & Mesosphere & 51 & 71 & -2.8 & 66.94 & 270.65 \\
\hline 7 & - & 71 & 84.852 & -2.0 & 3.96 & 214.65 \\
\hline
\end{tabular}

If Lapse rate is zero $\left(L R_{s t d, i}=0\right)$,

$$
T(z)=T_{s t d, i}
$$

\section{B.3 Pressure}

The pressure, $P(z)$, is also a continuous piecewise function of geo-potential altitude. If Lapse rate

$$
P(z)=P_{s t d, i}\left(\frac{T_{s t d}(z)}{T_{s t d, i}}\right)^{\left(\frac{-1000 \cdot g_{0} \cdot W_{0}}{R^{*} \cdot L R_{s t d, i}}\right)}
$$

If Lapse rate is zero $\left(L R_{s t d, i}=0\right)$, 


$$
P(z)=P_{s t d, i} \cdot \exp \left(\frac{-1000 \cdot g_{0} \cdot W_{0} \cdot\left(z-z_{s t d, i}\right)}{R^{*} \cdot T_{s t d, i}}\right)
$$

where $g$ is the acceleration of gravity $\left(=9.80665 \mathrm{~m} / \mathrm{s}^{2}\right), R^{*}$ is the gas constant for air $(=8314.32 \mathrm{~J} / \mathrm{kmol}-\mathrm{K}), W_{0}$ is the molecular weight of air $(=28.9644$ $\mathrm{kg} / \mathrm{kmol})$. 


\section{Appendix C}

\section{Combustion Fuel Burn Model}

\section{C.1 Combustion Fuel Burn Models}

Two models are being used to calculate the amount of fuel burned in the main burner and the ITB in terms of the fuel-air ratio. [34]:

1. Constant Specific Heat (CSH) model

The air and combustion gases at inlet and exit of each component are modeled as "calorically perfect gases" with constant specific heats. The values of the specific heats are different at inlet and exit of two combustors (main burner and ITB)

2. Modified Specific Heat (MSH) model

All engines properties are calculated using CSH model except the fuel used. In this model, the inlet total temperature (i.e., $T_{t 3}$ and $T_{t 4.4}$ ) of combustors are calculated using CSH model while the exit total temperatures $\left(T_{t 4}\right.$ and $\left.T_{t 4.5}\right)$ are directly obtained from the user inputs. Nevertheless, the total enthalpies $\left(h_{t 3}, h_{t 4}, h_{t 4.4}\right.$, and $\left.h_{t 4.5}\right)$ will be calculated directly from the Variable Specific Heat (VSH) model (p.116 of [34]). Therefore, the improvement on enthalpy calculation gives better estimates of fuel used. 


\section{C.2 ENTHALPY Subroutine}

The following ENTHALPY subroutine is based on pages 105-106 of [33] and used in the MSH model to calculate the enthalpy $(h)$ at engine stations $3,4,4.4$, and 4.5 .

ENTHALPY(option, $\left.T, f, h, c_{p}, \gamma\right)$

Inputs: $T$ and $f$

Outputs: $h$, or $\left(c_{p}\right.$ and $\left.\gamma\right)$

The equations for calculating these outputs are shown below:

$h_{\text {air }}=\left[h_{\text {ref }}+A_{0} T+\frac{A_{1}}{2} T^{2}+\frac{A_{2}}{3} T^{3}+\frac{A_{3}}{4} T^{4}+\frac{A_{4}}{5} T^{5}+\frac{A_{5}}{6} T^{6}+\frac{A_{6}}{7} T^{7}+\frac{A_{7}}{8} T^{8}\right]_{\text {air }}$

$$
\begin{gathered}
h_{\text {prod }}=\left[h_{\text {ref }}+A_{0} T+\frac{A_{1}}{2} T^{2}+\frac{A_{2}}{3} T^{3}+\frac{A_{3}}{4} T^{4}+\frac{A_{4}}{5} T^{5}+\frac{A_{5}}{6} T^{6}+\frac{A_{6}}{7} T^{7}+\frac{A_{7}}{8} T^{8}\right]_{\text {prod }} \\
\text { (C.2) }
\end{gathered}
$$

where the constants $A_{0}$ etc. are given in Table C.1.

The equations needed to calculate the fuel-air ratios of the main burner $\left(f_{b}\right)$ and the ITB $\left(f_{i t b}\right)$ are listed below for both the CSH and MSH models.

\section{Main burner}

If $(\mathbf{C S H})$ then $(\mathrm{Eq}, 4.13)$

$$
f_{b}=\frac{\tau_{\lambda-b}-\tau_{r} \tau_{d} \tau_{c L} \tau_{c H}}{\eta_{b} h_{P R} /\left(c_{p c} T_{0}\right)-\tau_{\lambda-b}}
$$

Else

$$
T_{t 3}=T_{0} \tau_{r} \tau_{c L} \tau_{c H}
$$


Table C.1: Constants for air and combustion products used in subroutine ENTHALPY(option, $\left.T, f, h, C_{p}, \gamma\right)$.

\begin{tabular}{|c|c|c|}
\hline $\begin{array}{c}\text { Constant } \\
A_{0}\end{array}$ & Air alone & Combustion products of air and $\left(\mathrm{CH}_{2}\right)_{n}$ fuels \\
\hline$A_{1}$ & $-5.153620051 \times 10^{-1}$ & $7.3816638 \times 10^{-1}$ \\
\hline$A_{2}$ & $6.5519486 \times 10^{-5}$ & $1.2258630 \times 10^{-5}$ \\
\hline$A_{3}$ & $-6.7178376 \times 10^{-12}$ & $-1.3771902 \times 10^{-8}$ \\
\hline$A_{4}$ & $-1.5128259 \times 10^{-14}$ & $9.9686793 \times 10^{-12}$ \\
\hline$A_{5}$ & $7.6215767 \times 10^{-18}$ & $-4.2051104 \times 10^{-14}$ \\
\hline$A_{6}$ & $-1.4526770 \times 10^{-21}$ & $1.0212913 \times 10^{-18}$ \\
\hline$A_{7}$ & $1.0115540 \times 10^{-25}$ & $-1.3335668 \times 10^{-21}$ \\
\hline$h_{r e f}$ & $-1.7558886 \mathrm{Btu} / \mathrm{lbm}$ & $3.2678710 \times 10^{-25}$ \\
\hline
\end{tabular}

$\operatorname{ENTHALPY}\left(0, T_{t 3}, 0, h_{t 3}, 0,0\right)$

Set initial value of fuel/air ratio at station 4 equal to $f_{4 i}$.

$3 \quad \boldsymbol{E N T H A L P Y}\left(0, T_{t 4}, f_{4 i}, h_{t 4}, 0,0\right)$

$$
f_{b}=\frac{h_{t 4}-h_{t 3}}{\eta_{b} h_{P R}-h_{t 4}}
$$

If $\left|f_{b}-f_{4 i}\right|>0.0001$, then $f_{4 i}=f_{b}$ and go to 3 ; else continue.

Endif

Interstage Turbine Burner

If $(\mathbf{C S H})$ then (Eq.4.16)

$$
f_{i t b}=\frac{\tau_{\lambda-i t b}-\tau_{\lambda-b} \tau_{m 1} \tau_{m 1} \tau_{t H}}{\eta_{i t b} h_{P R} /\left(c_{p c} T_{0}\right)-\tau_{\lambda-i t b}}
$$

Else

$$
T_{t 4.4}=T_{t 4} \tau_{t H}
$$

$\operatorname{ENTHALPY}\left(0, T_{t 4.4}, f_{b}, h_{t 4.4}, 0,0\right)$

Set initial value of fuel/air ratio at station 4.4 equal to $f_{4.5 i}$.

$4 \quad \operatorname{ENTHALPY}\left(0, T_{t 4.5}, f_{4.5 i}, h_{t 4.5}, 0,0\right)$

$$
f_{i t b}=\frac{h_{t 4.5}-h_{t 4.4}}{\eta_{i t b} h_{P R}-h_{t 4.5}}
$$


If $\left|f_{b}+f_{i t b}-f_{4.5 i}\right|>0.0001$, then $f_{4.5 i}=f_{b}+f_{i t b}$ and go to 4 ; else continue.

Endif

If $f_{i t b}=0$ then $\gamma_{i t b}=\gamma_{t}, c_{p i t b}=c_{p t}$, and $R_{i t b}=R_{t}$.

\section{C.3 Products of Equilibrium Combustion}

An additional subroutine is included to give the values of molar fractions for each equilibrium combustion product. These computed equilibrium products of combustion for a fuel composed of $\mathrm{C}, \mathrm{H}, \mathrm{O}$, and $\mathrm{N}$ atoms are required as the inputs for the prediction of $N O_{x}$ in the post-flame zone.

\section{C.3.1 Olikara and Borman model}

The solution procedure for the calculation of equilibrium combustion is based on the approach first developed by Olikara and Borman [40]. Their code, written in FORTRAN language, solves a global fuel oxidation reaction involving 13 species, including the hydrocarbon fuel (i.e., $\mathrm{C}_{n} \mathrm{H}_{m} \mathrm{O}_{l} \mathrm{~N}_{k}$ ), $\mathrm{O}_{2}, \mathrm{~N}_{2}, \mathrm{H}, \mathrm{O}, \mathrm{N}, \mathrm{OH}$, $\mathrm{CO}, \mathrm{NO}, \mathrm{CO}_{2}, \mathrm{H}_{2} \mathrm{O}$, and $\mathrm{Ar}$ :

$$
\begin{aligned}
x_{13} & {\left[C_{n} H_{m} O_{l} N_{k}+\frac{n+m / 4-l / 2}{\phi}\left(O_{2}+3.7274 N_{2}+0.0444 A r\right)\right] \longrightarrow x_{1} H+x_{2} O+x_{3} N } \\
& +x_{4} H_{2}+x_{5} O H+x_{6} C O+x_{7} N O+x_{8} O_{2}+x_{9} H_{2} O+x_{10} C_{2}+x_{11} N_{2}+x_{12} A r
\end{aligned}
$$

For simplicity, the mole fraction of inert gas $A r, x_{12}$, is always set to 0 during the computation.

In addition, the code also invokes the following 7 equilibrium reactions:

$$
\frac{1}{2} H_{2} \rightleftharpoons H
$$




$$
\begin{gathered}
\frac{1}{2} \mathrm{O}_{2} \rightleftharpoons \mathrm{O} \\
\frac{1}{2} \mathrm{~N}_{2} \rightleftharpoons \mathrm{N} \\
\frac{1}{2} \mathrm{H}_{2}+\frac{1}{2} \mathrm{O}_{2} \rightleftharpoons \mathrm{OH} \\
\frac{1}{2} \mathrm{O}_{2}+\frac{1}{2} \mathrm{~N}_{2} \rightleftharpoons \mathrm{NO} \\
\mathrm{H}_{2}+\frac{1}{2} \mathrm{O}_{2} \rightleftharpoons \mathrm{H}_{2} \mathrm{O} \\
\mathrm{CO}+\frac{1}{2} \mathrm{O}_{2} \rightleftharpoons \mathrm{CO}_{2}
\end{gathered}
$$

The equilibrium constants are curved fitted from data in JANAF thermochemical table [18]. For a much more complete description of Olikara and Borman's model and its example calculations, please refer to [40].

\section{C.3.2 Adaptation to Gas Turbine Combustor and its lim- itations}

Although this code was developed specifically for automotive internal combustion engine simulations, it can also be used for gas turbine combustor simulations provided the pressure and temperature inside the combustor and the chemical formulation for kerosene or Jet-A are properly specified. In this study, either kerosene or Jet-A is used as a fuel and both formulations are specified as $C_{12} H_{23}$ [20], where $n=12, m=23, l=0$, and $k=0$.

Reader needs to be aware of the limitations for this model. One of them is that the products of combustion are assumed to be ideal gases. This assumption is not valid at extremely high pressures, similar to those encountered inside a gas turbine combustor. Secondly, due to the short residence time, it is impossible for the combustion products to attain equilibrium. Despite all these limitations, it is at least an ideal way to calculate the mole fractions of combustion products, 
which are required as the inputs in the prediction of $N O_{x}$ formation.

\section{C.4 NOx model}

In order to provide more information to assess the effect of adding ITB on the engine performance, it is important to provide information on the pollutant emissions, particularly the $N O_{x}$ emission in this study. As a result, a separate subroutine has been embedded into the combustion submodel to predict $N O_{x}$ production.

\section{C.4.1 Oxides of Nitrogen mechanisms}

Of the oxides of nitrogen $\left(N O_{x}\right)$ generated by gas turbine combustor, $N O$ occupies more than $90 \%$ of the $N O_{x}$ [43. Therefore, only the production of $N O$ is modeled. In the combustion of fuels that contain no nitrogen, $N O$ is formed by three chemical mechanisms that involve nitrogen in the air: (1) the thermal

or Zeldovich mechanism, (2) the prompt or Fenimore mechanism, and (3) the $\mathrm{N}_{2} \mathrm{O}$-intermediate mechanism [51].

The prompt mechanism is of no interest in this study because it is only important in low-temperature fuel-rich zone [21]. Meanwhile, the $\mathrm{N}_{2} \mathrm{O}$-intermediate mechanism is only important in lean premixed combustion and is not well developed yet [51]. Consequently, it is reasonable to neglect the prompt and $\mathrm{N}_{2} \mathrm{O}$ intermediate mechanisms. Only the fairly well established Zeldovich chain mechanism or simply the thermal mechanism is applied to predict the NO formation. This assumption is valid for most traditional combustors.

\section{C.4.2 Extended Zeldovich Mechanism}

The amount of thermal NO produced is computed using the extended Zeldovich mechanism is shown as follows [51]: 


$$
\begin{gathered}
N_{2}+O \rightleftharpoons N O+N \\
O_{2}+N \rightleftharpoons N O+O \\
O H+N \rightleftharpoons N O+H
\end{gathered}
$$

The rate coefficients for equations eqs. C.18 to C.20 (unit: $\mathrm{m}^{3} / \mathrm{kmol}-\mathrm{s}$ ) are 12

$$
\begin{gathered}
k_{f 1}=1.8 \cdot 10^{11} \exp [-38370 / T] \\
k_{f 2}=1.8 \cdot 10^{7} \operatorname{Texp}[-4680 / T] \\
k_{f 3}=7.1 \cdot 10^{10} \exp [-450 / T] \\
k_{b 1}=3.8 \cdot 10^{10} \exp [-425 / T] \\
k_{b 2}=3.8 \cdot 10^{6} \mathrm{Texp}[-20820 / T] \\
k_{b 3}=1.7 \cdot 10^{11} \exp [-24560 / T]
\end{gathered}
$$

This formation rate of $N O$ (unit: $\mathrm{kmol} / \mathrm{m}^{3}-\mathrm{s}$ ) can be expressed as

$$
\begin{aligned}
\frac{d[N O]}{d t}= & k_{f 1}[O]\left[N_{2}\right]+k_{f 2}[N]\left[O_{2}\right]+k_{f 3}[N][O H]-k_{b 1}[N][N O] \\
& -k_{b 2}[O][N O]-k_{b 3}[H][N O]
\end{aligned}
$$

where $[N O]$ denotes the molar concentration (unit: $\mathrm{kmol} / \mathrm{m}^{3}$ ).

\section{C.4.3 Assumptions and Limitations}

In general, the $N O$ mechanism above can be decoupled from the fuel oxidation computation because the $N O$ formation becomes significant only after the fuel oxidation is complete. Despite the fact that the residence time is short, approximately 1 to $3 \mathrm{msec}$ [44], it is assumed here that the $\mathrm{N}_{2}, \mathrm{O}_{2}, \mathrm{O}, \mathrm{H}$, and $\mathrm{OH}$ concentrations are at their equilibrium values and $N$ atom is in steady state. This assumption greatly simplifies the computation of $N O$ formation. 


\section{C.4.4 Solution Procedure}

The following shows a systematic solution procedure for the computation of emission index for $N O, E I_{N O}$ (unit: $g / k g$ ):

1. Compute the mole fractions of these species, $\chi_{i}$, including $N_{2}, O_{2}, O, H$, and $O H$ from the equilibrium combustion [from section C.3].

2. Determine the total number of mole of combustion products $\left(n_{p}\right)$ using 'Equation of State':

$$
P V=n_{p} R T \Longrightarrow \frac{n_{p}}{V}=\frac{P}{R T}
$$

Then, convert the mole fraction of each species to molar concentration:

$$
\left[\text { species }_{i}\right]=\chi_{i} \times \frac{n_{p}}{V}
$$

3. Evaluate the rate coefficients from eqs. (C.21) to (C.26).

4. Solve the ordinary differential equation (C.27) using Runge Kutta method [38]:

Assume the concentrations of $\mathrm{N}_{2}, \mathrm{O}_{2}, \mathrm{O}, \mathrm{H}, \mathrm{OH}$, and $\mathrm{N}$ are at their equilibrium values and stay constant, eq. (C.27) can be expressed as

$$
\frac{d[N O]}{d t}=C_{f}-C_{b}[N O]=f([N O])
$$

where $C_{f}=k_{f 1}[O]\left[N_{2}\right]+k_{f 2}[N]\left[O_{2}\right]+k_{f 3}[N][O H]$

$$
C_{b}=k_{b 1}[N]-k_{b 2}[O]-k_{b 3}[H]
$$

Through the Runge Kutta numerical method, the solution is thus

$$
[N O]_{n+1}=[N O]_{n}+\frac{1}{6}\left(n o_{1}+2 n o_{2}+2 n o_{3}+n o_{4}\right)
$$


where

$$
\begin{aligned}
& n o_{1}=\Delta t \cdot f\left([N O]_{n}\right) \\
& n o_{2}=\Delta t \cdot f\left([N O]_{n}+\frac{n o_{1}}{2}\right) \\
& n o_{3}=\Delta t \cdot f\left([N O]_{n}+\frac{n o_{2}}{2}\right) \\
& n o_{4}=\Delta t \cdot f\left([N O]_{n}+n o_{3}\right)
\end{aligned}
$$

\section{C.4.5 Comparison to Empirical NOx Correlations}

Lefebvre [22] did an excellent job in correlating a great amount of experimental data of $N O_{x}$ measurement, which cover a wide range of fuel types from JP4 to DF2. The predicted $N O_{x}$ data using the correlation equation (C.33) shown below has an excellent agreement with the experimental data for almost all combustors. Therefore, it is reasonable to compare our predicted $N O_{x}$ data to Lefebvre's data calculated using his empirical equation:

$$
\left[N O_{x}\right]=\frac{9 \times 10^{-8} P_{3}^{1.25} V_{c} \exp \left(0.01 T_{s t}\right)}{\dot{m}_{c} T_{p z}} \quad\left[\text { unit }: \frac{g}{\mathrm{~kg}}\right]
$$

where $\quad V_{c}$ is the total combustion volume $\left(\mathrm{m}^{3}\right)$,

$T_{s t}$ is the stoichiometric flame temperature,

$T_{p z}$ represents the relationship $T_{3}+\Delta T_{p z}$,

$\Delta T_{p s}$ is the temperature rise due to combustion corresponding to $T_{3}$,

$\dot{m}_{c}$ is the air mass flow rate.

Again, reader should be aware of the purpose of the simple $N O_{x}$ model, which is to give us a qualitative assessment of the effect of adding an ITB on the engine performance. Accordingly, emphasis has been put into predicting the trends in $N O_{x}$ emission, not into calculating an accurate quantitative value of the emission. Then, reader should not be surprised to observe the discrepancy between the simple Zeldovich model and Lefebvre's model as shown in Figure C.1. 


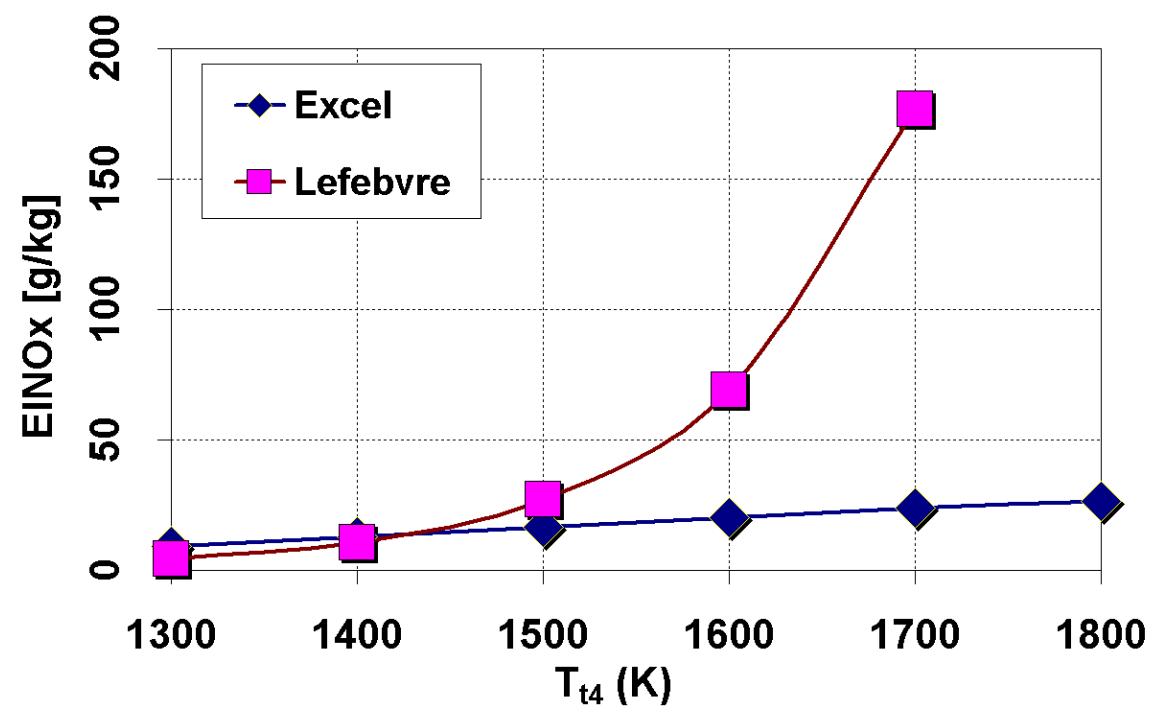

Figure C.1: Comparison of Predicted NOx Data between the simple Zeldovich model and Lefebvre's empirical model

According to Figure C.1, the predicted trends by both models obviously follow a logical behavior, where $N O_{x}$ level increases as the combustor temperature increases. 


\section{Appendix D}

\section{Summary of Equations for Parametric Cycle Analysis}

Appendix D summarizes the complete parametric (or on-design) cycle analysis equations for the separate-exhaust, two-spool turbofan engine with an ITB and turbine cooling. The equations shown below follows the order of the solution in the computer code. This solution procedure requires no iteration.

\section{D.1 Inputs}

Flight parameters:

Design choices:

Design limitations:

Aircraft system parameter:

Component figures of merit: $\pi_{d \max }, \pi_{b}, \pi_{i t b}, \pi_{n}, \pi_{n f}$

$e_{c H}, e_{c L}, e_{t L}, e_{t H}, e_{f}$

$\eta_{b}, \eta_{i t b}, \eta_{m H}, \eta_{m L}$

Fuel heating value:

$M_{0}, h, T_{0}, P_{0}$

$\pi_{c}, \pi_{c L}, \pi_{f}, \alpha$

$T_{t 4}, T_{t 4.5}$

$h_{P R}$
$P_{0} / P_{9}, P_{0} / P_{19}$ (for convergent-divergent nozzle only) 


\section{D.2 Outputs}

Overall performance: $F / \dot{m}_{0}, S, \eta_{t h}, \eta_{P}, \eta_{O}, V_{9} / a_{0}, V_{19} / a_{0}$

Component behavior: $f_{o}, f_{b}, f_{i t b}, \tau_{\lambda-b}, \tau_{\lambda-i t b}$

$$
\begin{aligned}
& M_{8}, M_{9}, M_{18}, M_{19}, \epsilon_{1}, \epsilon_{2} \\
& \tau_{t H}, \tau_{t L}, \tau_{c H}, \tau_{c L}, \tau_{f}, \pi_{t H}, \pi_{t L}, \pi_{c H}, \pi_{c L}, \pi_{f} \\
& \eta_{t H}, \eta_{t L}, \eta_{c H}, \eta_{c L}, \eta_{f}
\end{aligned}
$$

\section{D.3 Equations}

$$
\begin{aligned}
R_{c} & =\frac{\gamma_{c}-1}{\gamma_{c}} c_{p c} \\
R_{t} & =\frac{\gamma_{t}-1}{\gamma_{t}} c_{p t} \\
a_{0} & =\sqrt{\gamma_{c} R_{c} g_{c} T_{0}} \\
V_{0} & =M_{0} a_{0}
\end{aligned}
$$

\section{Diffuser}

$$
\begin{aligned}
& \tau_{r}=1+\frac{\gamma_{c}-1}{2} M_{0}^{2} \\
& \pi_{r}=\tau_{r}^{\gamma_{c} /\left(\gamma_{c}-1\right)} \\
& \eta_{R \text { spec }}= \begin{cases}1 & \text { for } M_{0} \leq 1 \\
1-0.075\left(M_{0}-1\right)^{1.35} & \text { for } 1<M_{0}<5 \\
\frac{800}{M_{0}^{4}+935} & \text { for } M_{0}>5\end{cases} \\
& \pi_{d}=\pi_{d \operatorname{axax}} \eta_{R s p e c} \\
& \tau_{\lambda-b}=\frac{c_{p t} T_{t 4}}{c_{p c} T_{0}}
\end{aligned}
$$

\section{Compressors and Fan}

$$
\begin{aligned}
& \tau_{c H}=\pi_{c H}^{\left(\gamma_{c}-1\right) /\left(\gamma_{c} e_{c H}\right)} \\
& \tau_{c L}=\pi_{c L}^{\left(\gamma_{c}-1\right) /\left(\gamma_{c} e_{c L}\right)} \\
& \tau_{f}=\pi_{f}^{\left(\gamma_{c}-1\right) /\left(\gamma_{c} e_{f}\right)} \\
& \tau_{c}=\tau_{c L} \tau_{c H}
\end{aligned}
$$




$$
\begin{aligned}
& \tau_{b}=\frac{T_{t 4}}{T_{0} \tau_{r} \tau_{c}} \\
& \eta_{c H}=\frac{\tau_{c H}^{e_{c H}}-1}{\tau_{c H}-1} \\
& \eta_{c L}=\frac{\tau_{c L}^{c L}-1}{\tau_{c L}-1} \\
& \eta_{f}=\frac{\tau_{f}^{e}-1}{\tau_{f}-1}
\end{aligned}
$$

\section{Main Burner}

If $(\mathbf{C S H})$ model then $f_{b}=\frac{\tau_{\lambda-b}-\tau_{r} \tau_{c L} \tau_{c H}}{\eta_{b} h_{P R} /\left(c_{p c} T_{0}\right)-\tau_{\lambda-b}}$

Else

$$
T_{t 3}=T_{0} \tau_{r} \tau_{c L} \tau_{c H}
$$

$\operatorname{ENTHALPY}\left(0, T_{t 3}, 0, h_{t 3}, 0,0\right)$

Set initial values of fuel/air ratio at station $4, f_{4 i}=\frac{\tau_{\lambda-b}-\tau_{r} \tau_{c L} \tau_{c H}}{\eta_{b} h_{P R} /\left(c_{p c} T_{0}\right)-\tau_{\lambda-b}}$

3

$\operatorname{ENTHALPY~}\left(0, T_{t 4}, f_{4 i}, h_{t} 4,0,0\right) f_{b}=\frac{h_{t 4}-h_{t 3}}{\eta_{b} h_{P R}-h_{t 4}}$

If $\left(\left|f_{b}-f_{4 i}\right|>0.0001\right)$, then $f_{4 i}=f_{b}$ and goto 3 ; Else continue.

Endif

\section{High-Pressure Turbine}

$$
\operatorname{COOLING}\left(T_{t 4}, \tau_{c}, \tau_{\lambda-b}, \beta, \epsilon_{1}, \epsilon_{2}\right)
$$

$\tau_{m 1}=\frac{\left(1-\beta-\epsilon_{1}-\epsilon_{2}\right)\left(1+f_{b}\right)+\epsilon_{1} \tau_{r} \tau_{d} \tau_{c} / \tau_{\lambda-b}}{\left(1-\beta-\epsilon_{1}-\epsilon_{2}\right)\left(1+f_{b}\right)+\epsilon_{1}}$

$\tau_{t H}=1-\frac{\left(\tau_{c H}-1\right) \tau_{c L} \tau_{r}}{\left[\left(1-\beta-\epsilon_{1}-\epsilon_{2}\right)\left(1+f_{b}\right)+\epsilon_{1}\right] \tau_{\lambda-b} \tau_{m 1} \eta_{m H}}$

$\pi_{t H}=\tau_{t H}^{\gamma_{t} /\left[\left(\gamma_{t}-1\right) e_{t H}\right]}$

$\tau_{m 2}=\frac{\left(1-\beta-\epsilon_{1}-\epsilon_{2}\right)\left(1+f_{b}\right)+\epsilon_{1}+\epsilon_{2} \tau_{r} \tau_{d} \tau_{c} /\left(\tau_{\lambda-b} \tau_{m 1} \tau_{t H}\right)}{\left(1-\beta-\epsilon_{1}-\epsilon_{2}\right)\left(1+f_{b}\right)+\epsilon_{1}+\epsilon_{2}}$

\section{Inter-stage Turbine Burner}

$$
\begin{aligned}
& \tau_{\lambda-i t b}=\frac{c_{p i t b} T_{t 4.5}}{c_{p c} T_{0}} \\
& \text { If (ITBoff) or }\left(\tau_{\lambda-i t b} \leq \tau_{\lambda-b} \tau_{t H} \tau_{m 1} \tau_{m 2}\right) \text { then } \\
& \qquad T_{t 4.5}=T_{t 4} \tau_{m 1} \tau_{t H} \tau_{m 2} \\
& f_{i t b}=0 \\
& \tau_{i t b}=1
\end{aligned}
$$


$\pi_{i t b}=1$

Goto 5

Endif

If $(\mathbf{C S H})$ then $f_{i t b}=\frac{\tau_{\lambda-i t b}-\tau_{\lambda-b} \tau_{t H} \tau_{m 1} \tau_{m 2}}{\eta_{i t b} h_{P R} /\left(c_{p c} T_{0}\right)-\tau_{\lambda-i t b}}$

Else

$T_{t 4.4}=T_{t 4} \tau_{t H} \tau_{m 1} \tau_{m 2}$

$\operatorname{ENTHALPY}\left(0, T_{t 4.5}, f_{b}, h_{t 4.4}, 0,0\right)$

Set initial values of fuel/air ratio at station 4.4 ,

$f_{4.5 i}=\frac{\tau_{\lambda-i t b}-\tau_{\lambda-b} \tau_{t H} \tau_{m 1} \tau_{m 2}}{\eta_{i t b} h_{P R} /\left(c_{p c} T_{0}\right)-\tau_{\lambda-i t b}}$

4

$\operatorname{ENTHALPY}\left(0, T_{t 4.5}, f_{4.5 i}, h_{t 4.5}, 0,0\right) f_{i t b}=\frac{h_{t 4.5}-h_{t 4.4}}{\eta_{i t b} h_{P R}-h_{t 4.5}}$

If $\left(\left|f_{b}+f_{i t b}-f_{4.5 i}\right|>0.0001\right)$, then $f_{4.5 i}=f_{b}+f_{i t b}$ and goto 4; Else

continue.

Endif

5

$\tau_{i t b}=\frac{T_{t 4.5}}{T_{0} \tau_{r} \tau_{c} \tau_{b} \tau_{t H} \tau_{m 1} \tau_{m 2}}$

$R_{i t b}=\frac{\gamma_{i t b}-1}{\gamma_{i t b}} c_{p i t b}$

\section{Low-Pressure Turbine}

$$
\begin{aligned}
\tau_{t L} & =1-\frac{\left[\left(\tau_{c L}-1\right)+\alpha\left(\tau_{f}-1\right)\right] \tau_{r}}{\left[\left(1-\beta-\epsilon_{1}-\epsilon_{2}\right)\left(1+f_{b}\right)+\epsilon_{1}+\epsilon_{2}\right]\left(1+f_{i t b}\right) \tau_{\lambda-i t b} \eta_{m L}} \\
\pi_{t L} & =\tau_{t L}^{\gamma_{i t b} /\left[\left(\gamma_{i t b}-1\right) e_{t L}\right]} \\
\eta_{t H} & =\frac{1-\tau_{t H}}{1-\tau_{t H}^{1 / l_{t H}}} \\
\eta_{t L} & =\frac{1-\tau_{\tau_{L}}}{1-\tau_{t L}^{1 / e_{t L}}}
\end{aligned}
$$

\section{Core Exhaust Stream}

\section{Convergent nozzle:}

$$
\begin{aligned}
& \frac{P_{t 9}}{P_{0}}=\pi_{r} \pi_{d} \pi_{c L} \pi_{c H} \pi_{b} \pi_{t H} \pi_{i t b} \pi_{t L} \pi_{n} \\
& \text { If }\left[\frac{P_{t 9}}{P_{0}} \geq\left(\frac{\gamma_{i t b}+1}{2}\right)^{\gamma_{i t b} /\left(\gamma_{i t b}-1\right)}\right] \text { then } \\
& M_{9}=1
\end{aligned}
$$




$$
\begin{aligned}
& \frac{P_{t 9}}{P_{9}}=\left(\frac{\gamma_{i t b}+1}{2}\right)^{\gamma_{i t b} /\left(\gamma_{i t b}-1\right)} \\
& \frac{P_{0}}{P_{9}}=\frac{P_{t 9} / P_{9}}{P_{t 9} / P_{0}}
\end{aligned}
$$

Else

$$
\begin{aligned}
& \frac{P_{0}}{P_{9}}=1 \\
& \frac{P_{t 9}}{P_{9}}=\frac{P_{t 9}}{P_{0}} \\
& M_{9}=\sqrt{\frac{2}{\gamma_{i t b}-1}\left[\left(\frac{P_{t 9}}{P_{9}}\right)^{\left(\gamma_{i t b}-1\right) / \gamma_{i t b}}-1\right]}
\end{aligned}
$$

Endif

\section{Convergent-Divergent nozzle:}

$$
\begin{aligned}
& \frac{P_{t 9}}{P_{9}}=\frac{P_{0}}{P_{9}} \pi_{r} \pi_{d} \pi_{c L} \pi_{c H} \pi_{b} \pi_{t H} \pi_{i t b} \pi_{t L} \pi_{n} \\
& M_{9}=\sqrt{\frac{2}{\gamma_{i t b}-1}\left[\left(\frac{P_{t 9}}{P_{9}}\right)^{\left(\gamma_{i t b}-1\right) / \gamma_{i t b}}-1\right]} \\
& \text { If }\left(M_{9} \geq 1\right) \text { then } M_{8}=1 ; \text { Else } M_{8}=M_{9} . \\
& \frac{T_{t 9}}{T_{0}}=\frac{T_{t 4.5} \tau_{t L}}{T_{0}} \\
& \frac{T_{9}}{T_{0}}=\frac{T_{t 9} / T_{0}}{\left(P_{t 9} / P_{9}\right)^{\left(\gamma_{i t b}-1\right) / \gamma_{i t b}}} \\
& \frac{V_{9}}{a_{0}}=M_{9} \sqrt{\frac{\gamma_{i t b} R_{i t b} T_{9}}{\gamma_{c} R_{c} T_{0}}}
\end{aligned}
$$

\section{Fan Bypass Stream}

\section{Convergent nozzle:}

$$
\begin{aligned}
& \frac{P_{t 19}}{P_{0}}=\tau_{r} \tau_{d} \tau_{f} \tau_{n f} \\
& \text { If }\left[\frac{P_{t 19}}{P_{0}} \geq\left(\frac{\gamma_{c}+1}{2}\right)^{\gamma_{c} /\left(\gamma_{c}-1\right)}\right] \text { then } \\
& M_{19}=1 \\
& \frac{P_{t 19}}{P_{19}}=\left(\frac{\gamma_{c}+1}{2}\right)^{\gamma_{c} /\left(\gamma_{c}-1\right)} \\
& \frac{P_{0}}{P_{19}}=\frac{P_{t 19} / P_{19}}{P_{t 19} / P_{0}}
\end{aligned}
$$

Else

$$
\begin{aligned}
& \frac{P_{0}}{P_{19}}=1 \\
& \frac{P_{t 19}}{P_{19}}=\frac{P_{t 19}}{P_{0}} \\
& M_{19}=\sqrt{\frac{2}{\gamma_{c}-1}\left[\left(\frac{P_{t 19}}{P_{19}}\right)^{\left(\gamma_{c}-1\right) / \gamma_{c}}-1\right]}
\end{aligned}
$$

Endif 


\section{Convergent-Divergent nozzle:}

$$
\begin{aligned}
& \frac{P_{t 19}}{P_{19}}=\frac{P_{0}}{P_{19}} \tau_{r} \tau_{d} \tau_{f} \tau_{n f} \\
& M_{19}=\sqrt{\frac{2}{\gamma_{c}-1}\left[\left(\frac{P_{t 19}}{P_{19}}\right)^{\left(\gamma_{c}-1\right) / \gamma_{c}}-1\right]} \\
& \text { If }\left(M_{19} \geq 1\right) \text { then } M_{18}=1 ; \text { Else } M_{18}=M_{19} . \\
& \frac{T_{t 19}}{T_{0}}=\tau_{f} \tau_{f} \\
& \frac{T_{19}}{T_{0}}=\frac{T_{t 19} / T_{0}}{\left(P_{t 19} / P_{19}\right)\left(\gamma_{c}-1\right) / \gamma_{c}} \\
& \frac{V_{19}}{a_{0}}=M_{19} \sqrt{\frac{T_{19}}{T_{0}}}
\end{aligned}
$$

\section{Aircraft Performance Parameters}

$$
\begin{aligned}
\frac{F_{c}}{\dot{m}_{c}}= & \frac{a_{0}}{g_{c}}\left\{\left[\left(1-\beta-\epsilon_{1}-\epsilon_{2}\right)\left(1+f_{b}\right)+\epsilon_{1}+\epsilon_{2}\right]\left(1+f_{i t b}\right) \frac{V_{9}}{a_{0}}-M_{0}+\right. \\
& {\left.\left[\left(1-\beta-\epsilon_{1}-\epsilon_{2}\right)\left(1+f_{b}\right)+\epsilon_{1}+\epsilon_{2}\right]\left(1+f_{i t b}\right) \frac{R_{i t b}}{R_{c}} \frac{T_{9} / T_{0}}{V_{9} / a_{0}} \frac{1}{\gamma_{c}}\left(1-\frac{P_{0}}{P_{9}}\right)\right\} }
\end{aligned}
$$$$
\frac{F_{f}}{\dot{m}_{f}}=\frac{a_{0}}{g_{c}}\left[\frac{V_{19}}{a_{0}}-M_{0}+\frac{T_{19} / T_{0}}{V_{19} / a_{0}} \frac{1}{\gamma_{c}}\left(1-\frac{P_{0}}{P_{19}}\right)\right]
$$$$
\frac{F}{\dot{m}_{0}}=\frac{\frac{F_{c}}{\dot{m}_{c}}+\alpha \frac{F_{f}}{\dot{m}_{f}}}{1+\alpha}
$$$$
S=\frac{\left(1-\beta-\epsilon_{1}-\epsilon_{2}\right) f_{b}+\left[\left(1-\beta-\epsilon_{1}-\epsilon_{2}\right)\left(1+f_{b}\right)+\epsilon_{1}+\epsilon_{2}\right] f_{i t b}}{\frac{F_{c}}{\dot{m}_{c}}+\alpha \frac{F_{f}}{\dot{m}_{f}}}
$$$$
\frac{\dot{W}_{\text {out }}}{\dot{m}_{c}}=\frac{1}{2 g_{c}}\left\{\left\{\left[\left(1-\beta-\epsilon_{1}-\epsilon_{2}\right)\left(1+f_{b}\right)+\epsilon_{1}+\epsilon_{2}\right] V_{9}^{2}-V_{0}^{2}\right\}+\alpha\left(V_{19}^{2}-V_{0}^{2}\right)\right\}
$$$$
\frac{\dot{Q}_{i n}}{\dot{m}_{c}}=\left(1-\beta-\epsilon_{1}-\epsilon_{2}\right) f_{b} h_{P R-b} \eta_{b}+\left[\left(1-\beta-\epsilon_{1}-\epsilon_{2}\right)\left(1+f_{b}\right)+\epsilon_{1}+\epsilon_{2}\right] f_{i t b} h_{P R-i t b} \eta_{i t b}
$$$$
\eta_{T H}=\frac{\dot{W}_{\text {out }} / \dot{m}_{c}}{\dot{Q}_{i} n / \dot{m}_{c}}
$$$$
\eta_{P}=\frac{F / \dot{m}_{0}(1+\alpha) V_{0}}{\dot{W}_{\text {out }} / \dot{m}_{c}}
$$

$\eta_{O}=\eta_{T H} \eta_{P}$ 


\section{Appendix E}

\section{Summary of Equations for Performance Cycle Analysis}

Appendix E summarizes the complete performance (or off-design) cycle analysis equations for the separate-exhaust, two-spool turbofan engine with an ITB and turbine cooling. The inputs, outputs and equations shown below follows the order of the solution in the computer code. This solution procedure for the off-design analysis are not as straight forward as that of the on-design analysis. It requires iterations for $\pi_{t H}$ and $\tau_{f}$ as depicted in Figure I.11.

\section{E.1 Inputs}

Performance choices:

Flight parameters: $\quad M_{0}, h, T_{0}, P_{0}$

Throttle settings: $\quad T_{t 4}, T_{t 4.5}$

Design constants:

$$
\begin{array}{ll}
\pi: & \pi_{d \max }, \pi_{b}, \pi_{i t b}, \pi_{n}, \pi_{n f} \\
\eta: & \eta_{t H}, \eta_{t L}, \eta_{c H}, \eta_{c L}, \eta_{f}, \eta_{m H}, \eta_{m L} \\
\text { Others: } & \beta, h_{P R} \\
& P_{0} / P_{9}, P_{0} / P_{19} \text { (for convergent-divergent nozzle only) }
\end{array}
$$

Reference Condition: 
Flight parameters: $\quad M_{0 R}, h_{R}, T_{0 R}, P_{0 R}$

Throttle settings: $\quad T_{t 4 R}, T_{t 4.5 R}$

Component behaviors: $\tau_{t H R}, \tau_{t L R}, \tau_{c H R}, \tau_{c L R}, \tau_{f R}$

$\pi_{t H R}, \pi_{t L R}, \pi_{c H R}, \pi_{c L R}, \pi_{f R}$

$M_{8 R}, M_{18 R}, f_{b R}, f_{i t b R}$

Others: $\quad F_{R}, S_{R}, \dot{m}_{0 R}, \eta_{t h R}, \eta_{P R}, \eta_{O R}, \alpha_{R}, \tau_{m 1 R}, \tau_{m 2 R}$

Engine control limits: $\quad T_{t 4 \max }, T_{t 4.5 \max }, \pi_{c H \max }, \pi_{f \max }$

$T_{t 3 \max }, P_{t 3 \max }, \% N_{L}, \% N_{H}$

\section{E.2 Outputs}

Overall performance: $F, S, \eta_{t h}, \eta_{P}, \eta_{O}, \dot{m}_{0}, \alpha$

Component behaviors: $\tau_{t H}, \tau_{t L}, \tau_{c H}, \tau_{c L}, \tau_{f}, \pi_{t H}, \pi_{t L}, \pi_{c H}, \pi_{c L}, \pi_{f}$

$f_{o}, f_{b}, f_{i t b}, \tau_{\lambda-b}, \tau_{\lambda-i t b}, M_{8}, M_{9}, M_{18}, M_{19}$

$\tau_{m 1}, \tau_{m 2}, A_{4.5} / A_{4.5 R}, A_{8} / A_{8 R}, A_{9} / A_{8}, A_{19} / A_{18}$

$M_{8}, M_{9}, M_{18}, M_{19}, \epsilon_{1}, \epsilon_{2}$

\section{E.3 Equations}

USATMOS76(alt,altR)

If (SI) then $T_{r e f}=288.6 \mathrm{~K}$; Else $T_{r e f}=516.67^{\circ} R$

$\operatorname{ENTHALPY}\left(1, T_{0}, 0,0, c_{p c}, \gamma_{c}\right) R_{c}=\frac{\gamma_{c}-1}{\gamma_{c}} c_{p c}$

\section{Reference Conditions}

$$
\begin{aligned}
& \tau_{r R}=1+\frac{\gamma_{c}-1}{2} M_{0 R}^{2} \\
& \pi_{r R}=\tau_{r R}^{\gamma_{c} /\left(\gamma_{c}-1\right)} \\
& \eta_{R \text { specR }}= \begin{cases}1 & \text { for } M_{0 R} \leq 1 \\
1-0.075\left(M_{0 R}-1\right)^{1.35} & \text { for } 1<M_{0 R}<5\end{cases}
\end{aligned}
$$


$\pi_{d R}=\pi_{d \max } \eta_{R \operatorname{spec} R}$

$\operatorname{MFP}\left(M_{18 R}\right)=M_{18 R} \sqrt{\frac{\gamma_{c} g_{c}}{R_{c}}}\left(1+\frac{\gamma_{c}-1}{2} M_{18 R}^{2}\right)^{\frac{\gamma_{c}+1}{2\left(1-\gamma_{c}\right)}}$

$\theta_{0 R}=\tau_{r R} \frac{T_{0 R}}{T_{r e f}}$

$\tau_{i t b R}=\frac{T_{t 4.5 R}}{T_{t 4 R} \tau_{t H R} \tau_{m 1 R} \tau_{m 2 R}}$

\section{Set initial values:}

$\dot{m}_{0}=\dot{m}_{0 R}, f_{b}=f_{b r}, f_{i t b}=f_{i t b R}, \pi_{t H}=\pi_{t H R}, \tau_{t H}=\tau_{t H R}$,

$\pi_{t L}=\pi_{t L R}, \tau_{t L}=\tau_{t L R}, \pi_{f}=\pi_{f R}, \tau_{f}=\tau_{f R}, \pi_{c H}=\pi_{c H R}, \tau_{c H}=\tau_{c H R}$,

$\pi_{c L}=\pi_{c L R}, \tau_{c L}=\tau_{c L R}, \tau_{m 1}=\tau_{m 1 R}, \tau_{m 2}=\tau_{m 2 R}$.

$\tau_{\lambda-b}=\frac{c_{p t} T_{t 4}}{c_{p c} T_{0}}$

\section{Preliminary Computations}

$$
\begin{aligned}
& \tau_{r}=1+\frac{\gamma_{c}-1}{2} M_{0}^{2} \\
& \pi_{r}=\tau_{r}^{\gamma_{c} /\left(\gamma_{c}-1\right)} \\
& \eta_{R s p e c}= \begin{cases}1 & \text { for } M_{0} \leq 1 \\
1-0.075\left(M_{0}-1\right)^{1.35} & \text { for } 1<M_{0}<5\end{cases} \\
& \pi_{d}=\pi_{d \text { max }} \eta_{\text {Rspec }} \\
& a_{0}=\sqrt{\gamma_{c} R_{c} g_{c} T_{0}} \\
& M F P\left(M_{0}\right)=M_{0} \sqrt{\frac{\gamma_{c} g_{c}}{R_{c}}}\left(1+\frac{\gamma_{c}-1}{2} M_{0}^{2}\right)^{\frac{\gamma_{c}+1}{2\left(1-\gamma_{c}\right)}} \\
& T_{t 0}=T_{0} \tau_{r} \\
& P_{t 0}=P_{0} \tau_{r}^{\gamma_{c} /\left(\gamma_{c}-1\right)}
\end{aligned}
$$

\section{Control Limits}

$\theta_{0}=\tau_{r} \frac{T_{0}}{T_{r e f}}$

If $\left(\theta_{0} \geq \theta_{0 R}\right)$ then $T_{t 4}=T_{t 4 \max }$; Else $T_{t 4}=T_{t 4 \max }\left(\frac{\theta_{0}}{\theta_{0 R}}\right)$.

If (ITBon) then

$$
\text { If }\left(\theta_{0} \geq \theta_{0 R}\right) \text { then } T_{t 4.5}=T_{t 4.5 \max } ; \text { Else } T_{t 4.5}=T_{t 4.5 \max }\left(\frac{\theta_{0}}{\theta_{0 R}}\right) \text {. }
$$

Endif

12

$\operatorname{ENTHALPY}\left(1, T_{t}, f_{b}, 0, c_{p t}, \gamma_{t}\right)$ 
$R_{t}=\frac{\gamma_{t}-1}{\gamma_{t}} c_{p t}$

1

$\operatorname{ENTHALPY}\left(1, T_{t 4.5}, f_{i t b}, 0, c_{p i t b}, \gamma_{i t b}\right)$

$R_{i t b}=\frac{\gamma_{i t b}-1}{\gamma_{i t b} c_{p i t b}}$

$\tau_{\lambda-i t b}=\frac{c_{p i t b} T_{t 4.5}}{c_{p c} T_{0}}$

If $\left(\tau_{\lambda-i t b} \leq \tau_{\lambda-b} \tau_{t H}\right)$ then REINITIAL(1).

If (ITBon) then

$$
\tau_{i t b}=\frac{T_{t 4.5}}{T_{t 4} \tau_{t H} \tau_{m 1} \tau_{m 2}}
$$

Else

$$
\begin{aligned}
& \tau_{i t b}=1 \\
& \tau_{i t b R}=1 \\
& \tau_{\lambda-i t b}=\frac{c_{p t} T_{t 4}}{c_{p c} T_{0}} \tau_{t H} \\
& T_{t 4.5}=T_{t 4} \tau_{t H}
\end{aligned}
$$

$\operatorname{ENTHALPY}\left(1, T_{t 4.5}, 0,0, c_{p i t b}, \gamma_{i t b}\right)$

$$
R_{i t b}=\frac{\gamma_{i t b}-1}{\gamma_{i t b} c_{p i t b}}
$$

Endif

$$
\begin{aligned}
& \frac{A_{4.5}}{A_{4.5 R}}=\left(\frac{\tau_{i t b}}{\tau_{i t b R}}\right)^{n} \\
& \frac{A_{8}}{A_{8 R}}=\left(\frac{\tau_{i t b}}{\tau_{i t b R}}\right)^{m}
\end{aligned}
$$

\section{High-Pressure Turbine}

$\operatorname{COOLING}\left(T_{t 4}, \tau_{c}, \tau_{\lambda-b}, \beta, \epsilon_{1}, \epsilon_{2}\right)$

$\tau_{m 1}=\frac{\left(1-\beta-\epsilon_{1}-\epsilon_{2}\right)\left(1+f_{b}\right)+\epsilon_{1} \tau_{r} \tau_{d} \tau_{c} / \tau_{\lambda-b}}{\left(1-\beta-\epsilon_{1}-\epsilon_{2}\right)\left(1+f_{b}\right)+\epsilon_{1}}$

$\tau_{t H}=1-\eta_{t H}\left\{1-\pi_{t H}^{\left(\gamma_{t}-1\right) / \gamma_{t}}\right\}$

$\pi_{t H N}=\frac{\sqrt{\tau_{t H} \tau_{i t b} \tau_{m 1} \tau_{m 2}}}{\sqrt{\tau_{t H R} \tau_{i t b R} \tau_{m 1 R} \tau_{m 2 R}}} \frac{A_{4.5 R}}{A_{4.5}} \pi_{t H R}$

Is $\left|\pi_{t H N}-\pi_{t H}\right| \leq 0.00001$ ? If so, then continue. If not, set $\pi_{t H}=\pi_{t H N}$ and return to 1 .

$\tau_{m 2}=\frac{\left(1-\beta-\epsilon_{1}-\epsilon_{2}\right)\left(1+f_{b}\right)+\epsilon_{1}+\epsilon_{2} \tau_{r} \tau_{d} \tau_{c} /\left(\tau_{\lambda-b} \tau_{m 1} \tau_{m 2}\right)}{\left(1-\beta-\epsilon_{1}-\epsilon_{2}\right)\left(1+f_{b}\right)+\epsilon_{1}+\epsilon_{2}}$ 


\section{High-Pressure Compressor}

2

$\tau_{c H}=1+\eta_{m H}\left[\left(1-\beta-\epsilon_{1}-\epsilon_{2}\right)\left(1+f_{b}\right)+\epsilon_{1}\right] \frac{\tau_{\lambda-b}\left(1-\tau_{t H}\right) \tau_{m 1}}{\tau_{r} \tau_{c L}}$

$\pi_{c H}=\left\{1+\eta_{c H}\left(\tau_{c H}-1\right)\right\}^{\gamma_{c} /\left(\gamma_{c}-1\right)}$

If $\left[\operatorname{Abs}\left(\pi_{c H}-\pi_{c H \text { max }}>0.001\right)\right]$ then $\operatorname{ENGCONTROL}\left(\pi_{c} H, T_{t 4}\right)$ and goto 12.

\section{Main Burner}

If $(\mathbf{C S H})$ then

$$
f_{b}=\frac{\tau_{\lambda-b}-\tau_{r} \tau_{c L} \tau_{c H}}{\eta_{b} h_{P R} / h_{0}-\tau_{\lambda-b}}
$$

Else

$$
T_{t 3}=T_{0} \tau_{r} \tau_{c L} \tau_{c H}
$$

$\operatorname{ENTHALPY}\left(0, T_{t 3}, 0, h_{t 3}, 0,0\right)$

Set initial value of fuel/air ratio at station $4, f_{4 i}=\frac{\tau_{\lambda-b}-\tau_{r} \tau_{c L} \tau_{c} H}{\eta_{b} h_{P R} / h_{0}-\tau_{\lambda-b}}$

3

ENTHALPY $\left(0, T_{t 4}, f_{4 i}, h_{t} 4,0,0\right)$

$f_{b}=\frac{h_{t 4}-h_{t 3}}{\eta_{b} h_{P R}-h_{t 4}}$

If $\left(\left|f_{b}-f_{4 i}\right|>0.0001\right)$, then $f_{4 i}=f_{b}$ and goto 3 ; Else continue.

Endif

11

\section{Interstage Turbine Burner}

If (ITBoff) then $f_{i t b}=0, \gamma_{i t b}=\gamma_{t}, c_{p i t b}=c_{p t}, R_{i t b}=R_{t}$, and goto 5 .

If $(\mathbf{C S H})$ then $\quad f_{i t b}=\frac{\tau_{\lambda-i t b}-\tau_{\lambda-b} \tau_{t H} \tau_{m 1} \tau_{m 2}}{\eta_{i t b} h_{P R} / h_{0}-\tau_{\lambda-i t b}}$

Else

$T_{t 4.4}=T_{t 4} \tau_{t H} \tau_{m 1} \tau_{m 2}$

$\operatorname{ENTHALPY}\left(0, T_{t 4.5}, f_{b}, h_{t 4.4}, 0,0\right)$

Set initial value of fuel/air ratio at station $4.4, f_{4.5 i}=\frac{\tau_{\lambda-i t b}-\tau_{\lambda-b} \tau_{t H} \tau_{m 1} \tau_{m 2}}{\eta_{i t b} h_{P R} / h_{0}-\tau_{\lambda-i t b}}$ 4

$\operatorname{ENTHALPY}\left(0, T_{t 4.5}, f_{4.5 i}, h_{t 4.5}, 0,0\right)$ 


$$
\begin{aligned}
& f_{i t b}=\frac{h_{t 4.5}-h_{t 4.4}}{\eta_{i t b} h_{P R}-h_{t 4.5}} \\
& \text { If }\left(\left|f_{b}+f_{i t b}-f_{4.5 i}\right|>0.0001\right) \text {, then } f_{4.5 i}=f_{b}+f_{i t b} \text { and goto 4; Else }
\end{aligned}
$$

continue.

EndIf

$M F P\left(M_{8 R}\right)=M_{8 R} \sqrt{\frac{\gamma_{i t b} g_{c}}{R_{i t b}}}\left(1+\frac{\gamma_{i t b}-1}{2} M_{8 R}^{2}\right)^{\frac{\gamma_{i t b}+1}{2\left(1-\gamma_{i t b}\right)}}$

5

Fan and Low-Pressure Compressor Pressure Ratios

$$
\begin{aligned}
& \pi_{f}=\left\{1+\eta_{f}\left(\tau_{f}-1\right)\right\}^{\gamma_{c} /\left(\gamma_{c}-1\right)} \\
& \pi_{c L}=\left\{1+\eta_{c L}\left(\tau_{c L}-1\right)\right\}^{\gamma_{c} /\left(\gamma_{c}-1\right)}
\end{aligned}
$$

\section{Core Exhaust Stream}

For convergent nozzle:

$$
\begin{gathered}
\frac{P_{t 9}}{P_{0}}=\pi_{r} \pi_{d} \pi_{c L} \pi_{c H} \pi_{b} \pi_{t H} \pi_{i t b} \pi_{t L} \pi_{n} \\
\text { If }\left[\frac{P_{t 9}}{P_{0}} \geq\left(\frac{\gamma_{i t b}+1}{2}\right)^{\gamma_{i t b} /\left(\gamma_{i t b}-1\right)}\right] \text { then } \\
M_{9}=1 \\
\frac{P_{t 9}}{P_{9}}=\left(\frac{\gamma_{i t b}+1}{2}\right)^{\gamma_{i t b} /\left(\gamma_{i t b}-1\right)} \\
\frac{P_{0}}{P_{9}}=\frac{P_{t 9} / P_{9}}{P_{t 9} / P_{0}}
\end{gathered}
$$

Else

$$
\begin{aligned}
& \frac{P_{0}}{P_{9}}=1 \\
& \frac{P_{t 9}}{P_{9}}=\frac{P_{t 9}}{P_{0}} \\
& M_{9}=\sqrt{\frac{2}{\gamma_{i t b}-1}\left[\left(\frac{P_{t 9}}{P_{9}}\right)^{\left(\gamma_{i t b}-1\right) / \gamma_{i t b}}-1\right]}
\end{aligned}
$$

Endif

For convergent-divergent nozzle:

$$
\begin{aligned}
& \frac{P_{t 9}}{P_{9}}=\frac{P_{0}}{P_{9}} \pi_{r} \pi_{d} \pi_{c L} \pi_{c H} \pi_{b} \pi_{t H} \pi_{i t b} \pi_{t L} \pi_{n} \\
& M_{9}=\sqrt{\frac{2}{\gamma_{i t b}-1}\left[\left(\frac{P_{t 9}}{P_{9}}\right)^{\left(\gamma_{i t b}-1\right) / \gamma_{i t b}}-1\right]} \\
& \text { If }\left(M_{9} \geq 1\right) \text { then } M_{8}=1 \text { Else } M_{8}=M_{9} .
\end{aligned}
$$




\section{For both:}

$$
\begin{aligned}
& \operatorname{MFP}\left(M_{8}\right)=M_{8} \sqrt{\frac{\gamma_{i t b} g_{c}}{R_{i t b}}}\left(1+\frac{\gamma_{i t b}-1}{2} M_{8}^{2}\right)^{\frac{\gamma_{i t b}+1}{2\left(1-\gamma_{i t b}\right)}} \\
& \operatorname{MFP}\left(M_{9}\right)=M_{9} \sqrt{\frac{\gamma_{i t b} g_{c}}{R_{i t b}}}\left(1+\frac{\gamma_{i t b}-1}{2} M_{9}^{2}\right)^{\frac{\gamma_{i t b}+1}{2\left(1-\gamma_{i t b}\right)}} \\
& \frac{A_{9}}{A_{8}}=\frac{M F P\left(M_{8}\right)}{\pi_{n} M F P\left(M_{9}\right)}
\end{aligned}
$$

\section{Fan Bypass Stream}

For convergent nozzle:

$$
\begin{aligned}
& \frac{P_{t 19}}{P_{0}}=\tau_{r} \tau_{d} \tau_{f} \tau_{n f} \\
& \text { if }\left[\frac{P_{t 19}}{P_{0}} \geq\left(\frac{\gamma_{c}+1}{2}\right)^{\gamma_{c} /\left(\gamma_{c}-1\right)}\right] \text { then } \\
& \qquad \begin{aligned}
M_{19} & =1 \\
\frac{P_{t 19}}{P_{19}} & =\left(\frac{\gamma_{c}+1}{2}\right)^{\gamma_{c} /\left(\gamma_{c}-1\right)} \\
\frac{P_{0}}{P_{19}} & =\frac{P_{t 19} / P_{19}}{P_{t 19} / P_{0}}
\end{aligned}
\end{aligned}
$$

Else

$$
\begin{aligned}
& \frac{P_{0}}{P_{19}}=1 \\
& \frac{P_{t 19}}{P_{19}}=\frac{P_{t 19}}{P_{0}} \\
& M_{19}=\sqrt{\frac{2}{\gamma_{c}-1}\left[\left(\frac{P_{t 19}}{P_{19}}\right)^{\left(\gamma_{c}-1\right) / \gamma_{c}}-1\right]}
\end{aligned}
$$

Endif

\section{For convergent-divergent nozzle:}

$$
\begin{aligned}
& \frac{P_{t 19}}{P_{19}}=\frac{P_{0}}{P_{19}} \tau_{r} \tau_{d} \tau_{f} \tau_{n f} \\
& M_{19}=\sqrt{\frac{2}{\gamma_{c}-1}\left[\left(\frac{P_{t 19}}{P_{19}}\right)^{\left(\gamma_{c}-1\right) / \gamma_{c}}-1\right]}
\end{aligned}
$$

For both:

$$
\begin{aligned}
& \operatorname{MFP}\left(M_{18}\right)=M_{18} \sqrt{\frac{\gamma_{c} g_{c}}{R_{c}}}\left(1+\frac{\gamma_{c}-1}{2} M_{18}^{2}\right)^{\frac{\gamma_{c}+1}{2\left(1-\gamma_{c}\right)}} \\
& \operatorname{MFP}\left(M_{19}\right)=M_{19} \sqrt{\frac{\gamma_{c} g_{c}}{R_{c}}}\left(1+\frac{\gamma_{c}-1}{2} M_{19}^{2}\right)^{\frac{\gamma_{c}+1}{2\left(1-\gamma_{c}\right)}} \\
& \frac{A_{19}}{A_{18}}=\frac{M F P\left(M_{18}\right)}{\pi_{n f} M F P\left(M_{19}\right)}
\end{aligned}
$$




\section{Fan Bypass Ratio}

$\alpha=\alpha_{R} \frac{\pi_{c L R} \pi_{c H R} / \pi_{f R}}{\pi_{c L} \pi_{c H} / \pi_{f}} \sqrt{\frac{T_{t 4} / T_{t 4 R}}{\tau_{r} \tau_{f} /\left(\tau_{r R} \tau_{f R}\right)}} \frac{M F P\left(M_{18}\right)}{M F P\left(M_{18 R}\right)}$

\section{Fan and Low-Pressure Compressor Temperature Ratios}

$$
\begin{aligned}
& \tau_{f N}=1+\left(\tau_{f R}-1\right) \eta_{m L} \frac{\tau_{\lambda-i t b}}{\tau_{r}}\left\{\frac{\left(1-\tau_{t L}\right)\left[\left(1-\beta-\epsilon_{1}-\epsilon_{2}\right)\left(1+f_{b}\right)+\epsilon_{1}+\epsilon_{2}\right]\left(1+f_{i t b}\right)}{\tau_{c L R}-1+\alpha\left(\tau_{f R}-1\right)}\right\} \\
& \tau_{c L}=1+\left(\tau_{f N}-1\right) \frac{\tau_{c L R}-1}{\tau_{f R}-1}
\end{aligned}
$$

\section{Low-Pressure Turbine}

$\tau_{t L}=1-\eta_{t L}\left\{1-\pi_{t L}^{\left(\gamma_{i t b}-1\right) / \gamma_{i t b}}\right\}$

$\pi_{t L}=\pi_{t L R} \sqrt{\frac{\tau_{t L}}{\tau_{t L R}}} \frac{A_{8 R}}{A_{8}} \frac{A_{4.5}}{A_{4.5 R}} \frac{M F P\left(M_{8 R}\right)}{M F P\left(M_{8}\right)}$

Is $\left|\tau_{f N}-\tau_{f}\right| \leq 0.0001$ ? If so, then continue. If not, set $\tau_{f}=\tau_{f N}$ and return to 2 .

\section{Engine Mass Flow $\left(\dot{m}_{0}\right)$}

$\dot{m}_{0}=\dot{m}_{0 R} \frac{1+\alpha}{1+\alpha_{R}} \frac{P_{0} \pi_{r} \pi_{d} \pi_{c L} \pi_{c H}}{P_{0 R} \pi_{r R} \pi_{d R} \pi_{c L R} \pi_{c H R}} \sqrt{\frac{T_{t 4 R}}{T_{t 4}}}$

\section{Engine Controls}

If (ITBon) and $\left(\pi_{f \max }>0\right)$ then $\operatorname{ENGCONTROL}\left(\pi_{f}, T_{t 4.5}\right)$ and goto 11.

If (ITBoff) and $\left(\pi_{\text {fmax }}>0\right)$ then $\operatorname{ENGCONTROL}\left(\pi_{f}, T_{t 4}\right)$ and goto 12.

If $\left(\pi_{c H \text { max }}>0\right)$ then ENGCONTROL $\left(\pi_{c H}, T_{t 4}\right)$ and goto 12 .

If $\left(P_{t 3 \max }>0\right)$ then ENGCONTROL $\left(P_{t 3}, T_{t 4}\right)$ and goto 12 .

If $\left(T_{t 3 \max }>0\right)$ then ENGCONTROL $\left(T_{t 3}, T_{t 4}\right)$ and goto 12 .

If (ITBon) and ( $\left.\% N_{L \max }>0\right)$ then ENGCONTROL $\left(\% N_{L}, T_{t 4.5}\right)$ and goto 11.

If (ITBoff) and ( $\left.\% N_{L \max }>0\right)$ then ENGCONTROL $\left(\% N_{L}, T_{t 4}\right)$ and goto 12 .

If $\left(\% N_{H \max }>0\right)$ then ENGCONTROL $\left(\% N_{H}, T_{t 4}\right)$ and goto 12.

[Note: Please refer to Section F.5.4 for more description in

$\left.\operatorname{ENGCONTROL}\left(Q, T_{t}\right) \cdot\right]$ 


\section{Overall Engine Performance}

$$
\begin{aligned}
& \frac{T_{9}}{T_{0}}=\frac{T_{t 4.5} \tau_{t L} / T_{0}}{\left(P_{t 9} / P_{9}\right)^{\left(\gamma_{i t b}-1\right) / \gamma_{i t b}}} \\
& \frac{T_{19}}{T_{0}}=\frac{\tau_{r} \tau_{f}}{\left(P_{t 19} / P_{19}\right)^{\left(\gamma_{c}-1\right) / \gamma_{c}}} \\
& \frac{T_{t 19}}{T_{0}}=\tau_{f} \tau_{f} \\
& a_{9}=\sqrt{\gamma_{i t b} R_{i t b} g_{c} T_{9}} \\
& a_{19}=\sqrt{\gamma_{c} R_{c} g_{c} T_{19}} \\
& V_{9}=M_{9} a_{9} \\
& V_{19}=M_{19} a_{19} \\
& f_{b, o v r}=\left(1-\beta-\epsilon_{1}-\epsilon_{2}\right) f_{b} \\
& f_{i t b, o v r}=\left[\left(1-\beta-\epsilon_{1}-\epsilon_{2}\right)\left(1+f_{b}\right)+\epsilon_{1}+\epsilon_{2}\right] f_{i t b} \\
& f_{0}=f_{b, o v r}+f_{i t b, o v r} \\
& \frac{F_{c}}{\dot{m}_{c}}=\frac{a_{0}}{g_{c}}\left\{\left[\left(1-\beta-\epsilon_{1}-\epsilon_{2}\right)\left(1+f_{b}\right)+\epsilon_{1}+\epsilon_{2}\right]\left(1+f_{i t b}\right) \frac{V_{9}}{a_{0}}-M_{0}+\right. \\
& \left.\left[\left(1-\beta-\epsilon_{1}-\epsilon_{2}\right)\left(1+f_{b}\right)+\epsilon_{1}+\epsilon_{2}\right]\left(1+f_{i t b}\right) \frac{R_{i t b}}{R_{c}} \frac{T_{9} / T_{0}}{V_{9} / a_{0}} \frac{1}{\gamma_{c}}\left(1-\frac{P_{0}}{P_{9}}\right)\right\} \\
& \frac{F_{f}}{\dot{m}_{f}}=\frac{a_{0}}{g_{c}}\left[\frac{V_{19}}{a_{0}}-M_{0}+\frac{T_{19} / T_{0}}{V_{19} / a_{0}} \frac{1}{\gamma_{c}}\left(1-\frac{P_{0}}{P_{19}}\right)\right] \\
& \frac{F}{\dot{m}_{0}}=\frac{\frac{F_{c}}{\dot{m}_{c}}+\alpha \frac{F_{f}}{\dot{m}_{f}}}{1+\alpha} \\
& F=\dot{m}_{0}\left(\frac{F}{\dot{m}_{0}}\right) \\
& S=\frac{f_{0}}{F / \dot{m}_{0}(1+\alpha)} \\
& \frac{\dot{W}_{\text {out }}}{\dot{m}_{c}}=\frac{1}{2 g_{c}}\left\{\left\{\left[\left(1-\beta-\epsilon_{1}-\epsilon_{2}\right)\left(1+f_{b}\right)+\epsilon_{1}+\epsilon_{2}\right] V_{9}^{2}-V_{0}^{2}\right\}+\alpha\left(V_{19}^{2}-V_{0}^{2}\right)\right\} \\
& \frac{\dot{Q}_{i n}}{\dot{m}_{c}}=\left(1-\beta-\epsilon_{1}-\epsilon_{2}\right) f_{b} h_{P R-b} \eta_{b}+\left[\left(1-\beta-\epsilon_{1}-\epsilon_{2}\right)\left(1+f_{b}\right)+\epsilon_{1}+\epsilon_{2}\right] f_{i t b} h_{P R-i t b} \eta_{i t b} \\
& \eta_{P}=\frac{F / \dot{m}_{0}(1+\alpha) V_{0}}{\dot{W}_{\text {out }} / \dot{m}_{c}} \\
& \eta_{T H}=\frac{\dot{W}_{\text {out }} / \dot{m}_{c}}{\dot{Q}_{i} n / \dot{m}_{c}} \\
& \eta_{O}=\eta_{T H} \eta_{P} \\
& A_{0}=\frac{\dot{m}_{0} \sqrt{T_{t 0}}}{P_{t 0} M F P\left(M_{0}\right)} \\
& \% N_{L}=100 \sqrt{\frac{T_{0} \tau_{r}\left(\tau_{f}-1\right)}{T_{0 R} \tau_{r R}\left(\tau_{f R}-1\right)}}
\end{aligned}
$$


$\% N_{H}=100 \sqrt{\frac{T_{0} \tau_{r} \tau_{c L}\left(\tau_{c H}-1\right)}{T_{0 R} \tau_{r R} \tau_{c L R}\left(\tau_{c H R}-1\right)}}$ 


\section{Appendix F}

\section{Engine Control Limit System}

\section{F.1 Introduction}

Normally, the designers of an aircraft gas turbine engine need to consider the engine control system so that the newly-designed or improved gas turbine engine operates well within its flight envelope. For example, the throttle setting needs to be controlled so that the burner exit temperature will never exceed the turbine blade maximum temperature. Another example is that fan or compressor surges or stalls should not occur during the operation.

Therefore, engine's control system plays a significant role in this engine performance cycle analysis because of the several advantages: (1) maintain stable airflow, internal pressures and temperatures, and rotor speeds within safe operating limits; (2) avoid significant speed, pressure or temperature variation, and stalls or surges [34]. In addition, it also demonstrates how an aircraft engine behaves away from their reference or on-design conditions.

The engine's control system in this study is specifically designed for a separate-flow, dual-spool, uncooled turbofan engine with ITB. Moreover, the development of the equations is based on the foundational materials found in [33] and [34]. 


\section{F.2 Variables of Engine Control System}

\section{F.2.1 Dimensionaless Freestream Total Temperature}

Due to its significant influence on overall behavior of the turbofan engine, a dimensionless form of the freestream total temperature is defined, i.e. the ratio of the freestream total temperature to the sea-level static $(S L S)$ temperature of the standard atmosphere, as shown below [34]:

$$
\theta_{0}=\frac{T_{t 0}}{T_{\text {ref }}}=\frac{T_{0}\left\{1+\frac{\gamma_{c}-1}{2} M_{0}^{2}\right\}}{T_{\text {ref }}}=\tau_{r} \frac{T_{0}}{T_{\text {ref }}}
$$

(Note: $\left.T_{r e f}=T_{s t d}\right)$

This property is called theta $0\left(\theta_{0}\right)$ and is used to combine two parameters, i.e., altitude $\left(T_{0}\right)$ and flight condition $\left(M_{0}\right)$ into a single parameter. Therefore, every point in the flight envelope has a specific value of $\theta_{0}$.

In addition, $\theta_{0}$ has the following properties:

1. $\theta_{0}=1$ at sea level static conditions;

2. $\theta_{0}$ can be greater or less than 1.0 ;

3. $\theta_{0}$ depends only on Mach number between $36.5 \mathrm{kft}$ and $65 \mathrm{kft}(11.12 \mathrm{~km}$ and $19.81 \mathrm{~km})$.

For a reference-point engine, another property, i.e., theta $0 R\left(\theta_{0 R}\right)$ is defined and can be determined in a similar way:

$$
\theta_{0 R}=\frac{T_{t 0 R}}{T_{r e f}}=\frac{T_{0 R}\left\{1+\frac{\gamma_{c}-1}{2} M_{0 R}^{2}\right\}}{T_{r e f}}=\tau_{r R} \frac{T_{0 R}}{T_{r e f}}
$$




\section{F.2.2 Theta Break}

Normally, the change in shape of the performance curve occurs at simultaneous maximum of $\pi_{c}$ and $T_{t 4}$. In Figure F.1, it is clear that the control logic must switch from limiting $\pi_{c}$ to limiting $T_{t 4}$ at this point of maximum $\pi_{c}$ and $T_{t 4}$. This unique point is known as the theta break $\left(\theta_{0 b r e a k}\right)$.

\section{F.3 Types of Control Limits}

In this study, only three types of engine control limits will be considered, i.e., turbine blade maximum temperature, compressor limitations, and engine speeds.

\section{F.3.1 Material Limitation}

It is known that an increase in permissible turbine inlet temperature will lead to a reduced specific fuel consumption and increased specific thrust. Although the current advanced technology in material allows higher inlet turbine temperature, it is still essential to limit $T_{t 4}$ or $T_{4.5}$ for safety purpose.

\section{F.3.2 Compressor Pressure Ratio}

Three limits, namely, $\pi_{c \max }, P_{t 3 \max }$ and $T_{t 3 \max }$, are selected to stabilize the internal behavior of the compressors and avoid compressor surges or stalls.

\section{F.3.3 Engine Speed}

As shown in section 5.2.7 of [34, the change in total enthalpy across a compressor or a fan is proportional to the rotational speed $N$ squared for a calorically perfert gas.

\section{Low-Pressure Spool}

For a LPC, it can be expressed as follow: 


$$
h_{t 2.5}-h_{t 2}=K_{1} N_{L P}^{2}
$$

Using the referencing technique, equation (F.3) it can be rewritten as:

$$
\begin{aligned}
\frac{N_{L P}}{N_{L P R}} & =\sqrt{\frac{h_{t 2.5}-h_{t 2}}{h_{t 2.5 R}-h_{t 2 R}}}=\sqrt{\frac{h_{t 2}\left(\tau_{c L}-1\right)}{h_{t 2 R}\left(\tau_{c L R}-1\right)}} \\
& =\sqrt{\frac{h_{t 0}\left(\tau_{c L}-1\right)}{h_{t 0 R}\left(\tau_{c L R}-1\right)}} \cong \sqrt{\frac{T_{t 0}\left(\tau_{c L}-1\right)}{T_{t 0 R}\left(\tau_{c L R}-1\right)}} \\
& =\sqrt{\frac{\theta_{0}\left(\tau_{c L}-1\right)}{\theta_{0 R}\left(\tau_{c L R}-1\right)}}
\end{aligned}
$$

Since the LPC and the fan are on the same shaft, the enthalpy rise across the LPC will be proportional to the enthalpy rise across the fan during normal operation [34].

$$
\frac{\tau_{c L}-1}{\tau_{f}-1}=\frac{\tau_{c L R}-1}{\tau_{f R}-1}
$$

Then, equation (F.4) can be rewritten as

$$
\frac{N_{L P}}{N_{L P R}}=\sqrt{\frac{\theta_{0}\left(\tau_{f}-1\right)}{\theta_{0 R}\left(\tau_{f R}-1\right)}}
$$

\section{High-Pressure Spool}

For a HPC, it is in the similar form:

$$
h_{t 3}-h_{t 2.5}=K_{1} N_{H P}^{2}
$$

Using the referencing technique, eqn. (F.7) can be rewritten as: 


$$
\begin{aligned}
\frac{N_{H P}}{N_{H P R}} & =\sqrt{\frac{h_{t 3}-h_{t 2.5}}{h_{t 3 R}-h_{t 2.5 R}}}=\sqrt{\frac{h_{t 2.5}\left(\tau_{c H}-1\right)}{h_{t 2.5 R}\left(\tau_{c H R}-1\right)}} \\
& =\sqrt{\frac{h_{t 0} \tau_{c L}\left(\tau_{c H}-1\right)}{h_{t 0 R} \tau_{c L R}\left(\tau_{c H R}-1\right)}} \cong \sqrt{\frac{T_{t 0} \tau_{c L}\left(\tau_{c H}-1\right)}{T_{t 0 R} \tau_{c L R}\left(\tau_{c H R}-1\right)}} \\
& =\sqrt{\frac{\theta_{0} \tau_{c L}\left(\tau_{c H}-1\right)}{\theta_{0 R} \tau_{c L R}\left(\tau_{c L R}-1\right)}}
\end{aligned}
$$

\section{F.4 Relationship between $T_{t 4}, T_{t 4.5}$ and $\pi_{c}$}

\section{F.4.1 High-Pressure Compressor}

From a power balance between HPT and HPC, one will obtain the performance equation for the total temperature ratio of the $\mathrm{HPC}$ as:

$$
\tau_{c H}=1+\eta_{m H}\left[\left(1-\beta-\epsilon_{1}-\epsilon_{2}\right)\left(1+f_{b}\right)+\epsilon_{1}\right] \frac{\tau_{\lambda-b}\left(1-\tau_{t H}\right)}{\tau_{r} \tau_{c L}}
$$

Using HPC efficiency, the HPC total pressure is given by:

$$
\pi_{c H}=\left\{1+\eta_{c H}\left(\tau_{c H}-1\right)\right\}^{\gamma_{c} /\left(\gamma_{c}-1\right)}
$$

From equation (7) in [28],

$$
\tau_{\lambda-b}=\frac{c_{p t} T_{t 4}}{c_{p c} T_{0}}
$$

Rearranging equation (F.1), one obtains

$$
\tau_{r}=\theta_{0} \frac{T_{r e f}}{T_{0}}
$$

Substitute these two equations into (F.9) yields

$$
\tau_{c H}=1+\eta_{m H}\left[\left(1-\beta-\epsilon_{1}-\epsilon_{2}\right)\left(1+f_{b}\right)+\epsilon_{1}\right]\left(1-\tau_{t H}\right) \frac{c_{p t} T_{t 4}}{c_{p c} T_{0}}\left(\frac{T_{0}}{T_{r e f} \theta_{0}}\right) \frac{1}{\tau_{c L}}
$$


It is assumed that choking occurs at station 4. Consequently, for a cooled turbine with constant specific heats in this study, the value of $\tau_{t H}$ will remain constant. (pp.144 of [34]). In addition, $f_{b}$ is negligible when compared to unity. Then, let

$$
C_{0}=\eta_{m H}\left[\left(1-\beta-\epsilon_{1}-\epsilon_{2}\right)\left(1+f_{b}\right)+\epsilon_{1}\right]\left(1-\tau_{t H}\right)\left(\frac{c_{p t}}{c_{p c}}\right)\left(\frac{1}{T_{r e f}}\right)
$$

and eq.(F.13) becomes

$$
\tau_{c H}=1+C_{0} \frac{T_{t 4}}{\theta_{0}} \frac{1}{\tau_{c L}}
$$

Substituting F.15) into $\mathrm{F.10}$ gives

$$
\pi_{c H}=\left\{1+C_{1} \frac{T_{t 4}}{\theta_{0}} \frac{1}{\tau_{c L}}\right\}^{\gamma_{c} /\left(\gamma_{c}-1\right)}
$$

where

$$
C_{1}=C_{0} \eta_{c H}
$$

Compared to equation (D.3) of [34] (pp.525), $\pi_{c H}$ is not only a function of throttle setting $\left(T_{t 4}\right)$ and flight conditions $\left(\theta_{0}\right)$, but also a function of $\tau_{c L}$ :

$$
\pi_{c H}=f\left(\frac{T_{t 4}}{\theta_{0}}, \tau_{c L}\right)
$$

\section{F.4.2 Low-Pressure Compressor}

From a power balance between LPC, fan and LPT, one will obtain the performance equation for the total temperature ratio of the LPT:

$\alpha\left(\tau_{f}-1\right)+\left(\tau_{c L}-1\right)=\eta_{m L}\left[\left(1-\beta-\epsilon_{1}-\epsilon_{2}\right)\left(1+f_{b}\right)+\epsilon_{1}+\epsilon_{2}\right]\left(1+f_{i t b}\right) \frac{\tau_{\lambda-i t b}}{\tau_{r}}\left(1-\tau_{t L}\right)$ 
Substituting equation (F.5) into (F.19), it yields:

$\tau_{f}=1+\left(\tau_{f R}-1\right) \eta_{m L} \frac{\tau_{\lambda-i t b}}{\tau_{r}}\left\{\frac{\left(1-\tau_{t L}\right)\left[\left(1-\beta-\epsilon_{1}-\epsilon_{2}\right)\left(1+f_{b}\right)+\epsilon_{1}+\epsilon_{2}\right]\left(1+f_{i t b}\right)}{\tau_{c L R}-1+\alpha\left(\tau_{f R}-1\right)}\right\}$

The LPC total temperature ratio follows from equation $(\mathrm{F.5})$ :

$$
\tau_{c L}=1+\left(\tau_{f}-1\right) \frac{\tau_{c L R}-1}{\tau_{f R}-1}
$$

Using LPC efficiency, the LPC total pressure ratio is given by:

$$
\pi_{c L}=\left\{1+\eta_{c L}\left(\tau_{c L}-1\right)\right\}^{\gamma_{c} /\left(\gamma_{c}-1\right)}
$$

From equation (9) of [28],

$$
\tau_{\lambda-i t b}=\frac{c_{p i t b} T_{t 4.5}}{c_{p c} T_{0}}
$$

Substituting equation (F.23) into Equation (F.20) yields

$$
\begin{aligned}
\tau_{f}= & 1+\left(\tau_{f R}-1\right) \eta_{m L} \frac{c_{p i t b} T_{t 4.5}}{c_{p c} T_{0}}\left(\frac{T_{0}}{T_{r e f} \theta_{0}}\right) \\
& \left\{\frac{\left(1-\tau_{t L}\right)\left[\left(1-\beta-\epsilon_{1}-\epsilon_{2}\right)\left(1+f_{b}\right)+\epsilon_{1}+\epsilon_{2}\right]\left(1+f_{i t b}\right)}{\tau_{c L R}-1+\alpha\left(\tau_{f R}-1\right)}\right\}
\end{aligned}
$$

Similar to the assumption in HPT, the flow is choked at station 4.5. Therefore, the value of $\tau_{t L}$ will remain constant. In addition, $f_{b}$ and $f_{i t b}$ are negligible when compared to unity.

Unfortunately, $\alpha$ is not a constant but a function of $\left(\pi_{c L}, \pi_{c H}, \pi_{f}, M_{18}\right)$. Let

$$
C_{2}=\left(\tau_{f R}-1\right) \eta_{m L} \frac{c_{p i t b}}{c_{p c}}\left(\frac{1}{T_{r e f}}\right)\left(1-\tau_{t L}\right)\left[\left(1-\beta-\epsilon_{1}-\epsilon_{2}\right)\left(1+f_{b}\right)+\epsilon_{1}+\epsilon_{2}\right]\left(1+f_{i t b}\right)
$$


Then,

$$
\tau_{f}=1+C_{2} \frac{T_{t 4.5}}{\theta_{0}}\left\{\frac{1}{\tau_{c L R}-1+\alpha\left(\tau_{f R}-1\right)}\right\}
$$

Substituting (F.26) into (F.21) yields

$$
\tau_{c L}=1+C_{3} \frac{T_{t 4.5}}{\theta_{0}}\left\{\frac{1}{\tau_{c L R}-1+\alpha\left(\tau_{f R}-1\right)}\right\}
$$

where

$$
C_{3}=\eta_{m L} \frac{c_{p i t b}}{c_{p c}}\left(\frac{1}{T_{r e f}}\right)\left(1-\tau_{t L}\right)\left[\left(1-\beta-\epsilon_{1}-\epsilon_{2}\right)\left(1+f_{b}\right)+\epsilon_{1}+\epsilon_{2}\right]\left(1+f_{i t b}\right)\left(\tau_{c L R}-1\right)
$$

Substituting (F.27) into (F.22) yields

$$
\pi_{c L}=\left[1+C_{4} \frac{T_{t 4.5}}{\theta_{0}}\left(\frac{1}{\tau_{c L R}-1+\alpha\left(\tau_{f R}-1\right)}\right)\right]^{\gamma_{c} /\left(\gamma_{c}-1\right)}
$$

where

$$
C_{4}=\eta_{c L} C_{3}
$$

Again, when compared to equation (D.3) of [34], $\pi_{c L}$ is not only a function of throttle setting $\left(T_{t 4}\right)$ and flight conditions $\left(\theta_{0}\right)$, but also a function of $\alpha$ :

$$
\pi_{c L}=f\left(\frac{T_{t 4.5}}{\theta_{0}}, \alpha\right)
$$

\section{F.5 Limiting Procedures}

\section{F.5.1 Reference Conditions}

The values of $T_{t 4 \max }$ and $T_{t 4.5 \max }$ (control-limit variables) for a turbofan engine are always equal to or smaller than the values of $T_{t 4 R}$ and $T_{t 4.5 R}$ at its reference-point (or on-design) conditions. 


\section{F.5.2 Limiting $T_{t 4}$ and $T_{t 4.5}$}

As shown in Figure F.1, as $\theta_{0}$ is decreased to the left of the theta break, or $\theta_{\text {obreak }}, T_{t 4}$ must be reduced in order to protect the compressor from poor operation. By doing so, the term $\frac{T_{t 4}}{\theta_{0}}$ must remains constant.

A simple limiting procedure as shown below is included into the code in order to limit $T_{t 4}$ values so that it is always equal to or less than $T_{t 4 \max }$.

$$
\theta_{0}=\tau_{r} \frac{T_{0}}{T_{r e f}}
$$

If $\left(\theta_{0} \geq \theta_{0 R}\right)$ then

$$
T_{t 4}=T_{t 4 \max }
$$

Else

$$
T_{t 4}=T_{t 4 \max } \frac{\theta_{0}}{\theta_{0 R}}
$$

When ITB is considered, a separate limiting procedure is required to limit $T_{t 4.5}$ values so that it is equal to or less than $T_{t 4.5 \text { max }}$.

$$
\begin{aligned}
& \text { If }\left(\theta_{0} \geq \theta_{0 R}\right) \text { then } \\
& \qquad T_{t 4.5}=T_{t 4.5 \max }
\end{aligned}
$$

Else

$$
T_{t 4.5}=T_{t 4.5 \max } \frac{\theta_{0}}{\theta_{0 R}}
$$

\section{F.5.3 Limiting $\pi_{f}$ and $\pi_{c H}$}

For a two-spool turbofan engine with ITB, there are two engine fuel flows. The fuel flow to the main burner directly influences the speed of the HP spool and the HPC pressure ratio. The fuel flow to the ITB directly influences the speed of the LP spool, the fan pressure ratio, and LPC pressure ratios. 


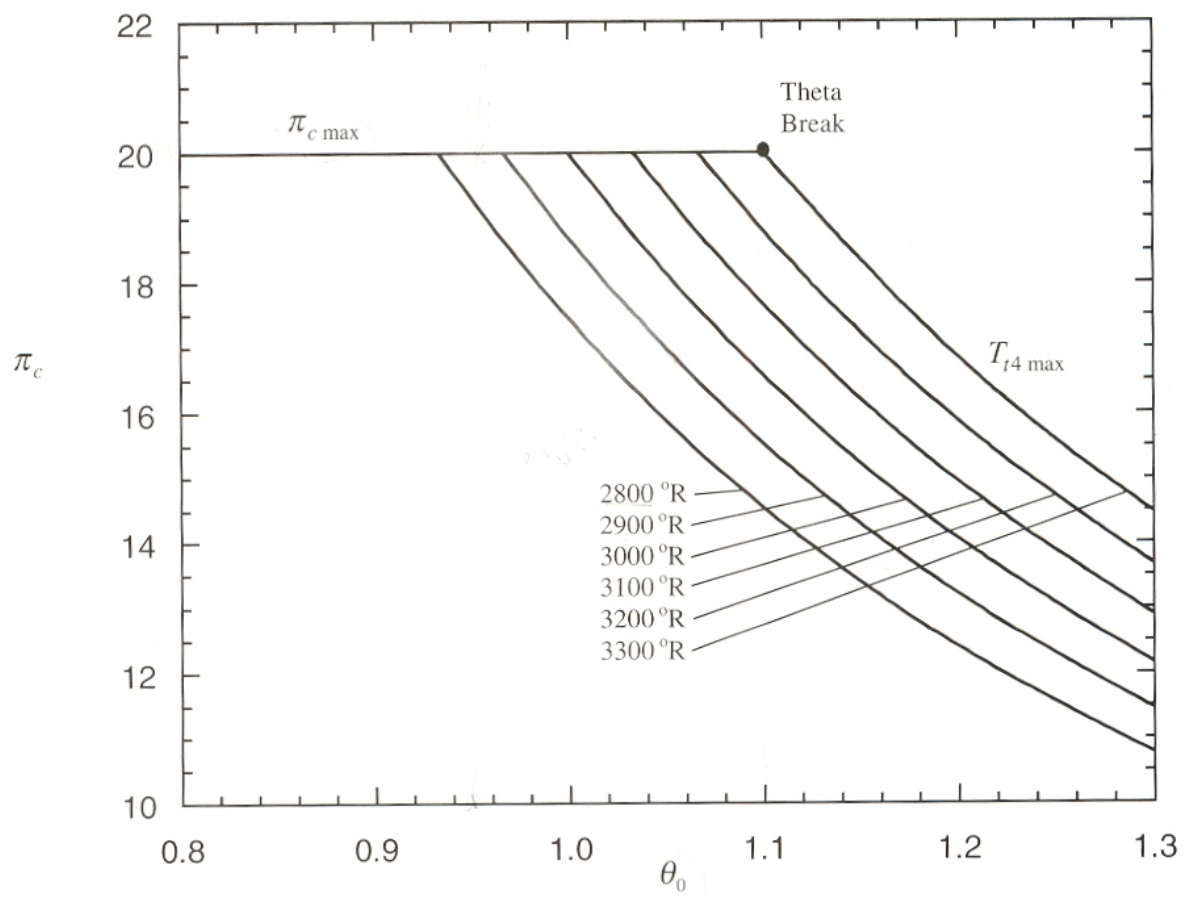

Figure F.1: CPR as a function of $\delta_{0}$ and $T_{t 4}$ for a compressor with a reference point of $\pi_{c}=\pi_{c \max }=20, T_{t 4}=T_{t 4 \max }=3300 \circ R(1833 \mathrm{~K})$, and $\delta_{0}=1.1$. 334

In Full Throttle Performance (FTP), when HPC pressure ratio is greater than its given maximum, the actual engine control will reduce the required fuel flow to the main burner in real time until the given limit is reached. In this study, the code does not calculate the reduction in fuel flow directly. Instead, the code will reduce the values of $T_{t 4}$ (main burner) or $T_{t 4.5}$ (ITB) to a lower value.

Therefore, two control limits have been selected in FTP, i.e., fan pressure ratio, $\pi_{f \max }$, for the LP spool, and HPC pressure ratio (HPCPR), $\pi_{c H \max }$, for the HP spool. Based on this, two throttle control loops have been added to the code as shown in Table F.1.

When the FPR is greater than its limit, $\pi_{f \max }$ (case 1 ), the control loop will reduce $T_{t 4.5}$ (i.e.,reduce fuel flow to the ITB). When the HPCPR is greater than its limit, $\pi_{c H \max }\left(\right.$ case 2 ), the control loop will reduce $T_{t 4}$ (reduce fuel flow 
to the main burner).

If ITB is on, the control loops for FPR and HPCPR are sequential, i.e., FPR limit control goes first, followed by HPCPR limit control.

If the ITB is off and the FPR is still greater than its limit, $\pi_{f \max }$ (case 3 ), the control loop will reduce $T_{t 4}$ (reduce fuel flow to the main burner). This reduction in $T_{t 4}$ will reduce both FPR and HPC. Similarly, this reduction in $T_{t 4}$ applies to a case where the FPR is still exceeding $\pi_{f \max }$ even when $T_{t 4.5}$ is reduced until the ITB is turned off (i.e., case 1 shifted to 3 ).

Table F.1: The Logic sequence of reducing temperature (reducing fuel flow to the burners)

\begin{tabular}{|c|c|c|c|c|}
\hline & \multicolumn{2}{|c|}{ Reduce $T_{t 4.5}$ when } & \multicolumn{2}{c|}{ Reduce $T_{t 4}$ when } \\
\hline Case & $\pi_{f} \geq \pi_{f \max }$ & $\pi_{c H} \geq \pi_{c H \max }$ & $\pi_{f} \geq \pi_{f \max }$ & $\pi_{c H} \geq \pi_{c H \max }$ \\
\hline ITB on & 1 & - & - & 2 \\
\hline ITB off & - & - & 3 & 4 \\
\hline
\end{tabular}

\section{F.5.4 Numerical Method for Reducing Temperature}

In this study, the adjustment of throttle settings is done by simply reducing the value of exit temperature of each burner. It is assumed that the fuel flow to each burner is directly proportional to the specified exit burner temperature.

While the control variable is exceeding its maximum allowable limit, the engine control subroutine will automatically reduce the corresponding exit burner temperature as a mean of reducing the fuel flow to the engine. Let the $Q$ be the control variable, $T_{t}$ be the exit burner temperature and $n$ be the current iteration, The following expressions show a step-by-step numerical algorithm for reducing the temperature: 


$$
\begin{aligned}
\Delta Q^{n} & =Q^{n-1}-Q^{n} \\
\Delta T_{t}^{n} & =T_{t}^{n-1}-T_{t}^{n} \\
Q^{n-1} & =Q^{n} \\
T_{t}^{n-1} & =T_{t}^{n} \\
\Delta T_{t}^{n+1} & =\left(Q^{n}-Q_{\max }\right) \frac{\Delta T_{t}^{n}}{\Delta Q^{n}} \\
i f(a b s & \left.\left.\Delta T_{t}^{n+1}\right)>100\right) \quad \text { then } \quad \Delta T_{t}^{n+1}=\frac{\Delta T_{t}^{n+1}}{\operatorname{abs}\left(\Delta T_{t}^{n+1}\right)} \times 100 \\
T_{t}^{n+1} & =T_{t}^{n}-\Delta T_{t}^{n+1}
\end{aligned}
$$

Obviously, the maximum reduction in $T_{t}$ at once is currently set to 100 .

In addition, Table F.2 gives a summary of all the control variables and its location in the code. Except for $T_{t 4}$ and $T_{t 4.5}$, all other control variables (i.e., $\pi_{f}, \pi_{c H}, P_{t 3}, T_{t 3}, \% N_{L}$, and $\left.\% N_{H}\right)$ utilize the algorithm described above for the reduction of $T_{t}$. The reduction mechanism for the control variables $T_{t 4}$ and $T_{t 4.5}$ have already been introduced in Section F.5.2.

Table F.2: Summary of the engine control variables $Q$ and its location in the code.

\begin{tabular}{|c|c|c|}
\hline Limit & Control Variable $Q$ & Subroutine \\
\hline 1 & $T_{t 4}$ & ctrlimit \\
\hline 2 & $T_{t 4.5}$ & ctrlimit \\
\hline 3 & $\pi_{f}$ & itbperf2 \\
\hline 4 & $\pi_{c H}$ & itbperf2 \\
\hline 5 & $P_{t 3}$ & itbperf2 \\
\hline 6 & $T_{t 3}$ & itbperf2 \\
\hline 7 & $\% N_{L}$ & itbperf2 \\
\hline 8 & $\% N_{H}$ & itbperf2 \\
\hline
\end{tabular}




\section{Appendix G}

\section{User Guide to the Parametric Cycle Analysis Code}

The Excel program is written in combination between spreadsheet neuron cells, visual basic, and macrocode. These three combinations provide userfriendly software that compilation and preprocessing are no longer necessary. The input is to well-labeled Excel cells. Most of the values are pre-specified and an example case is displayed. It is good practice to save this case by another name before modifying the spread sheet in order to retrieve the starting case. User obtains result plots right a way just by clicking some simple buttons.

\section{G.1 Definitions}

First of all, it is necessary for users to understand some icons that appear in the Excel sheets, for example, the 'Input' sheet as shown in Figure G.1. Table G.1 shown in next page lists down all the icons along with their descriptions. 


\section{G.2 Program outline}

The program mainly comprises seven types of sheets, namely 'CoverPage', 'Instructions', 'Input', plot sheets, data sheets, 'Singlept', and 'Station'. Meanwhile, the main code structure is shown in Figure I.1] in Appendix I.

\section{G.2.1 'CoverPage' sheet}

The 'CoverPage' sheet contains the information of authors of this program. Any questions regarding the program can be addressed to the corresponding author through email or by phone.

Table G.1: Different kinds of tools and indicators in cycle analysis codes

\begin{tabular}{|c|c|c|}
\hline Name & Appearance & Descriptions \\
\hline \multirow{2}{*}{ Combo box } & Unit & User change options through selecting \\
\hline & SI & the items in the pull down menu \\
\hline Button & Calculate & $\begin{array}{l}\text { Clicking any button will execute } \\
\text { the macrocode associated with it }\end{array}$ \\
\hline User Input & & $\begin{array}{l}\text { This is where users input the } \\
\text { values for variables }\end{array}$ \\
\hline Indicator 1 & & $\begin{array}{l}\text { The values here change with } \\
\text { altitude. DO NOT modify. }\end{array}$ \\
\hline Indicator 2 & & $\begin{array}{l}\text { All input values are read and shown } \\
\text { here. DO NOT modify. }\end{array}$ \\
\hline Indicator 3 & & $\begin{array}{l}\text { This is where reference engine data } \\
\text { are displayed. Users can modify if } \\
\text { necessary. (only in off-design code) }\end{array}$ \\
\hline
\end{tabular}

\section{G.2.2 'Instructions' sheet}

First time users are strongly recommended to read this sheet before running the program. Since there are always possibilities of getting error computations such as division by zero, square root of a negative quantity, or over floating, and under floating number, the program is written such that it will not crash if those errors are encountered during the computation. It will instead tell the user where 
the computation encounters those errors. In this section, you will find the details of how to run the program and how to fix a problem if something goes wrong.

This sheet also explains several assumptions made in the equations so that the users aware of some cases in the equations that have been idealized to simplify the problems.

\section{G.2.3 'Input' sheet}

This sheet is where most of the inputs are specified. The program will check input values in this sheet to make sure that all the inputs are specified. It will tell the users if there are inputs that have not been specified.

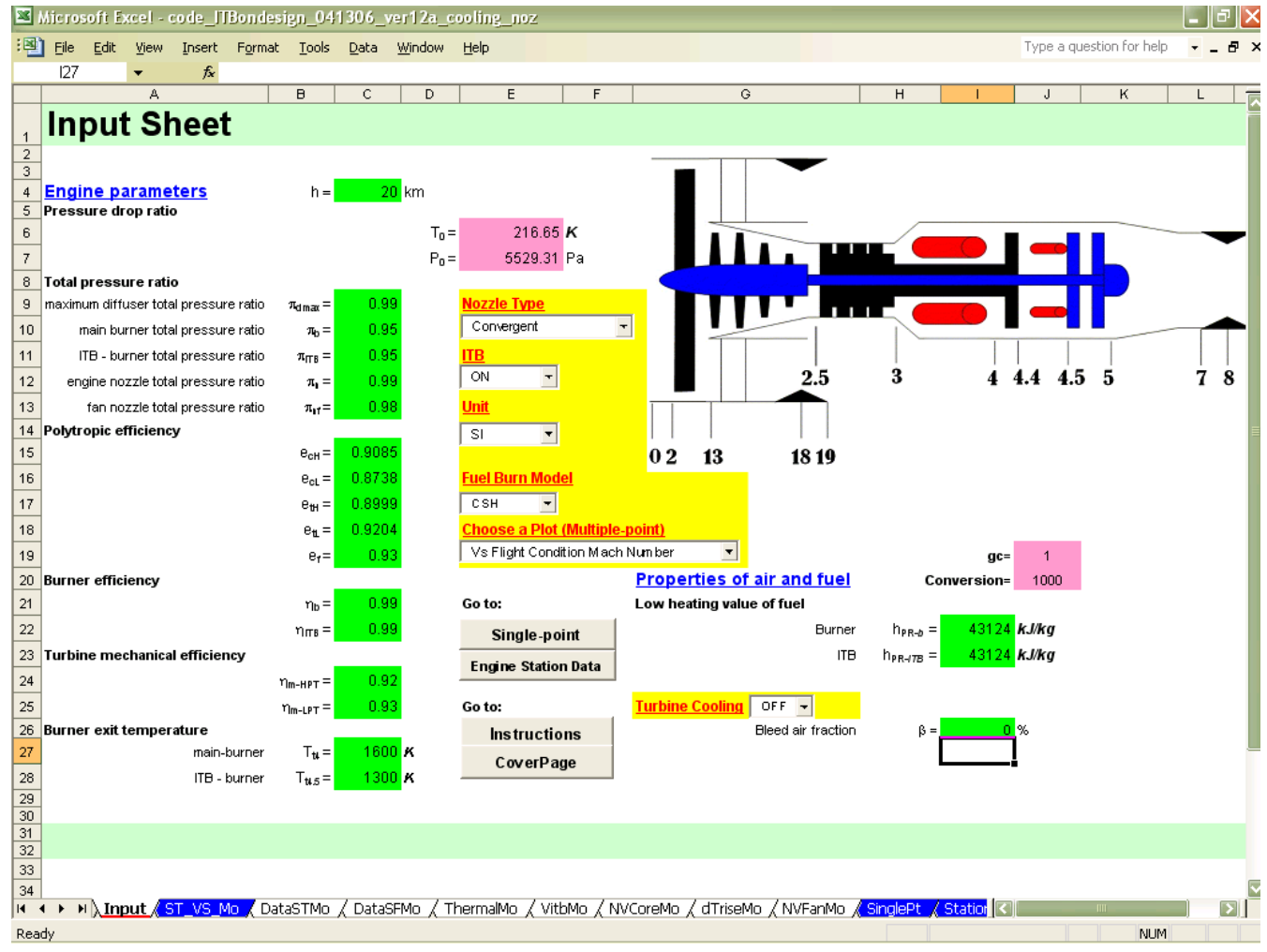

Figure G.1: A snapshot of 'Input' sheet in on-design cycle analysis code

There are four Combo Boxes in the 'Input' sheet (Combo Box is a list box that displays a list of values and lets the users select one of the value in the 
list), namely ITB, Unit, Fuel Burn Model, and Choose a Plot, as shown in Figure G.1. You need first to specify the option in these combo boxes, such as ITB, Unit and Fuel Burn Model, before moving on to combo box Choose a Plot.

Combo Box Unit lets you specify the unit system. Currently, the program can handle two unit systems, i.e., British gravitational units (English) and International system of units (SI).

Combo Box ITB lets you turn ON and OFF the ITB. This feature provides a flexibility to choose two types of engine and they are engine with ITB-ON and engine with ITB-OFF. With this feature, you will be able to see how the engine performance behaves with ITB-ON and with ITB-OFF.

Combo Box Fuel Burn Model let user choose two models for the computation of the fuel-air-ratio inside the burner, i.e., Constant Specific Heat (CSH) model and the Modified Specific Heat (MSH) model.

Once all the input values and the options in combo box ITB, Unit, and Fuel Burn Model are specified, you can specify an option in combo box Choose a Plot. This combo box provides several options to choose from, and they are:

1. Vs Compressor Pressure Ratio

2. Vs Flight Condition Mach Number

3. Vs Fan Pressure Ratio

4. Vs ByPass Ratio

5. Vs Main Burner Exit Temperature

In each option, it performs multiple calculations at different values of only 
one corresponding design variable. Once you select one of them, you will be directed to the relevant plot sheet.

\section{G.2.4 Plot sheets}

Please refer to section G.3 Multi-point Calculations for detailed information.

\section{G.2.5 Data sheets}

Please refer to section G.3 Multi-point Calculations for detailed information.

\section{G.2.6 'Singlept' sheet}

Please refer to section G.4 Single-point Calculation for detailed information.

\section{G.2.7 'Station' sheet}

Please refer to section G.5 Engine Station for detailed information.

\section{G.3 Multiple-point calculations}

The following discussion describes the instructions on how to run the program for each option available in combo box Choose a Plot in 'Input' sheet. Each option shares the similar code structure as shown in Figure I.2 in Appendix I.

\section{G.3.1 "Vs Compressor Pressure Ratio"}

When this option is chosen, users will be directed to a plot sheet, namely 'ST_VS_PIc', as shown in Figure G.2. This sheet is used to plot engine 
performances (e.g., specific thrust or thrust specific fuel consumption) and other properties (e.g., overall efficiency, nozzle jet velocity ratios, overall efficiency, fuel-air ratio, ITB inlet velocity and temperature rise across ITB) versus compressor pressure ratio $\left(\pi_{c}\right)$ with different bypass ratio $(\alpha)$. In order to execute the code properly, users are required to follow those circled number 1 through 3 sequentially as shown in Figure G.2.

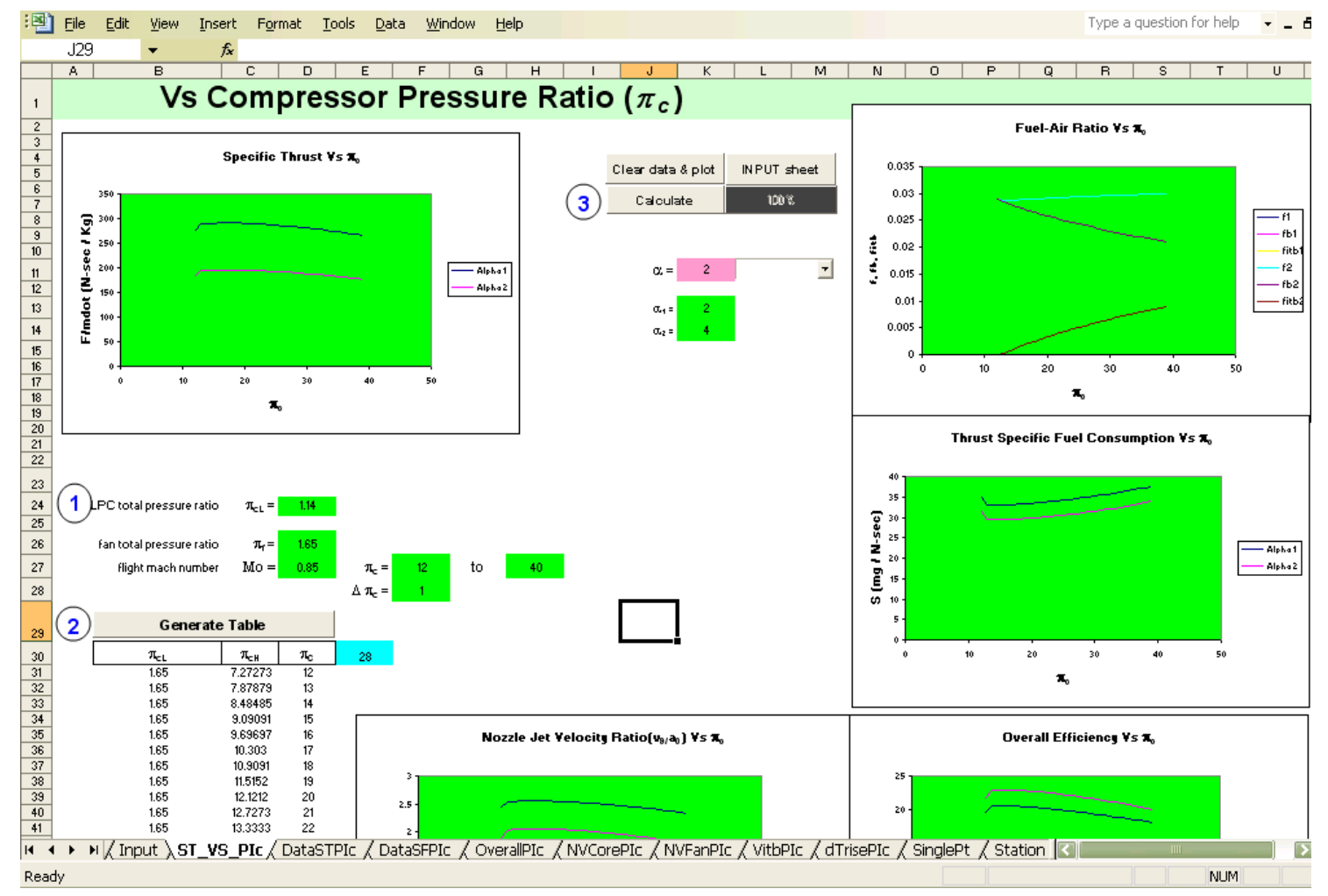

Figure G.2: A snapshot of "Vs Compressor Pressure Ratio" plot sheet in ondesign cycle analysis code

First of all, user needs to specify the values for three input parameters (i.e., $\pi_{c L}, \pi_{f}$, and $M_{0}$ ) as well as the lower and upper bound limit for "Compressor pressure ratio" $\left(\pi_{c}\right)$ and the increment $\left(\Delta \pi_{c}\right)$ (Figure G.3). For instance, the lower and upper bound limit are 12 and 40, respectively, whereas the increment is 1 .

What follows is to compute the values for HPC pressure ratio $\left(\pi_{c H}\right)$. By 


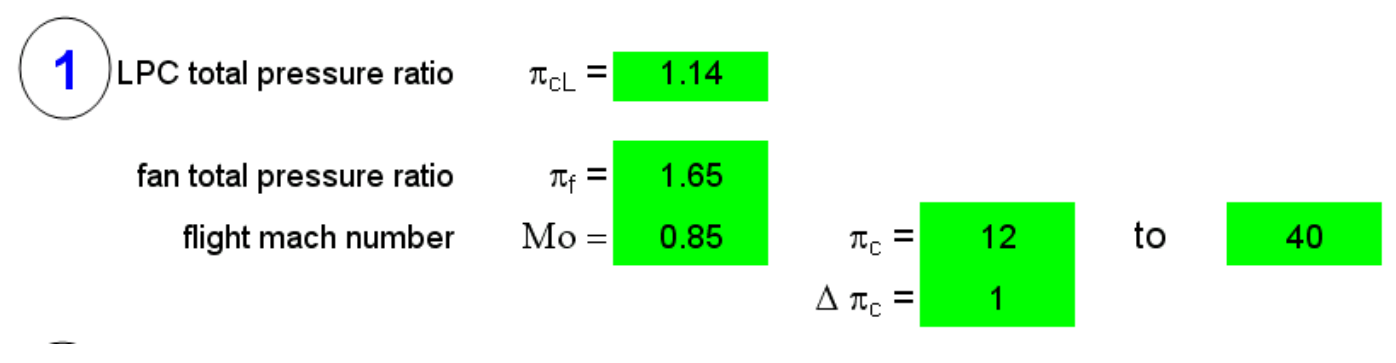

Figure G.3: Input parameters in "Vs Compressor Pressure Ratio" plot sheet in on-design cycle analysis code

\begin{tabular}{|c|c|c|c|}
\hline \multicolumn{3}{|c|}{ Generate Table } & \multirow[b]{2}{*}{28} \\
\hline$\pi_{\mathrm{CL}}$ & $\pi_{\mathrm{CH}}$ & $\pi_{\mathrm{C}}$ & \\
\hline 1.65 & 7.272727 & 12 & \\
\hline 1.65 & 7.878788 & 13 & \\
\hline 1.65 & 8.484848 & 14 & \\
\hline 1.65 & 9.090909 & 15 & \\
\hline 1.65 & 9.69697 & 16 & \\
\hline 1.65 & 10.30303 & 17 & \\
\hline 1.65 & 10.90909 & 18 & \\
\hline 1.65 & 11.51515 & 19 & \\
\hline 1.65 & 12.12121 & 20 & \\
\hline 1.65 & 12.72727 & 21 & \\
\hline 1.65 & 13.33333 & 22 & \\
\hline 1.65 & 13.93939 & 23 & \\
\hline 1.65 & 14.54545 & 24 & \\
\hline 1.65 & 15.15152 & 25 & \\
\hline
\end{tabular}

Figure G.4: Tabular data for compressor pressure ratio

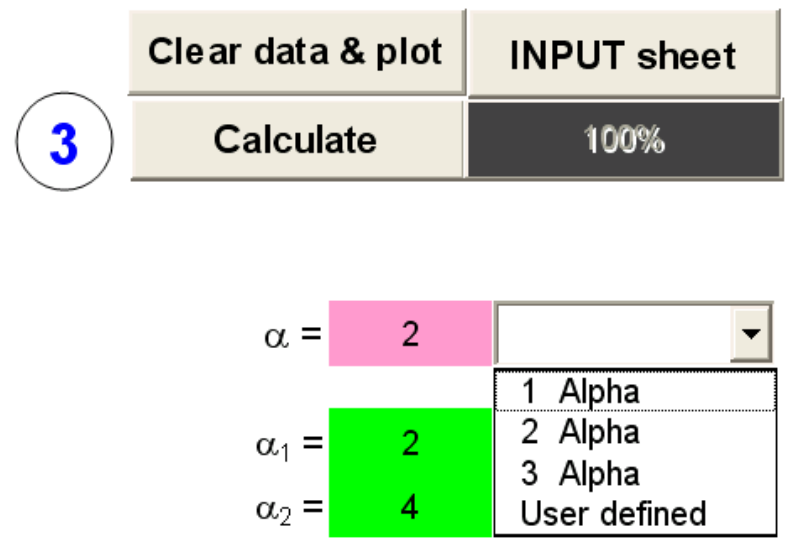

Figure G.5: Pull-down menu for specifying fan bypass ratios $(\alpha)$ 
clicking the Generate button, a tabular data for the compressor (including $\pi_{c L}$, $\pi_{c H}$, and $\pi_{c}$ ) will be listed as shown in Figure G.4. The cyan cell to the right of $\pi_{c}$ indicates the total number of iteration for $\pi_{c}$. The smaller the value for $\Delta \pi_{c}$, the larger the total number of $\pi_{c}$; and, therefore, more calculation at different values of $\pi_{c}$.

Thirdly, users are free to choose the number of bypass ratio $(\alpha)$ by selecting options from the pull down menu as shown in Figure G.5. If it is more than 3 $\alpha$ s, users are required to manually input any number greater than 3 by selecting 'User-Defined' option.

At this point, users are ready to run the code and generate the plots, simply by clicking Calculate button. All computed data will be tabulated in seven separate data sheets, namely, 'DataSTPIc', 'DataSFPIc', 'OverallPIc', 'NVCorePIc', 'NVFanPIc', 'VitbPIc', and 'dTrisePIc'.

Users are advised not to modify or change values in any of these data sheets. Clearing data as well as plots can be done by clicking Clear data \& plot button (Figure G.5).

To return to Input sheet for other runs with different design parameters, users simply click on 'Input' sheet button next to Calculate button.

\section{G.3.2 "Vs Flight Condition Mach Number"}

When users choose this option, the program hides the plot sheets and data sheets from the previous use and shows a associated plot sheet (i.e., 'ST_VS_Mo') and seven associated data sheets (i.e., 'DataSTMo', 'DataSFMo', 'OverallMo', 'NVCoreMo', 'NVFanMo', 'VitbMo', and 'dTriseMo'.) Users will then be directed from 'Input' sheet to 'ST_VS_Mo' sheet in a second. Similarly, this sheet is used to plot engine performances (e.g., 
specific thrust or thrust specific fuel consumption) and other properties (e.g., overall efficiency, nozzle jet velocity ratios, overall efficiency, fuel-air ratio, ITB inlet velocity, and temperature rise across ITB) versus flight Mach number $\left(M_{0}\right)$ with different bypass ratio.

Similar to the instructions for compressor pressure ratio, user first needs to specify the values for three input parameters (i.e., $\pi_{c L}, \pi_{f}$, and $\pi_{c}$ ) as well as the range of $M_{0}$ and the increment $\left(\Delta M_{0}\right)$.

Secondly, users are free to choose the number of $\alpha$ by selecting options from the pull down menu. At this point, users are ready to run the code and generate the plots, by simply clicking Calculate button. All computed data will be tabulated in all seven separate sheets just mentioned above.

\section{G.3.3 "Vs Fan Pressure Ratio"}

Similar to flight Mach number, this option opens a plot sheet (i.e., 'ST_VS_PIf') and seven data sheets (i.e., 'DataSTPIf', 'DataSFPIf', 'OverallPIf', 'NVCorePIf', 'NVFanPIf', 'VitbPIf', and 'dTrisePIf'). It

plots engine performances (e.g., specific thrust or thrust specific fuel consumption) and other variables (e.g., overall efficiency, nozzle jet velocity ratios, overall efficiency, ITB inlet velocity, and temperature rise across ITB) versus fan pressure ratio $\left(\pi_{f}\right)$ with different bypass ratio. The instructions to run the program in this sheet are similar to those for flight Condition Mach number. (please refer to Section G.3.2

\section{G.3.4 "Vs ByPass Ratio"}

This option opens a plot sheet (i.e., 'ST_VS_Alp') and seven data sheets (i.e., 'DataSTAlp', 'DataSFAlp', 'OverallAlp', 'NVCoreAlp', 'NVFanAlp', 'VitbAlp', and 'dTriseAlp'). It plots engine performances (e.g., 
specific thrust or thrust specific fuel consumption) and other variables (e.g., overall efficiency, nozzle jet velocity ratios, overall efficiency, ITB inlet velocity, and temperature rise across ITB) versus different bypass ratio $(\alpha)$ with different fan total pressure ratios. The instructions to run the program in this sheet are analogous to those described above. The only difference is that selection of $\alpha$ is replaced by $\pi_{f}$.

\section{G.3.5 "Vs Main Burner Exit Temperature"}

This option opens a plot sheet (i.e., 'ST_VS_Tt4') and seven data sheets (i.e., 'DataSTt4', 'DataSFTt4', 'OverallTt4', 'NVCoreTt4', 'NVFanTt4', 'VitbTt4', and 'dTriseTt4'). It plots engine performances (e.g., specific thrust or thrust specific fuel consumption) and other variables (e.g., overall efficiency, nozzle jet velocity ratios, overall efficiency, ITB inlet velocity, and temperature rise across ITB) versus main burn exit temperature $\left(T_{t 4}\right)$ with different flight Mach number. The instructions to run the program in this sheet are similar to those for flight Condition Mach number. (See Section G.3.2)

\section{G.3.6 Summary}

In summary, you need to do the followings to run the program:

1. Go to the 'Input' sheet

2. Specify the Unit system (SI or English)

3. Specify the ITB switch. Is the computation for engine with ITB-ON or for engine with ITB-OFF?

4. Specify the Fuel Burn Model system (CSH or MSH)

5. Enter all the input parameters indicated in green cells (do not modify or change the value indicated in cyan). 
6. Specify Choose a Plot. You will be directed to a new sheet depending on the selection.

In sheet 'ST_VS_PIc' (Compressor Pressure Ratio),

1. Specify number of bypass ratio $(\alpha)$.

2. Specify all input parameters in green cells.

3. Click Generate to generate HPC total pressure ratio.

4. Click Calculate to compute and plot the results.

5. Repeat the above steps for different input parameters. To return to 'Input' sheet, simply click Input sheet button.

In sheet 'ST_VS_Mo' (Flight Condition Mach Number),

1. Specify number of bypass bypass ratio $(\alpha)$.

2. Specify all input parameters in green cells.

3. Click Calculate button.

4. Repeat the above steps for different input parameters. To return to 'Input' sheet, simply click Input sheet button.

In sheet 'ST_VS_PIf' (Fan Pressure Ratio),

1. Specify number of bypass ratio $(\alpha)$.

2. Specify all input parameters in green cells.

3. Click Calculate to compute and plot the results.

4. Repeat the above steps for different input parameters. To return to 'Input' sheet, simply click Input sheet button.

In sheet 'ST_VS_Alp' (ByPass Ratio), 
1. Specify number of fan total pressure ratio $\left(\pi_{f}\right)$.

2. Specify all input parameters in green cells.

\section{Click Calculate.}

4. Repeat the above steps for different input parameters. To return to 'Input' sheet, simply click Input sheet button.

In sheet 'ST_VS_Tt4' (Main Burner Exit Temperature),

1. Specify number of flight Mach number $\left(M_{0}\right)$.

2. Specify all input parameters in green cells.

3. Click Calculate button.

4. Repeat the above steps for different input parameters. To return to 'Input' sheet, simply click Input sheet button.

\section{G.4 Single Point Calculation}

All instructions described in previous section are termed as the multi-point calculations at different values of only one design parameter (e.g., $\pi_{c}, \pi_{f}, M_{0}, \alpha$, or $T_{t 4}$ ) The computed results show how the performance of a family of engines was determined by engine design choices and flight Mach number.

Once the most promising engine is selected by users (i.e., all engine components are fixed, e.g., $\pi_{c}, \pi_{f}$, altitude, $\alpha$ etc), single point calculation is then run to get the engine performance at this particular operating conditions. These output data represents the selected engine's design-point or reference-point condition. In order to predict the engine performance at off-design conditions, these outputs are then used as the reference conditions in an off-design code. This step is important because different reference conditions (i.e., different 


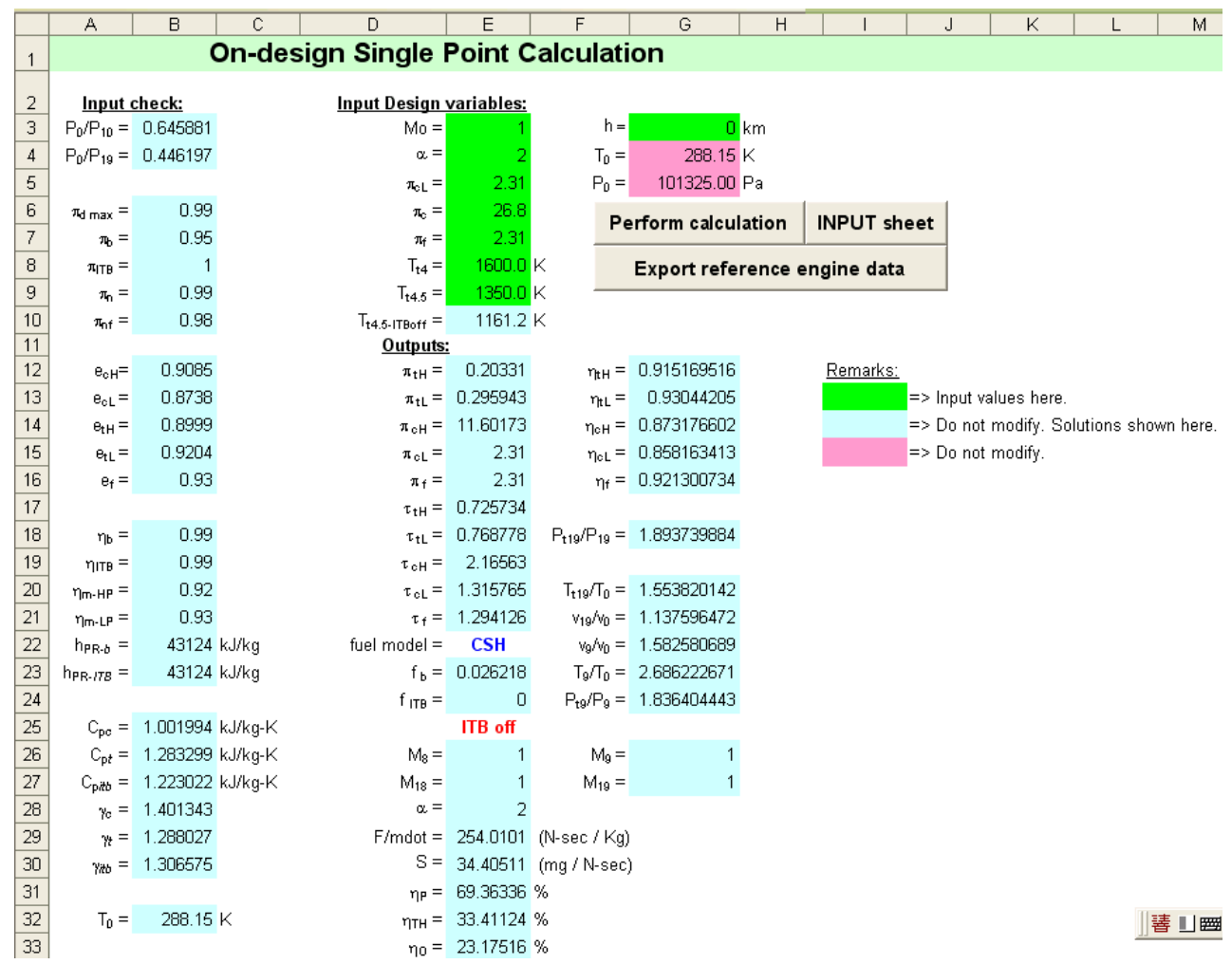

Figure G.6: On-design single point calculation sheet

engines) will give different performance over the whole flight spectrum.

For user's reference, the flow chart of this computation is shown in Figure I.3 in Appendix I.

\section{G.4.1 Exporting Reference Engine Data}

For convenience, authors include 'Input/Output files' feature in this code. The output data from this on-design code can be exported to be a reference engine data file, which is required in off-design calculation. As a result, users do not need to manually re-input the reference conditions in off-design code, which is a time-consuming process. The detailed outputs of this reference-point engine data format is shown in Figure G.7.

Users are to follow the step 1 to 13 to generate a reference engine data file: 


\begin{tabular}{|c|c|c|c|c|c|}
\hline Line & \begin{tabular}{|l|} 
Description \\
\end{tabular} & Remarks & Line & \begin{tabular}{|l|} 
Description \\
\end{tabular} & Remarks \\
\hline 1 & Unit System & \multirow{32}{*}{$\begin{array}{l}\text { ITB-on or -off } \\
\text { depending on } \\
\text { the user's } \\
\text { choice }\end{array}$} & 33 & $\eta_{\mathrm{tL}}$ & \multirow{21}{*}{$\begin{array}{l}\text { ITB-on or -off } \\
\text { depending on } \\
\text { the user's choice }\end{array}$} \\
\hline 2 & ITB & & 34 & $\eta_{\mathrm{cH}}$ & \\
\hline 3 & Cooling & & 35 & $\eta_{\mathrm{cL}}$ & \\
\hline 4 & Fuel model & & 36 & $\eta_{\mathbf{f}}$ & \\
\hline 5 & Nozzle type & & 37 & $\mathrm{P}_{0} / \mathrm{P}_{9}$ & \\
\hline 6 & $M_{0 R}$ & & 38 & $\mathrm{P}_{0} / \mathrm{P}_{19}$ & \\
\hline 7 & $h_{R}$ & & 39 & $\pi_{d \max }$ & \\
\hline 8 & $T_{t 4 R}$ & & 40 & $\boldsymbol{\pi}_{\mathbf{b}}$ & \\
\hline 9 & $T_{t 4.5 R}$ & & 41 & $\pi_{\text {itb }}$ & \\
\hline 10 & $\pi_{\text {thR }}$ & & 42 & $\pi_{n}$ & \\
\hline 11 & $\pi_{\text {tLR }}$ & & 43 & $\pi_{n f}$ & \\
\hline 12 & $\pi_{\text {cHR }}$ & & 44 & $\eta_{\mathbf{b}}$ & \\
\hline 13 & $\pi \mathrm{cLR}$ & & 45 & $\eta_{\text {itb }}$ & \\
\hline 14 & $\pi_{\mathrm{fR}}$ & & 46 & $\eta \mathrm{mH}$ & \\
\hline 15 & $\tau_{\text {tHR }}$ & & 47 & $\eta \mathrm{mL}$ & \\
\hline 16 & $\tau_{\text {tLR }}$ & & 48 & $h_{\text {PR-b }}$ & \\
\hline 17 & $\tau_{\mathrm{cHR}}$ & & 49 & $\varepsilon_{1 \mathrm{R}}$ & \\
\hline 18 & $\tau_{\mathrm{cLR}}$ & & 50 & $\varepsilon_{2 \mathrm{R}}$ & \\
\hline 19 & $\tau_{\mathrm{fR}}$ & & 51 & $\beta$ & \\
\hline 20 & "space" & & 52 & $\tau_{\mathbf{m 1 R}}$ & \\
\hline 21 & $f_{b R}$ & & 53 & $\tau_{\mathrm{m} 2 \mathrm{R}}$ & \\
\hline 22 & $f_{\text {itbR }}$ & & 54 & $F_{R}$ & \multirow{11}{*}{ ITB-off condition } \\
\hline 23 & - & & 55 & $\mathrm{~S}_{\mathrm{R}}$ & \\
\hline 24 & $M_{8 R}$ & & 56 & $\eta_{\mathbf{P}}$ & \\
\hline 25 & $M_{18 R}$ & & 57 & $\eta_{\text {TH }}$ & \\
\hline 26 & $\alpha_{R}$ & & 58 & $\eta_{0}$ & \\
\hline 27 & $F_{R}$ & & 59 & $\pi$ tLR & \\
\hline 28 & $S_{R}$ & & 60 & $\tau_{\text {tLR }}$ & \\
\hline 29 & $\eta_{\mathbf{p}}$ & & 61 & $\eta_{\text {tL }}$ & \\
\hline 30 & $\eta_{\text {TH }}$ & & 62 & $f_{\text {itbR }}$ & \\
\hline 31 & $\eta_{0}$ & & 63 & $\pi_{\text {itb }}$ & \\
\hline 32 & $\eta_{\mathrm{tH}}$ & & 64 & $M_{8 R}$ & \\
\hline
\end{tabular}

Figure G.7: List of variables in the reference engine data file for use in off-design code. 
1. Select 'Input' sheet (as shown in Figure G.1).

2. Input the engine characteristics in 'Input' sheet.

3. Select 'SinglePt' sheet (as shown in Figure G.6).

4. Input values of independent variables (i.e., $M_{0}, \alpha$, altitude, $\pi_{c}$, and throttle settings) in green cells.

5. Click on Perform calculation button to calculate the selected engine performance at a design point.

6. Both the input values from 'Input' sheet and the solution will be shown in light-blue cells.

7. Click on Export reference engine data button to export these reference engine data to a text file.

8. A window dialog box will appear and request for specifying the path and filename.

9. Check for existing drive path or/and specify filename.

10. All reference data and engine characteristics of a specific design-point engine are successfully exported.

11. Repeat step 1 to 10 for different engines.

\section{G.5 Engine Station Data}

The last sheet in the program is the 'Station' sheet. This sheet displays the engine station flow properties (i.e., temperature and pressure) in forms of a table (Figure G.8) and charts (Figure G.9) based on the user input Mach number data at certain stations. 
Most of the input parameters are from the 'Input' sheet. Additional inputs are also needed, such as those listed in "Inputs" column (i.e., to the left of the engine station data table) and Mach numbers at some engine stations (Figure G.8). To click the Update Station Data button will update the flow properties at each engine station. Please refer to Figure I.4 in Appendix I for the code structure of this sheet.

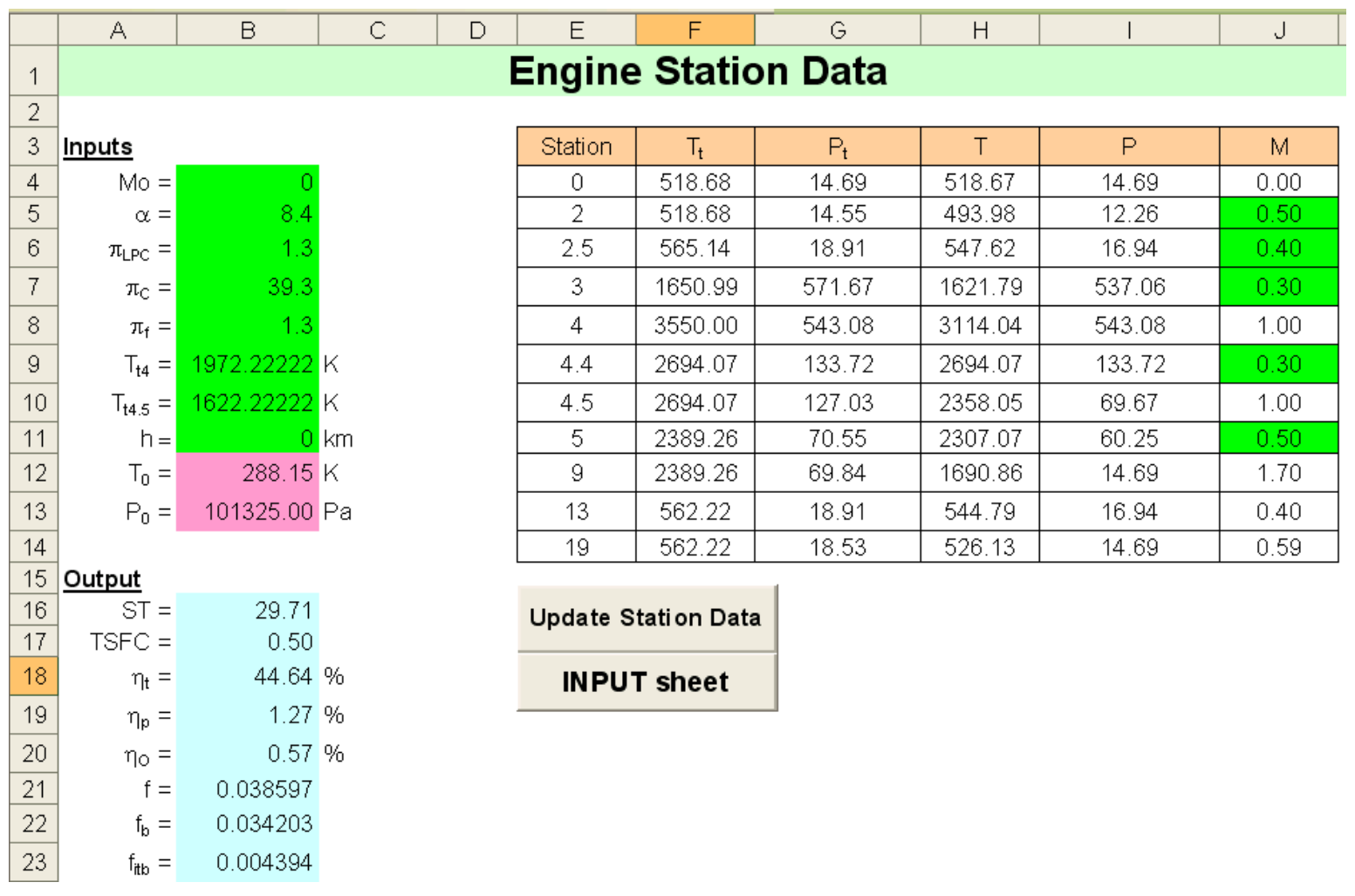

Figure G.8: On-design engine station data calculation sheet 


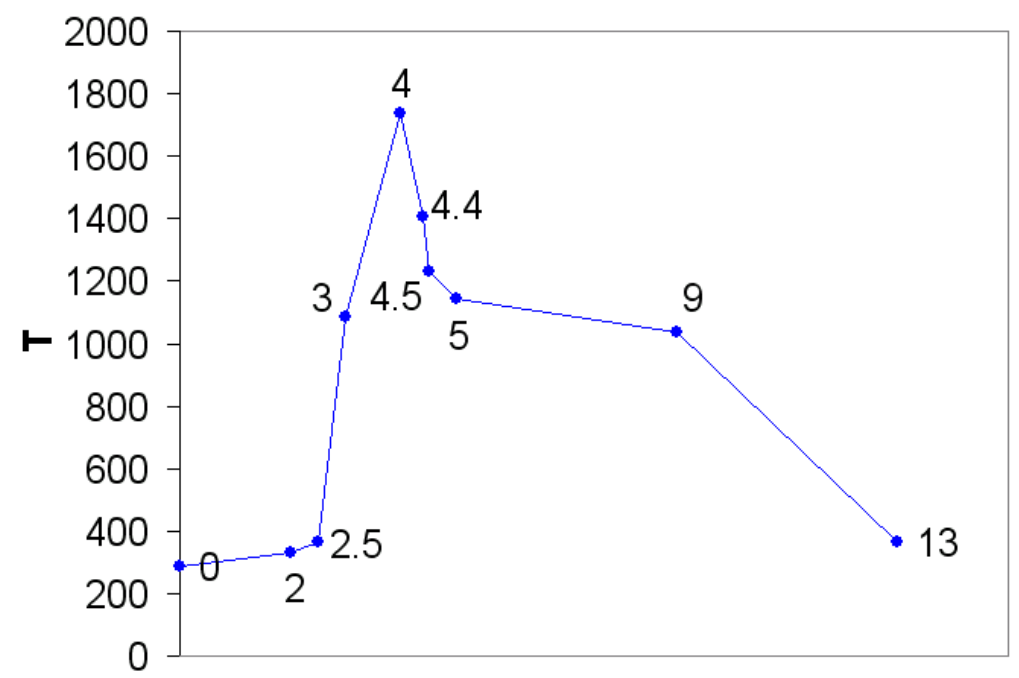

Station

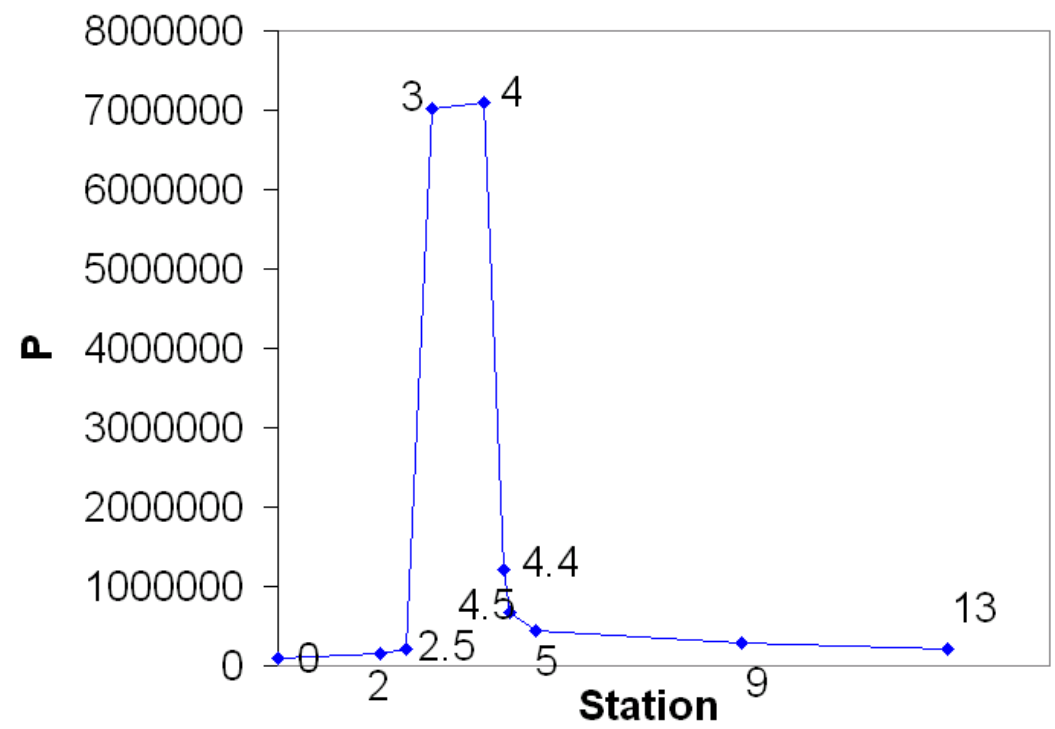

Figure G.9: Examples of temperature and pressure versus engine stations 


\section{G.6 Discussions}

A typical value for local Mach number at the ITB entrance is selected to be 0.3. This value $\left(M_{4.4}=0.3\right)$ is then used to compute ITB inlet velocity $\left(v_{4.4}\right)$, on which one of the factors ITB design was based. In fact, each plot sheet will show the change of ITB inlet velocity with the corresponding design parameter, for example, $v_{4.4}$ versus $M_{0}$ in "ST_VS_Mo" sheet.

At ITB-OFF condition, a plot of "Temperature Rise across ITB" in each plot sheet (e.g., 'ST_VS_PIc', 'ST_VS_Mo', 'ST_VS_PIf', and 'ST_VS_Alp') will be cleared out. It is because the ITB-OFF engine acts as if it is a turbofan engine without any increase across ITB.

\section{G.7 Troubleshooting}

This program has been debugged several times. Therefore, whenever you encounter computation errors due to either zero division or square root of a negative quantity, you will be notified by a pop up window indicating where the computation problem is. It will tell you on which equation that the computational error is encountered. If you have any comments or bug problems you encounter in the program, you can report them to us for further improvement. Details about the contact number can be found in the 'CoverPage' sheet of the program.

\section{G.7.1 Out of Range}

In multiple-point calculation, users may always see these pop-up windows as shown in Figs. G.10a and G.10b. There are two possibilities of having this error or warning messages:

1. The input values are incorrect. For instance, the main burner exit temperature $\left(T_{t 4}\right)$ may be too small for an engine to produce enough thrust. Please double check all input values and try again. 
2. During the multiple-point computation, the design variable may be out of range. It will happen, especially for these design variables such as flight Mach number, fan pressure ratio and bypass ratio. For instance, the specific thrust of an engine is approaching zero as the flight Mach number is 3. If the upper limit of flight Mach number is set to be greater than 3 (e.g., 5), the code will not be able to proceed at Mach number higher than 3 and thus give these warning messages.

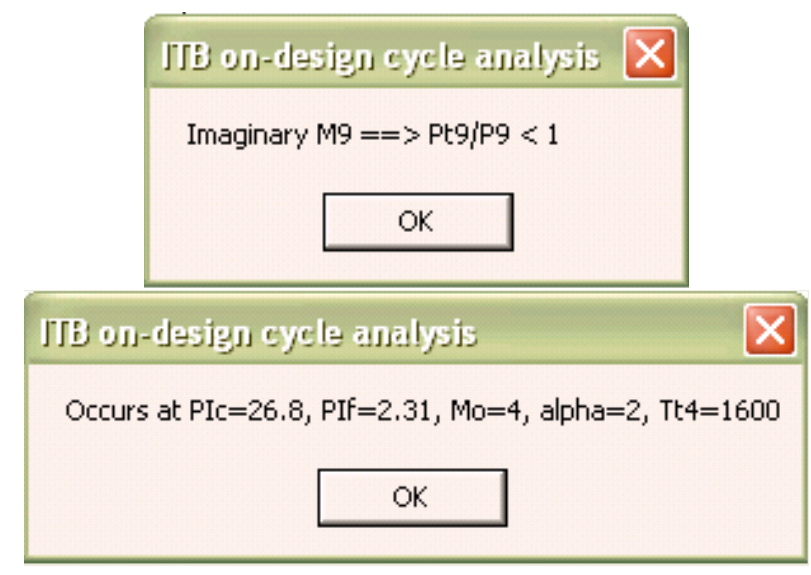

Figure G.10: Pop-up windows showing warning or error messages: (a) Equations where the error occurs; (b) The current values of design variables while error is encountered.

\section{G.7.2 Plotting errors}

It is also predicted that the plotting macro can encounter some problems in the future if the users do not fully understand the program. Therefore, authors have set up a way in order to fix the problem. In case the plot starts giving problem, you need to do the followings (this apply to all plot sheets: 'ST_VS_PIc', 'ST_VS_Mo', 'ST_VS_PIf', 'ST_VS_Alp', and 'ST_VS_Tt4'):

1. Go to the plot sheet (e.g., 'ST_VS_Mo').

2. Go to cell D5. 
3. Note that you will not be able to click on that cell because it is lying on the back of the plot chart. You may want to use arrow button from your keyboard in order to move to cell D5.

4. In that cell, you will see "SUCCESS". Change the character to "FAIL".

5. Clear the series in the chart plot by clicking right mouse button on the chart plot.

6. In right mouse button menu list, choose Source Data.

7. In 'Source Data' windows, pick Series tab and remove all the series.

Users are not expected to understand macro code in the program. However the part that takes care the computation can be found in Module1 Sub Itb(). To open Excel Visual Basic windows, users need to press Alt + F11. Any modification can be made in subroutine $I t b()$. Do not add new variables into the program. 


\section{Appendix $\mathbf{H}$}

\section{User Guide to the Performance Cycle Analysis Code}

Similar to parametric cycle code, the performance cycle code is written in combination between spreadsheet neuron cells, visual basic, and macro code. Most of the values are pre-specified and an example case is displayed. It is a good practice to save this case by another name before modifying the spread sheet in order to retrieve the starting case.

The program mainly comprises of three sections, namely, 'Input', 'Full Throttle Performance', and 'Partial Throttle Performance'. The Excel spreadsheets associated with each section are listed in the Table H.1 as shown below:

\section{H.1 Definitions}

All the icons that appear in the Excel sheets have been defined in Table G.1 and will not be repeated here. 


\section{H.2 User Inputs}

\section{H.2.1 'CoverPage' sheet}

The 'CoverPage' sheet contains the information about the authors of this program. Any questions regarding the program can be addressed to the corresponding author through the email or phone.

\section{H.2.2 'Instructions' sheet}

First-time users are strongly recommended to read this sheet before running the program.

In this sheet, you will find details of how to run the program. This sheet also explains several assumptions made in the equations so that the users are aware of some cases in the equations that have been idealized to simplify the problems.

Table H.1: List of Excel spreadsheets in off-design cycle code

\begin{tabular}{|c|l|c|l|}
\hline No. & \multicolumn{1}{|c|}{ Section } & Function & Excel spreadsheets \\
\hline \multirow{2}{*}{1.} & \multirow{2}{*}{ Input } & Introduction & Coverpage, Instruction \\
\cline { 3 - 4 } 2. & & Data Input & Input \\
\hline \multirow{2}{*}{$\begin{array}{l}\text { Full Throttle } \\
\text { Performance }\end{array}$} & Control & Control_FTP, SinglePt_FTP \\
\cline { 3 - 4 } 3. & $\begin{array}{l}\text { Partial } \\
\text { Throttle } \\
\text { Performance }\end{array}$ & Datput & $\begin{array}{l}\text { D_FTP, D_FvsM0, D_SvsM0, } \\
\text { D_picHvsM0, D_Tt4vsM0, } \\
\text { D_mdot0, D_pif, D_alp, D_Tt4p5. }\end{array}$ \\
\cline { 3 - 4 } & Data Output & Control_PTP \\
\hline
\end{tabular}

\section{H.2.3 'Input' sheet}

This sheet is where most of the inputs are specified. All cells in 'dark green' color (Indicator 1 as defined in Table G.1) need to be filled in with input values, except those cells in 'hotpink' color (Indicator 2 as defined in Table G.1), where the built-in values are set. The program will check all the inputs value in this 
sheet to make sure that the inputs are specified correctly. Window dialog boxes will pop up and notice the user if there are input values that are not specified or zero input values are found.

Once the design choice of a specific reference-point engine has been made from a parametric cycle analysis, the input values for the so-called Reference Conditions (i.e., the flight condition, throttle settings, engine component etc) and the 'Design Constants' are readily obtained. By clicking the Import data from ITB on-design code button, the code will automatically load the reference engine data file at a specified path and filename. All reference engine data will be displayed at all 'burlywood' cells (Indicator 3 as defined in Table G.1p. Some reference data are hidden at the back of the turbofan's schematic diagram. Detail of each variable's cell position is listed in Table J.6. These hidden data is read when the user to run an ITB-off case with an ITB-on reference-engine data. In addition, some data will be used to compare with the test engine data in single-point calculation. Besides importing reference engine data file, user can choose to manually input each input value, which is a time-consuming process.

There are four combo boxes in the 'Input' sheet (Combo Box is a list box that displays a list of values and lets the users select one of the value in the list), namely Unit System, ITB Switch, Fuel Burn Model, and Perform Computation in as shown in Figure H.1. You need first to specify the value in combo box Unit System, combo box ITB Switch, and combo box Fuel Burn Model before moving on to combo box Perform Computation in.

Combo box Unit System lets you specify the input and output unit system. Currently, the program can handle two units systems, namely, English and SI units. Combo box ITB Switch lets user to turn ON or OFF the ITB. This feature provides a flexibility to choose two types of engine and they are the engine 
with ITB-ON and with ITB-OFF. With this feature, you will be able to see how much engine performance you can get with ITB-ON and with ITB-OFF. Combo Box Fuel Burn Model lets user to choose two models for the computation of the fuel-air-ratio inside the burners, i.e., Constant Specific Heat (CSH) model and the Modified Specific Heat (MSH) model. For the details, please refer to Appendix C.

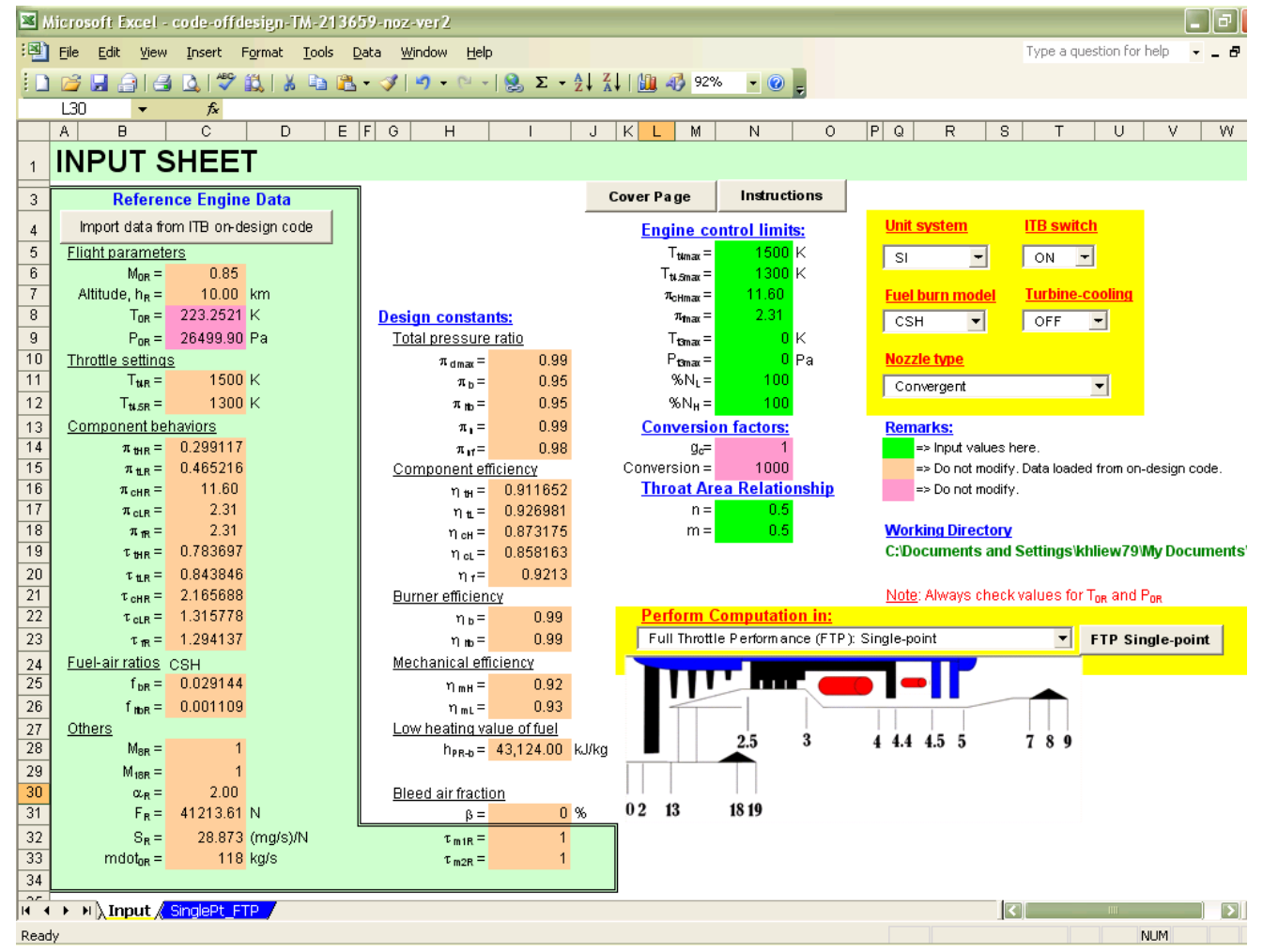

Figure H.1: A view output of 'Input' sheet in off-design cycle analysis code

Once all the inputs values are specified and all combo box values on Unit System, ITB Switch, and Fuel Burn Model are specified, user can specify a value in combo box Perform Computation in. This combo box provides three option lists to choose from, and they are:

1. Full Throttle Performance (FTP): Multiple-point

2. Full Throttle Performance (FTP): Single-point

3. Partial Throttle Performance (PTP) 
Once you select either one of them, you will be directed to some new sheets. The macro code associated with this combo box will open a relevant control sheet, and some hidden data sheets depending on the selection you make.

The following sections describe the instruction of how to run the program for each different option.

\section{H.3 Full Throttle Performance over M0 and Al- titude (FTP)}

\section{H.3.1 Multiple-point Calculations ('Control_FTP'sheet)}

When this option is chosen, the program opens several sheets, namely, one control sheet (i.e., either 'Control_FTP'), and nine data sheets (i.e. 'D_FTP', 'D_FvsM0', 'D_SvsM0', 'D_picHvsM0', 'D_Tt4vsM0', 'D_mdot0', 'D_pif', 'D_alp', and 'D_Tt4p5').

In 'Control_FTP' sheet, user expects to see a sheet as shown in Figure H.2. Before the computation, user needs to complete two input parameters, i.e., flight parameters and altitude, as indicated in 'dark green' cells. [NOTE: In this code, there is only one design variable, namely, flight Mach number. Other iteration variables, such as ambient conditions $\left(T_{0}\right.$ and $\left.P_{0}\right)$, altitude $(h)$, total exit temperature of main burner or ITB etc, may be added to the code with minor code modification in the future.]

To specify a range for flight Mach number $\left(M_{0}\right)$, user needs to input the values for the lower limit, upper limit, and an increment of $M_{0}$. If input lower limit of $M_{0}$ is zero, the code will internally change it from zero to 0.01 .

Click the Altitude $(h)$ combo box to select number of plots for different 


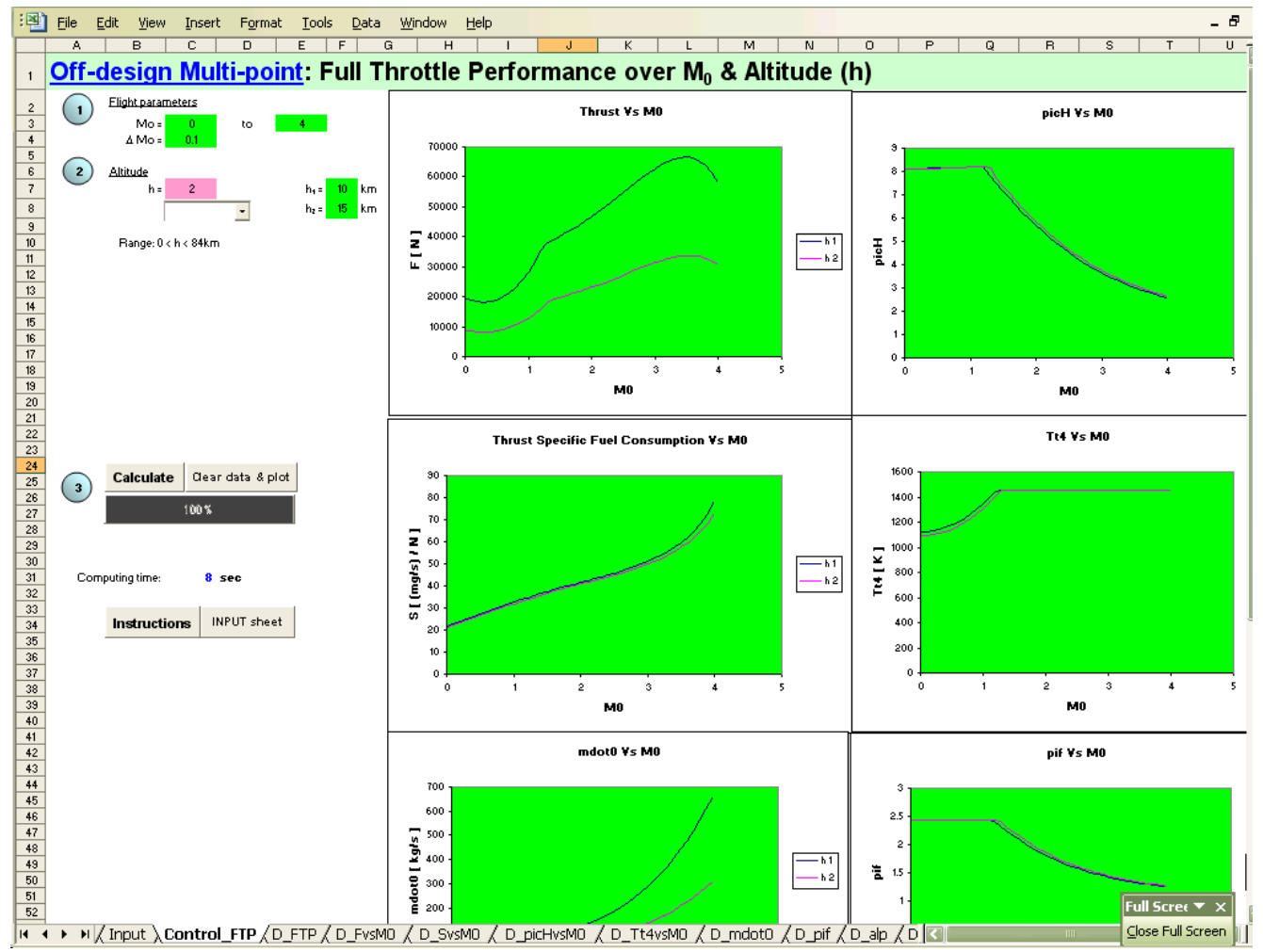

Figure H.2: Screenshot of Full-Throttle Performance: Multi-point calculations

altitudes. Selected number of cells will be created adjacent to the Altitude (h) combo box. Once all the 'dark green' cells are filled, it is ready to run the program and generate the plots, simply by clicking Calculate button. Data computed will be stored in nine data sheets.

Clicking the Clear data \& plot button will clear up all the computed solutions in data sheets as well as the eight plots in 'Control_FTP' sheet.

\section{H.3.2 Single-point Calculations ('SinglePt_FTP'sheet)}

When this option is chosen, the program opens one control sheet (i.e., 'SinglePt_FTP'). This option allows the user to predict the off-design performance of the selected engine at a fixed $M_{0}$ and a specified altitude. As indicated in Figure H.3, user just needs to specify a single value for each $M_{0}$ and altitude. Then, simply clicking the Calculate button will run the code. All the input 
values from 'Input' sheet and the solutions will be displayed in the same sheet.

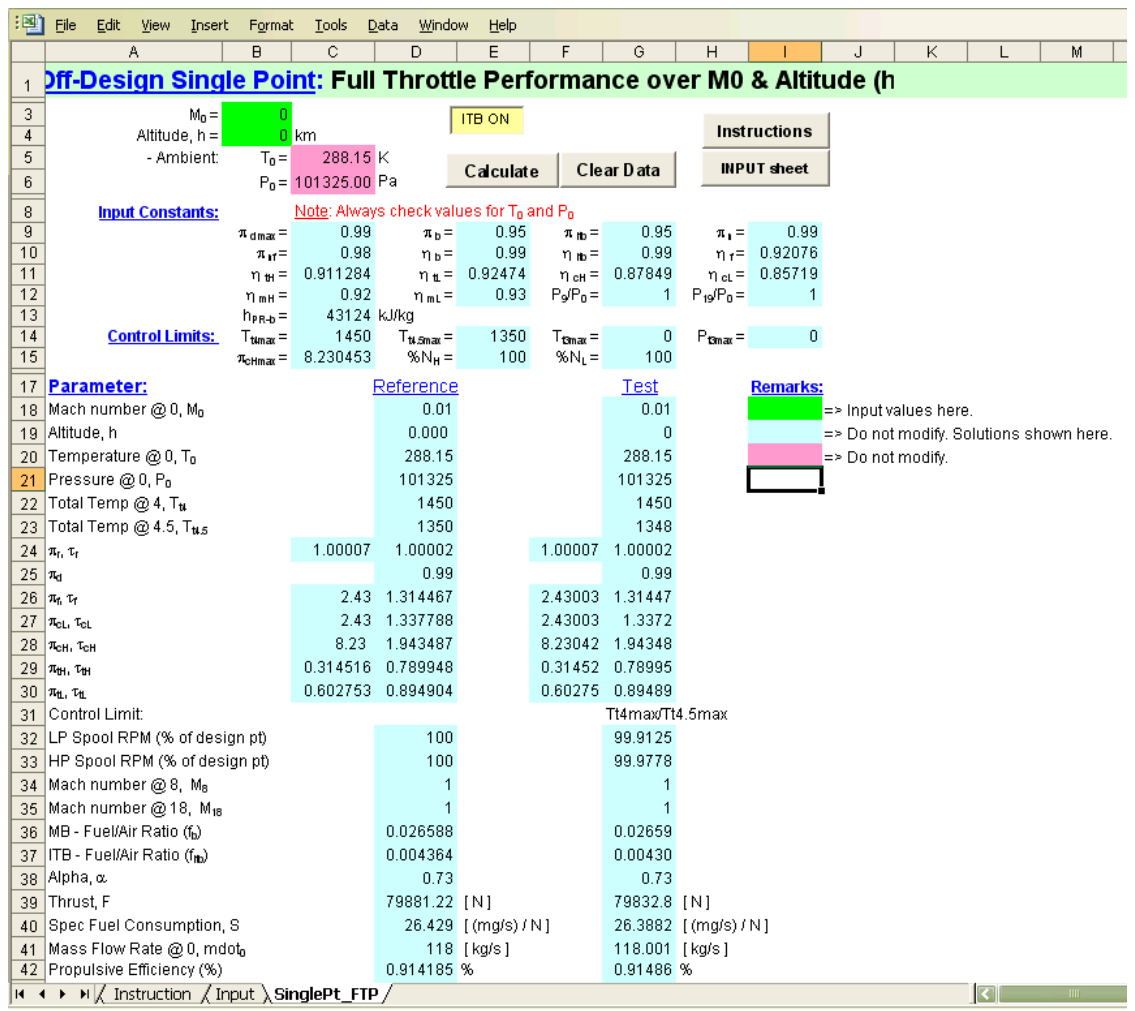

Figure H.3: Screenshot of Full-Throttle Performance: Single-point calculations

\section{H.4 Partial Throttle Performance (PTP)}

When this option is chosen, the program will open two sheets, namely, a control sheet (i.e., 'Control_PTP'), and a data sheet (i.e., 'D_PTP').

In 'Control_PTP' sheet as shown in Figure H.4, user needs to specify four input parameters, i.e., Altitude, Min \% thrust, flight parameters, and increment of temperature reduction, as indicated in 'dark green' cells.

Click the Flight parameters $\left(M_{0}\right)$ combo box to select number of plots for different $M_{0}$. Specified number of cells will be created and displayed adjacent to the Flight parameters $\left(M_{0}\right)$ combo box. Once all the 'dark green' cells are 


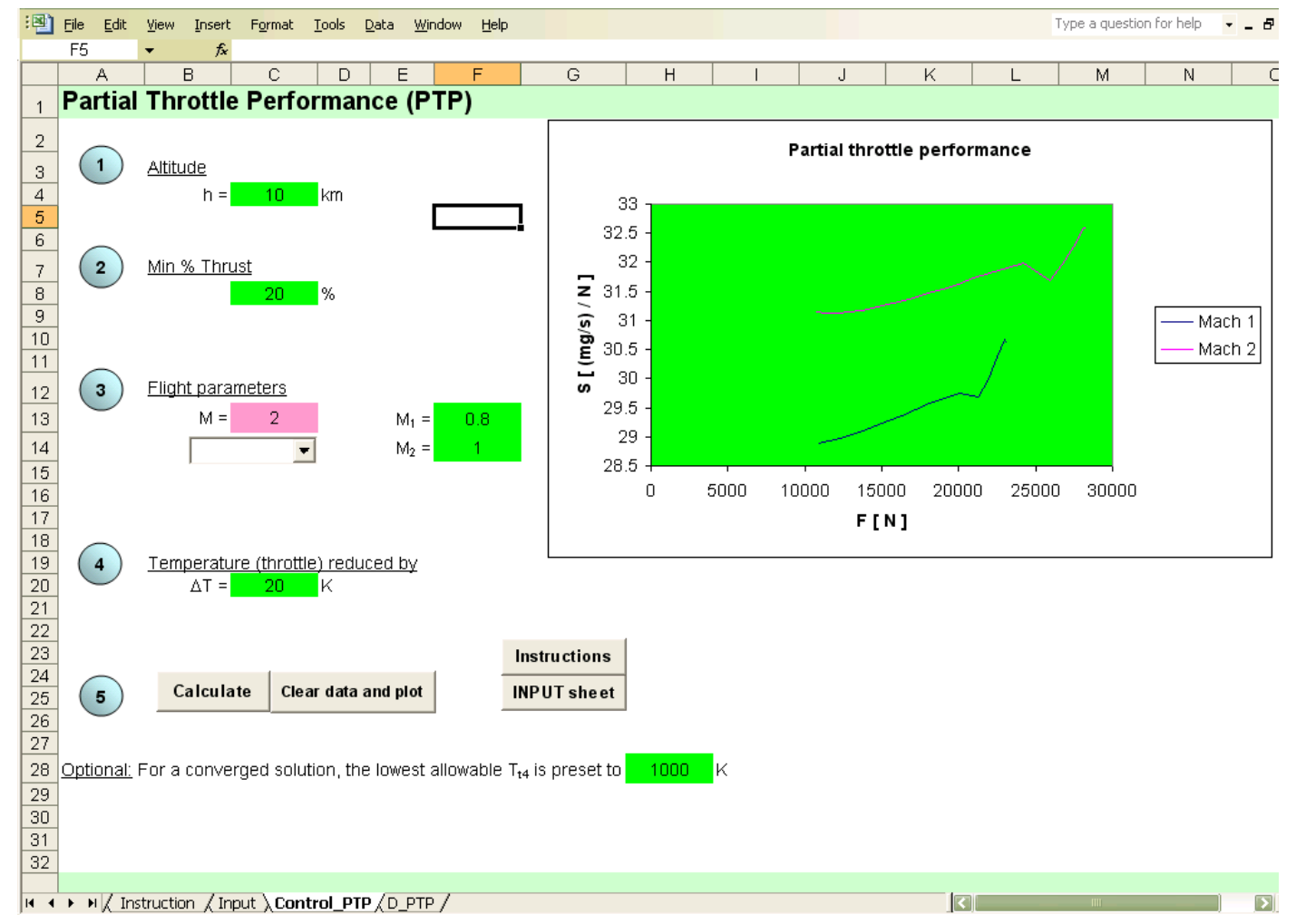

Figure H.4: Screenshot of Partial-Throttle Performance

filled, it is ready to run the program and generate the plot.

Simply pressing the Calculate button, the code will calculate the engine performance from 100\% down to minimum thrust entered in 'Min \% thrust' cell. The minimum value of 'Min \% thrust' is internally preset to be $10 \%$. If any value lower than $10 \%$ is found, user will be noticed and asked to re-enter the value.

Other than setting a Min \% thrust, user may select to enter a lowest allowable exit temperature of the main burner, $T_{t 4}$. The default value of $T_{t 4}$ is $1620^{\circ} \mathbf{R}($ or $900 \mathbf{K})$. 


\section{H.5 Discussions}

\section{H.5.1 Storing Data}

Whenever storing the output data, user can do the followings:

1. Always keep an original file of the Excel code.

2. Save the file with a new filename.

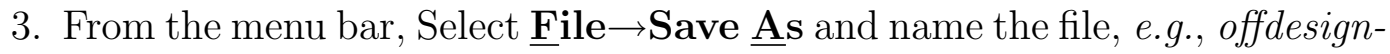
031704.xls.

4. Click Save button to confirm saving.

\section{H.5.2 Troubleshooting}

This program has been debugged several times. Therefore whenever you encounter computation errors due to either zero division or square root of a negative quantity, you will be notified through a pop up window indicating where the computation problem is. If you have any comments or bug problems you encounter in the program, you can report them to us for further improvement. Detail about the contact number can be found in the 'CoverPage' sheet of the program.

In multiple-point calculation, users may always see some of some pop-up windows telling users of the errors or warnings. There are three possibilities of having these errors or warning messages:

1. During the multiple-point computation, the design variable may be out of the range. It will happen, especially when the design variable such as flight Mach number is out of the range. For instance, the thrust of an engine is approaching zero as the flight Mach number is 5.1. If the upper limit of flight Mach number is set to be greater than 5.1 (e.g., 6), the code will not be able to proceed and thus give the warning messages. 
2. The iteration is not converging for some engine configurations at the specified flight Mach number and altitude.

3. The input values are incorrect. For instance, the main burner exit temperature $\left(T_{t 4}\right)$ may be too small for an engine to produce enough thrust. Please double check all input values and try again.

\section{H.6 Summary}

In summary, user needs to do the followings to run the program:

1. Go to the "Input" sheet.

2. Specify the Unit System (SI or English?)

3. Specify the ITB Switch. (Is the computation for engine with ITB-ON or for engine with ITB-OFF?)

4. Specify the Fuel Burn Model. (CSH or MSH model?)

5. Import Reference Conditions data from on-design code.

6. Enter all the input parameters indicated in 'dark green' cells (do not modify or change the value indicated in 'hotpink' cells).

7. Specify Perform Computation in. User will be directed to a new control sheet depending on the selection.

If you select "Full Throttle Performance (FTP): Multiple-point", do the followings in 'Control_FTP' sheet:

1. Specify a range for flight Mach number $\left(M_{0}\right)$.

2. Specify number of altitude (new 'dark green' cells will be created).

3. Specify all input parameters indicated in 'dark green'. 
4. Click Calculate button to compute and plot the results.

5. Repeat the above steps for different input parameters.

6. Go to the 'Input' sheet if you want to change some engine input parameters.

If you select "Full Throttle Performance (FTP): Single-point", do the followings in 'SinglePt_FTP' sheet:

1. Enter a value in flight Mach number $\left(M_{0}\right)$ cell.

2. Enter a value in Altitude $(h)$ cell.

3. Click Calculate button to compute and display the results.

If you select "Partial Throttle Performance (PTP)", do the followings:

1. Specify a value for altitude $(h)$.

2. Enter a value in Min \% thrust cell.

3. Specify number of flight Mach number (new 'dark green' cells will be created).

4. Specify all input parameters indicated in 'dark green'.

5. Click Calculate button to compute and plot the result.

6. Repeat the above steps for different input parameters.

7. Go to the 'Input' sheet if you want to change some engine input parameters. 


\section{Appendix I}

\section{Flowchart of Code Structure}

\section{I.1 Parametric Cycle Analysis}

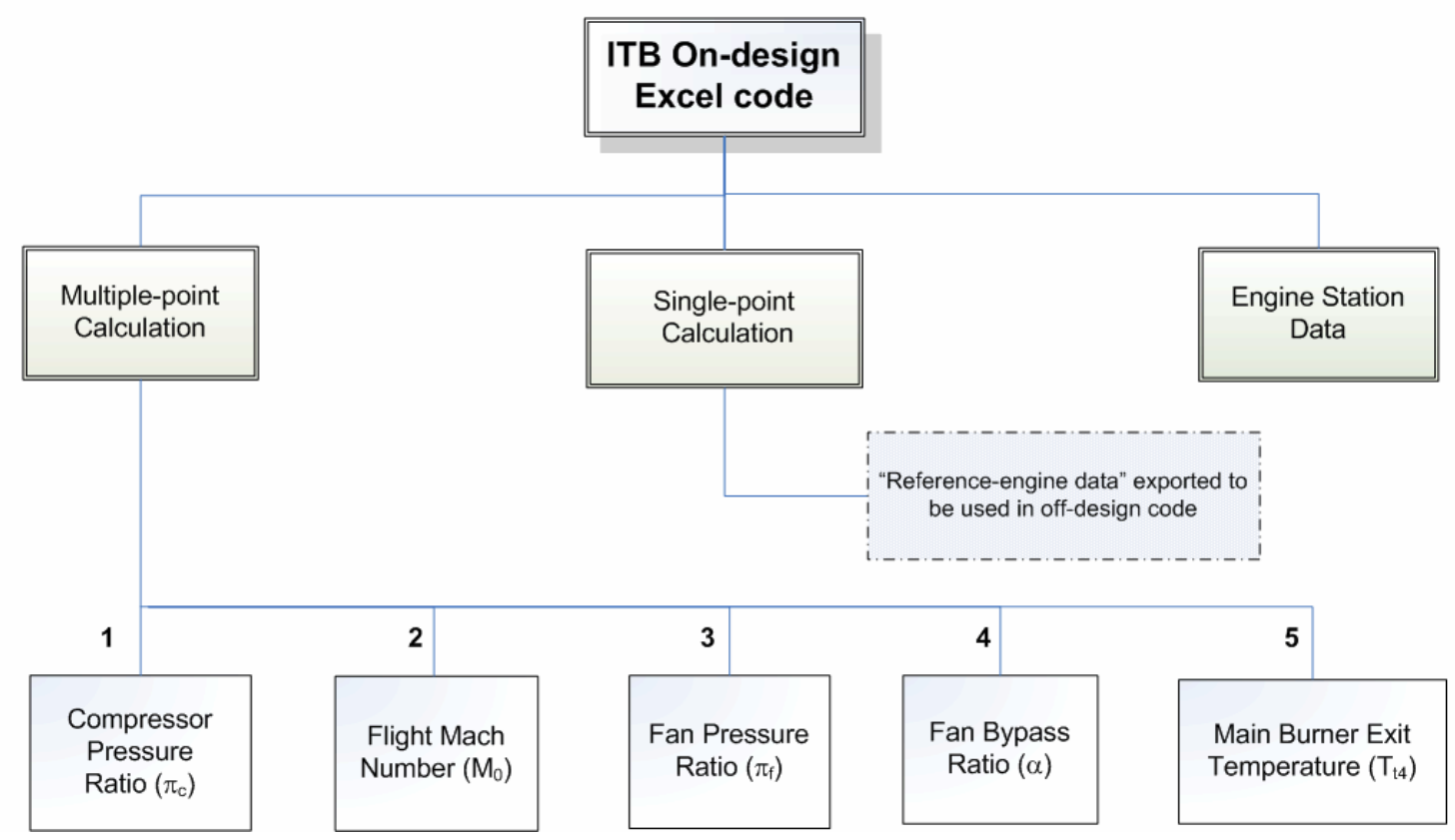

Figure I.1: Flow-chart of the main structure for ITB on-design Excel code.

[Remarks: $* D L V$ - Do Loop Variables; ${ }^{*} F L V$ - For Loop Variables.] 


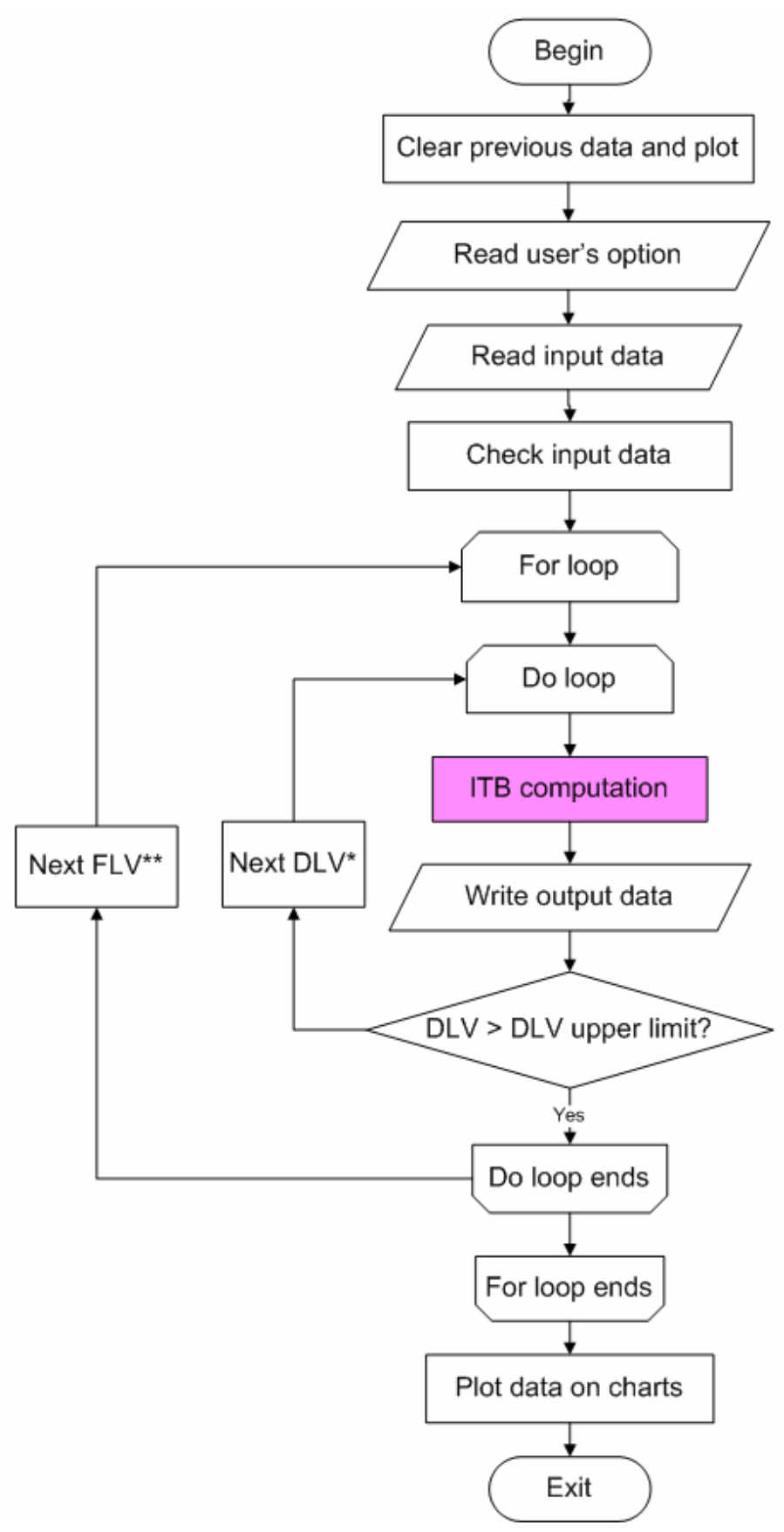

Figure I.2: Flow-chart of the iterative solution scheme for on-design multiplepoint calculation. 


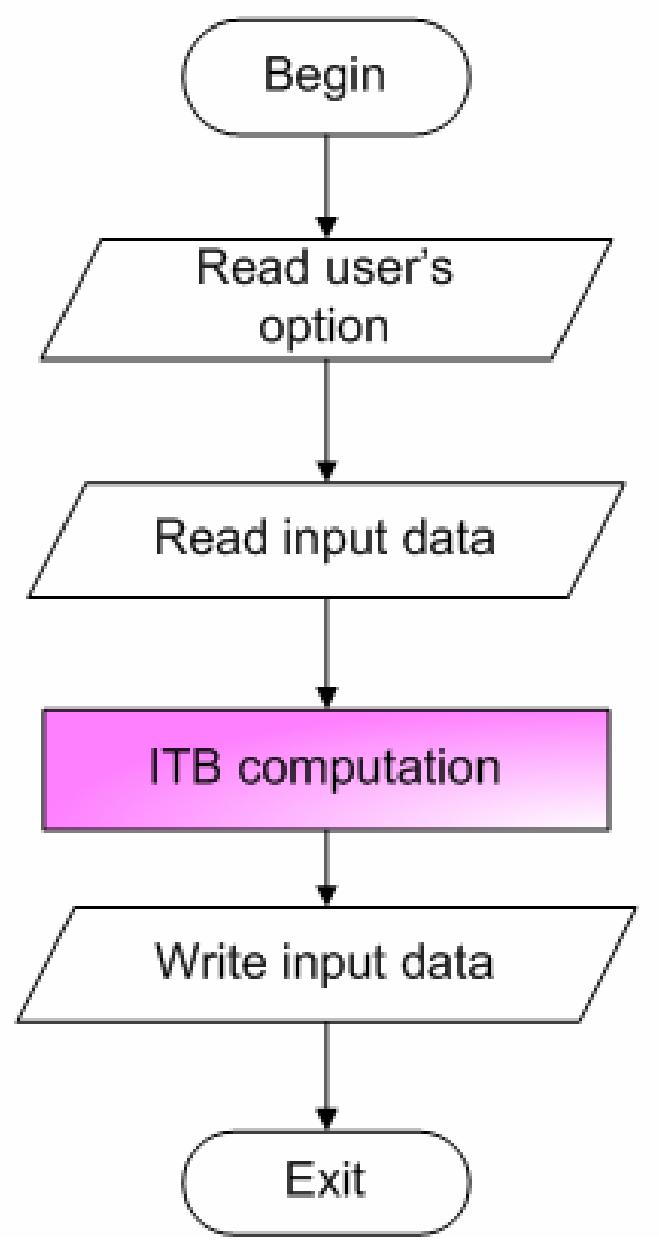

Figure I.3: Flow-chart of the iterative solution scheme for on-design single-point calculation. 


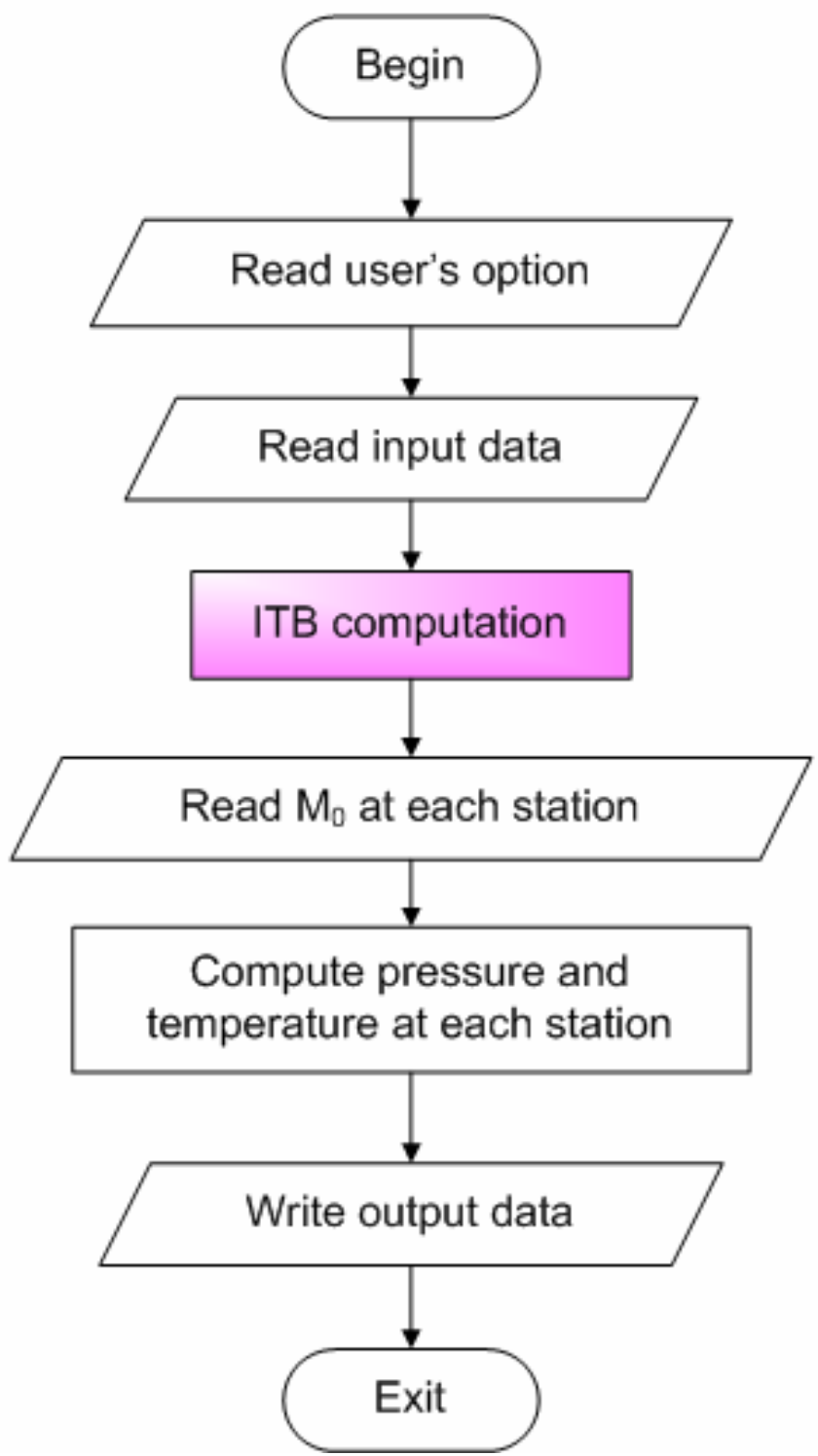

Figure I.4: Flow-chart of the solution scheme for ITB on-design single point calculation. 


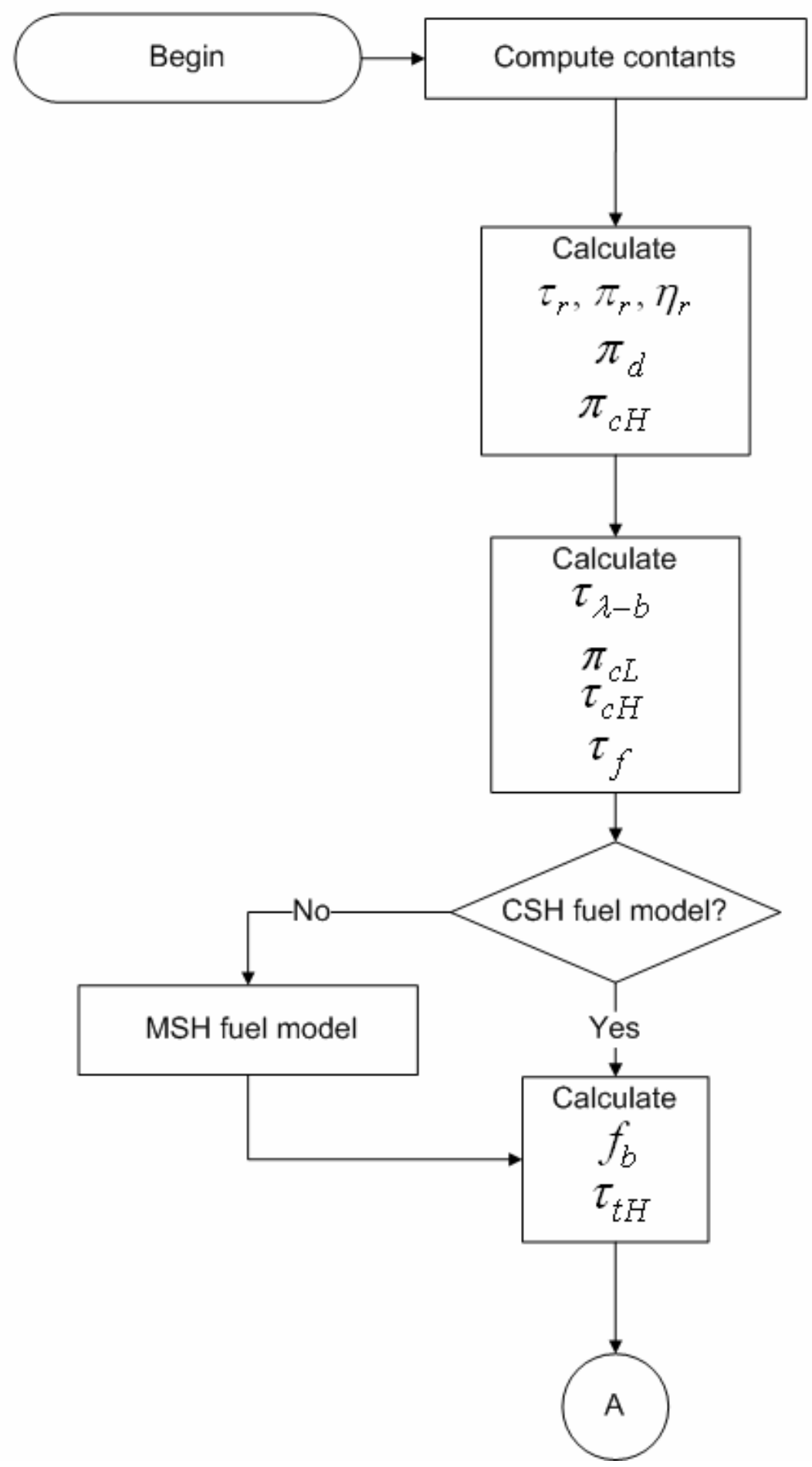

Figure I.5: Flow-chart of the solution scheme for the central ITB computation (Part 1) in on-design code. 


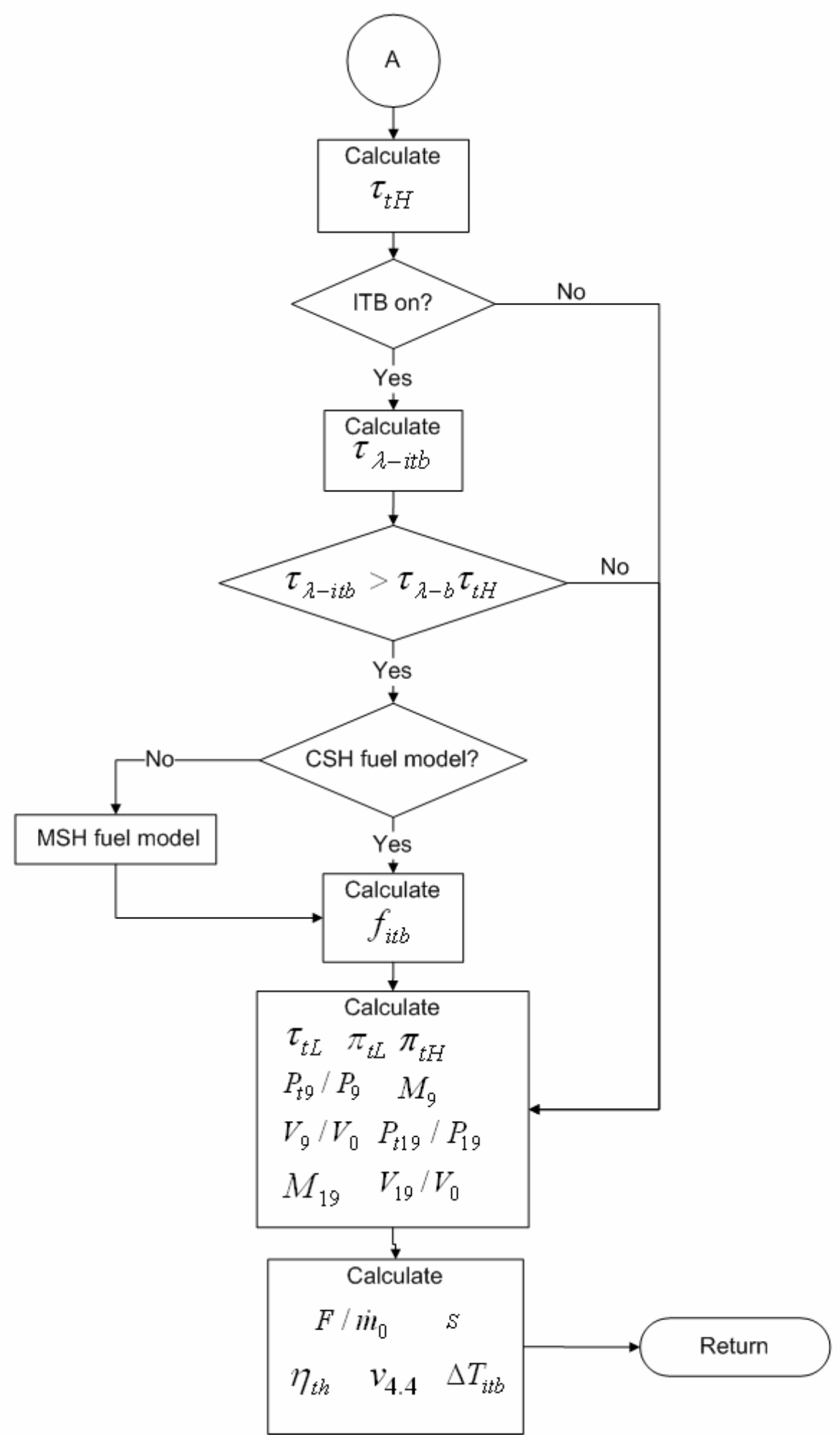

Figure I.6: Flow-chart of the solution scheme for the central ITB computation (Part 2) in on-design code. 


\section{I.2 Performance Cycle Analysis}

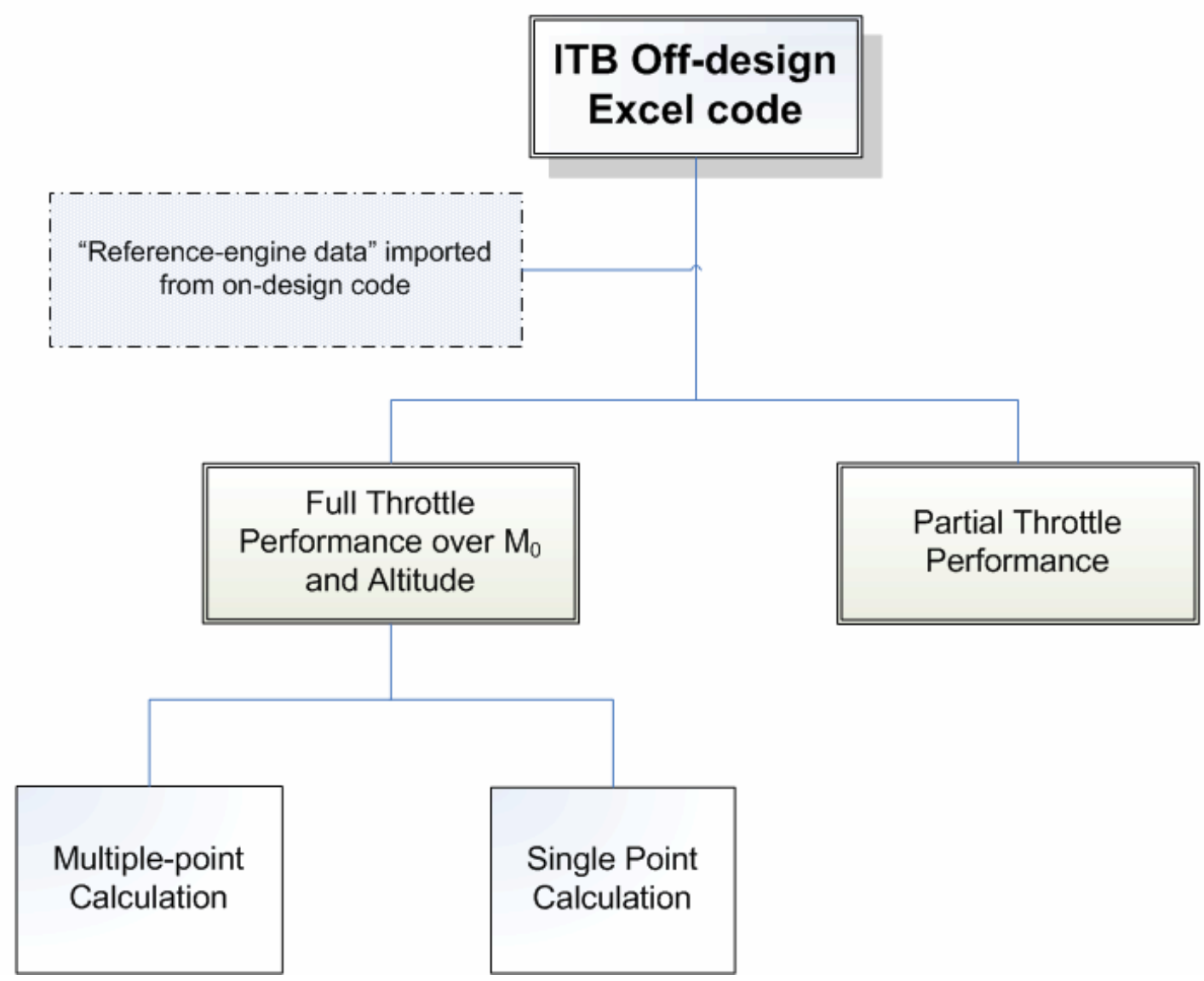

Figure I.7: Flow-chart of the main structure for ITB off-design Excel code. 


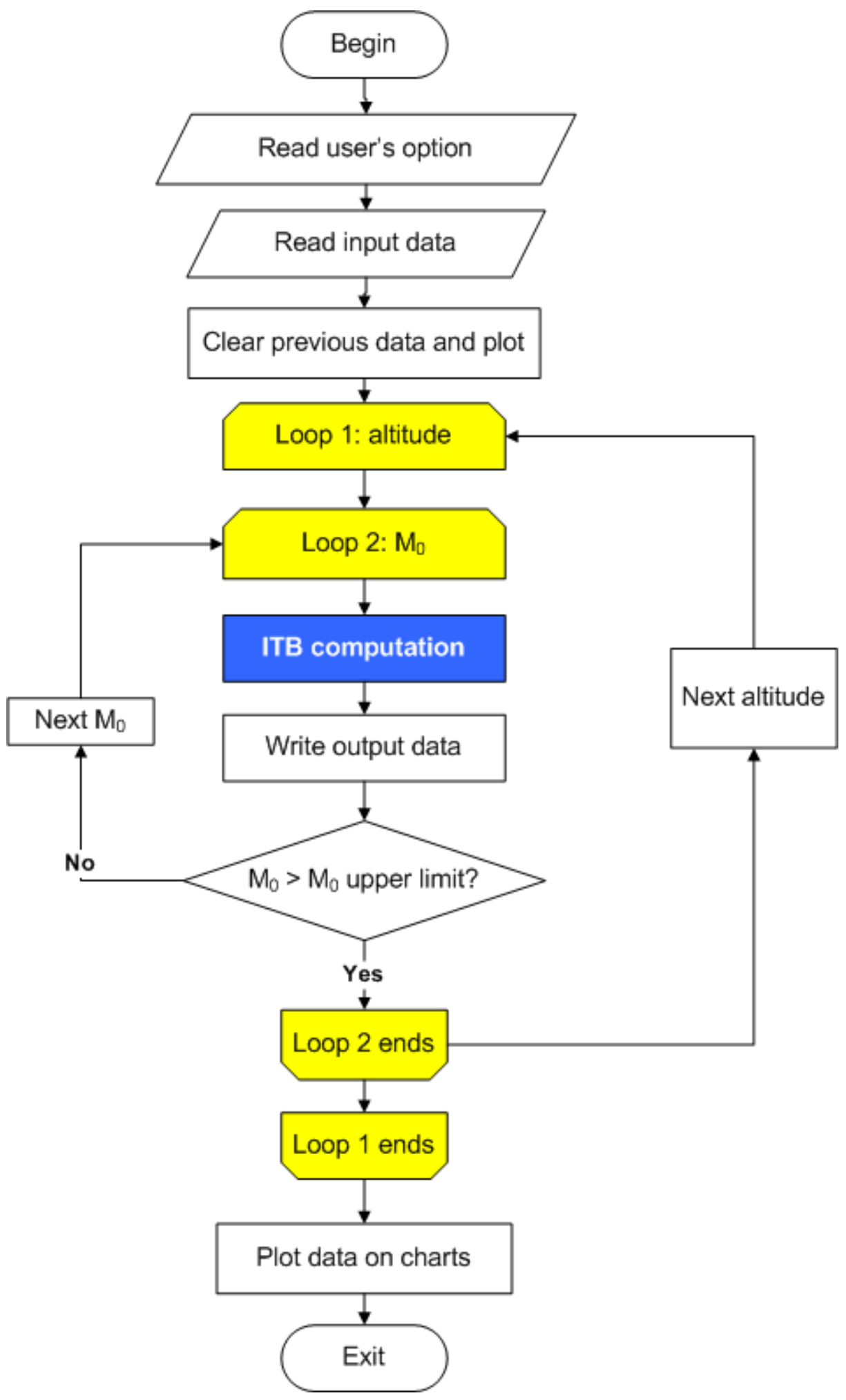

Figure I.8: Flow-chart of the iterative solution scheme for FTP in off-design code. 


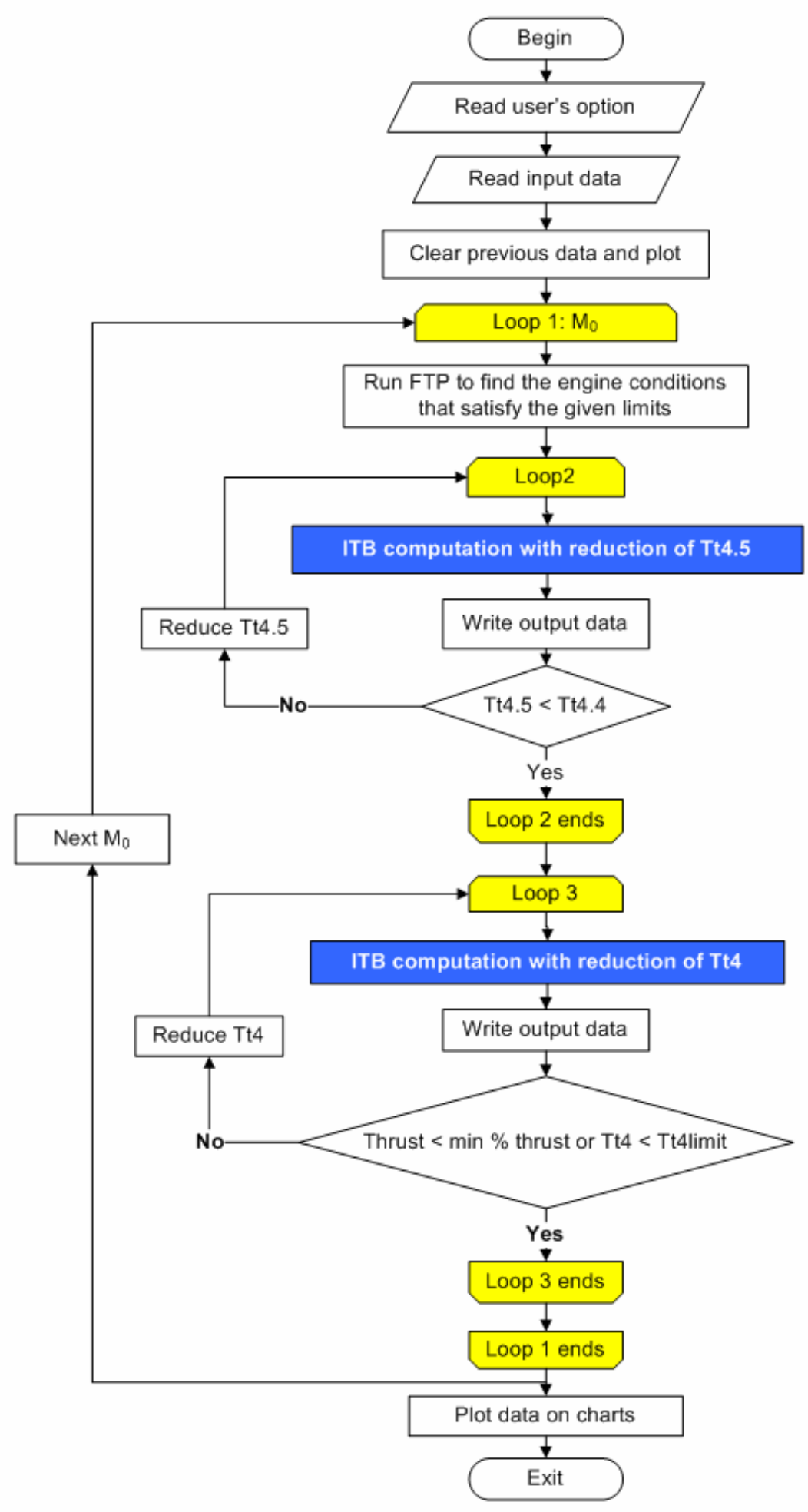

Figure I.9: Flow-chart of the iterative solution scheme for PTP in off-design code. 


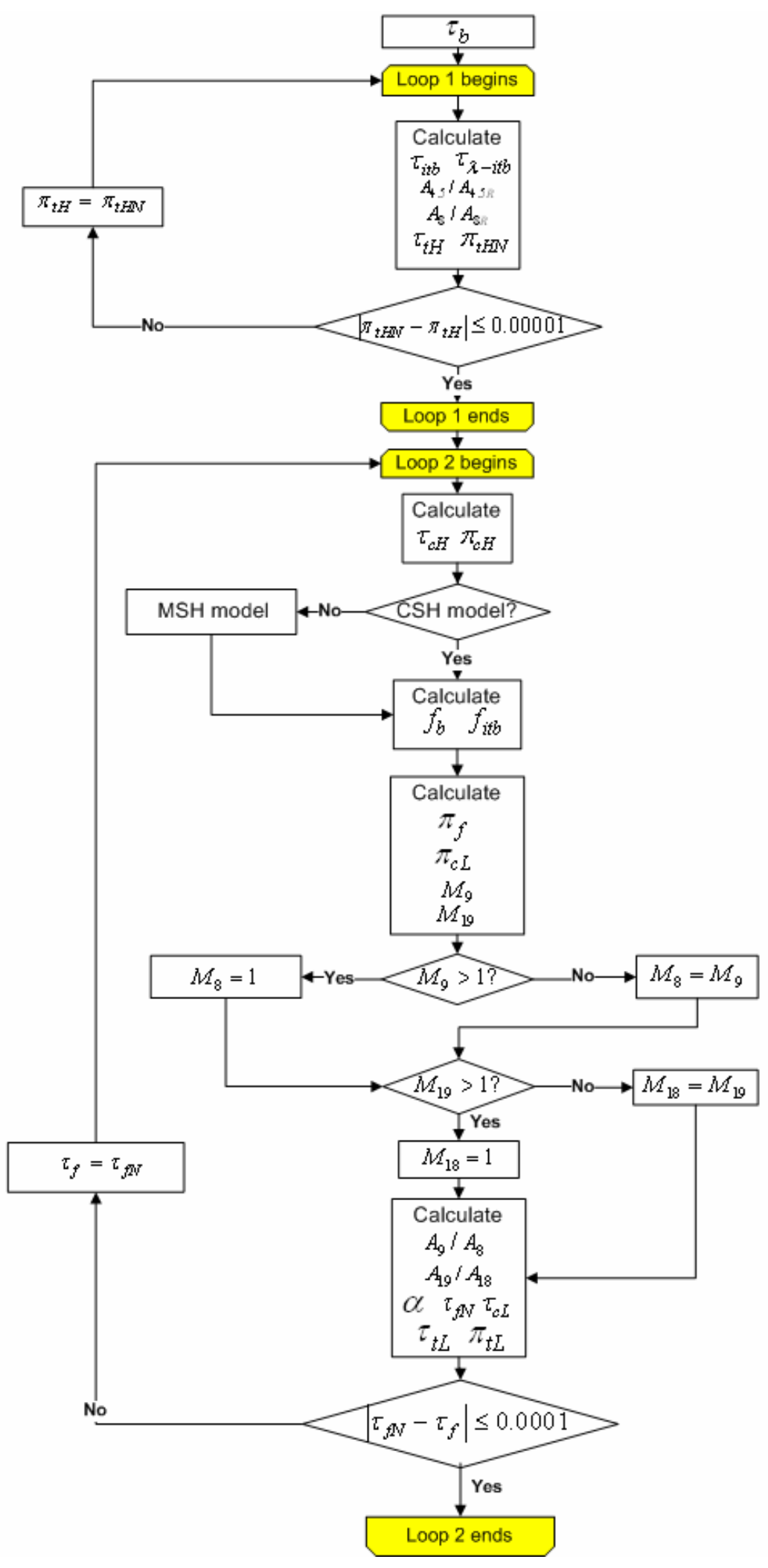

Figure I.10: Flow-chart of the iterative solution scheme for the subroutine itbperf2 (i.e., central ITB computation) in off-design code. 


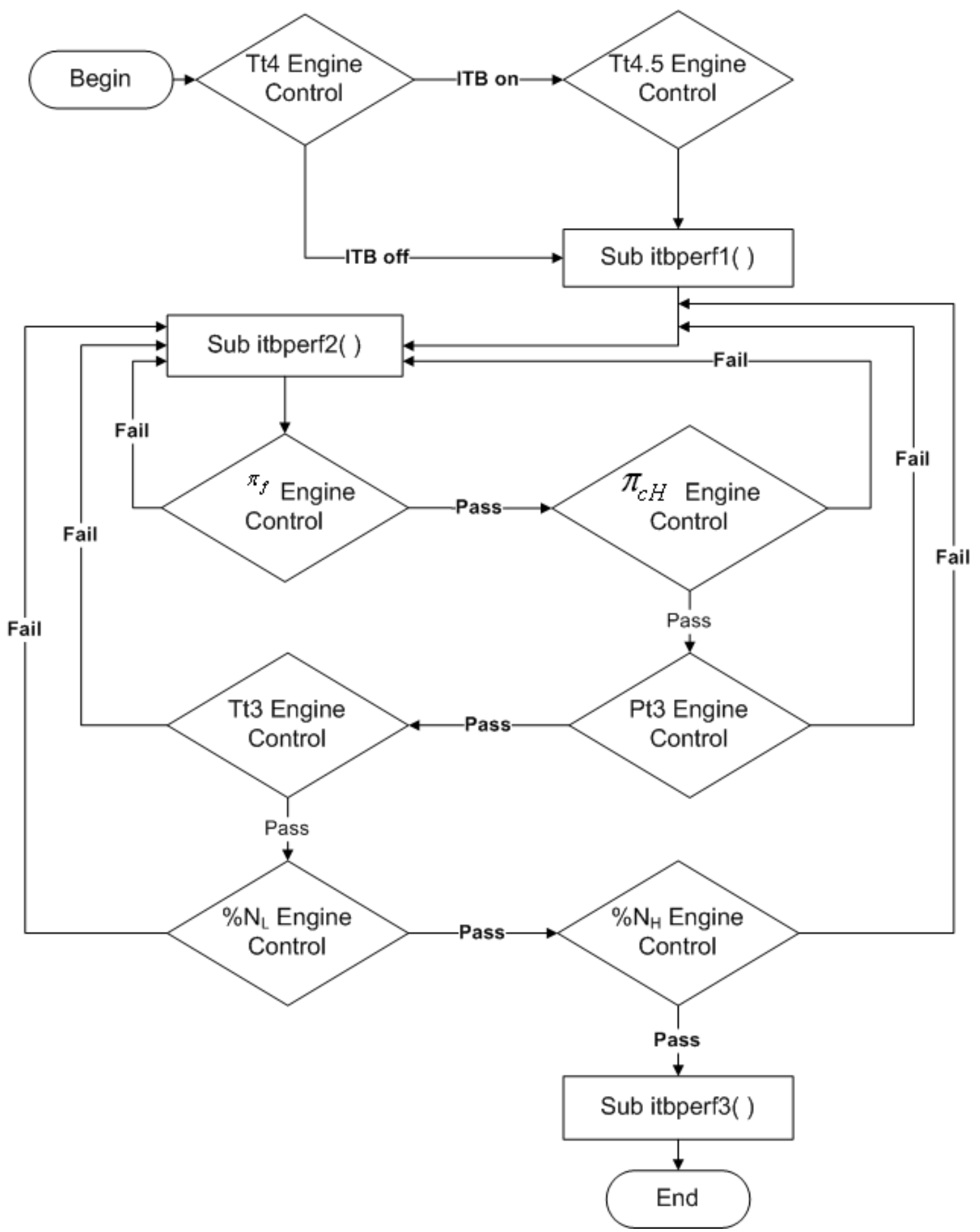

Figure I.11: Flow-chart of the engine control limits in off-design code. 


\section{Appendix $\mathbf{J}$}

\section{Summary of Input Data for Each Computation}

Table J.1: Turbofan engine configuration in the parametric cycle analysis.

\begin{tabular}{|c|c|}
\hline Description & Input value \\
\hline \multicolumn{2}{|c|}{ Polytropic efficiencies } \\
\hline Fan $e_{f}$ & 0.93 \\
\hline $\mathrm{HPC} e_{c H}$ & 0.9085 \\
\hline $\mathrm{HPT} e_{t H}$ & 0.8999 \\
\hline $\mathrm{LPC} e_{c L}$ & 0.8738 \\
\hline $\mathrm{LPT} e_{t L}$ & 0.9204 \\
\hline \multicolumn{2}{|c|}{ Total pressure ratios } \\
\hline Inlet $\pi_{d \max }$ & 0.99 \\
\hline Main burner $\pi_{b}$ & 0.96 \\
\hline ITB $\pi_{i t b}$ & 0.96 \\
\hline Nozzle $\pi_{n}$ & 0.99 \\
\hline Fan nozzle $\pi_{n f}$ & 0.98 \\
\hline \multicolumn{2}{|c|}{ Component efficiencies } \\
\hline Main burner $\eta_{b}$ & 0.98 \\
\hline ITB $\eta_{i t b}$ & 0.98 \\
\hline High-pressure spool $\eta_{m H}$ & 0.92 \\
\hline Low-pressure spool $\eta_{m L}$ & 0.93 \\
\hline Fuel low heating value $h_{P R}$ & $42798.4 \mathrm{~kJ} / \mathrm{kg}$ \\
\hline Bleed air fraction $\beta$ & $1 \%$ \\
\hline \multicolumn{2}{|l|}{ Options } \\
\hline Turbine cooling & $\mathrm{ON}$ \\
\hline Fuel model & $\mathrm{CSH}$ \\
\hline
\end{tabular}


Table J.2: Input data for the result comparison between the Excel codes and AEDsys suite in Chapter 4 and 5 .

\begin{tabular}{|c|c|}
\hline Design variable & $M_{0}$ \\
\hline Regime & high FBR \\
\hline Flight Condition & \\
$M_{0}$ & 0.9 \\
$\mathrm{~h}(\mathrm{~km})$ & 10 \\
\hline Operating Conditions & \\
$\pi_{c L}$ & 2.0 \\
$\pi_{c}$ & 28.48 \\
$\pi_{f}$ & 2.0 \\
$T_{t 4}(\mathrm{~K})$ & 1500 \\
$\alpha$ & 4.0 \\
$\dot{m}_{0}(\mathrm{~kg} / \mathrm{s})$ & 118 \\
\hline Options & \\
Nozzle & Convergent \\
Turbine cooling & ON \\
Fuel model & CSH \\
ITB & OFF \\
\hline \multicolumn{2}{|c}{} \\
\hline
\end{tabular}


Table J.3: Input data for the parametric cycle analysis in Chapter 6(Part 1).

\begin{tabular}{|c|c|c|c|}
\hline Design variable & $M_{0}$ & $M_{0}$ & \multirow{2}{*}{ Turbine Cooling } \\
\hline Regime & low FBR & high FBR & \\
\cline { 1 - 3 } Flight Condition & & & 10 \\
$\mathrm{~h}(\mathrm{~km})$ & 10 & 10 & \\
Operating Conditions & & & 1.387 \\
$\pi_{c L}$ & 1.387 & 1.387 & 28.48 \\
$\pi_{c}$ & 28.48 & 28.48 & 1.3 \\
$\pi_{f}$ & 2.5 & 1.3 & 1800 \\
$T_{t 4}(\mathrm{~K})$ & 1600 & 1600 & 1300 \\
$T_{t 4.5}$ & 1300 & 1300 & 4.0 \\
$\alpha$ & 0.5 & 4.0 & Convergent \\
Nozzle & $\mathrm{CD}$ & Convergent & \\
\hline
\end{tabular}

Table J.4: Input data for the parametric cycle analysis in Chapter 6 (Part 2).

\begin{tabular}{|c|c|c|c|c|}
\hline Design variable & $T_{t 4}$ & $T_{t 4}$ & CPR & CPR \\
\hline Regime & subsonic & supersonic & subsonic & supersonic \\
\hline Flight Condition & & & & \\
$M_{0}$ & 0.85 & 1.2 & 0.85 & 1.2 \\
$\mathrm{~h}(\mathrm{~km})$ & 10 & 10 & 10 & 10 \\
\hline Operating Conditions & & & & \\
$\pi_{c L}$ & 1.387 & 1.387 & 1.387 & 1.387 \\
$\pi_{c}$ & 28.48 & 28.48 & - & - \\
$\pi_{f}$ & 1.3 & 3.0 & 1.3 & 3.0 \\
$T_{t 4}(\mathrm{~K})$ & - & - & 1600 & 1600 \\
$T_{t 4.5}$ & 1300 & 1300 & 1300 & 1300 \\
$\alpha$ & 4.0 & 0.5 & 4.0 & 0.5 \\
Nozzle & $\mathrm{CD}$ & Convergent & $\mathrm{CD}$ & Convergent \\
\hline
\end{tabular}


Table J.5: Input data for the parametric cycle analysis in Chapter 6 (Part 3).

\begin{tabular}{|c|c|c|c|c|}
\hline Design variable & FPR & FPR & FBR & FBR \\
\hline Regime & subsonic & supersonic & subsonic & supersonic \\
\hline Flight Condition & & & & \\
$M_{0}$ & 0.85 & 1.2 & 0.85 & 1.2 \\
$\mathrm{~h}(\mathrm{~km})$ & 10 & 10 & 10 & 10 \\
\hline Operating Conditions & & & & \\
$\pi_{c L}$ & 1.387 & 1.387 & 1.387 & 1.387 \\
$\pi_{c}$ & 28.48 & 28.48 & - & - \\
$\pi_{f}$ & - & - & 1.3 & 3.0 \\
$T_{t 4}(\mathrm{~K})$ & 1600 & 1600 & 1600 & 1600 \\
$T_{t 4.5}$ & 1300 & 1300 & 1300 & 1300 \\
$\alpha$ & 4.0 & 0.5 & - & - \\
Nozzle & $\mathrm{CD}$ & Convergent & $\mathrm{CD}$ & Convergent \\
\hline
\end{tabular}

Table J.6: Hidden data on 'Input' sheet of the off-design cycle analysis Excel code.

\begin{tabular}{|c|c|c|c|c|c|}
\hline \multirow{3}{*}{$\begin{array}{l}\text { Column } \rightarrow \\
\text { Row } \downarrow 26\end{array}$} & \multicolumn{3}{|c|}{$\begin{array}{l}\text { ITB OFF } \\
\text { condition }\end{array}$} & \multirow{2}{*}{\begin{tabular}{|c|}
$\begin{array}{c}\text { Single-point } \\
\text { ITB ON/OFF }\end{array}$ \\
16
\end{tabular}} & \multirow{2}{*}{$\begin{array}{c}\begin{array}{r}\text { ITB ON } \\
\text { condition }\end{array} \\
17\end{array}$} \\
\hline & 13 & 14 & 15 & & \\
\hline & Thrust & $\pi_{t L}$ & $\mathrm{ON} / \mathrm{OFF}$ & $\eta_{t h}$ & $f_{i t b}$ \\
\hline 27 & $\mathrm{~S}$ & $\tau_{t L}$ & $\epsilon_{1}$ & $\eta_{p}$ & $\pi_{i t b}$ \\
\hline 28 & $\eta_{t h}$ & $\eta_{t L}$ & $\epsilon_{2}$ & $\eta_{o}$ & \\
\hline 29 & $\eta_{p}$ & $f_{i t b}$ & & & \\
\hline 30 & $\eta_{o}$ & $\pi_{i t b}$ & & & \\
\hline 31 & & $M_{8}$ & & & \\
\hline
\end{tabular}




\section{Appendix K}

\section{Other Contribution: Tracking a Single Evaporating Drop in a Crossflow}

Besides developed the cycle analysis codes, the author also developed an Excel code for tracking a single evaporating drop in a crossflow. The details of the Excel code and its user guide can be found in [27]. The following sections a general approach to model the behavior of the drop in a crossflow and the numerical method for the computation.

\section{K.1 Introduction}

A liquid spray injected into a gaseous crossflow with high temperature is important because of its wide application in systems involving two phase mixing. It is therefore important to be able to compute this flow to optimize the mixing strategy.

An existing Excel program [52] has previously been developed for tracking a single droplet in crossflow computation. This work is focused on producing a quick computational method for determining spray penetration with evaporation. 
With this spreadsheet, one can investigate the dispersion of an air0blast atomized spray jet into a high temperature crossflow. During the transverse injection of a spray into high velocity flow, the droplets (carried along in the gaseous stream of co-flowing air) are not only subjected to forces due to the crossflow motion, but also to increases in the combustor temperature and pressure (Figure K.1.).

\section{K.2 Droplet Trajectories and Velocities}

The trajectories of the droplets can be tracked by applying a Langrangianbased analysis to the droplets. The momentum equations for a droplet can be obtained by equating the droplet motion to:

1. the viscosity and pressure-related drag forces.

2. the pressure gradient and viscous forces related to the fluid surrounding the droplet.

3. the inertia of the virtual mass, which is induced when the particle acceleration affects the fluid mass acceleration.

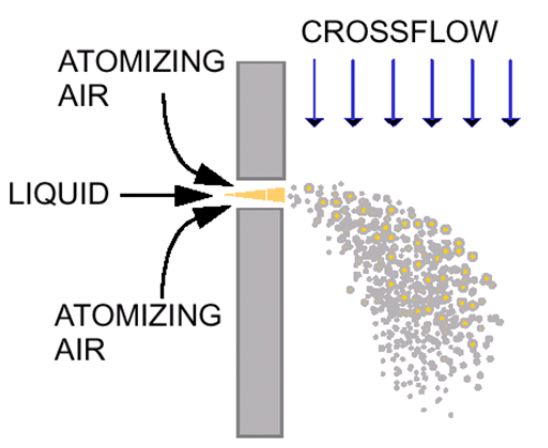

Figure K.1: Airblast-Atomized Fuel Spray Injected Into a Crossflow of Air [23]

Droplet trajectory and velocity with respect to time can be calculated based on these principles along with the following assumptions:

1. The droplet is spherical. 
2. No droplet breakup occurs.

3. Vaporization is not considered yet and will be presented in Section K.3.

4. Lift, virtual mass, and Basset forces which takes into account the acceleration history of the droplet, are neglected.

5. Chemical reaction is not included.

These assumptions reduce the droplet momentum equation to include only the effects of the drag and body forces. The general momentum equations for a single droplet injected along the positive $\mathrm{x}$-direction, transversely into a downward-flowing air stream in the positive z-direction, as shown in Figure K.2. is described by

$$
\vec{F}_{d}=\vec{F}_{d r a g}+\vec{F}_{b o d y}
$$

where the net force $\vec{F}_{d}$ that drives the droplet motion is balanced by the drag force opposing its motion, and the field forces acting on the droplet. The aerodynamic drag force is given by

$$
\vec{F}_{\text {drag }}=-\frac{1}{2} \rho_{A} \vec{U}_{R}\left|\vec{U}_{R}\right| A_{d} C_{D}
$$

where $\rho_{A}$ is the air density, and $A_{d}$ and $C_{D}$, the projected area and the drag coefficient of the droplet, respectively. The relative velocity between the droplet and the crossflow has a magnitude of $U_{R}$ (Figure K.2).

The body force, resulting from an equivalent volume of air that buoys the droplet, includes the gravitational and buoyancy forces. It is given by

$$
\vec{F}_{b o d y}=\left(\rho_{d}-\rho_{A}\right) V_{d} \overrightarrow{g_{0}}
$$




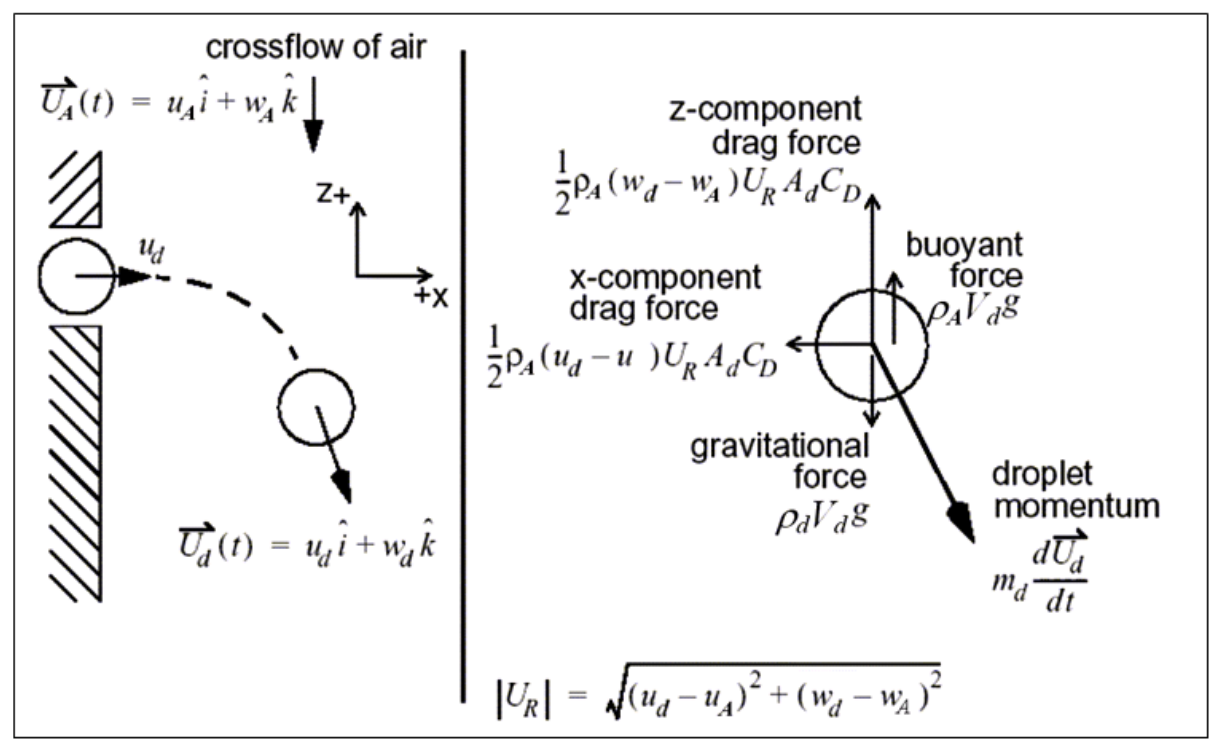

Figure K.2: The Free-body Diagram of a Single Droplet in Crossflow [23]

which says that the body force is equal to the product of relative droplet and air density $\left(\rho_{d}-\rho_{A}\right)$, the droplet volume $V_{d}$, and gravitational acceleration $\overrightarrow{g_{0}}$.

Substituting equation K.2 and equation K.3 to equation K.1 yields:

$$
\begin{gathered}
\rho_{d} V_{d} \frac{d u_{d}}{d t}=-\frac{1}{2} \rho_{A}\left(u_{d}-u_{A}\right)\left|\vec{U}_{R}\right| A_{d} C_{D} \\
\rho_{d} V_{d} \frac{d w_{d}}{d t}=-\frac{1}{2} \rho_{A}\left(w_{d}-w_{A}\right)\left|\vec{U}_{R}\right| A_{d} C_{D}+\left(\rho_{A}-\rho_{d}\right) V_{d} g_{0} \\
\frac{d x}{d t}=u_{d} \\
\frac{d z}{d t}=w_{d}
\end{gathered}
$$

The drag coefficient of the droplet depends on the droplet Reynolds number and is given by 


$$
C_{D}= \begin{cases}\frac{24}{R e_{d}}\left[1+\frac{1}{6} R e_{d}^{2 / 3}\right] & \text { for } R e_{d} \leq 1000 \\ 0.424 & \text { for } R e_{d}>1000\end{cases}
$$

where $R e_{d}$ is the droplet Reynolds number and is defined as follows

$$
R e_{d}=\frac{2 \rho_{A}\left|\vec{U}_{R}\right| r_{d}}{\mu_{A}}
$$

in which $r_{d}$ is the droplet radius and $\mu_{A}$ is the gas (air) viscosity.

\section{K.3 Droplet Evaporation}

To include the effect of evaporation rate on spray penetration, apply a control volume at droplet surface that will change with droplet radius during evaporation process. For simplicity, consider steady state analysis first.

\section{K.3.1 Steady-state Analysis}

A fuel droplet rarely reaches a steady state during its lifetime [5]. This is because most commercial fuels are multi-component, where different fuel compounds posses its own properties, for example kerosene and gasoline. To simplify analysis, 'steady state' term here refers to 'quasi-steady', which allows droplet lifetime and evaporation rate to be estimated to an acceptable level of accuracy.

To simplify the analysis, in addition to the assumptions listed in trajectory analysis as shown in Section K.2.

1. There is no radiation.

2. There is no internal circulation and internal convective heating within droplet. 
3. Consider only single-component fuel (with well-defined boiling point).

4. It is quasi-steady flow.

Consider a fuel droplet at low fuel injection temperature that is suddenly exposed to a gaseous crossflow at high temperature. Initially, almost all heat supplied to the droplet serves to raise the droplet temperature. As the droplet temperature rises, fuel vapor will form at the droplet surface and has two main effects:

1. A large portion of heat transferred to droplet is used to vaporize the droplet.

2. The outward flow of fuel vapor impedes the rate of heat transfer to droplet.

Eventually, a stage is reached where all heat transferred to droplet is used as the heat of vaporization and the droplet temperature will stabilize at a steadystate temperature.

\section{Mass Transfer Number}

Assume that the thermal diffusion is negligible. Therefore, the concentration gradient is the only driving force considered for species diffusion in the direction of the diffusion path. Then, the following expression for an evaporating fuel droplet of radius $\mathrm{r}$ is described by:

$$
\frac{d Y_{F}}{d r}=-\frac{R T}{D_{F A} P}\left(\dot{m}_{F}^{\prime \prime} Y_{A}\right)
$$

where $D_{F A}$ is the diffusion coefficient of fuel in air

$\dot{m}_{F}^{\prime \prime}$ is the mass rate of diffusion per unit area (mass flux)

$P \quad$ is the ambient air pressure

$R \quad$ is the universal gas constant

$T$ is the ambient air temperature

$Y_{F}(r)$ is the fuel mass fraction

$Y_{A} \quad$ is the air mass fraction at range $r_{s}<r<\infty$ at any time 


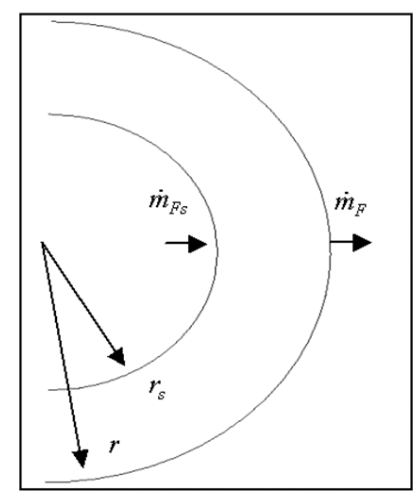

Figure K.3: Control Surface Surronding a Single Evaporating Droplet

$$
Y_{A}=1-Y_{F}
$$

From the continuity equation applied on the control surface surrounding a droplet (Figure K.3), one obtains

$$
\dot{m}_{F}^{\prime \prime}=\dot{m}_{F, s}^{\prime \prime}\left(\frac{r_{s}}{r}\right)^{2}
$$

where $\dot{m}_{F, s}^{\prime \prime}$ is the mass flux at droplet surface

$r_{s} \quad$ is the radius of droplet

$r \quad$ is the radius of control surface at time $t$

Substituting Eqs. (K.11) and (K.12) into (K.10) yields

$$
\frac{d Y_{F}}{d r}=-\frac{R T}{D_{F A} P} \dot{m}_{F}^{\prime \prime}\left(\frac{r_{s}}{r}\right)^{2}\left(1-Y_{F, s}\right)
$$

where $Y_{F, s}$ is the fuel mass fraction at droplet surface.

Assume the ideal gas relation $\left(\rho=\frac{P}{R T}\right)$, separating variables, integrating, and rearranging Eq. K.13 yields

$$
\dot{m}_{F, s}^{\prime \prime}=-\frac{\rho D_{F A}}{r_{s}} \ln \left(1-Y_{F, s}\right)
$$


where $\rho$ is the fuel density.

Multiplying by droplet surface area $\left(A_{s}=4 \pi r_{s}\right)$ and for $D=2 r_{s}$,

$$
\dot{m}_{F, s}^{\prime \prime}=-2 \pi D \rho D_{F A} \ln \left(1-Y_{F, s}\right)
$$

where $D$ is diameter of evaporating droplet.

\section{Unity Lewis Number (Le)}

Assume $L e=1$, it implies that the mass transfer rate is equal to the heat transfer rate, i.e.,

$$
D_{F A}=\alpha_{A}
$$

where

$$
\alpha_{A}=\left(\frac{k}{\rho c_{p}}\right)_{A}
$$

where $\alpha_{A}$ is the thermal diffusivity of air

$k$ is the thermal conductivity

$c_{p}$ is the specific heat at constant pressure

Then, Eq. (K.16) becomes

$$
\rho_{A} D_{F A}=\left(\frac{k}{\rho c_{p}}\right)_{A}
$$


Define the mass transfer number, $B_{M}$ :

$$
B_{M}=\frac{Y_{F, s}-Y_{F, \infty}}{Y_{F, d r o p}-Y_{F, s}}
$$

Since $Y_{F, \infty} \approx 0$ and $Y_{F, \text { drop }}=1$, Eq. K.19) is simplified as

$$
B_{M}=\frac{Y_{F, s}}{1-Y_{F, s}}
$$

where

$$
Y_{F, s}=\left[1+\left(\frac{P}{P_{F, s}}\right)\left(\frac{M_{A}}{M_{F}}\right)\right]
$$

where $\quad M_{F}$ is the molecular weight of fuel $[\mathrm{kg} / \mathrm{kg}-\mathrm{mol}]$

$M_{A}$ is the molecular weight of fuel $[\mathrm{kg} / \mathrm{kg}-\mathrm{mol}]$

$P \quad$ is the ambient pressure $[k P a]$

$P_{F, s}$ is the fuel vapor pressure at droplet surface $[k P a]$

Rearranging $\ln \left(1-Y_{F}\right)$ in term of $B_{M}$ yields

$$
\ln \left(1-Y_{F}\right)=-\ln \left(1+B_{M}\right)
$$

Substituting Eqs. (K.18) and (K.22) into (K.15) yields the rate of evaporation of a fuel drop at the surface:

$$
\dot{m}_{F, s}^{\prime \prime}=2 \pi D\left(\frac{k}{c_{p}}\right)_{A} \ln \left(1+B_{M}\right)
$$

\section{Reference Condition}

For better accuracy, the choice of values of $k_{g}$ and $c_{p, g}$ are evaluated at the following reference temperature $\left(T_{r}\right)$ and composition $\left(Y_{r}\right)$ using the "one-third 
rule":

$$
\begin{gathered}
T_{r}=T_{s}+\frac{1}{3}\left(T_{\infty}-T_{s}\right) \\
Y_{F, r}=Y_{F, s}+\frac{1}{3}\left(Y_{F, \infty}-Y_{F, s}\right)
\end{gathered}
$$

Since $Y_{F, s} \approx 0$

$$
\begin{gathered}
Y_{F, r}=\frac{2}{3} Y_{F, s} \\
Y_{A, r}=1-Y_{F, r}
\end{gathered}
$$

\section{Fuel-air mixture}

Therefore, the reference thermal conductivity and specific heat at constant pressure are estimated as

$$
\begin{gathered}
k_{g}=Y_{A, r} \cdot k_{A}\left(T_{r}\right)+Y_{F, r} \cdot k_{F v}\left(T_{r}\right) \\
c_{p, g}=Y_{A, r} \cdot c_{p, A}\left(T_{r}\right)+Y_{F, r} \cdot c_{p, F v}\left(T_{r}\right)
\end{gathered}
$$

\section{Evaporation Constant}

At steady-state period, the droplet diameter $D$ at any instant may be related to its initial diameter $D_{0}$ by $D^{2}$-law:

$$
D_{0}^{2}-D^{2}=\lambda_{s t} t
$$

where $\lambda_{s t}\left[\frac{m^{2}}{s}\right]$ is the steady state evaporation constant 


$$
\lambda_{s t}=\left(\frac{k}{c_{p}}\right) \frac{8 \ln \left(1+B_{M}\right)}{\rho_{F}}
$$

The $D^{2}$-law states that the square of droplet diameter is a linear function of time where the evaporation constant apparently represents the slope of the equation. The larger the $\lambda_{s t}$, the shorter the time it takes for the droplet to vaporize completely.

\section{Heat Transfer Number}

Consider conductive and convective heat fluxes across a thin shell surrounding the evaporating droplet, the heat transfer number is defined as the ratio of enthalpy available in the surrounding gas to the energy required to vaporize the fuel:

$$
B_{T}=\frac{c_{p, g}\left(T_{\infty}-T_{s}\right)}{L+c_{p, \text { drop }}\left(T_{s}-T_{d r o p}\right)}
$$

where $L$ is the latent heat of fuel vaporization corresponding to fuel surface temperature $[k J / k g]$.

For simplicity, one can neglect the energy required to raise the droplet temperature to the surface temperature. Then, equation $(\mathrm{K} .32$ becomes:

$$
B_{T}=\frac{c_{p, g}\left(T_{\infty}-T_{s}\right)}{L}
$$

When heat transfer dominates the evaporation process, the rate of evaporation of a fuel droplet at the surface is then described by:

$$
\dot{m}_{F, s}^{\prime \prime}=2 \pi D\left(\frac{k}{c_{p}}\right)_{A} \ln \left(1+B_{T}\right)
$$




\section{$B_{T}$ versus $B_{M}$}

Estimation of rate of fuel evaporation using equation $(\overline{\mathrm{K} .34})$ is only good for steady-state conditions. Nevertheless, equation (K.23) applies under all conditions, including the heat-up process of droplet [ref???].

However, under steady state conditions, $B_{M}=B_{T}=B$ and equation (K.23) and (K.34) are identical. Therefore, droplet evaporation rate can be written as:

$$
\dot{m}_{F, s}^{\prime \prime}=2 \pi D\left(\frac{k}{c_{p}}\right)_{A} \ln (1+B)
$$

\section{K.3.2 Heat-up Process}

According to Chin [6], serious error may be incurred in the calculation of fuel evaporation rate and droplet lifetime if the transient heat-up process is neglected. In fact, for many fuels at high ambient pressure and temperature, the transient heat-up process constitutes a significant portion of the droplet evaporation time.

At the steady-state period, the heat used in vaporizing the fuel is given by:

$$
Q_{s s}=\dot{m}_{F} L
$$

Substituting equation (K.15) into (K.36) yields

$$
Q_{s s}=2 \pi D\left(\frac{k}{c_{p}}\right)_{A} \ln \left(1+B_{M}\right) L
$$

Including the heating process, the actual heat transfer is estimated as

$$
Q_{s s}=2 \pi D\left(T_{\infty}-T_{s}\right)_{A} \frac{\ln \left(1+B_{M}\right)}{B_{M}}
$$

Then, the rate of change of the droplet surface temperature is given by 


$$
\frac{d T_{s}}{d t}=\frac{Q_{a c t}-Q_{s s}}{c_{p, F} m_{d r o p}}
$$

Substituting equation (K.37) and $(\mathrm{K} .38)$ into $(\mathrm{K} .39)$ and rearranging gives

$$
\frac{d T_{s}}{d t}=\frac{\dot{m}_{F} L}{c_{p, F} m_{d r o p}}\left(\frac{B_{T}}{B_{M}}-1\right)
$$

where

$$
m_{\text {drop }}=\frac{\pi}{6} \rho_{F} D^{3}
$$

Note that

$$
\dot{m}_{F}=\frac{d}{d t}\left(\frac{\pi}{6} \rho_{F} D^{3}\right)
$$

Equating equation $(\mathrm{K} .15)$ and $(\mathrm{K} .42)$ and rearranging gives the rate of change of droplet size

$$
\frac{d D}{d t}=\frac{4 \ln \left(1+B_{M}\right)}{\rho_{F} D}\left(\frac{k}{c_{p}}\right)_{A}
$$

\section{K.3.3 Droplet Lifetime}

Since the rate of chemical reactions in many practical combustion systems are so high, the burning rate is mainly controlled by the fuel evaporation process. Therefore, droplet lifetime is important in such situations because it determines the residence time needed to ensure completion of combustion.

Assume the final droplet diameter, $D_{0}$, equal to zero and rearranging the $D^{2}$-law, the steady state droplet lifetime is readily obtained by: 


$$
t_{s t}=\frac{D_{0}^{2}}{\lambda_{s t}}
$$

\section{K.3.4 Convective Effect}

All derivations shown above are only good for droplet at stationary condition. In addition, it is known that convection may enhance both mass and heat transfer during the evaporation process. Moreover, to include the convective effect into the evaporation rate equation is straightforward. Eq. (K.25) is then modified by replacing the coefficient with the Nusselt number correlation [16]:

$$
\dot{m}_{F}=N u \pi D\left(\frac{k}{c_{p}}\right)_{A} \ln \left(1+B_{M}\right)
$$

where

$$
\begin{gathered}
N u=2+0.6 \operatorname{Re}_{D}^{0.5} \operatorname{Pr}_{A}^{0.33} \\
R e_{d}=\frac{\rho_{A} \cdot U_{r} \cdot D}{\mu_{A}}
\end{gathered}
$$

\section{K.4 Numerical Method}

Six ordinary differential equations are to be solved for the six dependent variables $x, z, u_{d}, w_{d}, D$, and $T_{s}$. The droplet trajectory is defined by the set of $x$ and $z$ values. A $4^{\text {th }}$-Order Runge-Kutta explicit method [38] was used to solve these equations. The Runge-Kutta explicit method is an ideal numerical scheme for solving ordinary differential equations using Excel software. It is a self-starting method with good stability characteristics. The time step-size can be changed as desired without any complications for higher-order schemes.

There are totally six sets of coupled equations, namely the time rate change of $x, z, u_{d}, w_{d}, D$, and $T_{s}$, along with their solutions, as shown below: (subscript 
$n$ stands for the $n^{\text {th }}$ time step.)

[1]

$$
\begin{gathered}
\frac{d x}{d t}=u_{d}=f_{1}\left(u_{d}\right) \\
x_{n+1}=x_{n}+\frac{1}{6}\left(k_{1}+2 k_{2}+2 k_{3}+k_{4}\right)
\end{gathered}
$$

where

$$
\begin{aligned}
& k_{1}=\Delta t \cdot f_{1}\left(u_{d, n}\right) \\
& k_{2}=\Delta t \cdot f_{1}\left(u_{d, n}+\frac{l_{1}}{2}\right) \\
& k_{3}=\Delta t \cdot f_{1}\left(u_{d, n}+\frac{l_{2}}{2}\right) \\
& k_{4}=\Delta t \cdot f_{1}\left(u_{d, n}+l_{3}\right)
\end{aligned}
$$

$[2]$

$$
\begin{gathered}
\frac{d z}{d t}=w_{d}=f_{2}\left(w_{d}\right) \\
z_{n+1}=z_{n}+\frac{1}{6}\left(k z_{1}+2 k z_{2}+2 k z_{3}+k z_{4}\right)
\end{gathered}
$$


where

$$
\begin{aligned}
& k z_{1}=\Delta t \cdot f_{2}\left(w_{d, n}\right) \\
& k z_{2}=\Delta t \cdot f_{2}\left(w_{d, n}+\frac{l z_{1}}{2}\right) \\
& k z_{3}=\Delta t \cdot f_{2}\left(w_{d, n}+\frac{l z_{2}}{2}\right) \\
& k z_{4}=\Delta t \cdot f_{2}\left(w_{d, n}+l z_{3}\right)
\end{aligned}
$$

[3]

$$
\begin{gathered}
\frac{d u_{d}}{d t}=\frac{\left[-\frac{1}{2} \rho_{A}\left(u_{d}-u_{A}\right)\left|\vec{U}_{R}\right| A_{d} C_{D}\right]}{\rho_{d} V_{d}}=f_{3}\left(u_{d}, w_{d}, D, T_{s}\right) \\
u_{d, n+1}=u_{d, n}+\frac{1}{6}\left(l_{1}+2 l_{2}+2 l_{3}+l_{4}\right)
\end{gathered}
$$

where

$$
\begin{aligned}
& l_{1}=\Delta t \cdot f_{3}\left(u_{d, n}, w_{d, n}, D_{n}, T_{s, n}\right) \\
& l_{2}=\Delta t \cdot f_{3}\left(u_{d, n}+\frac{l_{1}}{2}, w_{d, n}+\frac{l z_{1}}{2}, D_{n}+\frac{m d_{1}}{2}, T_{s, n}+\frac{m t_{1}}{2}\right) \\
& l_{3}=\Delta t \cdot f_{3}\left(u_{d, n}+\frac{l_{2}}{2}, w_{d, n}+\frac{l z_{2}}{2}, D_{n}+\frac{m d_{2}}{2}, T_{s, n}+\frac{m t_{2}}{2}\right) \\
& l_{4}=\Delta t \cdot f_{3}\left(u_{d, n}+l_{3}, w_{d, n}+l z_{3}, D_{n}+m d_{3}, T_{s, n}+m t_{3}\right)
\end{aligned}
$$

[4]

$$
\begin{gathered}
\frac{d w_{d}}{d t}=\frac{\left[-\frac{1}{2} \rho_{A}\left(w_{d}-w_{A}\right)\left|\vec{U}_{R}\right| A_{d} C_{D}+\left(\rho_{A}-\rho_{d}\right) V_{d} g\right]}{\rho_{d} V_{d}}=f_{4}\left(u_{d}, w_{d}, D, T_{s}\right) \\
w_{d, n+1}=w_{d, n}+\frac{1}{6}\left(l z_{1}+2 l z_{2}+2 l z_{3}+l z_{4}\right)
\end{gathered}
$$


where

$$
\begin{aligned}
& l z_{1}=\Delta t \cdot f_{4}\left(u_{d, n}, w_{d, n}, D_{n}, T_{s, n}\right) \\
& l z_{2}=\Delta t \cdot f_{4}\left(u_{d, n}+\frac{l_{1}}{2}, w_{d, n}+\frac{l z_{1}}{2}, D_{n}+\frac{m d_{1}}{2}, T_{s, n}+\frac{m t_{1}}{2}\right) \\
& l z_{3}=\Delta t \cdot f_{4}\left(u_{d, n}+\frac{l_{2}}{2}, w_{d, n}+\frac{l z_{2}}{2}, D_{n}+\frac{m d_{2}}{2}, T_{s, n}+\frac{m t_{2}}{2}\right) \\
& l z_{4}=\Delta t \cdot f_{4}\left(u_{d, n}+l_{3}, w_{d, n}+l z_{3}, D_{n}+m d_{3}, T_{s, n}+m t_{3}\right)
\end{aligned}
$$

[5]

$$
\begin{gathered}
\frac{d D}{d t}=-\frac{\lambda}{2 D}=f_{5}\left(D, T_{s}\right) \\
D_{n+1}=D_{n}+\frac{1}{6}\left(m d_{1}+2 m d_{2}+2 m d_{3}+m d_{4}\right)
\end{gathered}
$$

where

$$
\begin{aligned}
& m d_{1}=\Delta t \cdot f_{5}\left(D_{n}, T_{s, n}\right) \\
& m d_{2}=\Delta t \cdot f_{5}\left(D_{n}+\frac{m d_{1}}{2}, T_{s, n}+\frac{m t_{1}}{2}\right) \\
& m d_{3}=\Delta t \cdot f_{5}\left(D_{n}+\frac{m d_{2}}{2}, T_{s, n}+\frac{m t_{2}}{2}\right) \\
& m d_{4}=\Delta t \cdot f_{5}\left(D_{n}+m d_{3}, T_{s, n}+m t_{3}\right)
\end{aligned}
$$

[6]

$$
\begin{gathered}
\frac{d T_{s}}{d t}=\frac{\dot{m}_{F} L}{c_{p, F} m_{d r o p}}\left(\frac{B_{T}}{B_{M}}-1\right)=f_{6}\left(u_{d}, w_{d}, D, T_{s}\right) \\
T_{s, n+1}=T_{s, n}+\frac{1}{6}\left(m t_{1}+2 m t_{2}+2 m t_{3}+m t_{4}\right)
\end{gathered}
$$


where

$$
\begin{aligned}
& m t_{1}=\Delta t \cdot f_{6}\left(u_{d, n}, w_{d, n}, D_{n}, T_{s, n}\right) \\
& m t_{2}=\Delta t \cdot f_{6}\left(u_{d, n}+\frac{l_{1}}{2}, w_{d, n}+\frac{l z_{1}}{2}, D_{n}+\frac{m d_{1}}{2}, T_{s, n}+\frac{m t_{1}}{2}\right) \\
& m t_{3}=\Delta t \cdot f_{6}\left(u_{d, n}+\frac{l_{2}}{2}, w_{d, n}+\frac{l z_{2}}{2}, D_{n}+\frac{m d_{2}}{2}, T_{s, n}+\frac{m t_{2}}{2}\right) \\
& m t_{4}=\Delta t \cdot f_{6}\left(u_{d, n}+l_{3}, w_{d, n}+l z_{3}, D_{n}+m d_{3}, T_{s, n}+m t_{3}\right)
\end{aligned}
$$




\section{Bibliography}

[1] Rosenberg, R., "All things to operators," Aerospace Engineeing, SAE International, Warrendale PA, Nov/Dec 2005.

[2] Andriani, R., Gamma, F., Ghezzi, U., and Infante, E., "Design Proposals For Constant Temperature Turbine Engine for Propulsion System," AIAA2001-3750, 37th AIAA/ASME/SAE/ASEE Joint Propulsion Conference and Exhibit, Salt Lake City, UT, July, 2001.

[3] Çengel, Y. A., and Cimbala, J. M., Fluid Mechanics: Fundamentals and Applications, McGraw Hill Inc., NY 2006, pp. 615-616.

[4] Chen, G., Hoffman, M. A., and Davis, R. L., "Gas-Turbine Performance Improvements Through the Use of Multiple Turbine InterStage Burners," Journal of Propulsion and Power, Vol. 20, No. 5, September-October 2004.

[5] Chin, J. S., and Lefebvre, A. H., "Steady-state Evaporation Characteristics of Hydrocarbon Fuel Drops", AIAA Journal, Vol. 21, No.10, pp. 1437-1443, 1983.

[6] Chin, J. S., and Lefebvre, A. H., "The role of the Heat-up Period in Fuel Drop Evaporation", Int. J. Turbo Jet Engines, Vol. 2, pp. 315-325, 1985.

[7] Chiu, Y.-T, "A Performance Study of a Super-cruise Engine with Isothermal Combustion inside the Turbine", Ph.D dissertation, Virginia Polytechnic Institute and State University, Blacksburg, VA, 2004.

[8] Crane, R. I., "A Critical Analysis of The Thermodynamic Advantages of Reheat in Gas Turbines," Proceeding of the Institute of Mechanical Engineers, Part A, Vol. 212, 1998.

[9] El-Masri M. A., "GASCAN - An Interactive Code for Thermal Analysis of Gas Turbine Systems," Journal of Engineering for Gas Turbines and Power, Vol. 110, April 1988.

[10] "Gas Turbine Engine Performance Station Identification and Nomenclature," Aerospace Recommended Practice (ARP) 755A, Society of Automotive Engineers, Warrendale, PA, 1974. 
[11] Glassman A. J., "Computer Program for Thermodynamic Analysis of OpenCycle Multishaft Power System with Multiple Reheat and Intercooler," NASA TN D-7589, March, 1974.

[12] Hanson, R. K., and Salimian, S., "Survey of Rate Constants in the N/H/O Systems," Chapter 6 in Combustion Chemistry (W. C. Gardiner, Jr., ed.), Springer-Verlag, New York, pp. 361-421, 1984.

[13] Hendricks, R. C., Liu, N.-S., Gallagher, J. R., Ryder, R. C., Brankovic, A., and Hendricks, J. A., "Modeling of a Sequential Two-Stage Combustor", NASA/TM-2005-212631, March 2005.

[14] Horlock, J. H., Watson, D. T., Jones, T. V., "Limitations on Gas Turbine Performance Imposed by Large Turbine Cooling Flow," Journal of Engineering for Gas Turbines and Power, Vol. 123, pp. 487-494, July 2001.

[15] Hünecke, Klaus, Jet Engine: Fundamentals of Theory, Design and Operation, Airlife Publishing Ltd, Shrewsbury, England, 1997

[16] Incropera, F. P., and DeWitt D. P., Fundamentals of Heat and Mass Transfer, 5th Edition, John Wiley \& Sons, New York, 2002.

[17] "Integrated High Performance Turbine Engine Technology (IHPTET)", Air Force Research Laboratory - Propulsion Directorate (AFRL/PR), http://www.pr.afrl.af.mil/divisions/prt/ihptet/ihptet.html

[18] "JANAF Thermochemical Tables", Second edition, The Dow Chemical Co., Midland, Michigan, 1971.

[19] Kesser, K. F., Hoffman, M. A., Baughn, J. W., "Analysis of a Basic Chemically Recuperated Gas Turbine Power Plant," Journal of Engineering for Gas Turbines and Power, Vol. 116, April 1994.

[20] Amsdem, A. A., "KIVA-3: A KIVA Program with Block-Structured Mesh for Complex Geometries", LA-12503-MS, 1993.

[21] Lefebvre, A. H., Gas Turbine Combustion, $1^{\text {st }}$ ed., Hemisphere Publishing Co., 1983.

[22] Lefebvre, A. H., "Fuel Effects on Gas Turbine Combustion-Liner Temperature, Pattern Factor, and Pollutant Emissions", Journal of Aircraft, Vol. 21, No.11, pp. 887-898, 1984.

[23] Leong, M.Y., McDonell, V.G., and Samuelsen, G.S., "Mixing of an AirblastAtomized Fuel Spray Injected Into a Crossflow of Air", NASA/CR-2000210467, 2000.

[24] Lewis, G. D., "Centrifugal-Force Effects on Combustion" $14^{\text {th }}$ Symposium (International) on Combustion, The Combustion Institute, pp. 413-419, 1973. 
[25] Liew, K. H., Urip, E., Yang, S. L., and Siow, Y. K., "A Complete Parametric Cycle Analysis of a Turbofan with Interstage Turbine Burner", AIAA2003-0685, 41st AIAA Aerospace Sciences Meeting and Exhibit, Reno, NV, January, 2003.

[26] Liew, K. H., Urip, E., Yang, S. L., Mattingly, J. D., Marek, C. J., "Performance Cycle Analysis of a Two-spool Separate-exhaust Turbofan with Interstage Turbine Burner", AIAA-2004-3311, 40th AIAA/ASME/SAE/ASEE Joint Propulsion Conference and Exhibit, Fort Lauderdale, FL, July, 2004.

[27] Liew K. H., Urip E., Yang S. L., Marek C. J., "An Interactive Microsoft Excel program for Tracking A Single Evaporating Droplet in Crossflow", NASA/TM-2004-212910, July, 2004.

[28] Liew K. H., Urip E., Yang S. L., "Parametric Cycle Analysis of a Turbofan with Interstage Turbine Burner", Journal of Propulsion and Power, Vol. 21, No.3, May-June 2005.

[29] Liew, K. H., Urip, E., Yang, S. L., Siow, Y. K., and Marek, C. J., "A Parametric (On-Design) Cycle Analysis for a Separate-Exhaust Turbofan Engine with Interstage Turbine Burner", NASA/TM-2005-213658, July 2005.

[30] Liew, K. H., Urip, E., Yang, S. L., Mattingly, J. D., Marek, C. J., "Performance (Off-Design) Cycle Analysis for a Turbofan Engine with Interstage Turbine Burner", NASA/TM-2005-213659, July 2005.

[31] Liew, K. H., Urip, E., Yang, S. L., Mattingly, J. D., Marek, C. J., "Performance Cycle Analysis of a Two-spool Separate-exhaust Turbofan with Interstage Turbine Burner", Journal of Propulsion and Power, Vol. 22, No.2, pp. 411-416, March-April 2006.

[32] Liu, F. and Sirignano, W.A., "Turbojet and Turbofan Engine Performance Increases Through Turbine Burners," Journal of Propulsion and Power, Vol 17, No. 3, pp. 695-705, May-June 2001.

[33] Mattingly, J. D., Elements of Gas Turbine Propulsion, McGraw Hill Inc., New York, pp. 18-31, 114-123, 240-246, 256-299, 346-361, 392-405, 1996.

[34] Mattingly, J. D., Heiser, W. H., and Pratt, D. T., Aircraft Engine Design, $2^{\text {nd }}$ ed., AIAA Education Series, AIAA, 2002.

[35] Mawid, M. A., Park, T. W., Thornburg, H., Sekar, B., and Zelina, J., "Numerical Analysis of ITB concepts and for improved gas turbine engine performance", AIAA-2005-1162, January 2005.

[36] "Model Specification for Engines, Aircraft, Turbojet", Military Specification $M I L-E-5008 B$, Department of Defense, January 1959. 
[37] "1991 Recipients of the Charles Stark Draper Prize", National Academy of Engineering, http://www.nae.edu/NAE/awardscom.nsf/weblinks/NAEW4NHMNB?opendocument .

[38] Nakamura, S., Applied Numerical Method With Software, Prentice Hall, Englewood Cliffs, NJ, 1991.

[39] Oates, G. C., Aerothermodynamics of Gas Turbine and Rocket Propulsion, 2nd Edition, AIAA Education Series, AIAA, Washington, DC, pp. 253-255, 1988 .

[40] Olikara, C., and Borman, G. L., "A Computer Program for Calculating Properties of Equilibrium Combustion Products with Some Applications to I. C. Engines," SAE Paper 750468, 1975.

[41] Papamoschou, D., "Engine Cycle and Exhaust Configurations for Quiet Supersonic Propulsion", AIAA-2002-3917, 38th AIAA/ASME/SAE/ASEE Joint Propulsion Conference and Exhibit, Indianapolis, IN, July 2002.

[42] Rice, I. G., "The Combined Reheat Gas Turbine/Steam Turbine Cycle: Part 1 - A Critical Analysis of the Combined Reheat Gas Turbine/Steam Turbine Cycle", ASME Paper No. 79-GT-7, 1979.

[43] Rogero, J. M., "A Genetic Algorithms Based Optimization Tool for The Preliminary Design of Gas Turbine Combustor", Ph.D dissertation, Cranfield University, UK, 2002.

[44] Rutar, T., Horning, D. C., Lee, J. C. Y., and Malte, P. C., "NOx Dependency on Residence Time and Inlet Temperature for Lean-Premixed Combustion in Jet-Stirred Reactors," ASME Paper No. 98-GT-433, 1998.

[45] Saravanamuttoo, H. I. H., Rogers, G. F. C., and Cohen, H., Gas Turbine Theory, $5^{\text {th }}$ ed., Pearson Education Limited, Harlow, England, 2001.

[46] Siow, Y. K., and Yang, S. L., "Numerical Study And Design Of Interstage Turbine Burner", AIAA-2002-3727, 38th AIAA/ASME/SAE/ASEE Joint Propulsion Conference and Exhibit, Indianapolis IN, July 2002.

[47] Sirignano, W.A., Delplanque, J. P., and Liu, F., "Selected Challenges in Jet and Rocket Engine Combustion Research," AIAA-2002-3727, 33rd AIAA/ASME/SAE/ASEE Joint Propulsion Conference and Exhibit, Seattle WA, July 6-9 1997.

[48] Sirignano, W.A., and Liu, F., "Performance Increases for Gas-Turbine Engines Through Combustion Inside the Turbine," Journal of Propulsion and Power, Vol. 15, No. 1, pp 111-118, January-February 1999. 
[49] Sturgess, G. J., Zelina, J., Shouse, D. T., Roquemore W. M., "Emissions Reduction Technologies for Military Gas Turbine Engines', Journal of Propulsion and Power, Vol. 21, No.2, pp. 193-217, March-April 2005.

[50] Takeya, K., Oteki, Y., and Yasui, H., "Current Status of Advanced Reheat Gas Turbine AGTJ-100A(part 3):Experimental Results of Shop Tests", ASME Paper No. 84-GT-57, 1984.

[51] Turns, S. R., An Introduction to Combustion:Concepts and Applications, $2^{\text {nd }}$ ed., McGraw Hill, Singapore, 2000.

[52] Urip E., Yang S. L., Marek C. J., "An Interactive Microsoft Excel program for Tracking A Single Droplet in Crossflow Computation", NASA/TM-2002211710, August, 2002.

[53] Vogeler, K., "The Potential of Sequential Combustion For High Bypass Jet Engines", Proceedings of the International Gas Turbine 83 Aeroengine Congress 83 Exhibition, Stockholm, Sweden, June 1998; also ASME 98-GT311, 1998.

[54] Walsh, P. P., and Fletcher, P., Gas Turbine Performance, 2nd ed., Blackwell Publishing and ASME, Fairfield, NJ, pp. 227 and 282, 2004.

[55] Young, J. B., and Wilcock, R. C., "Modeling the Air-Cooled Gas Turbine: Part 1 - General Thermodynamics," Journal of Turbomachinery, Vol. 124, No. 2, pp. 207-213, April 2002.

[56] Zelina, J., Sturgess, G. J., and Shouse, D. T., "The Behavior of UltraCompact Combustor Based on Centrifugally-enhanced Turbulent Burning Rates", AIAA-2004-3541, July 2004.

[57] Zucrow, M. J., Aircraft and Missile Propulsion: Volume II, Wiley, New York, pp. 52-53, 1964. 


\section{Vita}

The author, Ka Heng Liew, was born in Kuala Lumpur (KL), Malaysia in year 1979. He grew up in KL and graduated from SRJK(c) Chong Hwa elementary school located at Setapak, KL in 1991. Then, he attended Chong Hwa Independent High School at Jalan Ipoh, KL and left the school in 1997. In the same year, he joined the American Degree Program at Sepang Institute of Technology (SIT), Selangor state for $1 \frac{1}{2}$ years and was majoring in engineering science. Financially affected by the East Asian financial crisis in 1998, he postponed his plan to study abroad and decided to become a temporary teacher at his alma mater at Setapak for 6 months.

In 1999, he came to United States of America and continued his undergraduate study at Michigan Technological University, Houghton, MI. In the summer of 2000, he went to do his co-op assignment at Steelcase Inc., Grand Rapids, MI. He completed his Bachelor degree in Mechanical Engineering with summa cum laude in 2001.

With a strong interest in energy transformation and utilization, he decided to stay in Houghton and enrolled in a Doctor of Philosophy degree program specializing in energy-thermal-fluid area. He became a teaching assistant for a mechanical engineering laboratory. Then, in collaboration with NASA Glenn Research Center, he first co-developed a code to track evaporating droplet trajectory in crossflow inside a combustor with his colleague, Mr. Egel Urip. This work was awarded NASA Space Act Award in 2005. Later, he successfully developed two simulation codes to perform aerothermodynamic cycle analysis of a turbofan engine with an Interstage Turbine Burner, which is well-documented in this dissertation. All codes mentioned above were written in both Microsoft Visual Basic and FORTRAN programming languages. Besides, he also used 
a widely-used KIVA engine code to perform flow-field simulations inside an Advanced Subsonic Combustor Rig (ASCR) and Lean-Premixed Prevaporized (LPP) combustor.

After graduation in 2006, he will start working as an engineer in Engineering Applications Powertrain division at Robert Bosch Corporation, Farmington Hill, MI. 